\title{
On the Discovery of Semantically Meaningful SQL Constraints from Armstrong Samples: Foundations, Implementation, and Evaluation
}

\author{
by \\ Van Tran Bao Le
}

A thesis

submitted to the Victoria University of Wellington in fulfilment of the requirements for the degree of Doctor of Philosophy in Information Management.

Victoria University of Wellington 2014 



\begin{abstract}
A database is said to be $C$-Armstrong for a finite set $\Sigma$ of data dependencies in a class $C$ if the database satisfies all data dependencies in $\Sigma$ and violates all data dependencies in $C$ that are not implied by $\Sigma$. Therefore, Armstrong databases are concise, user-friendly representations of abstract data dependencies that can be used to judge, justify, convey, and test the understanding of database design choices. Indeed, an Armstrong database satisfies exactly those data dependencies that are considered meaningful by the current design choice $\Sigma$. Structural and computational properties of Armstrong databases have been deeply investigated in Codd's Turing Award winning relational model of data. Armstrong databases have been incorporated in approaches towards relational database design. They have also been found useful for the elicitation of requirements, the semantic sampling of existing databases, and the specification of schema mappings.

This research establishes a toolbox of Armstrong databases for SQL data. This is challenging as SQL data can contain null marker occurrences in columns declared NULL, and may contain duplicate rows. Thus, the existing theory of Armstrong databases only applies to idealized instances of SQL data, that is, instances without null marker occurrences and without duplicate rows. For the thesis, two popular interpretations of null markers are considered: the no information interpretation used in SQL, and the exists but unknown interpretation by Codd. Furthermore, the study is limited to the popular class $C$ of functional dependencies. However, the presence of duplicate rows means that the class of uniqueness constraints is no longer subsumed by the class of functional dependencies, in contrast to the relational model of data. As a first contribution a provably-correct algorithm
\end{abstract}


is developed that computes Armstrong databases for an arbitrarily given finite set of uniqueness constraints and functional dependencies. This contribution is based on axiomatic, algorithmic and logical characterizations of the associated implication problem that are also established in this thesis. While the problem to decide whether a given database is Armstrong for a given set of such constraints is precisely exponential, our algorithm computes an Armstrong database with a number of rows that is at most quadratic in the number of rows of a minimum-sized Armstrong database. As a second contribution the algorithms are implemented in the form of a design tool. Users of the tool can therefore inspect Armstrong databases to analyze their current design choice $\Sigma$. Intuitively, Armstrong databases are useful for the acquisition of semantically meaningful constraints, if the users can recognize the actual meaningfulness of constraints that they incorrectly perceived as meaningless before the inspection of an Armstrong database. As a final contribution, measures are introduced that formalize the term "useful" and it is shown by some detailed experiments that Armstrong tables, as computed by the tool, are indeed useful.

In summary, this research establishes a toolbox of Armstrong databases that can be applied by database designers to concisely visualize constraints on SQL data. Such support can lead to database designs that guarantee efficient data management in practice. 


\section{Acknowledgments}

My first acknowledgement is to Sebastian Link. You not only guided me during the development of this thesis, but also willingly showed me how to sharpen and publish research results and deal with critical comments. Thank you, Sebastian, for your patient guidance, enthusiastic encouragement, and excellent support that made it possible for me to complete this work. Your positive outlook and confidence in my capabilities inspired me, making the tough PhD journey become enjoyable. It has been an honor to be your PhD student, and I could not have imagined having a better supervisor for my PhD study.

Thanks also to Flavio Ferrarotti. Despite your busy schedule, you always made yourself available to clarify my doubts. Your guidance and advice served me well, and I owe you my heartfelt appreciation. I also thank David Mason. You welcomed me warmly and made the last two years of my $\mathrm{PhD}$ journey smooth through your supervision and timely assistance. My grateful thanks are also extended to Dan Dorner, Val Hooper, and Pak Yoong. Your open doors and ready support were much appreciated. Thanks to Usha Varatharaju, Jean Grant, and Tiso Ross. You helped me navigate bureaucratic pathways. My thanks also go to Dion Peszynski. Without you, the web application developed for this work could not have gone live.

Thanks to the many other helpful and generous people at Victoria University of Wellington and particularly the academic, administrative, and technical staff of the School of Information Management: Jocelyn Crane- 
field, Chern Li Liew, Gillian Oliver, Lalita Rajasingham, Mary Tate, Janet Toland, Brenda Chawner, Alastair Smith, Christine King, and Cristina Sebold. It has been a great privilege to spend four years in the School of Information Management (SIM) at Victoria University of Wellington, and its members will always remain dear to me.

I would especially like to acknowledge Palve Mogin and Hui Ma in the School of Engineering and Computer Science at Victoria University of Wellington, and Nguyen Thi Thanh Tam in the Computer Science Department at Lotus University of Vietnam. You were there to kindly assist me during the data gathering process. Thanks to all the computer science students for participating in my experiments. Without them, I simply could not have conducted empirical studies and completed this thesis.

Finally, I wish to thank my parents, Le Duy Quang and Tran Thi Phuong Mai, who have never lost their belief in me and helped me go the distance. Thanks to my husband, Nguyen Trong Duc, for your unconditional support by being willing to share the ups and downs of the $\mathrm{PhD}$ process despite the massive challenges you had to face when making a big move to New Zealand to support me. Thanks also to my great friends in New Zealand and in Vietnam for your support and encouragement, for stepping in when things got tough, and making me take out time for a bit of fun every now and then. 


\section{Contents}

\begin{tabular}{lll}
\hline & Introduction & $\mathbf{1}$
\end{tabular}

1.1 Challenges with constraints . . . . . . . . . . . . . 1

1.2 State of database practice . . . . . . . . . . . . . . 2

1.3 State of database theory . . . . . . . . . . . . . 3

1.4 State of disparity . . . . . . . . . . . . . 3

1.5 Objective and approach of the thesis . . . . . . . . . . 4

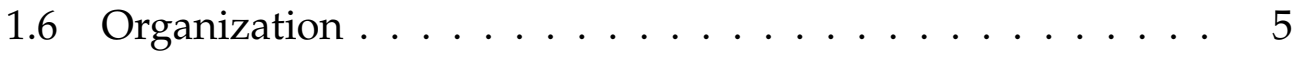

1.7 Publications . . . . . . . . . . . . . 6

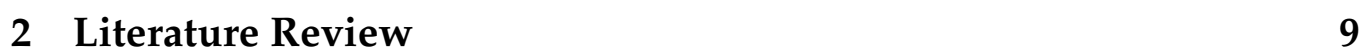

2.1 Data Dependencies over Relations . . . . . . . . . . . . . . 9

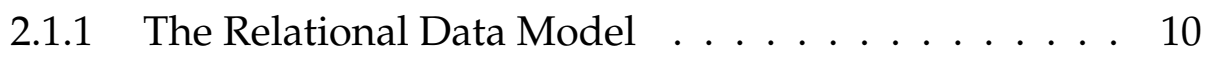

2.1 .2 Data Dependencies . . . . . . . . . . . . . . 11

2.1 .3 The Class of Keys . . . . . . . . . . . . . . . . . . . . 14

2.1 .4 The Class of Functional Dependencies . . . . . . . . . 15

2.2 Armstrong Relations . . . . . . . . . . . . . . . . . . . 21

2.2.1 General Definition of an Armstrong Relation . . . . . 23

2.2.2 Armstrong Relations for Functional Dependencies. . 24

2.2.3 Evidence For the Usefulness of Armstrong Relations 31

2.2.4 Constraint Acquisition by Sample Data and Natural Language Processing . . . . . . . . . . . . . 32

2.3 Partial Information in Databases . . . . . . . . . . . . 33

2.3 .1 No Information . . . . . . . . . . . . . . . . . . 34 
2.3 .2 Value Unknown at Present . . . . . . . . . . . . . 35

2.4 Data Dependencies over Partial Relations . . . . . . . . . 36

2.4.1 Functional Dependencies over SQL Relations. . . . . 36

2.4 .2 FDs and NOT NULL constraints over SQL Relations 39

2.4 .3 FDs over Codd Relations . . . . . . . . . . . . . . 42

2.4 .4 Uniqueness Constraints and Keys . . . . . . . . . 44

2.5 SQL Armstrong Tables $\ldots \ldots \ldots$

2.5 .1 NFDs Do Not Enjoy SQL Armstrong Tables. . . . . . 50

2.5 .2 Structural Properties of SQL Armstrong Tables . . . . 50

2.5 .3 Computational Properties . . . . . . . . . . . . 54

2.5 .4 Further Remarks . . . . . . . . . . . . . . . . . . . 59

2.6 Summary and Research Gap. . . . . . . . . . . . . . . . 60

2.6 .1 Summary . . . . . . . . . . . . . . 60

2.6 .2 Research gap $\ldots \ldots \ldots \ldots$. . . . . . . . . . 61

2.6 .3 Objectives $\ldots \ldots \ldots \ldots \ldots$

3 Foundations - Part I $\quad 65$

3.1 Preliminary Definitions. . . . . . . . . . . . . . . . 66

3.2 Axiomatic Characterization . . . . . . . . . . . . 71

3.3 Algorithmic Characterization . . . . . . . . . . . . . . 78

3.4 Logical Characterization . . . . . . . . . . . . . . . . 83

$3.4 .1 \quad \mathcal{S}$-3 Logics $\ldots \ldots \ldots \ldots$. . . . . . . . . . . . 84

$3.4 .2 \quad$ Equivalence of Implication Problems $\ldots \ldots$. . . . 85

3.5 Further Comments . . . . . . . . . . . . . . . . . . 90

3.6 Summary $\ldots \ldots \ldots \ldots \ldots$

\begin{tabular}{lll}
\hline & Foundations - Part II & 93
\end{tabular}

4.1 Two Motivating Examples . . . . . . . . . . . . . . . . . . . 94

4.2 Structural Properties . . . . . . . . . . . . . . . . . . . . . 95

4.3 Computational Properties . . . . . . . . . . . . . . . . 102

$4.3 .1 \quad$ Computation of Maximal Sets. . . . . . . . . . . . . 102

4.3 .2 Computation of Duplicate Sets . . . . . . . . . 106 
4.3 .3 Computation of Armstrong Tables . . . . . . . . . . . 109

4.4 Complexity Considerations . . . . . . . . . . . . . . . 114

4.4.1 Worst-case Complexity to Find Armstrong Tables . . 114

4.4 .2 Minimum-sized Armstrong Tables . . . . . . . . . . 116

4.4 .3 The Size of Representations . . . . . . . . . . . . . . 117

4.5 Further Remarks . . . . . . . . . . . . . . . . . . . . . 120

4.6 Summary . . . . . . . . . . . . . . . . . . . 122

5 Implementation: SQL-Sampler 123

5.1 Introduction to SQL-Sampler . . . . . . . . . . . . . . . . . . 124

5.2 System Requirements . . . . . . . . . . . . . . . . . . . . 127

5.3 Design . . . . . . . . . . . . . . . . . . . . . 127

5.3 .1 DEFINE CONTEXT module . . . . . . . . . . . . 127

5.3 .2 INPUT DATA module . . . . . . . . . . . . . . . 130

5.3 .3 COMPUTE module. . . . . . . . . . . . . . . . . . 130

5.3 .4 OUTPUT DATA module . . . . . . . . . . . . . . 133

5.3 .5 ARMSTRONGTABLE database . . . . . . . . . . . 133

5.4 Implementation Details . . . . . . . . . . . . . . . 136

5.4 .1 Context variable . . . . . . . . . . . . . 137

5.4 .2 DATA INPUT functions $\ldots \ldots \ldots$. . . . . . . 137

5.4 .3 COMPUTATION functions . . . . . . . . . . . . 138

5.4 .4 DATA OUTPUT functions . . . . . . . . . . . . . . 143

5.5 Use Case Example . . . . . . . . . . . . . . . . . . . . . . . . 145

5.5 .1 The Use Case . . . . . . . . . . . . . . . . . . . 145

5.5 .2 Context . . . . . . . . . . . . . . . 145

5.5 .3 Input Data . . . . . . . . . . . . . . . . 145

5.5 .4 Computing Armstrong Table . . . . . . . . . . . 146

5.5 .5 Output . . . . . . . . . . . . . . . 146

5.6 Summary . . . . . . . . . . . . . . . . . . . . 149

6 Evaluation: Experiment Design 151

$6.1 \quad$ The overall process of the experiment . . . . . . . . . . . 152 
6.2 Participants . . . . . . . . . . . . . . . . 154

6.3 Application domain and the target set . . . . . . . . . . 155

6.4 Quality measures . . . . . . . . . . . . . . . . . . 156

6.4 .1 Preliminary notation . . . . . . . . . . . 156

6.4 .2 Soundness . . . . . . . . . . . . . . . 158

6.4 .3 Completeness . . . . . . . . . . . . . . 159

6.4 .4 Proximity . . . . . . . . . . . . . . 159

6.4 .5 Gain . . . . . . . . . . . . . . 160

6.4 .6 Actual and possible gain . . . . . . . . . . . . 161

6.5 Example . . . . . . . . . . . . . . . . . . . 162

6.5 .1 Data gathering . . . . . . . . . . . . 162

6.5 .2 Data analysis . . . . . . . . . . . . 163

6.6 Further considerations and future research . . . . . . . . 170

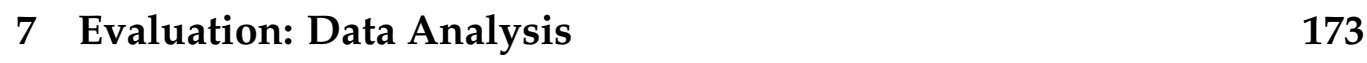

7.1 Quantitative data analysis . . . . . . . . . . . . . 173

7.1 .1 Quantitative analysis for soundness . . . . . . . . . 174

7.1.2 Quantitative analysis for completeness . . . . . . . 180

7.1.3 Quantitative analysis for proximity . . . . . . . 186

7.1 .4 Quantitative analysis for groups . . . . . . . . . . 191

7.2 Qualitative data analysis . . . . . . . . . . . . . . 195

7.2.1 Qualitative analysis for soundness . . . . . . . . . 196

7.2.2 Qualitative analysis for completeness . . . . . . . . 202

7.2.3 Qualitative analysis for proximity . . . . . . . . . 208

7.2.4 Qualitative analysis by undiscovered constraints. . . 211

7.2.5 Qualitative analysis per groups . . . . . . . . . 213

7.3 Summary . . . . . . . . . . . . . . . . . . 216

8 Conclusion and Future Work 217

8.1 Conclusion . . . . . . . . . . . . . . . . . . . . 217

8.2 Future Work . . . . . . . . . . . . . . . . . . . . . . . . . . . 219 
CONTENTS ix

\begin{tabular}{|l|l}
\hline Appendices & 223
\end{tabular}

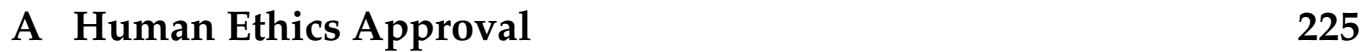

B Experiment Information and Consent Forms 233 


\section{Chapter 1}

\section{Introduction}

Constraints largely define the world we live in. For examples, people can only be at one place at a time, every species of echidna belongs to the class of mammals, and the National Health Index number (NHI number) uniquely identifies every person who uses health and disability support services in New Zealand. Database systems manage data about an application domain in our world. Therefore, effective data management and transforming data into value require databases to satisfy the constraints that define the application domain [1, 41, 87].

\subsection{Challenges with constraints}

Constraints are fundamental for database modeling and design [97, 105. [123]. They are required for essential data management services such as updates [46,76, 128], queries [116], security [19], sampling [36], data cleaning [51], exchange [98] and integration [23]. It is a major challenge to identify classes of constraints that are precious. That is, constraints in these classes are able to express important properties about application domains and database management systems can maintain them efficiently. Efficient maintenance entails that the implication problem, associated with this class of constraints, can be decided efficiently [49,127]. The $C$-implication 
problem is to decide if for any given set $\Sigma \cup\{\varphi\}$ of constraints in $C, \Sigma$ implies $\varphi$, that is, every database that satisfies all the constraints in $\Sigma$ also satisfies $\varphi$. For very expressive classes the implication problem becomes infeasible. A prime example is the class of first-order formulae [18]. Examples of precious classes of constraints include uniqueness constraints and so-called functional dependencies [1, 41, 87, 94, 97, 122, 123, 125]. Given that precious classes of constraints have been identified, a second challenge is to discover the constraints in this class which are semantically meaningful for the application domain of interest. Failure to discover a semantically meaningful constraint entails that the database management system permits database instances that are not representations of the real world. While this thesis is mainly concerned with addressing the second challenge, the first challenge must be addressed beforehand.

\subsection{State of database practice}

Recently, the database industry has been worth more than 32 billion US dollars, and was still growing in the double digits [100]. Database systems can be purchased from vendors such as Oracle, IBM and Microsoft, and are available in the form of open-source software including MySQL, PostgreSQL and Ingres. Most data are managed by database systems that implement the ISO and ANSI industry standard for defining and querying data, that is, the Structured Query Language (SQL) [33]. SQL was developed by IBM [26] to implement Edgar Codd's relational model of data [28]. After more than 40 years in use, SQL-based database systems still dominate the market today and influence new paradigms as the field evolves [4]. Web models, such as XML and RDF, are applied primarily to roll-out, exchange and integrate data that are commonly SQL-based [41]. Many websites, such as Facebook, and distributed applications, such as ecommerce, require high scalability. However, their core data stores and services remain SQL-based [108]. While SQL covers Codd's relational 
model of data, additional features are meant to ease data processing [33]. SQL data are laid out in tables. Tables can contain i) duplicate rows to avoid expensive duplicate removal, and ii) null markers to accommodate partial information in columns declared NULL. Relations are tables with no duplicate rows and no null marker occurrences.

\subsection{State of database theory}

Codd introduced the relational model of data in his seminal paper in 1970 [28]. His overall achievement was to transform data management into a science. In 1981 Codd received the Turing Award "for his fundamental and continuing contributions to the theory and practice of database management systems". Mainstream research has addressed the above challenges in the relational model of data [49]. Approximately one hundred different classes of constraints on relations have been investigated [122], but few of them are motivated by practice. Research has shown that precious classes of constraints can enable quality data management in theory $[1,87,94,97,125]$. Some researchers have examined the effect of null markers on the implication problem for some classes of constraints $[8,72,87,89]$. Surprisingly, there is very little work in the literature that analyzes constraints on general SQL tables. Instead, the community attempts to understand constraints on Web data $[5,6,17,67,68,80,129]$. An investigation of constraints on SQL data would not just address the dominant data format in practice, but also contribute to our understanding of more sophisticated formats, such as Web data.

\subsection{State of disparity}

We observe: 1) In practice, SQL data is the premier data format, and 2) In theory, constraints on SQL have not received sufficient attention. Moreover, $C$-implication problems in the presence of partial information are 
already more intricate than those over relations [8, 69]. Thus, the current foundation for constraints applies to only very special instances of SQL data. This distinct disparity between theory and practice restrains the quality of data management severely.

\subsection{Objective and approach of the thesis}

The main objective of the thesis is to address the above challenges with constraints in SQL databases. More precisely, the underlying research question asks:

How can database designers discover real-world database constraints that are meaningful for the given application domain?

In particular, the goal of the thesis is to provide tool support to discover semantically meaningful SQL constraints effectively.

The concept of Armstrong databases [47, 48, 96] will be investigated in the context of SQL data in order to address this goal. Informally, Armstrong databases are sample databases that perfectly represent a given collection of database constraints. So far, the concept of Armstrong databases has mainly been investigated for relations only, that is, for idealized instances of SQL data. In this special case, structural and computational properties of Armstrong databases have been studied. Based on these properties, prototypes have been built to compute Armstrong databases. Finally, these prototypes have been used to establish empirical evidence for the usefulness of Armstrong databases in the discovery of semantically meaningful constraints.

The goal of the thesis is to develop a toolbox of Armstrong databases for the precious classes of uniqueness constraints and functional dependencies over SQL data. As these are the most popular classes of constraints and SQL is the dominant data format in practice, such a contribution would have an impact on database theory, practice and educa- 
tion. From a theoretical perspective, the contribution would provide a better understanding of SQL constraints, extending our knowledge from the idealized special case of strictly relational constraints. From a practical perspective, database designers can apply the toolbox to discover a more complete set of semantically meaningful SQL constraints. Ultimately, this would lead to better database designs and improved data processing capabilities. From an educational point of view, the measures for assessing the usefulness of Armstrong databases can be used to mark certain nonmultiple choice questions in database course assignments and exams. The computation of the Armstrong databases itself can be used as a means to provide automated feedback to students.

\subsection{Organization}

Chapter 2 contains a review of the literature. In particular, the terms used informally in this introductory chapter will be defined formally. The chapter will discuss previous work on data dependencies and Armstrong databases over relations with a particular focus on uniqueness constraints and functional dependencies. The literature review will continue with a discussion of two popular approaches to encompass partial information in databases, as well as previous work on the implication problem and Armstrong databases for uniqueness constraints and functional dependencies in this context. The chapter ends with a summary, states a research gap, and the objectives of the thesis.

Chapter 3 and Chapter 4 are concerned with foundations. In Chapter 3 specifically, the implication problem for a combined class of uniqueness constraints, NOT NULL constraints and functional dependencies is characterized axiomatically, algorithmically, and logically, following Codd's original proposal to interpret null markers in databases as value unknown at present. In Chapter 4 , structural and computational properties of Armstrong tables for this class are established. 
Chapter 5 presents the actual toolbox, called SQL-Sampler, developed in this research. SQL-Sampler provides Web-based and desktop-based graphical user interfaces that allow its users to compute Armstrong tables for various classes of SQL constraints, including those from the recent literature as well as the class investigated in the previous two chapters. Chapter 5 contains a description of the system requirements, the design of SQL-Sampler, implementation details, as well as a use case example with screenshots.

Chapter 6 and Chapter 7 are concerned with the empirical evaluation of the toolbox, and more generally, the usefulness of Armstrong tables for the discovery of meaningful SQL constraints. Chapter 6 describes the design of the experiment and introduces several measures that formalize the notion of "usefulness". Chapter 7 contains the quantitative and qualitative analysis of the data gathered in the experiments. It shows that Armstrong tables, as produced by SQL-Sampler, are indeed useful for the discovery of actually meaningful SQL uniqueness constraints and functional dependencies that are incorrectly perceived as meaningless prior to the inspection of these Armstrong tables. It also shows that Armstrong tables are not useful for the discovery of actually meaningless SQL uniqueness constraints and functional dependencies that are incorrectly perceived as meaningful prior to the inspection of these Armstrong tables.

The conclusion of the thesis is presented in Chapter 8 which contains a summary of the main results as well as an outlook into possible future work.

\subsection{Publications}

Several of the results that will be presented in this thesis have been announced in international conferences and journals. These are:

- V. B. T. Le, S. Link, and F. Ferrarotti, "SQL-Sampler: A Tool to Visualize and Consolidate Domain Semantics by Perfect SQL Sample 
Data", in Proceedings of the 10th Asia-Pacific Conference on Conceptual Modeling (APCCM) (G. Grossmann and M. Saeki, eds.), vol. 154 of Conferences in Research and Practice in Information Technology, 10 pages, Australian Computer Society, 2014.

This paper received the Best Student Paper Award.

- V. B. T. Le, S. Link, and F. Ferrarotti, "Effective recognition and visualization of semantic requirements by perfect SQL samples", in Proceedings of the 32nd International Conference on Conceptual Modeling (ER) (W. Ng, V. Storey, and J. Trujillo, eds.), vol. 8217 of Lecture Notes in Computer Science, pp. 227-240, Springer, 2013.

This paper received the Best Student Paper Award.

- V. B. T. Le, S. Link, and M. Memari, "Schema- and data-driven discovery of SQL keys", Journal of Computing Science and Engineering, vol. 6, no. 3, pp. 193-206, 2012.

- V. B. T. Le, S. Link, and M. Memari, "Discovery of keys from SQL tables", in Proceedings of the 17th International Conference on Database Systems for Advanced Applications (DASFAA) (S. Goo Lee, Z. Peng, X. Zhou, Y.-S. Moon, R. Unland, and J. Yoo, eds.), vol. 7238 of Lecture Notes in Computer Science, pp. 48-62, Springer, 2012.

- F. Ferrarotti, S. Hartmann, V. B. T. Le, and S. Link, “Codd table representations under weak possible world semantics", in Proceedings of the 22nd International Conference on Database and Expert Systems Applications (DEXA), Part I (A. Hameurlain, S.W. Liddle, K.-D. Schewe, and X. Zhou, eds.), vol. 6860 of Lecture Notes in Computer Science, pp. 125-139, Springer, 2011. 


\section{Chapter 2}

\section{Literature Review}

This chapter provides a focused literature review of the existing work on some popular classes of constraints: keys and functional dependencies. The review is based on the two challenges with constraints, identified in Chapter 1. In Section 2.1 well-known results on the implication problem for the classes of keys and functional dependencies over pure relations are summarized. Armstrong relations for these two classes are discussed in Section 2.2. Section 2.3 briefly reviews two popular interpretations of null markers in partial relations: no information and value unknown at present. Subsequently, previous work on keys and functional dependencies over partial relations is discussed in Section 2.4. following both of these interpretations. Results on Armstrong tables for keys and functional dependencies over partial relations are given in Section 2.5. In Section 2.6, the literature review is summarized, existing gaps in the research literature are listed, and the objectives of the thesis are stated.

\subsection{Data Dependencies over Relations}

The purpose of this section is to summarize well-known results on the implication problem for the class of keys and functional dependencies in the relational model of data. Keys and functional dependencies form ar- 
guably the most popular class of data dependencies in database research and practice.

\subsubsection{The Relational Data Model}

The relational model of data [28], or simply the relational model, refers to a database model with at least the following three components:

- Structural component: a set of relations, each of which can be represented in the form of a table containing values.

- Integrity component: integrity constraints which identify the relations that are semantically meaningful in the given application domain.

- Manipulative component: languages to define data structures and semantics, and to perform updates and queries.

For the purpose of this thesis, only the structural and the integrity component are of interest. Excellent resources for a broader introduction to the relational model of data include [1, 87, 94].

It is essential to distinguish between the syntax and semantics in databases. On the syntactic level we have relation schemata. These model the properties that all entities of interest are described by. Formally, a relation schema is a finite non-empty set $R$ of elements, called attributes. Each attribute $A \in R$ denotes a property of each entity of interest. The possible values of an attribute $A$ are taken from the domain $\operatorname{dom}(A)$ of $A$.

On the semantical level we have relations. These are finite sets of tuples. Formally, a tuple $t$ over the relation schema $R$ is a function $t: R \rightarrow$ $\bigcup_{A \in R} \operatorname{dom}(A)$ that assigns to every attribute $A \in R$ a value $t(A) \in \operatorname{dom}(A)$. Relations over a relation schema are commonly illustrated in the form of tables. As an example consider a simple database about employees in Table 2.1 


\begin{tabular}{ccc}
\hline Emp & Dept & Mgr \\
\hline Dilbert & Information Systems & Gates \\
Alice & Information Systems & Gates \\
Wally & E-commerce & Jobs \\
\hline
\end{tabular}

Table 2.1: The relation $r_{\text {total }}$ over WORK

The relation in Table 2.1 is defined over the relation schema WORK. The latter consists of the attributes Emp, Dept and Mgr. Intuitively, tuples over the schema consist of an employee who works in a department which has a manager. As domains for the attributes one can choose suitably restricted sets of strings.

\subsubsection{Data Dependencies}

Data dependencies are semantically meaningful and syntactically restricted sentences of the predicate calculus that must be satisfied by any legal relation [122]. Their presence remedies some of the semantic poverty of relations, e.g., with pure relations one has trouble representing the fact that some relationships among attributes are one-to-one or one-to-many [1, 122].

In general, a dependency over a relation schema $R$ is a function $d$ that maps every relation $r$ over $R$ to a value $d(r) \in\{0,1\}$. If $d(r)=1$, we say that relation $r$ satisfies the dependency $d$. Otherwise, we say that $r$ does not satisfy $d$, or that $r$ violates $d$. We say that a relation $r$ satisfies a set $\Sigma$ of data dependencies, if $r$ satisfies every data dependency $\sigma \in \Sigma$. Throughout this thesis we will use the terms constraint and data dependency interchangeably. Strictly speaking, data dependencies form a subclass of integrity constraints [122], but the difference is not important for this thesis. In general, we will use the term that is most common in the literature, e.g. there will be uniqueness constraints and functional dependencies. 


\section{Semantic Implication}

Central to the study of data dependencies is their implication problem. The following subsections review principle concepts of data dependencies [1, 49, 87, 94, 122].

Suppose we fix a given class $\mathcal{C}$ of data dependencies. Then the implication problem for $\mathcal{C}$ is to decide whether for any given finite set $\Sigma \cup\{\varphi\}$ of data dependencies in $\mathcal{C}$ it is true that $\Sigma$ implies $\varphi$. Here, $\Sigma$ implies $\varphi$ if every relation that satisfies $\Sigma$ also satisfies $\varphi$. In other words, there is no relation $r$ that satisfies $\Sigma$ and violates $\varphi$. If $\Sigma$ implies $\varphi$, then we write $\Sigma \models \varphi$. Otherwise, we write $\Sigma \not \models \varphi$.

The set $\Sigma^{*}=\{\varphi \in \mathcal{C} \mid \Sigma \models \varphi\}$, that consists of all data dependencies in $\mathcal{C}$ implied by $\Sigma$, is called the semantic closure of $\Sigma$. The implication problem for the class $\mathcal{C}$ is to decide whether for an arbitrarily given relation schema $R$, and an arbitrarily given set $\Sigma \cup\{\varphi\}$ of data dependencies in $\mathcal{C}$ over $R$, $\Sigma \models \varphi$ holds.

For two sets $\Sigma_{1}$ and $\Sigma_{2}$ of data dependencies in $\mathcal{C}$, we call $\Sigma_{2}$ a cover of $\Sigma_{1}$ if and only if $\Sigma_{1}^{*}=\Sigma_{2}^{*}$. Covers are just different representations of the same semantics.

It is important to highlight the significance of the implication problem. Suppose the elements of $\Sigma \cup\{\varphi\}$ are considered to be data dependencies that capture important semantic properties. If $\Sigma \models \varphi$ holds, then it suffices to check that any given relation satisfies $\Sigma$ - as this implies that the relation also satisfies $\varphi$. Hence, the validation whether the relation satisfies $\varphi$ is redundant, and a database management system can save considerable resources. Otherwise, if $\Sigma \not \forall \varphi$, then the database management system must validate whether a given relation satisfies $\varphi$ - since this does not follow from the fact that the relation satisfies $\Sigma$.

Note that we have restricted our attention to finite relations only. Therefore, we also study the finite implication problem only. However, for the classes of data dependencies considered in this thesis, the finite implication problem coincides with the unrestricted implication problem, where 
one also permits infinite relations. In general, the finite and unrestricted implication problems are different [1].

\section{Syntactic Inference}

One can attempt to decide the implication problem for a given class $\mathcal{C}$ of data dependencies by a syntactic approach, e.g. by applying inference rules. Popular inference rules have the form

$$
\frac{\text { premise }}{\text { conclusion }} \text { condition, }
$$

and inference rules without any premise are called axioms.

An inference rule is called sound for the implication of data dependencies, if the set of elements of the premise implies the conclusion, given that the condition of the rule is satisfied. For a finite set $\Sigma \cup\{\varphi\}$ of data dependencies and a set $\mathfrak{R}$ of inference rules let $\Sigma \vdash_{\mathfrak{R}} \varphi$ denote the inference of $\varphi$ from $\Sigma$ by $\mathfrak{R}$. That is, there is some sequence $\gamma=\left[\sigma_{1}, \ldots, \sigma_{n}\right]$ of data dependencies such that $\sigma_{n}=\varphi$ and every $\sigma_{i}$ is an element of $\Sigma$ or results from an application of an inference rule in $\mathfrak{R}$ to some elements in $\left\{\sigma_{1}, \ldots, \sigma_{i-1}\right\}$.

For a finite set $\Sigma$ of data dependencies, let $\Sigma_{\mathfrak{R}}^{+}=\left\{\varphi \mid \Sigma \vdash_{\mathfrak{R}} \varphi\right\}$ denote its syntactic closure under inferences by $\mathfrak{R}$. A set $\mathfrak{R}$ of inference rules is said to be sound (complete) for the implication of data dependencies in $\mathcal{C}$ if for every relation schema $R$ and for every set $\Sigma$ of data dependencies in $\mathcal{C}$ over $R$ we have $\Sigma_{\mathfrak{R}}^{+} \subseteq \Sigma^{*}\left(\Sigma^{*} \subseteq \Sigma_{\mathfrak{R}}^{+}\right)$. The (finite) set $\Re$ is said to be a (finite) axiomatization for the implication of data dependencies if $\mathfrak{R}$ is both sound and complete.

In what follows we will focus on two popular classes of data dependencies over relations. That is, we will focus on the class of keys and the class of functional dependencies. In the literature on the relational model only, already approximately 100 different classes of data dependencies have been proposed and investigated. An excellent survey and classification of these classes is the topic of the book [122]. 


\subsubsection{The Class of Keys}

Keys are arguably the most important class of data dependencies [20, 28, 120, 124]. Formally, a key over a relation schema $R$ is an expression key $(K)$ where $K \subseteq R$. A relation $r$ over $R$ is said to satisfy the key key $(K)$ over $R$ if and only if for all tuples $t, t^{\prime} \in r$ the following is true: if $t \neq t^{\prime}$, then there is some $A \in K$ such that $t(A) \neq t^{\prime}(A)$. In other words, a key key $(K)$ is violated by $r$ if there are two different tuples $t, t^{\prime}$ such that $t(K)=t^{\prime}(K)$.

\section{Axiomatization}

The implication problem of keys can be characterized by the set $\mathfrak{K}$ of inference rules in Theorem 2.1][122]. In agreement with the database literature we often write $X Y$ to denote the set union $X \cup Y$.

Theorem 2.1. The following set $\mathfrak{K}$ of inference rules forms a finite axiomatization for the class of keys over relations.

$$
\begin{array}{cc}
\overline{k e y(R)} & \frac{k e y(K)}{k e y\left(K K^{\prime}\right)} \\
\text { (relation axiom) } & \text { (superkey) }
\end{array}
$$

Note that the relation axiom is sound as a relation is defined as a set of tuples, i.e., there cannot be two different tuples that have the same values on all the attributes of the relation schema. In other words, the relation axiom says that no duplicate tuples are permitted in relations. The superkey rule says that every superset of a key is also a key.

As an example consider the set $\Sigma$ over WORK that consists of the key $k e y(E m p)$. The superkey rule allows us to infer the following keys from $\Sigma$ : key (Emp, Dept), key (Emp, Mgr), and key(Emp, Dept, Mgr).

\section{Algorithmic Solution}

The axiomatization provides important hints on how to solve the implication problem of keys algorithmically. That is, to decide whether for a 
given relation schema $R$ and a given set $\Sigma \cup\{k e y(K)\}$ of keys over $R$ it is true that $\Sigma \models k e y(K)$ holds, it is sufficient to check whether $K=R$ or there is some $k e y\left(K^{\prime}\right) \in \Sigma$ such that $K^{\prime} \subseteq K$. If $|X|$ denotes the cardinality of a set $X$, and $\| \Sigma||$ denotes the total number of attributes that occur in $\Sigma$, then the worst-case time-complexity to decide the implication problem of keys over relations is $\mathcal{O}(\max \{|R|,|| \Sigma||\})$.

As an example consider the set $\Sigma=\{k e y(E m p$, Dept $), k e y(E m p, M g r)\}$ of keys over WORK. Consider further the key key(Emp), denoted by $\varphi$. The following relation shows that $\Sigma$ does not imply $\varphi$.

\begin{tabular}{ccc}
\hline Emp & Dept & $M g r$ \\
\hline Dilbert & Information Systems & Gates \\
Dilbert & E-commerce & Jobs \\
\hline
\end{tabular}

Indeed, the singleton Emp does not equal WORK, and is not a superset of any of the keys in $\Sigma$.

The axiomatization $\mathfrak{K}$ shows that one only needs to specify so-called minimal keys. For a set $\Sigma \cup\{k e y(K)\}$ of keys over a relation schema $R$, the key $k e y(K)$ is said to be minimal if and only if $\Sigma \models k e y(K)$ and there is no $k e y\left(K^{\prime}\right) \in \Sigma^{*}$ such that $K^{\prime} \subset K$. Here, $K^{\prime} \subset K$ means that $K^{\prime} \subseteq K$ and $K-K^{\prime} \neq \emptyset$. Intuitively, minimal keys represent minimal numbers of attributes required to distinguish between any two tuples of the database. This is the reason why minimal keys are very important in database practice. Indeed, keys form one of the exclusive classes of data dependencies that are automatically supported by database management systems [33]. The maximum number of minimal keys is exponential in the number of attributes from the underlying relation schema [37].

\subsubsection{The Class of Functional Dependencies}

Keys cannot express many desirable semantic properties that data engineers have in mind. Therefore, more expressive notions of data depen- 
dencies are required in database practice. One of the most popular classes is that of functional dependencies $[5,10,12,28,66,76,86,119,124,129,133]$.

A functional dependency (FD) over a relation schema $R$ is an expression $X \rightarrow Y$ where $X, Y \subseteq R$. A relation $r$ over $R$ satisfies the functional dependency $X \rightarrow Y$ over $R$ if and only if for all $t, t^{\prime} \in r$ the following condition is satisfied: if $t(X)=t^{\prime}(X)$, then $t(Y)=t^{\prime}(Y)$. In other words, the values on $X$ determine the values on $Y$ as a function.

As an example consider the relation schema WORK. The FD Emp $\rightarrow$ Dept expresses the fact that every employee can work for at most one department. The FD Dept $\rightarrow$ Mgr expresses the fact that every department has at most one manager. The relation $r_{\text {total }}$ of Table 2.1 satisfies both of these FDs. However, the relation $r_{\text {total }}$ violates the FD Dept, Mgr $\rightarrow$ Emp: indeed, there are two different employees that work in the same department with the same manager.

Over relations, the class of functional dependencies subsumes the class of keys. That is, for every relation schema $R$, for every attribute set $X \subseteq R$, and for every relation $r$ over $R$ it is true that $r$ satisfies the key key $(X)$ if and only if $r$ satisfies the FD $X \rightarrow R$.

As an example, the relation $r_{\text {total }}$ of Table 2.1] satisfies the key key (Emp) and the FD Emp $\rightarrow$ Dept, Mgr.

\section{Axiomatization}

Armstrong [7] proposed the axiomatization $\mathfrak{F}$ in Theorem 2.2 for the implication of functional dependencies over relations.

Theorem 2.2 (Armstrong, 1974). The following set $\mathfrak{F}$ forms a finite axiomatization for the implication of functional dependencies over relations.

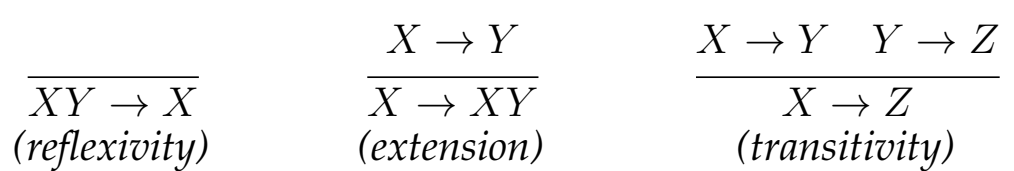


Remark 2.1. The system presented in Theorem 2.2 uses the extension rule instead of the augmentation rule

$$
\frac{X \rightarrow Y}{X Z \rightarrow Y Z}
$$

which was originally proposed by Armstrong [7]. The augmentation rule can be derived from the reflexivity axiom, extension rule, and transitivity rule as follows:

$$
\frac{\frac{\overline{X Z \rightarrow X} \quad X \rightarrow Y}{X Z \rightarrow Y}}{\frac{X Z \rightarrow X Z Y}{X Z Y \rightarrow Y Z}}
$$

As the extension rule is the special case of the augmentation rule where $Z=X$, the soundness and completeness of the reflexivity axiom, augmentation rule, and transitivity rule imply the soundness and completeness of the reflexivity axiom, extension rule, and transitivity rule.

As an example consider the relation schema WORK, and the set $\Sigma$ that consists of the FDs Emp $\rightarrow$ Dept and Dept $\rightarrow$ Mgr. We can infer the FD $E m p$,Dept $\rightarrow$ Dept from the reflexivity axiom. We can infer the FD Emp $\rightarrow$ $M g r$ by applying the transitivity rule to the FDs in $\Sigma$. Moreover, we can infer the FD Emp $\rightarrow E m p, M g r$ by applying the extension rule to the FD Emp $\rightarrow$ Mgr.

\section{Algorithmic Solution}

An important notion is that of a closure $X_{\Sigma}^{*}$ for an attribute set $X \subseteq R$ with respect to a set $\Sigma$ of FDs over $R$. Indeed,

$$
X_{\Sigma}^{*}=\{A \in R \mid \Sigma \models X \rightarrow A\}
$$

consists of all those attributes from $R$ which are functionally determined by $X$ given $\Sigma$. The importance of this notion stems from the fact that for all relation schemata $R$, and all sets $\Sigma \cup\{X \rightarrow Y\}$ of FDs over $R$ it is 
true that $\Sigma \models X \rightarrow Y$ if and only if $Y \subseteq X_{\Sigma}^{*}$ [7, 10]. Hence, to decide the implication problem for the class of FDs it is sufficient and necessary to compute the attribute set closure. A simple, yet efficient algorithm to compute the attribute set closure is Algorithm 2.1] (in [8], but originally presented in [12]) .

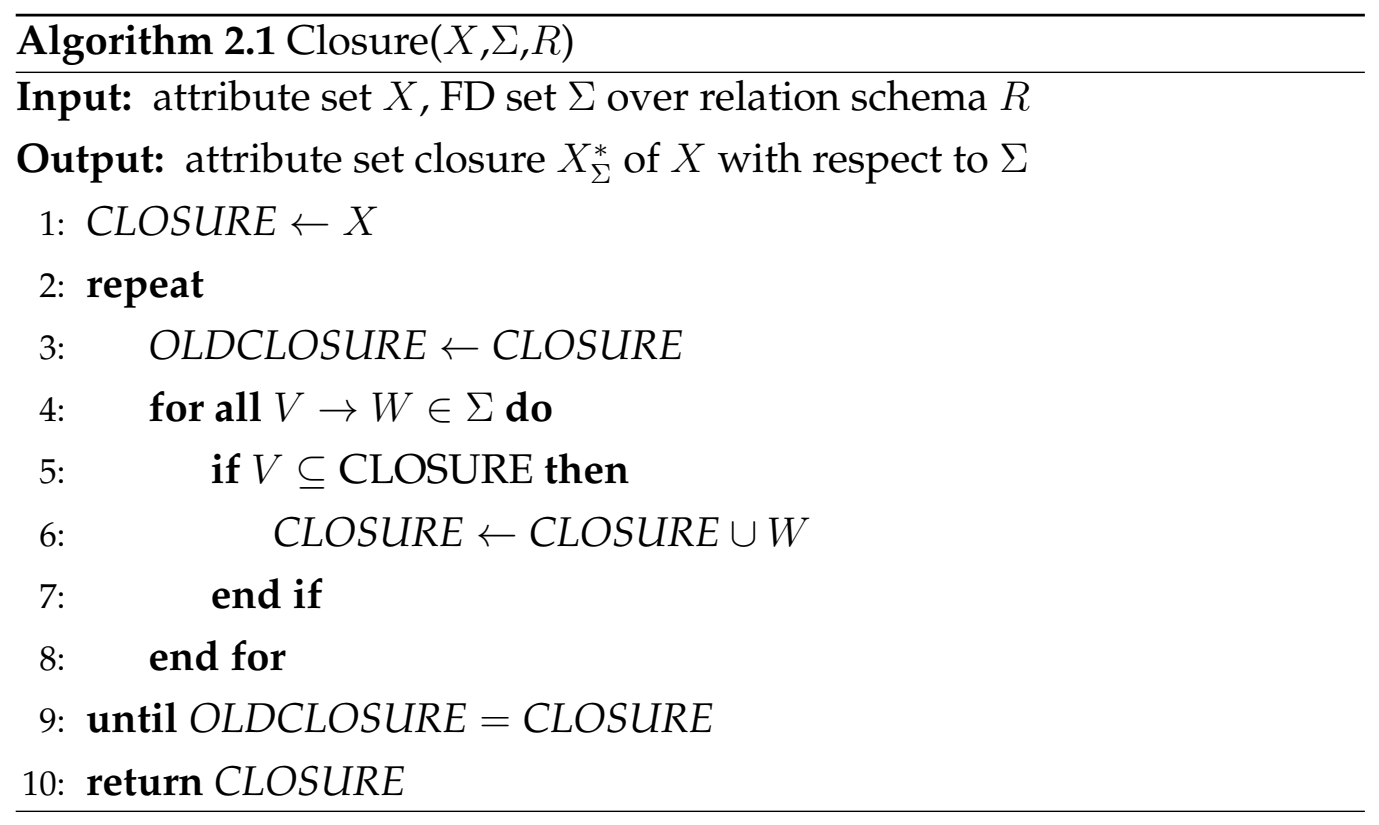

If $\|\Sigma\|$ denotes the total number of attributes that occur in $\Sigma$, the algorithm can be implemented such that the implication problem with input $\Sigma \cup\{X \rightarrow Y\}$ can be decided in time $\mathcal{O}(\|\Sigma \cup\{X \rightarrow Y\}\|)$ [10,40].

As an example, consider the relation schema WORK, the FD set $\Sigma$ that consists of Emp $\rightarrow$ Dept and Dept $\rightarrow M g r$. Let $\varphi$ denote the FD Emp $\rightarrow M g r$. Suppose we would like to decide whether $\Sigma \models \varphi$. Then we declare $X:=$ $\{E m p\}$ and we use Algorithm 2.1 to compute $X_{\Sigma}^{*}=\{$ Emp, Dept, Mgr $\}$. As $\{M g r\} \subseteq X_{\Sigma}^{*}$ it is indeed true that $\Sigma$ implies $\varphi$.

\section{Logical Characterization}

Fagin established an equivalence between the implication problem for the class of functional dependencies over relations and the implication prob- 
lem for the class of Horn clauses in propositional logic [45], that we briefly review here.

For a finite set $L$ of propositional variables, let $L^{*}$ denote the propositional language over $L$, generated from the unary connective $\neg$ (negation), and the binary connective $\vee$ (disjunction). $L^{*}$ is the smallest set that satisfies:

1. $L \subseteq L^{*}$,

2. if $\varphi^{\prime} \in L^{*}$, then $\left(\neg \varphi^{\prime}\right) \in L^{*}$, and

3. if $\varphi_{1}^{\prime}, \varphi_{2}^{\prime} \in L^{*}$, then $\left(\varphi_{1}^{\prime} \vee \varphi_{2}^{\prime}\right) \in L^{*}$.

We assume that negation binds stronger than disjunction, and omit parentheses if it does not cause ambiguity. A literal is a propositional variable or the negation of a propositional variable. A clause over $L$ is an element of $L^{*}$ that is the disjunction of literals. The occurrence of a variable in a clause is negative, if it carries a negation in front of it. Otherwise, the occurrence is said to be positive. A clause over $L$ is a Horn clause if there is at most one positive occurrence of some variable.

An interpretation of $L$ is a total function $\omega: L \rightarrow\{\mathbb{F}, \mathbb{T}\}$ that maps every variable $A^{\prime} \in L$ to its truth value $\omega\left(A^{\prime}\right)$. An interpretation $\omega$ of $L$ can be lifted to a total function $\Omega: \mathcal{L}^{*} \rightarrow\{\mathbb{F}, \mathbb{T}\}$ by means of simple rules:

1. $\Omega\left(A^{\prime}\right):=\omega\left(A^{\prime}\right)$ for all $A^{\prime} \in \mathcal{L}$,

2. $\Omega\left(\neg \varphi^{\prime}\right):=\neg \Omega\left(\varphi^{\prime}\right)$, and

3. $\Omega\left(\varphi^{\prime} \vee \psi^{\prime}\right):=\Omega\left(\varphi^{\prime}\right) \vee \Omega\left(\psi^{\prime}\right)$.

An interpretation $\omega$ is a model of a set $\Sigma^{\prime}$ of formulae in $L^{*}$ if and only if $\Omega\left(\sigma^{\prime}\right)=\mathbb{T}$ holds for every $\sigma^{\prime} \in \Sigma^{\prime}$. We say that $\Sigma^{\prime}$ logically implies an $\mathcal{L}$-formula $\varphi^{\prime}$, denoted by $\Sigma^{\prime} \models_{L} \varphi^{\prime}$, if and only if every interpretation that is a model of $\Sigma^{\prime}$ is also a model of $\varphi^{\prime}$.

For example, for $L=\left\{E m p^{\prime}, D e p t^{\prime}, M g r^{\prime}\right\}$ the set $\Sigma^{\prime}$, consisting of the Horn clauses $\neg E m p^{\prime} \vee D e p t^{\prime}$ and $\neg D e p t^{\prime} \vee M g r^{\prime}$, does not logically imply 
the Horn clause $\varphi^{\prime}=\neg D e p t^{\prime} \vee \neg M g r^{\prime} \vee E m p^{\prime}$. In fact, the interpretation $\omega$ that assigns $\mathbb{F}$ to $E m p^{\prime}$, and $\mathbb{T}$ to both $D e p t^{\prime}$ and $M g r^{\prime}$, is a model of $\Sigma^{\prime}$ but $\operatorname{not} \varphi^{\prime}$.

We assume, without loss of generality, that every FD over $R$ is of the form $X \rightarrow A$ where $A \in R$ (by replacing $X \rightarrow Y$ with the FDs $X \rightarrow A$ for all $A \in Y$ ). Let $\phi: R \rightarrow L$ denote a bijection between the attributes of a relation schema $R$ and the set $L$ of propositional variables. We extend this mapping $\phi$ to a mapping $\Phi$ from functional dependencies over $R$ to Horn clauses over $L$. In fact, for a functional dependency $X \rightarrow A$ over $R$ let $\Phi(X \rightarrow A)=\left(\bigvee_{B \in X} \neg \phi(B)\right) \vee \phi(A)$. In what follows, for a functional dependency $\sigma$ we write $\sigma^{\prime}$ instead of $\Phi(\sigma)$, and for a finite set $\Sigma$ of FDs we write $\Sigma^{\prime}$ instead of $\left\{\sigma^{\prime} \mid \sigma \in \Sigma\right\}$.

Theorem 2.3. Let $R$ be a relation schema, and let $\Sigma \cup\{\varphi\}$ denote a set of FDs over $R$. Then $\Sigma$ implies $\varphi$ if and only if $\Sigma^{\prime}$ logically implies $\varphi^{\prime}$.

For example, the set $\Sigma$, consisting of the FDs Emp $\rightarrow$ Dept and Dept $\rightarrow$ $M g r$, does not imply the FD $\varphi=\{$ Dept, Mgr $\} \rightarrow E m p$, illustrated by the two-tuple relation $r$ :

\begin{tabular}{ccc}
\hline Emp & Dept & $M g r$ \\
\hline Dilbert & Information Systems & Gates \\
Alice & Information Systems & Gates \\
\hline
\end{tabular}

Indeed, we have already seen above that the associated set $\Sigma^{\prime}$ of Horn clauses does not logically imply the associated Horn clause $\varphi^{\prime}$.

The example also illustrates how counterexample relations to the implication of FDs are in one-to-one correspondence to counterexample truth assignments to the logical implication of the associated Horn clauses. Indeed, the special truth assignment $\omega_{r}$ assigns true to the variable $A^{\prime}$ when the two tuples in $r$ have matching values on the attribute $A$. Theorem 2.3 follows now easily from the fact that for every $\operatorname{FD} \varphi$ that is not implied by an FD set $\Sigma$, there is a two-tuple relation that satisfies all FDs in $\Sigma$ and violates $\varphi$ [45]. 


\section{Fundamental Assumption}

Functional dependencies play a central role for the design [24, 54, 105, 107 , 123, 124] and normalization [90, 106] of databases, and have found important applications in a diverse number of areas including query optimization [27, 39, 104], database maintenance [53, 74, 118] and security [14, 132], and data cleaning [51, 52], entry [105], exchange [98] and integration [23, 101, 131]. Fundamental to all these applications is the assumption that the set of functional dependencies that are semantically meaningful for a given relation schema $R$ has been correctly identified.

\subsection{Armstrong Relations}

It is an important question to ask how database designers and data engineers can learn the set of functional dependencies that are semantically meaningful for a given relation schema $R$. Intuitively, humans learn a lot from good examples. It is therefore reasonable to assume that designers and engineers can learn meaningful functional dependencies from good sample data. The question arises what sample data qualifies as good. For a set $\Sigma$ of functional dependencies, a good relation should at least satisfy $\Sigma$. As a consequence, every good relation will also satisfy every functional dependency implied by $\Sigma$, i.e., $\Sigma^{*}$. A perfect relation violates every other functional dependency. That is, a perfect relation for $\Sigma$ satisfies $\Sigma$ and violates every functional dependency not implied by $\Sigma$.

It is a consequence of a result by Armstrong [7] that for all relation schemata $R$ and for all sets $\Sigma$ of FDs over $R$ there is a perfect sample relation for $\Sigma$. Following common terminology we call such a relation an Armstrong relation for $\Sigma$ [48]. The following example illustrates the potential benefits of utilizing Armstrong relations for the discovery of semantically meaningful FDs.

Let us assume we develop an information system for some human re- 
source application. So far, we have defined the relation schema WORK. Suppose the FDs Emp $\rightarrow$ Dept and Dept $\rightarrow$ Mgr have been identified as semantically meaningful for the application. They form the FD set $\Sigma$. However, it is unclear whether there are any other semantically meaningful FDs that have not been identified yet. Therefore, we decide to inspect a relation that faithfully represents our initial design draft. The relation we decide to examine is the one in Table 2.2. This relation is Armstrong for the FD set $\Sigma$.

\begin{tabular}{ccc}
\hline Emp & Dept & $M g r$ \\
\hline Dilbert & Information Systems & Gates \\
Alice & Information Systems & Gates \\
Wally & E-Commerce & Gates \\
Dogbert & Library studies & Jobs \\
\hline
\end{tabular}

Table 2.2: The Armstrong relation $r_{\text {Arm }}$ for $\Sigma$

By inspecting the Armstrong relation the designers simply notice that Gates manages two different departments, i.e. Information Systems and ECommerce. After consultation with the end users of the human resource application, we decide to specify the FD Mgr $\rightarrow$ Dept. That is, in the application domain every manager can manage at most one department.

This simple example illustrates the potential benefit of using Armstrong relations in the process of discovering the complete set of FDs that are semantically meaningful for the underlying application domain. Failure to identify such a complete set means that the output of the requirements analysis is afflicted with errors.

Empirical studies show that more than half the errors which occur during systems development are requirements errors [42, 78, 99]. Requirements errors are also the most common cause of failure in systems development projects [42, 114, 115]. The cost of errors increases exponentially over the development life cycle: it is more than 100 times more costly to 
correct a defect post-implementation than it is to correct it during requirements analysis [16]. This suggests that it would be more effective to concentrate quality assurance efforts in the requirements analysis stage, in order to catch requirements errors as soon as they occur, or to prevent them from occurring altogether [136]. Hence, Armstrong relations appear to be an invaluable tool for the requirements analysis of the target database.

Indeed, Armstrong relations are widely regarded as good test data and a helpful tool for data engineers to judge, justify, convey, or test their understanding of the relation schema [3, 11, 13, 35, 36, 96]. In fact, an FD is implicit in the explicit specification of an FD set $\Sigma$ if and only if it is satisfied by an Armstrong relation for $\Sigma$. For this reason, Armstrong relations represent one of the few instances where example-based reasoning is effective. That is, if $\Sigma$ constitutes the design choice of the set of FDs currently perceived as meaningful to the underlying application domain, then an Armstrong relation $r_{\Sigma}$ for $\Sigma$ constitutes an example on which data engineers can test the meaningfulness of an arbitrary FD $\varphi$. Namely, $\varphi$ is meaningful under the current design choice if and only if the example relation $r_{\Sigma}$ satisfies $\varphi$. Consequently, this approach to design is called designby-example. Industry-leading data modeling tools, such as the ERwin data modeler, emphasize the need for good test data to validate the semantics of the models they produce [22].

\subsubsection{General Definition of an Armstrong Relation}

For future reference we repeat the formal definition of an Armstrong relation here [47, 48].

Let $\mathcal{C}$ denote a class of data dependencies, let $R$ denote a relation schema, and $\Sigma$ a set of data dependencies in $\mathcal{C}$ over $R$. We say that a relation $r$ over $R$ is a $\mathcal{C}$-Armstrong relation for $\Sigma$ if and only if $r$ satisfies $\Sigma$ and violates every data dependency in $\mathcal{C}$ that is not implied by $\Sigma$. We say that $\mathcal{C}$ enjoys Armstrong relations if and only if for every relation schema $R$, and every 
set $\Sigma$ of data dependencies in $\mathcal{C}$ over $R$ there is a relation over $R$ that is Armstrong for $\Sigma$.

\subsubsection{Armstrong Relations for Functional Dependencies}

We now review the existing theory of Armstrong relations for the class of functional dependencies [11, 96].

\section{Structural Properties}

Armstrong showed that the class of functional dependencies over relations does enjoy Armstrong relations [7]. Characteristics of these Armstrong relations involve three different notions. The first notion is that of a closed attribute set [11]. Let $R$ be a relation schema, and $\Sigma$ a set of FDs over $R$. We say that an attribute set $X \subseteq R$ is closed with respect to $\Sigma$ if and only if $X_{\Sigma}^{*}=X$. Furthermore, let $c l_{\Sigma}(R)$ denote the set of all attribute subsets of $R$ that are closed with respect to $\Sigma$. The function $(\cdot)_{\Sigma}^{*}: \mathcal{P}(R) \rightarrow \mathcal{P}(R)$ defined by taking any attribute set $X \subseteq R$ to its attribute set closure $X_{\Sigma}^{*}$ with respect to $\Sigma$ indeed defines a closure operation. That is, it is extensive $\left(X \subseteq X_{\Sigma}^{*}\right.$ ), increasing (for $X \subseteq Y$ we have $X_{\Sigma}^{*} \subseteq Y_{\Sigma}^{*}$ ) and idempotent $\left(\left(X_{\Sigma}^{*}\right)_{\Sigma}^{*}=X_{\Sigma}^{*}\right)$.

As an example consider Table 2.3 which shows a closure operation $(\cdot)_{\Sigma}^{*}$ for $\Sigma=\{E m p \rightarrow$ Dept,Dept $\rightarrow M g r\}$. The closed attribute sets of WORK are therefore $\emptyset,\{M g r\},\{$ Dept, Mgr $\}$ and $\{$ Emp, Dept, Mgr $\}$.

The second notion is that of a maximal set [96]. A set $X \subseteq R$ is said to be maximal for an attribute $A \in R$ with respect to a set $\Sigma$ of FDs over $R$ if and only if $\Sigma \not \forall X \rightarrow A$ and for every attribute $B \in R-X$ it is true that $\Sigma \models X B \rightarrow A$. Hence, $X$ is maximal among all those attribute sets that do not functionally determine $A$, given $\Sigma$. Let $\max _{\Sigma}(A)$ denote the set of all attribute sets that are maximal for $A$ with respect to $\Sigma$. Further, let $\max _{\Sigma}(R)=\bigcup_{A \in R} \max _{\Sigma}(A)$ denote the maximal sets of $R$.

The third notion is that of an agree set. Given two tuples $t$ and $t^{\prime}$ over 


\begin{tabular}{|c|c|}
\hline \multicolumn{2}{|c|}{$(\cdot)_{\Sigma}^{*}$} \\
\hline$X$ & $X_{\Sigma}^{*}$ \\
\hline$\emptyset$ & $\emptyset$ \\
Eept & Emp, Dept, Mgr \\
$\mathbf{M g r}$ & Dept, Mgr \\
Emp, Dept & Emp , Dept, Mgr \\
Emp, Mgr & Emp, Dept, Mgr \\
Dept, Mgr & Dept, Mgr \\
Emp, Dept, Mgr & Emp, Dept, Mgr \\
\hline
\end{tabular}

Table 2.3: Closure operation $(\cdot)_{\Sigma}^{*}$ for $\Sigma=\{E m p \rightarrow$ Dept; Dept $\rightarrow$ Mgr $\}$. The closed header sets of WORK are printed in bold

\begin{tabular}{|c|c|}
\hline \multicolumn{2}{|c|}{$\max _{\Sigma}($ WORK $)$} \\
\hline$A \in R$ & $\max _{\Sigma}(A)$ \\
\hline Emp & $\{$ Dept,$M g r\}$ \\
Dept & $\{M g r\}$ \\
$M g r$ & $\emptyset$ \\
\hline
\end{tabular}

Table 2.4: Families of maximal sets of WORK for $\Sigma=\{E m p \rightarrow$ Dept; Dept $\rightarrow$ Mgr 
$R$, the agree set $\operatorname{ag}\left(t, t^{\prime}\right)=\left\{A \in R \mid t(A)=t^{\prime}(A)\right\}$ consists of all those attributes of $R$ on which $t$ and $t^{\prime}$ agree, i.e., have the same value [11.96]. Now, the agree set $a g(r)$ of a relation $r$ is the set of the agree sets of all pairs of distinct tuples in $r$. That is, $a g(r)=\left\{a g\left(t, t^{\prime}\right) \mid t, t^{\prime} \in r\right.$ and $\left.t \neq t^{\prime}\right\}$.

As an example consider the Armstrong relation $r_{\text {Arm }}$ for

$$
\Sigma=\{\text { Emp } \rightarrow \text { Dept,Dept } \rightarrow M g r\}
$$

from Table 2.2. The agree sets of this relation are $\{$ Dept, $M g r\},\{M g r\}, \emptyset$.

We can now state the anticipated structural characterization of Armstrong relations [11,96].

Theorem 2.4. Let $R$ be a relation schema, $\Sigma$ a set of FDs and $r$ a relation over $r$. Then $r$ is an Armstrong relation for $\Sigma$ if and only if the following condition is satisfied:

$$
\max _{\Sigma}(R) \subseteq \operatorname{ag}(r) \subseteq \mathrm{cl}_{\Sigma}(R) .
$$

The condition $\max _{\Sigma}(R) \subseteq \operatorname{ag}(r)$ means that every maximal set is an agree set of the relation. This ensures that the relation violates all FDs not implied by $\Sigma$. The condition $a g(r) \subseteq c l_{\Sigma}(R)$ means that every agree set is closed. This ensures that $r$ satisfies $\Sigma$.

As an example, consider again the relation schema WORK with FD set $\Sigma=\{E m p \rightarrow$ Dept, Dept $\rightarrow M g r\}$. As a summary of the previous examples we have

\begin{tabular}{|c|c|}
\hline $\max _{\Sigma}($ WORK $)$ & $\{$ Dept,$M g r\},\{M g r\}, \emptyset$ \\
\hline$a g\left(r_{\text {Arm }}\right)$ & $\{$ Dept,$M g r\},\{M g r\}, \emptyset$ \\
\hline$c l_{\Sigma}($ WORK $)$ & $\{$ Dept,$M g r\},\{M g r\}, \emptyset,\{$ Emp, Dept,$M g r\}$ \\
\hline
\end{tabular}

We conclude that $r_{A r m}$ is indeed an Armstrong relation for $\Sigma$.

\section{Computational Properties}

Theorem 2.4 provides a strategy for computing an Armstrong relation for a given set $\Sigma$ of FDs over a given relation schema $R$ : Compute the set of 
maximal sets with respect to $\Sigma$, and generate pairs of tuples whose agree sets realize these maximal sets. As every maximal set is closed, this strategy produces an Armstrong relation. The remainder of this section is used to develop this strategy in detail.

Let $R$ be a relation schema, and $\Sigma=\Sigma^{\prime} \cup\{X \rightarrow A\}$ be a set of FDs over $R$. For $W C \subseteq R$, it takes $\mathcal{O}(|R| \times|| \Sigma||)$ time to test whether $W \in \max _{\Sigma}(C)$. We use $\operatorname{mtest}(W, C, R, \Sigma)$ to denote the test if $W \in \max _{\Sigma}(C)$. The maximal sets for $R$ with respect to $\Sigma$ can be computed by testing all subsets of $R$. This, however, will hardly be efficient. The following result establishes an iterative approach for computing the maximal sets for $R$ with respect to $\Sigma$.

Let $R$ be a relation schema, and $\Sigma=\Sigma^{\prime} \cup\{X \rightarrow A\}$ a set of FDs over $R$. For $C \in R$ let $V \in \max _{\Sigma}(C)$. Then either $V \in \max _{\Sigma^{\prime}}(C)$ or for some $B \in X$, $Z \in \max _{\Sigma^{\prime}}(B)$ and $W \in \max _{\Sigma^{\prime}}(C)$ we have $V=W \cap Z$ [96].

As an example, consider the relation schema WORK with FD set $\Sigma=$ $\{E m p \rightarrow$ Dept,Dept $\rightarrow M g r\}$. The following table shows the maximal set families for all attributes of WORK with respect to increasing sets of functional dependencies.

\begin{tabular}{|c|c|c|c|}
\hline$A \in R$ & $W \in \max _{\emptyset}(A)$ & $W \in \max _{\{\text {Emp } \rightarrow \text { Dept }\}}(A)$ & $W \in \max _{\Sigma}(A)$ \\
\hline Emp & $\{$ Dept, Mgr $\}$ & $\{$ Dept, Mgr $\}$ & $\{$ Dept, Mgr $\}$ \\
Dept & $\{$ Emp, Mgr $\}$ & $\{M g r\}$ & $\{M g r\}$ \\
$M g r$ & $\{$ Emp, Dept $\}$ & $\{$ Emp, Dept $\}$ & $\emptyset$ \\
\hline
\end{tabular}

Note, in particular, that $\emptyset \in \max _{\Sigma}(M g r)$ satisfies $\emptyset=V=W \cap Z$ for $Z=\{M g r\}$ and $W=\{$ Emp, Dept $\}$.

Algorithm 2.2 computes the families of maximal sets [97]. It starts with the maximal sets for $R$ with respect to an empty FD set, and then adds the FDs of $\Sigma$ one by one while monitoring the resulting changes to the family of maximal sets.

Algorithm 2.3 computes an Armstrong relation $r$ for a set $\Sigma$ of FDs [96]. First, it computes the families $\max _{\Sigma}(R)$ of maximal sets using Algorithm 2.2. Subsequently, it produces a relation $r$ such that $\max _{\Sigma}(R) \subseteq a g(r)$ holds. 


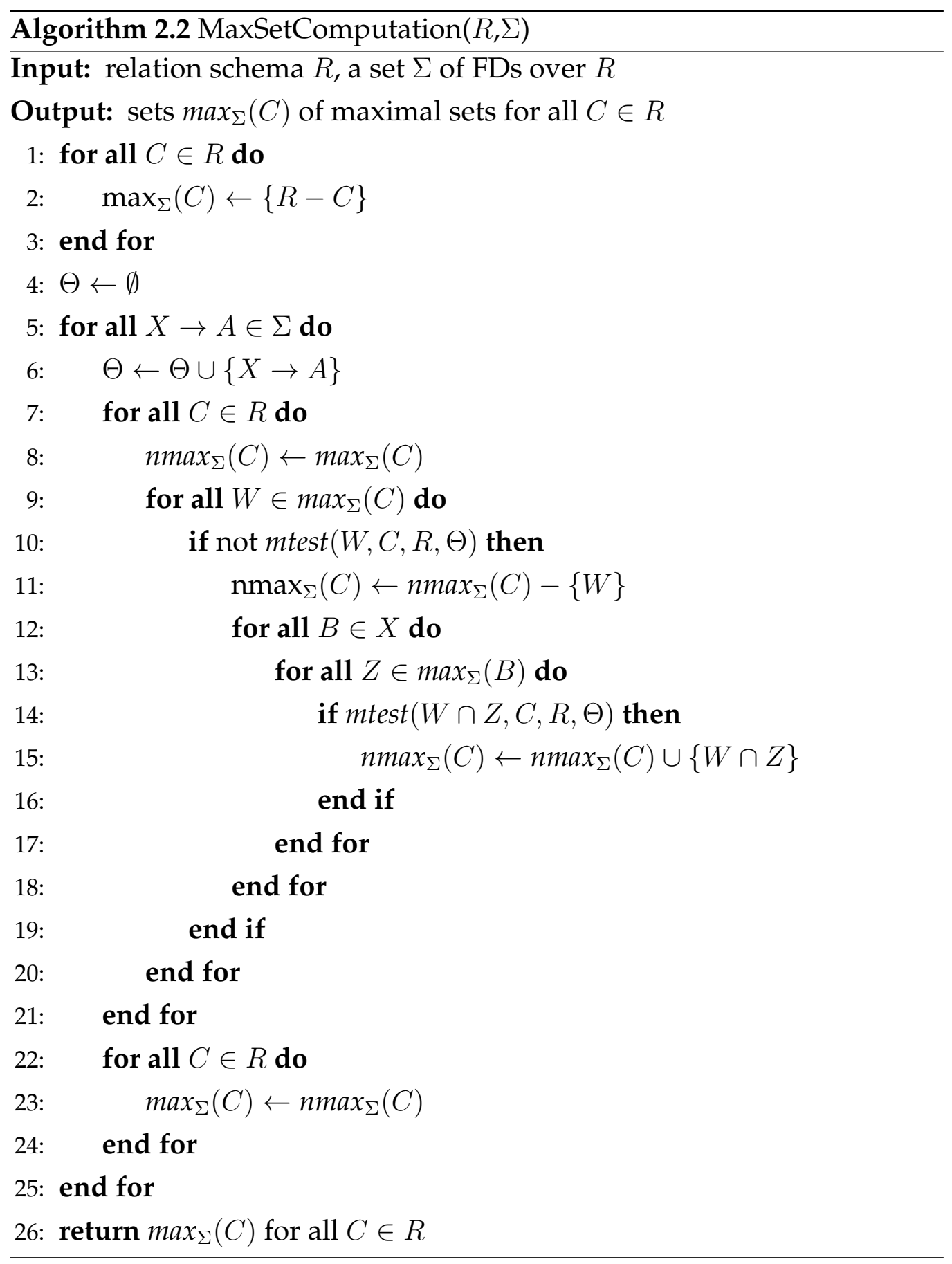




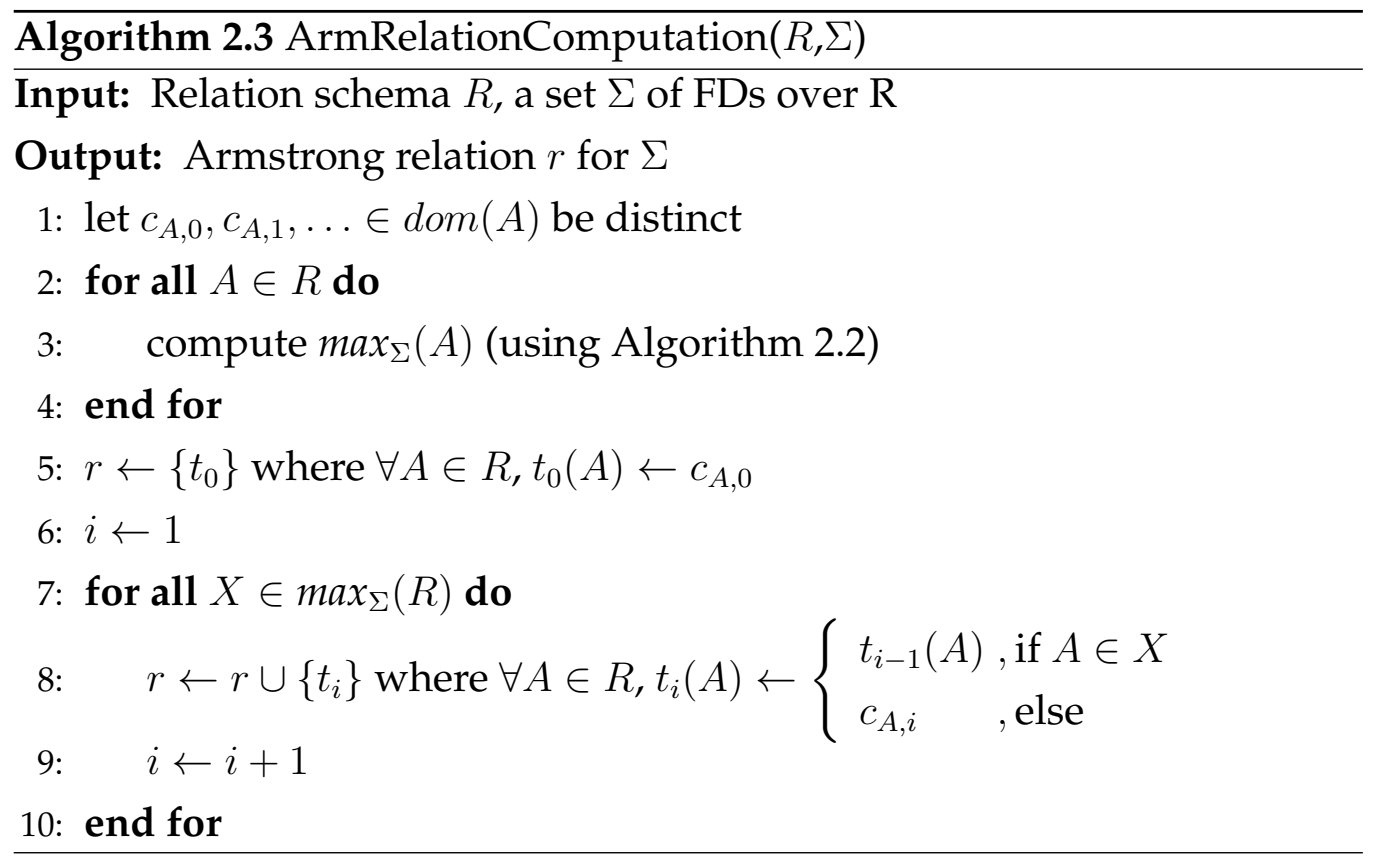

As an example, Algorithm 2.3 is applied to compute an Armstrong relation for $\Sigma=\{E m p \rightarrow$ Dept,Dept $\rightarrow M g r\}$ over the relation schema WORK. Table 2.5 shows this relation. When substituted adequately, this relation yields the one in Table 2.2 .

\begin{tabular}{ccc}
\hline Emp & Dept & $M g r$ \\
\hline$c_{\text {Emp }, 0}$ & $c_{\text {Dept }, 0}$ & $c_{M g r, 0}$ \\
$c_{\text {Emp }, 1}$ & $c_{\text {Dept }, 0}$ & $c_{M g r, 0}$ \\
$c_{\text {Emp }, 2}$ & $c_{\text {Dept }, 2}$ & $c_{M g r, 0}$ \\
$c_{\text {Emp }, 3}$ & $c_{\text {Dept }, 3}$ & $c_{M g r, 3}$ \\
\hline
\end{tabular}

Table 2.5: An Armstrong relation for WORK computed by Algorithm 2.3

The complexity of finding an Armstrong relation, given a set of functional dependencies over $R$, is precisely exponential in the size $|R|$ of $R$. We recall what we mean by precisely exponential [11]. Firstly, it means that there is an algorithm for computing an Armstrong relation, given a set $\Sigma$ 
of FDs, where the running time of the algorithm is exponential in the number of attributes. Secondly, it means that there is a set $\Sigma$ of FDs in which the number of tuples in each minimum-sized Armstrong relation for $\Sigma$ is exponential - thus, an exponential amount of time is required in this case simply to write down the relation [11, 96].

Despite the general worst-case exponential complexity in the number of attributes, Algorithm 2.3 is a fairly simple algorithm for generating Armstrong relations that is quite conservative in its use of time.

Let the size of an Armstrong relation be defined as the number of tuples that it contains. In practice, the most appealing Armstrong relation for an FD set $\Sigma$ should be of minimum size. The reason is that a small number of tuples is easier to comprehend for humans. Therefore, it is a practical question to ask how many tuples a minimum-sized Armstrong relation requires. An Armstrong relation $r$ for $\Sigma$ is said to be minimumsized if there is no Armstrong relation $r^{\prime}$ for $\Sigma$ such that $\left|r^{\prime}\right|<|r|$. That is, for a minimum-sized Armstrong relation for $\Sigma$ there is no Armstrong relation for $\Sigma$ with a smaller number of tuples [11].

Theorem 2.5. Let $\Sigma$ be a set of FDs over relation schema $R$, and let $r$ be a minimum-sized Armstrong relation for $\Sigma$. Then

$$
\frac{\sqrt{1+8 \cdot\left|\max _{\Sigma}(R)\right|}}{2} \leq|r| \leq\left|\max _{\Sigma}(R)\right|+1 .
$$

We conclude that Algorithm 2.3 always computes an Armstrong relation of reasonably small size. On input $(R, \Sigma)$, Algorithm 2.3 computes an Armstrong relation for $\Sigma$ whose size is at most quadratic in the size of a minimum-sized Armstrong relation for $\Sigma[11,96]$.

\section{Prototypes}

Silva and Melkanoff [112] were the first to recognize the "practical" potential of Armstrong relations for the discovery of semantically meaningful dependencies. They implemented a prototype that presents a design team 
with an Armstrong relation for a given set of functional and multivalued dependencies. Their idea was that with the help of an Armstrong relation the design team did not have to think about whether some dependency is implied by the input, but simply notice whether the dependency is satisfied or violated by the relation. Noticeably, for the class of FDs this capability is also included in the Database Design Expert System DBE [15], and for the class of standard FDs and inclusion dependencies such a functionality is also provided by the DBA companion [35]. Specifically, the algorithms for the computation of Armstrong relations presented in this section have been implemented in the Design-by-example tool available from http://www.cs.uta.fi/ hs/dbe.html.

\subsubsection{Evidence For the Usefulness of Armstrong Relations}

In previous work, Armstrong relations were called "user-friendly representations" of sets of data dependencies [96], and it was stated that they are "useful for database design" [9,96]. However, the phrase "useful for database design" was exclusively justified in terms of the structural and algorithmic properties of Armstrong relations. For instance, this may refer to the fact that FDs enjoy Armstrong relations. Other interpretations of "useful" may refer to either the size of an Armstrong relation for an FD set $\Sigma$, or the existence/efficiency of algorithms to compute such an Armstrong relation. These interpretations of "useful" have received considerable interest from the research community [11, 36, 63, 96], as demonstrated on the previous pages.

Langeveldt and Link [77] conducted a first empirical investigation into the usefulness of Armstrong relations for the discovery of semantically meaningful functional dependencies. Specifically, they introduced the three measures of soundness, completeness, and proximity to study the additional insights that humans can obtain from the inspection of Armstrong relations. Their first main result indicates that Armstrong relations are 
not useful in terms of soundness, i.e., in using Armstrong relations it is not more likely to recognize meaningless FDs which are incorrectly perceived as meaningful. Their second main result indicates that Armstrong relations are indeed useful in terms of completeness, i.e., in using an Armstrong relation it is more likely to recognize meaningful FDs that are incorrectly perceived as meaningless. Their results are intuitive as it seems unlikely to recognize the satisfaction of semantically meaningless FDs, but it seems relatively likely to recognize the violation of semantically meaningful FDs by the given Armstrong relation.

The study by Langeveldt and Link [77] is important for a number of reasons. It is the first research that shows how Armstrong databases are effectively exploited by humans. In fact, the results demonstrate how Armstrong databases can help database designers identify a more complete set of requirements for the target database. The design quality of the target database is greatly affected by the level of completeness. After all, normalization algorithms and de-normalization strategies can only work effectively, when the business rules of the application domain have been successfully acquired. In turn, the design quality of the target database has a large impact on the performance of the business that the database supports. Finally, the work of [77] has provided a first framework for the empirical evaluation of the usefulness of Armstrong databases in terms of different measures. It will be one of the goals in this thesis to extend this framework towards the classes of uniqueness constraints and functional dependencies over partial databases, whose interaction is more involved than that of their counterparts over pure relations.

\subsubsection{Constraint Acquisition by Sample Data and Natural Language Processing}

The article [2] proposes an informal approach to identify semantic constraints by using natural language processing and sample data. Semantic 
constraints considered in this article comprise keys, functional dependencies, cardinality constraints, inclusion, and exclusion dependencies. The authors derive the structural and semantical part of the future database by exploiting targeted dialogues in natural language. The output of this process is evaluated in reasons of ambiguity and fuzzyness of the natural language. The structural part can be validated with the help of a graphical editor, and the derived semantics is discussed with the help of sample relations. In particular, the authors propose useful heuristics that minimize the search space for possible semantic constraints. The approach has also been implemented as part of a larger database design system called Rapid Application and Database Development (RADD). It is further noted that the discovery of semantic constraints from sample databases, the heuristics, and the proposed informal validation methods are also useful in reverse engineering. Indeed, the techniques can be exploited to discover the semantic constraints that are valid in a given database, which will significantly assist the translation of an existing database into other data models.

\subsection{Partial Information in Databases}

One of the most important extensions of Codd's basic relational model [28] is partial information. This is mainly due to the high demand for the correct handling of such information in real-world applications. Approaches to deal with partial information comprise partial relations [30, 72, 87], orrelations [73, 88, 126] or fuzzy relations [113]. Here we focus on partial relations.

In the literature, many interpretations of null markers have been proposed; for example, "missing" or "value unknown at present" [29, 61, 62], "non-existence" [95], "inapplicable" [62], "no information" [134] and "open" [60]. In this research, two popular interpretations of null markers will be considered: no information and value unknown at present. 


\begin{tabular}{ccc}
\hline Emp & Dept & $M g r$ \\
\hline Dilbert & Information Systems & Gates \\
Alice & Information Systems & Gates \\
$\mathrm{ni}$ & E-commerce & Jobs \\
\hline
\end{tabular}

Table 2.6: The partial relation $r_{n i}$ over WORK

\subsubsection{No Information}

SQL allows occurrences of a single null marker [33]. For this reason the null marker should be able to express any kind of partial information about domain values wherever it occurs. That means, a domain value might not exist at all, or a domain value does exist but is currently unknown. In the literature this interpretation of a null marker is known as no information [134]. To encompass this interpretation of a null marker it is assumed that the marker $n i$ is a distinguished element in the domain of each attribute. Furthermore, relations that may feature occurrences of a null marker are also called partial relations. For $X \subseteq R$, a tuple $t$ over relation schema $R$ is said to be $X$-total, if $t(A) \neq \mathrm{ni}$ for all $A \in X$. A partial relation is said to be $X$-total, if every of its tuples is $X$-total.

As an illustration consider Table 2.6 which shows a partial relation $r_{\mathrm{ni}}$ over WORK. The last tuple features the ni marker at the Emp attribute. This means that either the employee for this tuple does not exist, or the employee does exist but is unknown at present.

It is possible for a partial relation to have a tuple whose information content subsumes that of another tuple in the same partial relation. For example, we may extend the partial relation $r_{n i}$ of Table 2.6 by another tuple, say

(Wally, E-commerce, Jobs).

The information content of the new tuple subsumes that of the third tuple ( $\mathrm{ni}, \mathrm{E}$-commerce, Jobs) in $r_{n i}$. One may argue that a partial relation 


\begin{tabular}{ccc}
\hline Emp & Dept & $\mathrm{Mgr}$ \\
\hline Dilbert & Information Systems & Gates \\
Alice & Information Systems & Gates \\
unk & E-commerce & Jobs \\
\hline
\end{tabular}

Table 2.7: The Codd relation $r_{\text {unk }}$ over WORK

should only feature tuples with a maximal information content. On the other hand, the removal of subsumed tuples from a partial relation may be too costly to implement in real database systems [33, 122].

More formally, a tuple $t$ over $R$ subsumes a tuple $t^{\prime}$ over $R$, denoted by $t^{\prime} \sqsubseteq t$, if and only if for all $A \in R$ it is true that $t^{\prime}(A)=n i$ or $t^{\prime}(A)=t(A)$ [89]. A partial relation $r$ is said to be subsumption-free if and only if there are no two tuples $t, t^{\prime} \in r$ such that $t^{\prime} \neq t$ and $t^{\prime} \sqsubseteq t$ hold.

\subsubsection{Value Unknown at Present}

Codd [30] suggested originally to handle incomplete information by adding the single null marker unk to the domains of attributes. The meaning of each occurrence of unk is "value unknown at present". Partial relations which can feature occurrences of unk will be called Codd relations. Partial relations which can feature occurrences of $\mathrm{ni}$ will be called $S Q L$ relations. In the following we use the term partial relations to express that a certain fact holds for Codd and for SQL relations.

The null marker unk is quite different from the null marker ni. As an illustration consider Table 2.7 which shows a Codd relation over WORK. The last tuple features the unk marker at the Emp attribute. This means that the employee for this tuple does exist but is unknown at present.

In the literature, the semantics of Codd relations has been investigated [86, 87]. A popular approach is that of a possible world semantics. Intuitively, a possible world of a Codd relation results from replacing each 


\begin{tabular}{ccc}
\hline Emp & Dept & $M g r$ \\
\hline Dilbert & Information Systems & Gates \\
Alice & Information Systems & Gates \\
Wally & E-commerce & Jobs \\
\hline
\end{tabular}

Table 2.8: A possible world relation for $r_{\text {unk }}$

occurrence of the unk marker by a value from the domain of the attribute where it occurs. More formally, the set of all possible worlds relative to a Codd relation $r$ over $R$, denoted by $\operatorname{Poss}(r)$, is defined by

$$
\begin{aligned}
\operatorname{Poss}(r):= & \left\{r^{\prime} \mid r^{\prime} \text { is a relation over } R \text { and there is a bijection } b: r \rightarrow r^{\prime}\right. \\
& \text { such that } \forall t \in r, t \text { is subsumed by } b(t) \text { and } b(t) \text { is } R \text {-total }\} .
\end{aligned}
$$

This definition of possible worlds embodies the closed world assumption (CWA) [72], since only $R$-total tuples from the relation $r$ can be present in $\operatorname{Poss}(r)$. Table 2.8 shows a relation that is a possible world for the Codd relation $r_{\text {unk }}$ of Table 2.7

\subsection{Data Dependencies over Partial Relations}

This section contains a review of previous work on data dependencies over SQL and Codd relations. We will focus on those findings that are of relevance for this research.

\subsubsection{Functional Dependencies over SQL Relations}

Lien [89] pioneered research on the class of functional dependencies over subsumption-free SQL relations. We summarize his results on the implication problem associated with this class.

A functional dependency with nulls (NFD) over a relation schema $R$ is an expression $X \rightarrow Y$ where $X, Y \subseteq R$. An SQL relation $r$ over $R$ satisfies 


\begin{tabular}{ccc}
\hline Emp & Dept & Mgr \\
\hline Dilbert & $\mathrm{ni}$ & Gates \\
Dilbert & $\mathrm{ni}$ & Jobs \\
Wally & E-commerce & Gates \\
\hline
\end{tabular}

Table 2.9: The SQL relation $r_{S Q L}$ over WORK

the FD $X \rightarrow Y$ if and only if for all tuples $t, t^{\prime} \in r$ the following holds: if $t(X)=t^{\prime}(X)$ and $t, t^{\prime}$ are both $X$-total, then $t(Y)=t^{\prime}(Y)$.

Hence, whenever two tuples agree on a non-null restriction to $X$, then they also agree on the restriction to $Y$, which may be partial. In other words, an NFD $X \rightarrow Y$ can only be violated by an SQL relation $r$ if there are two distinct $X$-total tuples $t, t^{\prime} \in r$ such that $t(X)=t^{\prime}(X)$ and there is some attribute $A \in Y$ such that $t(A) \neq t^{\prime}(A)$ (i.e. either $t(A)$ and $t^{\prime}(A)$ have different non-null values, or one of $t(A)$ and $t^{\prime}(A)$ is null but the other value is non-null).

As examples, the SQL relation $r_{S Q L}$ in Table 2.9 satisfies the NFDs Emp $\rightarrow$ Dept and Dept $\rightarrow$ Mgr, and it violates the NFDs Mgr $\rightarrow$ Dept, Mgr $\rightarrow$ Emp, and $\operatorname{Emp} \rightarrow M g r$.

For total SQL relations the definition of an NFD reduces to that of an FD, and so is a correct generalization of the concept. It is also consistent with the no information interpretation [8]. Firstly, tuples with nulls in attributes in $X$ cannot cause a violation of a functional dependency $X \rightarrow$ $Y$ : the nulls mean that no information is available about those attributes. Secondly, the functional dependence of $Y$ on $X$ forces any two $X$-total tuples $t, t^{\prime}$ where $t(X)=t^{\prime}(X)$ to have the same information on all the attributes in $Y$. That is, for all $A \in Y$ we have either $t(A)=\mathrm{ni}=t^{\prime}(A)$ or $\mathrm{ni} \neq t(A)=t^{\prime}(A) \neq \mathrm{ni}$.

Next Lien's axiomatization and algorithmic solution of the implication problem for the class of NFDs is revisited. 


\section{Axiomatization}

It is noted first that the transitivity rule for FDs from Theorem 2.2 is not sound for the implication of NFDs. A simple example is presented in Table 2.9 . Indeed, the relation $r_{S Q L}$ satisfies the NFDs Emp $\rightarrow$ Dept and Dept $\rightarrow$ $M g r$, but it violates the NFD Emp $\rightarrow$ Mgr. It follows that the implication problem of NFDs is different from that of FDs.

Theorem 2.6 (Lien,1982). The set $\mathfrak{L}$, consisting of reflexivity axiom, augmentation rule, decomposition rule, and union rule below, forms a finite axiomatization for the class of NFDs over SQL relations.

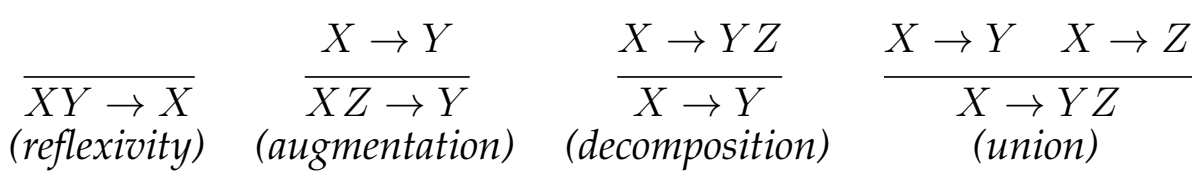

As an example consider the relation schema WORK and the NFD set $\Sigma$ that consists of Emp $\rightarrow$ Dept and Dept $\rightarrow M g r$. An application of the reflexivity axiom allows us to infer the NFD Emp $\rightarrow$ Emp. Applying the augmentation rule to Dept $\rightarrow M g r$, we can infer Emp, Dept $\rightarrow M g r$. Applying the union rule to Emp $\rightarrow$ Dept and Emp $\rightarrow$ Emp we infer Emp $\rightarrow$ Emp, Dept.

\section{Algorithms}

Again, the notion of a closure $X_{\Sigma}^{*}$ for an attribute set $X$ with respect to an NFD set $\Sigma$ is of vital interest. As was the case for FDs, the question whether an NFD set $\Sigma$ implies an NFD $X \rightarrow Y$ is the same as asking whether $Y \subseteq X_{\Sigma}^{*}[8]$. However, the closure $X_{\Sigma}^{*}$ with respect to a set of NFDs must be computed differently from that with respect to a set of FDs. Indeed, in the context of SQL relations, the mapping $(\cdot)_{\Sigma}^{*}$ is no longer a closure operation. More precisely, the operation is no longer idempotent. For example, consider the relation schema $R=$ WORK, $X=\{E m p\}$ and $\Sigma=\{E m p \rightarrow$ Dept,Dept $\rightarrow$ Mgr $\}$. Then $X_{\Sigma}^{*}=\{E m p$, Dept $\}$, but $(\text { Emp }, \text { Dept })_{\Sigma}^{*}=\{$ Emp, Dept,$M g r\}$. Therefore, $X_{\Sigma}^{*} \neq\left(X_{\Sigma}^{*}\right)_{\Sigma}^{*}$. 
The following algorithm computes the attribute set closure with respect to a set of NFDs [8]. The algorithm can be implemented such that the implication problem with input $\Sigma \cup\{X \rightarrow Y\}$ can be decided in time $\mathcal{O}(\|\Sigma \cup\{X \rightarrow Y\}\|)$.

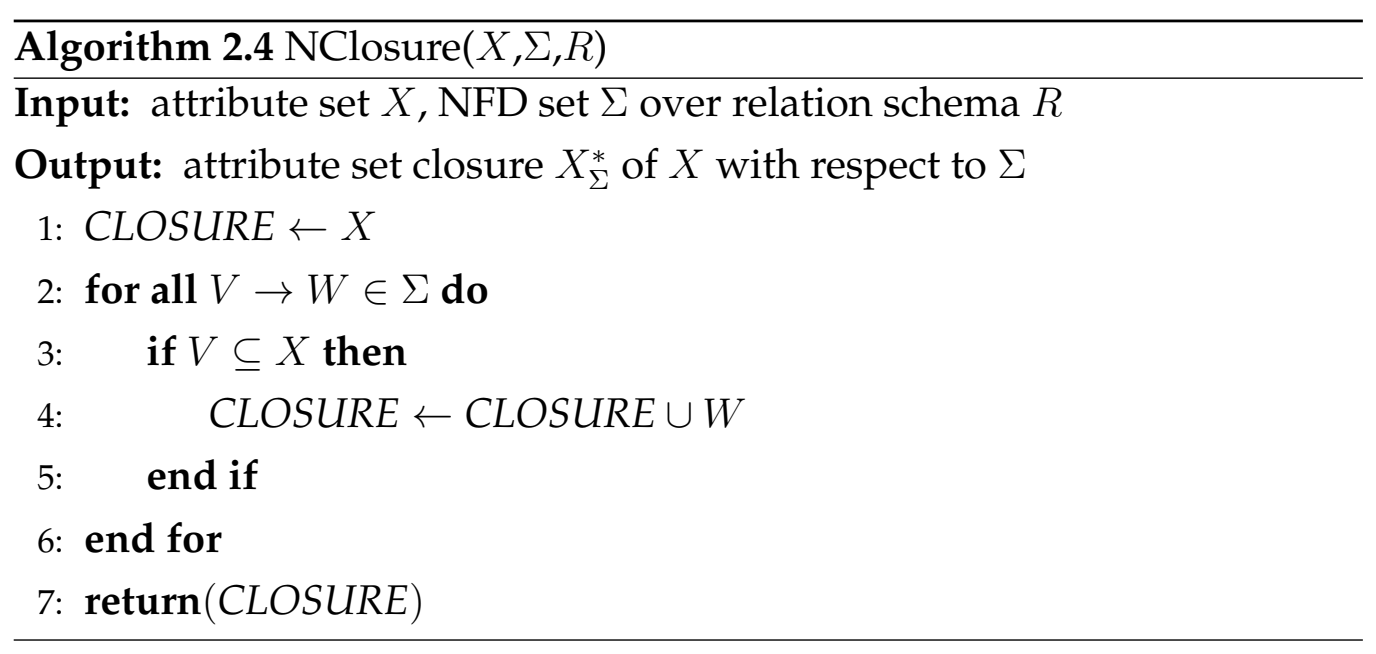

For example, on input

$$
(X=\{E m p\}, \Sigma=\{E m p \rightarrow \text { Dept }, \text { Dept } \rightarrow M g r\}, R=\text { WORK }),
$$

Algorithm 2.4 computes $X_{\Sigma}^{*}=\{$ Emp, Dept $\}$. Indeed, the only NFD $V \rightarrow$ $W \in \Sigma$ that satisfies $V \subseteq X$ is $E m p \rightarrow$ Dept.

\subsubsection{FDs and NOT NULL constraints over SQL Relations}

SQL allows the declaration of attributes as NOT NULL [33]. This means that SQL relations must be total on all attributes declared NOT NULL. This feature of SQL provides data designers and engineers with a lot of flexibility. They can decide which information is necessary to know, and which information can potentially remain partial.

Atzeni and Morfuni [8] studied the implication problem for the combined class of NFDs and NOT NULL constraints over subsumption-free SQL relations. 
Following Atzeni and Morfuni, we define a null-free subschema (NFS) over the relation schema $R$ as an expression $n f_{s}\left(R_{s}\right)$ where $R_{s} \subseteq R$. The NFS $n f s\left(R_{s}\right)$ over $R$ is satisfied by a partial relation $r$ over $R$, denoted by $\models_{r} n f s\left(R_{s}\right)$, if and only if $r$ is $R_{s}$-total.

The SQL relation $r_{S Q L}$ of Table 2.9 satisfies the NFS $n f_{S}(E m p, M g r)$ and violates the NFS $n f s$ (Emp, Dept), for example.

It suffices to fix a single null-free subschema for a given relation schema, which corresponds to the set of attributes declared NOT NULL. Note that an NFS $n f s\left(R_{s}\right)$ is implied by a set $\Sigma=\left\{n f s\left(R_{s}^{1}\right), \ldots, n f s\left(R_{s}^{n}\right)\right\}$ of NFSs if and only if $R_{s}$ is a subset of the union $R_{s}^{1} \cup \ldots \cup R_{s}^{n}$.

The NFS $n f s\left(R_{s}\right)$ has an impact on the implication of NFDs. Consider again the relation schema $R=$ WORK, the NFD set $\Sigma=\{E m p \rightarrow$ Dept,Dept $\rightarrow M g r\}$ and let $\varphi$ denote the NFD Emp $\rightarrow M g r$. Let $n f_{S}\left(R_{s}\right)$ be the NFS of $R$ where $R_{s}=\{E m p, M g r\}$. Then $\Sigma \cup\left\{n f s\left(R_{s}\right)\right\} \forall \varphi$, as the subsumption-free SQL relation of Table 2.9 shows. However, if $R_{s}=\{$ Dept $\}$, then $\Sigma \cup\left\{n f s\left(R_{s}\right)\right\} \models \varphi$ holds indeed.

The presence of an NFS $n f_{s}\left(R_{s}\right)$ subsumes both the case of total relations $\left(R_{s}=R\right)$ and the case where null markers can occur on each attribute $\left(R_{s}=\emptyset\right)$.

At this point, a convention regarding the notation is introduced. Since only a single NFS $n f_{s}\left(R_{s}\right)$ is required for each relation schema $R$, and since an NFS $n f_{s}\left(R_{s}^{\prime}\right)$ is implied by $n f_{s}\left(R_{s}\right)$ if and only if $R_{s}^{\prime} \subseteq R_{s}$, the implication problem of NFDs in the presence of an NFS is studied instead. The problem is to decide, whether for an arbitrary relation schema $R$, an arbitrary $\operatorname{NFS} n f_{s}\left(R_{s}\right)$ and an arbitrary set $\Sigma \cup\{\varphi\}$ of NFDs over $R, \Sigma \cup\left\{n f_{s}\left(R_{s}\right)\right\} \models \varphi$ holds. Here, we write $\Sigma \models{ }_{R_{s}} \varphi$ instead of $\Sigma \cup\left\{n f_{s}\left(R_{s}\right)\right\} \models \varphi$.

\section{Axiomatization}

Atzeni and Morfuni established an axiomatization for the implication problem of NFDs in the presence of an NFS [8]. 
Theorem 2.7. The following set $\mathfrak{A}$, consisting of reflexivity axiom, union rule, decomposition rule, and null transitivity rule, forms a finite axiomatization for the class of NFDs and NOT NULL constraints over subsumption-free SQL relations.

$$
\underset{\substack{X Y \rightarrow X \\
\text { (reflexivity) }}}{\frac{X \rightarrow Y \quad X \rightarrow Z}{X \rightarrow Y Z}} \quad \begin{gathered}
\frac{X \rightarrow Y Z}{X \rightarrow Y} \\
\begin{array}{c}
\text { (union) } \\
\text { (decomposition) }
\end{array}
\end{gathered} \quad \frac{X \rightarrow Y \quad Y \rightarrow Z}{\begin{array}{c}
X \rightarrow Z \\
\text { (null transitivity) }
\end{array}}
$$

Note that the null transitivity rule can only infer the NFD $X \rightarrow Z$ from the NFDs $X \rightarrow Y$ and $Y \rightarrow Z$, if all the attributes in $Y-X$ have been declared NOT NULL, i.e., are members of the NFS $n f s\left(R_{s}\right)$. Also note that the so-called augmentation rule

$$
\frac{X \rightarrow Y}{X Z \rightarrow Y}
$$

follows from the reflexivity axiom and the null transitivity rule [8].

As an example consider the relation schema WORK, the NFD set $\Sigma$ that consists of Emp $\rightarrow$ Dept and Dept $\rightarrow M g r$, and the NFS $n f_{\mathcal{S}}($ Dept $)$. An application of the null transitivity rule allows us to infer the NFD Emp $\rightarrow$ Mgr.

\section{Algorithms}

Atzeni and Morfuni also established a linear-time algorithm for deciding the implication problem for NFDs in the presence of an NFS [8]. As Beeri and Bernstein did for total relations [10], Atzeni and Morfuni utilized the notion of an attribute set closure $X_{\Sigma, R_{s}}^{*}=\left\{A \in R \mid \Sigma \models_{R_{s}} X \rightarrow A\right\}$ of an attribute set $X$ with respect to an NFD set $\Sigma$ and an NFS $n f_{s}\left(R_{s}\right)$ over the relation schema $R$. An NFD $X \rightarrow Y$ over $R$ is implied by $\Sigma$ in the presence of $n f s\left(R_{s}\right)$ if and only if $Y \subseteq X_{\Sigma, R_{s}}^{*}$ holds. Algorithm 2.5 computes the attribute set closure $X_{\Sigma, R_{s}}^{*}$ of $X$ with respect to $\Sigma$ and $n f_{s}\left(R_{s}\right)$ over $R[\overline{8}]$. For example, on input

$$
\left(X=\{E m p\}, \Sigma=\{E m p \rightarrow \text { Dept }, \text { Dept } \rightarrow M g r\}, R_{s}=\text { Dept }, R=\text { WORK }\right),
$$




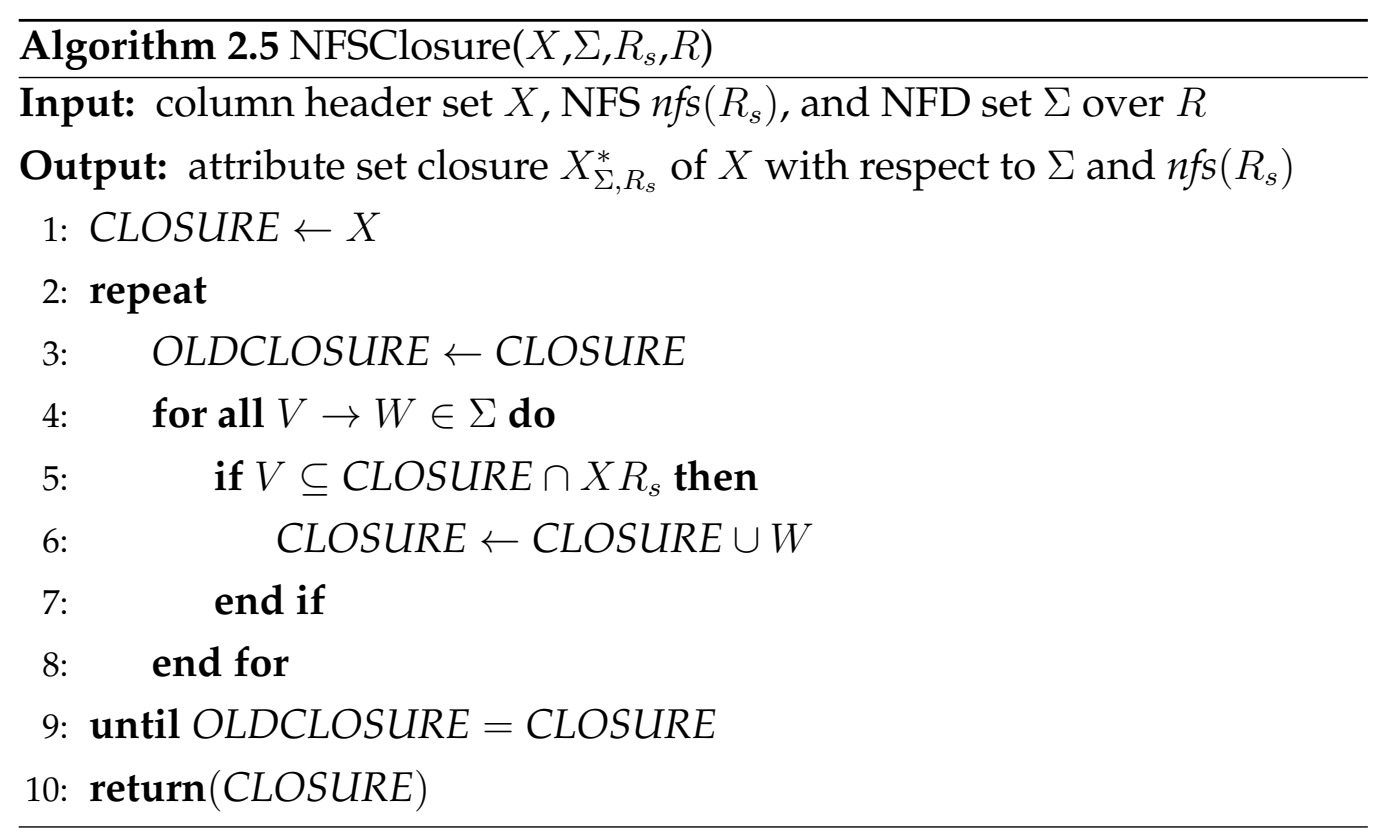

Algorithm 2.5 computes $X_{\Sigma}^{*}=\{$ Emp, Dept, Mgr $\}$.

\subsubsection{FDs over Codd Relations}

Levene and Loizou introduced and axiomatized the classes of weak and strong functional dependencies with respect to a possible world semantics [86].

A weak functional dependency (WFD) over a relation schema $R$ is an expression $\diamond(X \rightarrow Y)$ where $X, Y \subseteq R$. A Codd relation $r$ over $R$ satisfies the WFD $\diamond(X \rightarrow Y)$ if and only if there is some possible world $p \in \operatorname{Poss}(r)$ such that for all tuples $t, t^{\prime} \in p$ the following holds: if $t(X)=t^{\prime}(X)$, then $t(Y)=t^{\prime}(Y)$.

A strong functional dependency (SFD) over a relation schema $R$ is an expression $\square(X \rightarrow Y)$ where $X, Y \subseteq R$. A Codd relation $r$ over $R$ satisfies the SFD $\square(X \rightarrow Y)$ if and only if for all possible worlds $p \in \operatorname{Poss}(r)$, and for all tuples $t, t^{\prime} \in p$ the following holds: if $t(X)=t^{\prime}(X)$, then $t(Y)=$ $t^{\prime}(Y)$. 


\begin{tabular}{ccc}
\hline Emp & Dept & Mgr \\
\hline Dilbert & Information Systems & unk \\
unk & Information Systems & Gates \\
\hline
\end{tabular}

Table 2.10: WFDs and SFDs in Codd relation $r_{\text {Codd }}$

Both WFDs and SFDs occur in the real world. As an illustration consider the Codd relation $r_{\text {Codd }}$ of Table 2.10 over WORK. The relation $r_{\text {Codd }}$ satisfies:

- $\square(E m p \rightarrow D e p t)$ since every substitution of the unk occurrence in the Emp column results in a relation that satisfies the FD Emp $\rightarrow$ Dept.

- $\diamond(D e p t \rightarrow M g r)$ since there is a substitution of the unk occurrence in the Mgr column such that the FD Dept $\rightarrow$ Mgr is satisfied by the resulting possible world.

WFDs and SFDs have advantages and disadvantages over one another: SFDs can be maintained efficiently since any update of an unk occurrence to a non-null value cannot violate the corresponding FD. This, however, is not the case for WFDs. On the other hand, the weak approach to satisfaction of an FD allows a higher degree of uncertainty to be represented in the database than the strong approach (where an FD must be satisfied in all possible worlds) [86].

\section{Implication Problem and Armstrong Relations}

The axiomatization of SFDs is given by the Armstrong axioms in Theorem 2.2, while WFDs have the same axiomatization as the NFDs of Lien [89] over SQL relations, see Theorem 2.7. Algorithmic solutions to the implication problems for the class of SFDs and the class of NFDs carry over, respectively. Above all, Levene and Loizou have also established an axiomatization for the combined class of WFDs and SFDs over Codd re- 
lations [86]. However, the axiomatization is beyond the scope of this thesis. Levene and Loizou also showed that the combined class of weak and strong FDs enjoys Armstrong relations [86]. They conclude that "further research could be carried out in this area in order to generalize some of the combinatorial results concerning the size of Armstrong relations (whose size is, in general, exponential) and to construct algorithms that generate such relations". However, Koehler, Leck and Link recently established a counterexample, showing that Armstrong relations do not always exist for the combined class of weak and strong FDs [75]. In fact, already for weak and strong uniqueness constraints Armstrong relations do not always exist, and the characterization of their existence is difficult [75]. For these reasons the thesis does not discuss SFDs, but will focus on WFDs and their interaction with other constraints.

\subsubsection{Uniqueness Constraints and Keys}

This section is devoted to a brief review of previous research work on keys and uniqueness constraints in the presence of null markers.

\section{Key Sets}

Codd's principle of entity integrity states that every attribute of the primary key must be NOT NULL [31]. This principle, popular in database theory and practice, assumes that none of the attributes of the primary key may ever carry an undefined, unknown value, since otherwise one would not know what entity a tuple with an undefined value represents.

In [121], Thalheim shows that there are alternatives to Codd's principle of entity integrity. He proposes the notion of a key set, and studies its combinatorial properties. A partial relation satisfies a key set, if for each pair of distinct tuples, there is some key in the key set on which the two tuples are total and distinct. For example, the relation in Table 2.11 satisfies the key set $\{\{E m p\},\{$ Dept $\},\{M g r\}\}$, but violates the key sets $\{\{E m p\},\{D e p t\}\}$, 


\begin{tabular}{ccc}
\hline Emp & Dept & Mgr \\
\hline Dilbert & - & Gates \\
- & Information Systems & Murdoc \\
Wally & E-commerce & - \\
\hline
\end{tabular}

Table 2.11: Partial relation satisfying the key set $\{\{E m p\},\{D e p t\},\{M g r\}\}$ $\{\{E m p\},\{M g r\}\}$, and $\{\{D e p t\},\{M g r\}\}$.

\section{Codd Keys}

Candidate keys that respect Codd's principle of entity integrity were proposed and studied in [65]. Recall that the attributes in a key, that is candidate for the primary key, are supposed to be NOT NULL. For that reason an SQL relation $r$ is defined to satisfy a Codd key $\operatorname{Codd}(X)$ if and only if $r$ is $X$-total and there are not any two distinct tuples $t, t^{\prime} \in r$ such that $t(X)=t^{\prime}(X)$ holds.

The axiomatization $\mathfrak{K}$ of keys over total relations in Theorem 2.1 does not apply to Codd keys. In fact, neither the relation axiom nor the superkey rule are sound for the implication of Codd keys. For example, the SQL relation in Table 2.9 satisfies the Codd key Codd (Emp,Mgr), but it violates the Codd key Codd(Emp,Dept,Mgr).

Hartmann et al. [65] established the following simple axiomatization for Codd keys over SQL relations.

Theorem 2.8 (Hartmann et al.,2010). The following inference rule forms a finite axiomatization for the implication of Codd keys over SQL relations.

$$
\frac{\operatorname{Codd}(X) \quad \operatorname{Codd}(Y Z)}{\operatorname{Codd}(X Y)}
$$

For example, consider the set $\Sigma$ comprising the two Codd keys Codd(Emp) 
and $\operatorname{Codd}(\mathrm{Dept}, \mathrm{Mgr}$ ). An application of the key extension rule allows us to infer Codd(Emp,Mgr).

The implication problem of Codd keys can be decided in linear time in the input. Let denote $\Sigma \cup\{\operatorname{Codd}(K)\}$ be a set of Codd keys over the relation schema $R$. Algorithm 2.6 decides in time $\mathcal{O}(\| \Sigma \cup\{\operatorname{Codd}(K)\}||)$ whether $\Sigma \models \operatorname{Codd}(K)$ holds.

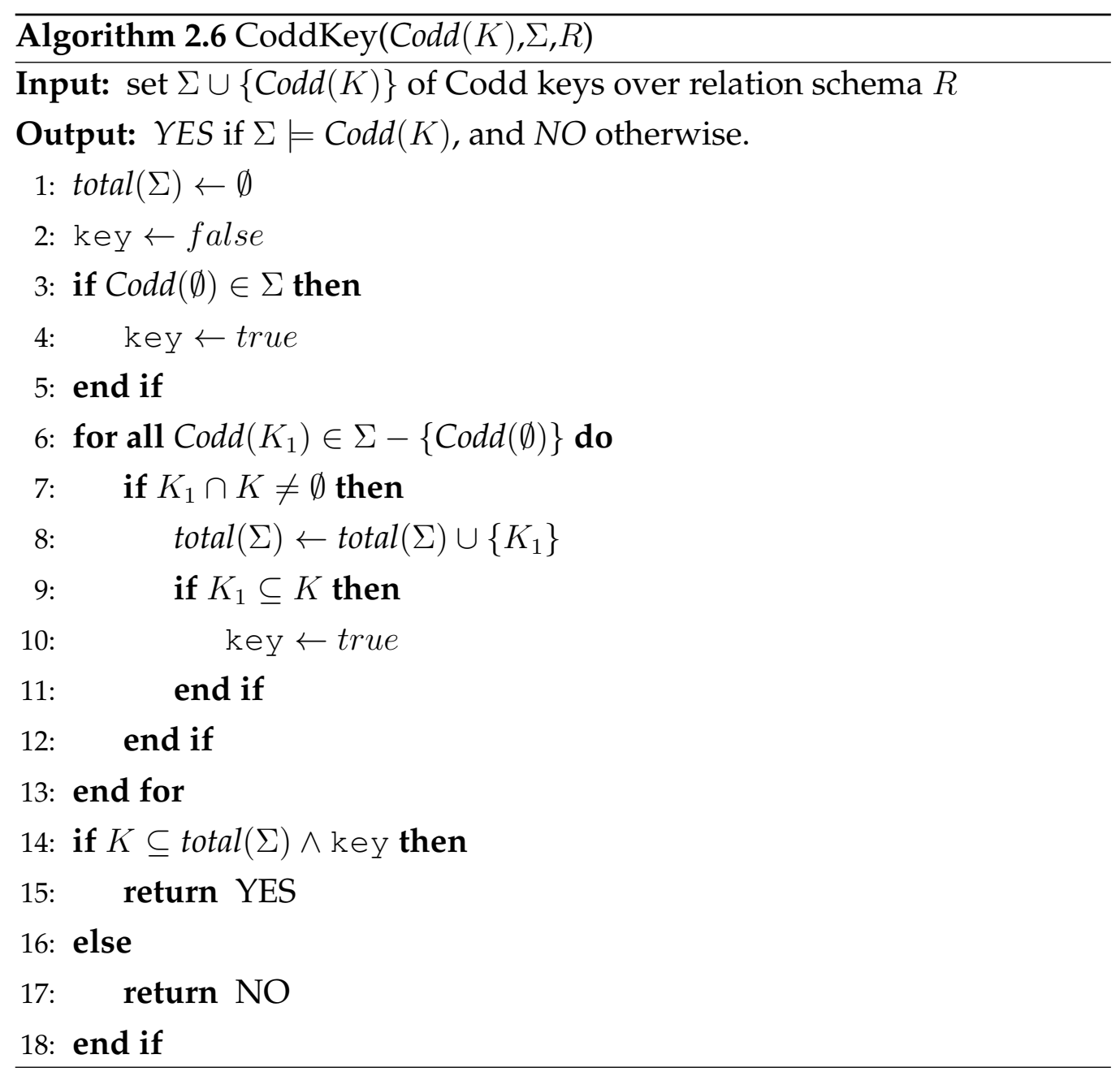

For example, consider again the set $\Sigma=\{\operatorname{Codd}(\operatorname{Emp}), \operatorname{Codd}(\operatorname{Dept}, \mathrm{Mgr})\}$ of Codd keys over relation schema WORK. Algorithm 2.6 returns NO on input (Codd (Dept), $\Sigma, R)$, but returns YES on input (Codd $(E m p, M g r), \Sigma, R)$. 


\section{Uniqueness Constraints over SQL Relations}

Surprisingly, the work on uniqueness constraints and keys over partial database instances has received little attention in the research literature. We provide some reason for why this might be the case, starting with SQL relations. Here, one could define a uniqueness constraint as follows.

A uniqueness constraint with nulls (NUC) over relation schema $R$ is an expression $u(X)$ where $X \subseteq R$. An SQL relation $r$ satisfies the NUC $u(X)$ if and only if for all tuples $t, t^{\prime} \in r$ the following holds: if $t(X)=t^{\prime}(X)$ and both $t$ and $t^{\prime}$ are $X$-total, then $t=t^{\prime}$.

The work on data dependencies has mainly been carried out under the assumption that database instances are duplicate free, i.e., instances are relations in which no two different tuples can occur that have matching values on all attributes. Under this assumption it is true for all SQL relations $r$ over a relation schema $R$, that $r$ satisfies the NUC $u(X)$ if and only if $r$ satisfies the NFD $X \rightarrow R$. Hence, uniqueness constraints occur as special cases of functional dependencies, just like their counterparts in the relational model of data.

In practice, however, duplicate elimination is expensive and not always desirable. The industry standard SQL, for example, permits occurrences of duplicate tuples in their instances, by default. SQL queries return duplicate tuples, unless specified explicitly by the DISTINCT clause. From a theoretical point of view, it therefore seems interesting to study multisets of tuples instead of sets of tuples. In what follows, we will refer to SQL tables, if we want to emphasize that we admit multisets of tuples with potential null marker occurrences of $n i$.

Interestingly, over SQL tables it is no longer true that the NUC $u(X)$ over schema $R$ holds if and only if the NFD $X \rightarrow R$ holds. More precisely, there are SQL tables $r$ that satisfy the NFD $X \rightarrow R$, but violate the NUC $u(X)$.

As an example consider the SQL table in Table 2.12 over the relation schema WORK. It satisfies the NFD Emp $\rightarrow$ WORK, but violates the NUC 


\begin{tabular}{ccc}
\hline Emp & Dept & $M g r$ \\
\hline Dilbert & $\mathrm{ni}$ & Gates \\
Dilbert & $\mathrm{ni}$ & Gates \\
\hline
\end{tabular}

Table 2.12: SQL table that violates $u(E m p)$ and satisfies Emp $\rightarrow$ Dept,Mgr

$u(E m p)$.

These explanations and example warrant a study of the implication problem for at least the combined class of NUCs and NFDs, preferably in the presence of an NFS.

\section{Uniqueness Constraints over Codd Relations}

Uniqueness constraints can be defined similarly over Codd relations. We will focus here on the weak approach.

A weak uniqueness constraint (WUC) over relation schema $R$ is an expression $\diamond \mathfrak{u}(X)$ where $X \subseteq R$. A Codd relation $r$ satisfies the WUC $\diamond \mathfrak{u}(X)$ if and only if there is a possible world $p \in \operatorname{Poss}(r)$ that satisfies the key $\operatorname{key}(X)$.

For all Codd relations $r$ over a relation schema $R$ it is true that $r$ satisfies the WUC $\diamond \mathcal{u}(X)$ if and only if $r$ satisfies the WFD $\diamond(X \rightarrow R)$. However, one may admit that possible worlds are multisets of tuples. That is, we use the term table to refer to a multiset of (total) tuples. Moreover, we use the term Codd table to refer to multisets $r$ of tuples, with potential null marker occurrences of unk, whose possible worlds include tables:

$$
\begin{aligned}
\operatorname{Poss}(r):= & \left\{r^{\prime} \mid r^{\prime} \text { is a table over } R \text { and there is a bijection } b: r \rightarrow r^{\prime}\right. \\
& \text { such that } \forall t \in r, t \text { is subsumed by } b(t) \text { and } b(t) \text { is } R \text {-total }\} .
\end{aligned}
$$

Similar to the case of NUCs and NFDs, there are Codd tables $r$ over $R$ that satisfy the WFD $\diamond(X \rightarrow R)$, but violate the WUC $\diamond \mathfrak{u}(X)$. As an example consider the Codd table in Table 2.13 over the relation schema WORK. It satisfies the WFD $\diamond(E m p \rightarrow$ WORK $)$, but violates the WUC $\diamond \mathcal{u}(E m p)$. 


\begin{tabular}{ccc}
\hline Emp & Dept & Mgr \\
\hline Dilbert & unk & Gates \\
Dilbert & Information Management & unk \\
\hline
\end{tabular}

Table 2.13: Codd table that violates $\diamond u(E m p)$ and satisfies $\diamond(E m p \rightarrow$ Dept,Mgr)

These explanations and example warrant a study of the implication problem for at least the combined class of WUCs and WFDs, preferably in the presence of an NFS.

\subsection{SQL Armstrong Tables}

The concept of Armstrong relations was summarized in Section 2.2. along with the classical theory for the class of functional dependencies, and empirical studies about the usefulness of Armstrong relations in identifying semantically meaningful functional dependencies. Section 2.4 showed how functional dependencies interact with one another over partial databases, in particular SQL and Codd tables. Since this interaction is different from that over relations, it would be highly interesting to investigate Armstrong tables. That is, Armstrong databases over SQL tables and Codd tables. In this section recent work on Armstrong databases over SQL tables is summarized. This will provide the background material necessary to understand the contributions of this thesis, including i) solutions to the implication problems for classes of uniqueness constraints and functional dependencies over Codd tables, ii) the implementation of algorithms in a graphical user interface, iii) an empirical study of the usefulness of Armstrong tables for the acquisition of semantically meaningful constraints. 


\subsubsection{NFDs Do Not Enjoy SQL Armstrong Tables}

As we have seen, in the relational model, the class of functional dependencies enjoys Armstrong relations. This includes so-called non-standard functional dependencies $\emptyset \rightarrow A$ where the left-hand side of the FD expression is an empty set of attributes.

In [64] it was shown that the class of NFDs does not enjoy Armstrong tables in the presence of a null-free subschema. For the relation schema WORK, NFD set $\Sigma=\{\emptyset \rightarrow E m p ; E m p \rightarrow$ Dept $\}$, and the NFS $n f s($ Dept,Mgr $)$ there is no Armstrong table [64].

Theorem 2.9. The class of functional dependencies with nulls in the presence of a null-free subschema does not enjoy Armstrong tables.

However, non-standard functional dependencies occur very rarely in practice and the class of standard functional dependencies with nulls does enjoy Armstrong tables in the presence of a null-free subschema. In the following, we review structural and computational properties of Armstrong tables for the combined class of NUCs, NFDs and null-free subschemata over SQL tables. Note that we implicitly assume from now on that sets of NUCs and NFDs over SQL tables do not contain any non-standard NFDs nor non-standard NUCs of the form $u(\emptyset)$.

\subsubsection{Structural Properties of SQL Armstrong Tables}

Before a structural characterization of SQL Armstrong tables can be given, some generalizations of the notions of maximal sets and agree sets are required. Indeed, the potential presence of duplicate tuples requires a new notion, too.

\section{Families of maximal sets}

Let $\Sigma$ be a set of NFDs and let $n f_{s}\left(R_{s}\right)$ be an NFS over relation schema $R$. For an attribute $C \in R$, the maximal sets $\max _{\Sigma, R_{s}}(C)$ of $C$ with respect to $\Sigma$ 
and $n f s\left(R_{s}\right)$ is defined as:

$$
\begin{aligned}
& \max _{\Sigma, R_{s}}(C):=\left\{\emptyset \neq X \subseteq R \mid \Sigma \forall_{R_{s}} X \rightarrow C \wedge\right. \\
& \left.\forall B \in R-X\left(\Sigma \models{ }_{R_{s}} X B \rightarrow C\right)\right\}
\end{aligned}
$$

The maximal sets of $R$ with respect to $\Sigma$ and $n f s\left(R_{s}\right)$ are defined as $\max _{\Sigma, R_{s}}(R)=\bigcup_{C \in R} \max _{\Sigma, R_{s}}(C)$. If $\Sigma$ and $R_{s}$ are clear from the context we may simply write $\max (C)$ and $\max (R)$, respectively.

Thus, the maximal sets of an attribute $C$ with respect to $\Sigma$ and $n f_{s}\left(R_{s}\right)$ are the maximal attribute subsets of $R$ that do not functionally determine $C$. Note that the empty set $\emptyset$ is not considered as maximal due to the exclusion of non-standard functional dependencies $\emptyset \rightarrow C$ from NFD sets. Therefore, an Armstrong table does not need to violate any non-standard NFDs.

As an example, consider the relation schema WORK with attributes Emp, Dept, and Mgr, and NFS $n f s\left(\mathrm{WORK}_{s}\right)$ where $\mathrm{WORK}_{s}=\{E m p, M g r\}$. Furthermore, let $\Sigma$ consist of the two NFDs Emp $\rightarrow$ Dept and Dept $\rightarrow M g r$. We obtain

- $\max _{\Sigma, \mathrm{WORK}_{s}}(\operatorname{Emp})=\{\{$ Dept,Mgr $\}\}$,

- $\max _{\Sigma, \mathrm{WORK}_{s}}($ Dept $)=\{\{M g r\}\}$, and

- $\max _{\Sigma, \mathrm{WORK}_{s}}(\mathrm{Mgr})=\{\{E m p\}\}$.

Specifically, $\Sigma$ does not imply the NFD Emp $\rightarrow M g r$ in the presence of WORK $_{s}=\{$ Emp, Mgr $\}$.

\section{Duplicate sets}

As seen in Equation (2.1) of Theorem 2.4 it is a necessary condition for an Armstrong relation that for each maximal set there must be distinct rows in the Armstrong relation whose strong agree set is the maximal set. This 
is to guarantee that all the NFDs not implied by the set of NUCs and NFDs in the presence of an NFS can be violated. Over tables, however, it is still possible that there are NUCs $u(X)$ not implied by $\Sigma$ in the presence of $n f s\left(R_{s}\right)$ over $R$, even if the NFD $X \rightarrow R$ is implied. For this reason, it is required for the computed Armstrong table that for all attribute sets $X$ that are maximal with this property, there must be distinct rows in the table whose strong agree set is $X$. This motivates the following definition [64].

Let $\Sigma$ be a set of standard NUCs and NFDs and let $n f_{s}\left(R_{s}\right)$ be an NFS over relation schema $R$. We define the duplicate sets $\operatorname{dup}_{\Sigma, R_{s}}(R)$ of $R$ with respect to $\Sigma$ and $n f s\left(R_{s}\right)$ as follows:

$$
\begin{aligned}
\operatorname{dup}_{\Sigma, R_{s}}(R):= & \left\{X \subseteq R \mid \Sigma \models_{R_{s}} X \rightarrow R \wedge \Sigma \not \nvdash_{R_{s}} u(X) \wedge\right. \\
& \left.\forall A \in R-X\left(\Sigma=_{R_{s}} u(X A)\right)\right\}
\end{aligned}
$$

If $\Sigma$ and $n f_{s}\left(R_{s}\right)$ are clear from the context we may simply write $d u p(R)$.

As an example, consider the relation schema WORK with attributes Emp, Dept, and Mgr, and NFS $n f s\left(\mathrm{WORK}_{s}\right)$ where $\mathrm{WORK}_{s}=\{E m p, M g r\}$. Furthermore, let $\Sigma$ consist of the two NFDs Emp $\rightarrow$ Dept and Dept $\rightarrow$ Mgr. We obtain

$$
d u p_{\Sigma, \text { WORK }_{s}}(\text { WORK })=\{\{\text { Emp, Dept, Mgr }\}\}
$$

Indeed, $\Sigma$ solely consists of NFDs and, thus, does not imply any uniqueness constraint with nulls. That is, an Armstrong table for $\Sigma$ must violate every uniqueness constraint with nulls. This can be achieved by including a pair of duplicate tuples which have matching non-null values on all the attributes in $\{$ Emp, Dept, Mgr $\}$.

\section{Strong and weak agree sets}

Equation (2.1) of Theorem 2.4 shows that the notion of agree sets is useful in characterizing Armstrong relations. For tables, which can feature oc- 


\begin{tabular}{|c|ccc|}
\hline Row & Emp & Dept & Mgr \\
\hline$t_{1}$ & Dilbert & IT & Gates \\
$t_{2}$ & Wally & IT & Gates \\
$t_{3}$ & Kim & Design & Jobs \\
$t_{4}$ & Jack & Marketing & Jobs \\
$t_{5}$ & Casey & ni & Murdoc \\
$t_{6}$ & Casey & ni & Key \\
$t_{7}$ & Snowden & Security & Nixon \\
$t_{8}$ & Snowden & Security & Nixon \\
\hline
\end{tabular}

Table 2.14: Table $r$ over WORK

currences of null markers, this notion requires further refinement. In [64] the authors defined strong and weak notions of agree sets.

Let $R$ be a relation schema, $r$ be a table over $R$, and $t_{1}, t_{2}$ be two tuples of $r$. The agree set of two tuples $t_{1}, t_{2}$ is defined as

$$
\begin{aligned}
\operatorname{ag}\left(t_{1}, t_{2}\right):= & \{(X, Y) \mid \forall A \in R( \\
& \left(\left(t_{1}(A)=t_{2}(A) \wedge t_{1}(A) \neq \mathrm{ni}\right) \leftrightarrow A \in X\right) \wedge \\
& \left.\left.\left(t_{1}(A)=t_{2}(A) \leftrightarrow A \in Y\right)\right)\right\} .
\end{aligned}
$$

The agree set of $r$ is defined as $a g(r)=\left\{a g\left(t_{1}, t_{2}\right) \mid t_{1}, t_{2} \in r \wedge t_{1} \neq t_{2}\right\}$. The strong agree set of $r$ is defined as $a g^{s}(r)=\{X \mid(X, Y) \in a g(r)\}$, and the weak agree set of $r$ is $a g^{w}(r)=\{Y \mid(X, Y) \in a g(r)\}$. Finally, for $X \in a g^{s}(r)$, we have $w(X)=\bigcap\{Y \mid(X, Y) \in \operatorname{ag}(r)\}$.

As an example, consider the SQL table $r$ over WORK in Table 2.14. Using the first letters of Emp, Dept, and Mgr, we have:

- $\operatorname{ag}(r)=\{(D M, D M),(M, M),(E, E D),(E D M, E D M),(\emptyset, \emptyset)\}$

- $a g^{s}(r)=\{D M, M, E, E D M, \emptyset\}$,

- $a g^{w}(r)=\{D M, M, E D, E D M, \emptyset\}$, and

- $w(E)=\{E D\}$. 


\section{Structural Characterization of SQL Armstrong Tables}

As shown in Theorem 2.10 [64], the notions of maximal sets, duplicate sets, strong and weak agree sets allow us to characterize Armstrong tables structurally.

Theorem 2.10. Let $R$ be a relation schema, $\Sigma$ a set of standard NUCs and NFDs, and $\operatorname{nfs}\left(R_{s}\right)$ an NFS over $R$. For all tables $r$ over $R$ it holds that $r$ is an Armstrong table for $\Sigma$ and $\operatorname{nfs}\left(R_{s}\right)$ if and only if all of the following conditions are satisfied:

1. $\forall A \in R \forall X \in \max _{\Sigma, R_{s}}(A)\left(X \in \operatorname{ag}^{s}(r) \wedge A \notin w(X)\right)$,

2. $\forall X \in a g^{s}(r)\left(X_{\Sigma, R_{s}}^{*} \subseteq w(X)\right)$

3. $\forall X \in \operatorname{dup}_{\Sigma, R_{s}}(R)\left(X \in a g^{s}(r)\right)$,

4. $\forall X \in a g^{s}(r) \forall u(Z) \in \Sigma(Z \nsubseteq X)$,

5. $R_{s}=\{A \in R \mid \forall t \in r(t(A) \neq n i)\}$.

In Theorem 2.10, the first and third conditions ensure that all NFDs and NUCs not implied by $\Sigma$ in the presence of $n f s\left(R_{s}\right)$ are violated by $r$, the second and fourth conditions ensure that all NFDs and NUCs in $\Sigma$ are satisfied by $r$, and the last condition ensures that $r$ is total on the null-free subschema and nowhere else.

\subsubsection{Computational Properties}

Theorem 2.10 has been exploited to devise an algorithm for computing tables that are Armstrong for any given set of standard NUCs and NFDs on any given relation schema [64]. Given the input, the idea is to compute the maximal set families and duplicate sets first, and then to construct a table whose agree sets realize the maximal and duplicate sets. The algorithms for these computations are summarized in the following. 


\section{Computing the maximal set families}

The computation of the maximal set families, given a relation schema $R$, an NFD set $\Sigma$ and an NFS $n f s\left(R_{s}\right)$ over R, generalizes Algorithm 2.2 which computes the maximal set families for a given FD set.

\section{Computing duplicate sets}

To compute $d u p_{\Sigma, R_{s}}(R)$ we generate the hyper-graph $\mathcal{H}=(V, E)$ with vertex set $V=R$ and the set

$$
E=\left\{K-R_{s} \mid u(K) \in \Sigma\right\}
$$

as hyper-edges. From this we obtain $d u p_{\Sigma, R_{s}}(R)$ as

$$
\operatorname{dup}_{\Sigma, R_{s}}(R)=\left\{R-X \mid X \in \operatorname{Tr}(\mathcal{H}) \wedge \forall M \in \max _{\Sigma, R_{s}}(R)(R-X \nsubseteq M)\right\}
$$

where $\operatorname{Tr}(\mathcal{H})$ denotes the minimal transversals of the hyper-graph $\mathcal{H}$ [59].

Consider again the relation schema WORK, the NFS $n f_{s}\left(\mathrm{WORK}_{s}\right)$ where WORK $_{s}=\{E m p, M g r\}$, and let $\Sigma$ consist of the two NFDs Emp $\rightarrow$ Dept and Dept $\rightarrow M g r$. The resulting hyper-graph $\mathcal{H}$ has the vertex set WORK and the hyper-edge set $E$ is empty. It follows that the only minimal transversal $X$ of $\mathcal{H}$ is the empty set, whose complement is $\{E m p, D e p t, M g r\}$. This set is not contained in any of the maximal sets with respect to $\Sigma$ and $n f s\left(\right.$ WORK $\left._{s}\right)$. Thus, $\operatorname{dup}_{\Sigma, \text { WORK }_{s}}($ WORK $)=\{\{$ Emp, Dept, Mgr $\}\}$.

\section{Armstrong table computation}

Algorithm 2.8 computes an Armstrong table for any given set $\Sigma$ of NUCs and NFDs and NFS $n f s\left(R_{s}\right)$ over any given relation schema $R[64,91]$. The idea of the algorithm is to compute the maximal sets for all attributes with respect to the standard NFD set $\Sigma[\mathrm{FD}]=\{X \rightarrow R \mid u(X) \in \Sigma\} \cup\{X \rightarrow$ $Y \mid X \rightarrow Y \in \Sigma\}$ in lines (2-4), to compute the duplicate sets in line (5), to realize all of these sets in lines (8-14), and to add null marker occurrences for all attributes that do not belong to the NFS in lines (15-22). 


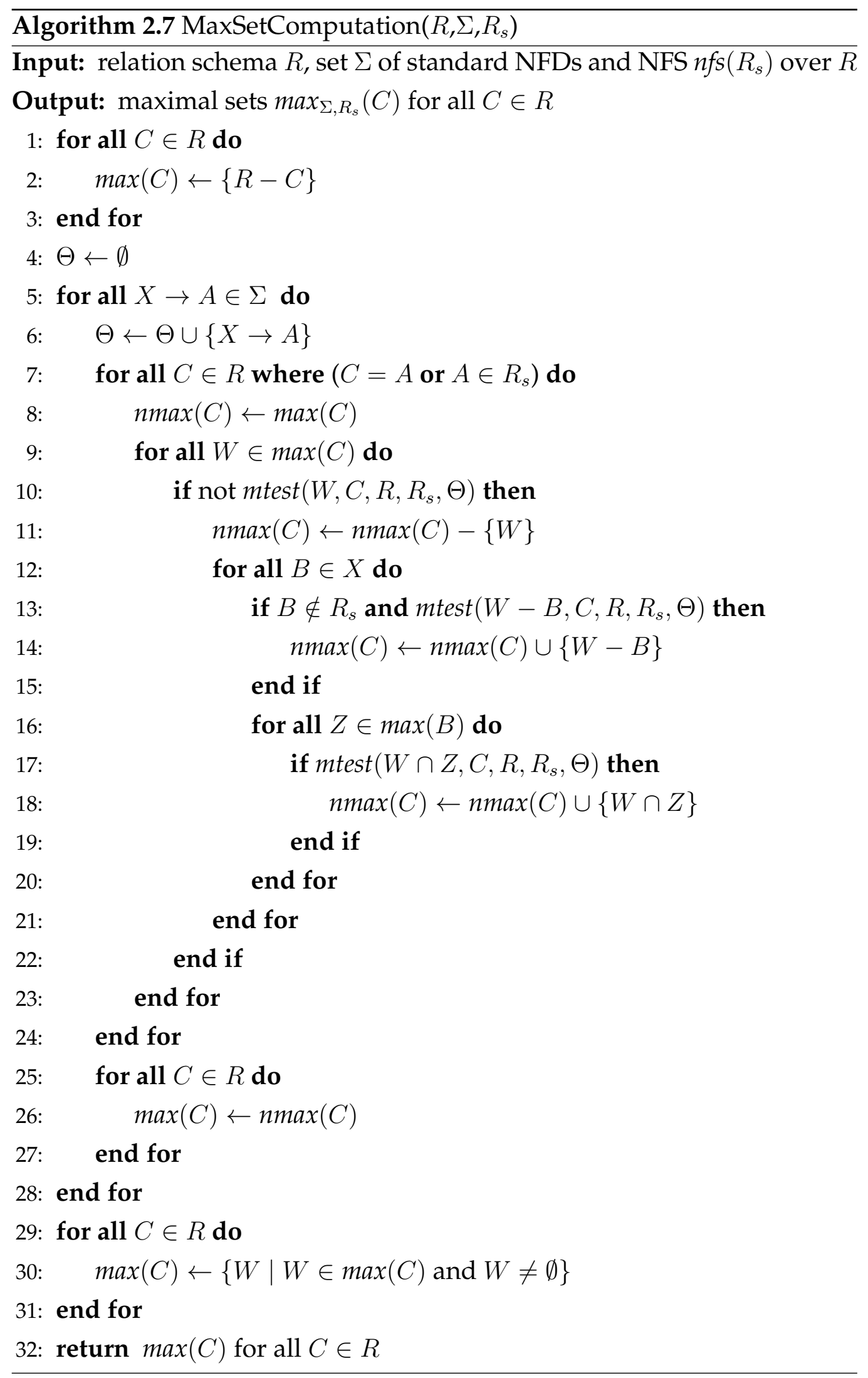




\begin{tabular}{|c|ccc|}
\hline Row & Emp & Dept & $M g r$ \\
\hline$t_{1}$ & $c_{E m p, 1}$ & $c_{\text {Dept }, 1}$ & $c_{M g r, 1}$ \\
$t_{2}$ & $c_{E m p, 2}$ & $c_{\text {Dept }, 1}$ & $c_{M g r, 1}$ \\
$t_{3}$ & $c_{E m p, 3}$ & $c_{\text {Dept }, 3}$ & $c_{M g r, 3}$ \\
$t_{4}$ & $c_{E m p, 4}$ & $c_{\text {Dept }, 4}$ & $c_{M g r, 3}$ \\
$t_{5}$ & $c_{E m p, 5}$ & $\mathrm{ni}$ & $c_{M g r, 5}$ \\
$t_{6}$ & $c_{E m p, 5}$ & $\mathrm{ni}$ & $c_{M g r, 6}$ \\
$t_{7}$ & $c_{E m p, 7}$ & $c_{\text {Dept,7}}$ & $c_{M g r, 7}$ \\
$t_{8}$ & $c_{E m p, 7}$ & $c_{\text {Dept }, 7}$ & $c_{M g r, 7}$ \\
\hline
\end{tabular}

Table 2.15: Table $r_{\text {synthetic }}$ over WORK

Specifically, when $X \in \operatorname{dup}_{\Sigma, R_{s}}(R)$, then i) $X \notin \max _{\Sigma[\mathrm{NFD}], R_{s}}(R)$, ii) $Z=$ $\left\{A \in R \mid X \in \max _{\Sigma[\mathrm{NFD}], R_{s}}(A)\right\}=\emptyset$, and iii) $R_{s} \subseteq X$. Therefore, lines (9-11) will add to the table tuples that strongly agree on the elements of $\operatorname{dup}_{\Sigma, R_{s}}(R)$.

As a continuation of our running example, consider as an input to Algorithm 2.8 the relation schema WORKS $=\{$ Emp,Dept,Mgr $\}$ with $n f s\left(\mathrm{WORK}_{s}\right)$ where $\mathrm{WORK}_{s}=\{E m p, M g r\}$, and NFD set $\Sigma$ consisting of Emp $\rightarrow$ Dept and Dept $\rightarrow$ Mgr. The corresponding output is the Armstrong table shown in Table 2.15. Suitable substitutions result in the SQL table shown in Table 2.14 .

\section{Complexity considerations}

The general worst-case exponential time complexity and rather conservative use of time carry over from Algorithm 2.3 that computes Armstrong relations [64,91].

Theorem 2.11. Let $R$ be a relation schema, $\Sigma$ be a set of standard NUCs and NFDs and $n f s\left(R_{s}\right)$ an NFS over $R$. Let $r$ be a minimum-sized Armstrong table 


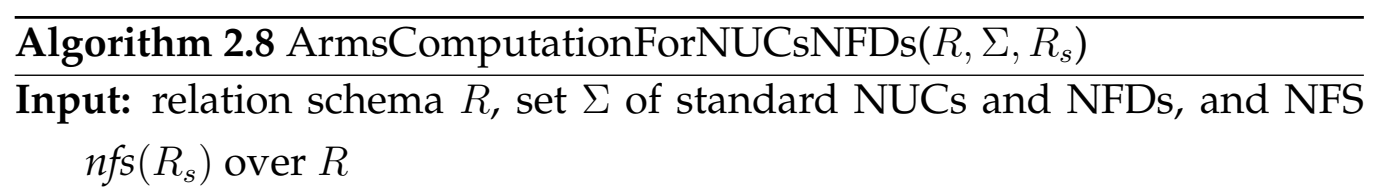

Output: Armstrong table $r$ for $\Sigma$ and $n f_{S}\left(R_{s}\right)$

1: Let $c_{A, 1}, c_{A, 2}, \ldots \in \operatorname{dom}(A)$ be distinct

2: for all $A \in R$ do

3: $\quad$ Compute $\max _{\Sigma[\mathrm{NFD}], R_{s}}(A)$ using Algorithm 2.7

4: end for

5: Compute $d u p_{\Sigma, R_{s}}(R)$

6: $r \leftarrow \emptyset$

7: $i \leftarrow 1$

8: for all $X \in \max _{\Sigma[\mathrm{NFD}], R_{s}}(R) \cup \operatorname{dup}_{\Sigma[\mathrm{NFD}], R_{s}}(R)$ do

9: $\quad Z \leftarrow\left\{A \in R \mid X \in \max _{\Sigma[F D], R_{s}}(A)\right\}$

10: $\quad r \leftarrow r \cup\left\{t_{i}, t_{i+1}\right\}$ where $\forall A \in R$

11: $\quad t_{i}(A) \leftarrow\left\{\begin{array}{l}c_{A, i}, \text { if } A \in X Z R_{s} \\ \mathrm{ni}, \text { else }\end{array}\right.$ and

12:

$$
t_{i+1}(A) \leftarrow \begin{cases}c_{A, i} & , \text { if } A \in X \\ c_{A, i+1} & \text { if } A \in Z\left(R_{s}-X\right) \\ \mathrm{ni} & \text {, else }\end{cases}
$$

13: $\quad i \leftarrow i+2$

14: end for

15: $\operatorname{total}(r) \leftarrow\{A \in R \mid \forall t \in r(t(A) \neq \mathrm{ni})\}$

16: if $\operatorname{total}(r)-R_{s} \neq \emptyset$ then

17: $\quad$ return $r \leftarrow r \cup\left\{t_{i}\right\}$ where $\forall A \in R$

$18:$

$$
t_{i}(A) \leftarrow\left\{\begin{array}{l}
\mathrm{ni}, \text { if } A \in \operatorname{total}(r)-R_{s} \\
c_{A, i}, \text { else }
\end{array}\right.
$$

19: else

20: return $r$

21: end if 
for $\sum$ and $n f_{s}\left(R_{s}\right)$. Then

$$
\underline{\sqrt{1+8 \cdot\left|\max _{\Sigma[N F D], R_{s}}\left(R_{s}\right) \cup d u p_{\Sigma, R_{s}}(R)\right|}} \leq|r|
$$

and

$$
|r| \leq 2 \cdot\left|\max _{\Sigma[N F D], R_{s}}\left(R_{s}\right) \cup \operatorname{dup}_{\Sigma, R_{s}}\right|+1
$$

For input $\left(R, \Sigma, R_{s}\right)$, Algorithm 2.8 computes an Armstrong table for $\Sigma$ and $n f s\left(R_{s}\right)$ whose size is at most quadratic in the size of a minimum-sized Armstrong table for $\Sigma$ and $n f s\left(R_{s}\right)$.

Further it is known that there are standard NFD sets $\Sigma$ of size $\mathcal{O}(n)$ where the size of a minimum Armstrong table for $\Sigma$ is in $\mathcal{O}\left(2^{n}\right)$. Vice versa, there are standard NFD sets $\Sigma$ which have Armstrong tables for $\Sigma$ where the number of tuples is in $\mathcal{O}(n)$ and the best representation of $\Sigma$ is of size in $\mathcal{O}\left(2^{n}\right)$. It is therefore advisable to utilize both representations, the abstract constraint set $\Sigma$ and an Armstrong table for $\Sigma$ [64, 77]. Intuitively, constraint sets help identify constraints incorrectly perceived as meaningful, and Armstrong tables help identify constraints incorrectly perceived as meaningless. However, it is one of the objectives of this thesis to provide evidence for this intuition.

\subsubsection{Further Remarks}

Similar results to those in this section have recently been obtained for the combined class of cardinality constraints with upper bounds and NFDs in the presence of an NFS [63,91]. This class, however, is beyond the scope of this thesis.

The general class of NUCs and NFDs in the presence of an NFS leads naturally to the question of what structural and computational properties Armstrong tables for the individual class of NUCs and the individual class of NFDs have. Answers to these questions can be found in [64,84]. 


\subsection{Summary and Research Gap}

The final section of this chapter gives a brief summary of the literature review, identifies gaps in the research literature, and states the objectives to be addressed in this thesis.

\subsubsection{Summary}

The theory of data dependencies has mostly been addressed in the relational model of data. Here, the implication problem of functional dependencies has been studied extensively. These studies include axiomatic, algorithmic, and logical solutions as well as results on the structural and computational properties of Armstrong relations. There is also empirical evidence that Armstrong relations are useful for the discovery of semantically meaningful functional dependencies. Some attempts have been made to encompass partial information in database instances. The most popular approach in these attempts is the permission of null marker occurrences in database instances. SQL relations allow occurrences of the null marker no information, and Codd relations allow occurrences of the null marker value unknown at present. For subsumption-free SQL relations, the implication problem of functional dependencies has been characterized axiomatically, algorithmically and logically in the presence of NOT NULL constraints. For Codd relations, the implication problem has been characterized axiomatically and algorithmically for the class of weak, the class of strong, and the combined class of weak and strong functional dependencies. SQL and Codd tables, respectively, permit occurrences of the corresponding null marker as well as duplicate tuples. Previous work on constraints has not addressed Codd tables at all, while characterizations of the implication problem for a more general class of constraints, involving multivalued dependencies [55, 70, 92], and properties of Armstrong tables for the combined class of uniqueness and NOT NULL constraints as well as functional dependencies have been addressed for SQL tables. 


\subsubsection{Research gap}

As the literature review shows there is a great disparity between the state of the art in database theory and database practice. Real-world data features occurrences of duplicate tuples and subsumed tuples, and of null markers in columns declared NULL. However, over such data the class of functional dependencies does not include the class of uniqueness constraints. What is therefore missing is a foundation for constraints on tables. In particular, axiomatic, algorithmic, and logical characterizations of the implication problem for the combined class of uniqueness constraints, NOT NULL constraints and functional dependencies is missing over Codd tables, and has only been addressed for a more general class of constraints in the context of SQL tables. The constraints are essential for the design of real-world databases, and have profound applications in almost all data processing tasks. It is therefore a fundamental problem to assist database designers and data engineers in their task of discovering those constraints that are semantically meaningful for their domain of interest. For this purpose, Armstrong databases would be of great help. While recent research has focused on properties of Armstrong databases over SQL tables, there is no research regarding Armstrong databases for uniqueness constraints, NOT NULL constraints or functional dependencies over Codd tables. Prototypes that exist for Armstrong relations have not been developed yet for Armstrong databases over SQL nor Codd tables. Research on this topic could therefore result in tools which with design teams and engineers can effectively visualise, test, validate, and consolidate their understanding of the semantics of the application domain. Evidence that such tools are indeed effective for the identification of semantically meaningful constraints is therefore also missing. 


\subsubsection{Objectives}

The overall goal of this thesis is to establish a toolbox of algorithms that data engineers can utilize to communicate and consolidate their understanding about the semantics of the given application domain. The hypothesis is that this understanding can then be encoded in constraints that enforce the semantics of the application domain within the database. Three distinct areas of investigation are pursued to address this goal.

1. Foundations: The implication problem of classes of popular constraints over tables must be analyzed in depth. Here, axiomatic and algorithmic characterizations are required for the combined class of uniqueness constraints and functional dependencies in the presence of NOT NULL constraints. These insights will be used to study structural and computational properties of Armstrong databases for this combined class. For these objectives the thesis will mainly focus on these constraints in the context of Codd tables.

2. Implementation: The algorithms developed to compute Armstrong databases must be implemented in a prototype. This prototype must have an interface that enables data engineers to select their interpretation of a null marker, to effectively input their current application domain and their perception about the meaningful constraints, and to execute the Armstrong database computation. They must also be given the ability to replace the artificial values by real domain values of their choice. This can be done either prior to the computation or thereafter. For these objectives the thesis will address various classes of constraints over SQL relations, SQL tables, Codd relations, and Codd tables.

3. Evaluation: Empirical evidence will be sought to confirm that the inspection of the Armstrong databases, produced by the prototype, really are useful for the acquisition of semantically meaningful constraints. For this purpose, quantitative and qualitative measures will 
be defined. These measures can be used to give a precise definition of the term useful. Experiments with design teams will then be conducted to apply the measures. For these objectives the thesis will focus on the combined class of uniqueness constraints with nulls and functional dependencies with nulls over SQL tables with a null-free subschema. 


\section{Chapter 3}

\section{Foundations - Part I: \\ Reasoning about Constraints over Codd Tables}

In this chapter we will start to address the first objective stated at the end of Chapter 2. More specifically, we will investigate the implication problem for the combined class of weak uniqueness constraints and weak functional dependencies over Codd tables with NOT NULL constraints. In Section 3.1 we give preliminary definitions on the data model. An axiomatic characterization of the implication problem is established in Section 3.2. Subsequently in Section 3.3. the axioms are exploited to derive an algorithm for deciding the implication problem in time linear in the input size. In Section 3.4 an equivalence of the implication problem is established to that for Horn clauses in Cadoli and Schaerf's well-known $\mathcal{S}$-3 logics. Further comments on related literature are given in Section 3.5. A summary of the chapter is presented in Section 3.6. The results of this chapter have been announced in [56]. 


\subsection{Preliminary Definitions}

Codd's original proposal [30] to handle partial information suggested to add to the sets of possible domain values the unmarked null marker unk, whose meaning is "value unknown at present". Following Codd's proposal, some researchers argue that partial information is represented in SQL by using unk as a distinguished null maker [34].

Let $\mathfrak{A}=\left\{A_{1}, A_{2}, \ldots\right\}$ be a countably infinite set of symbols, called attributes. A relation schema is a finite non-empty subset $R$ of $\mathfrak{A}$. Each attribute $A$ of a relation schema $R$ is associated with an infinite domain $\operatorname{dom}(A)$ of the possible values that can occur in column $A$. To encompass partial information every attribute may have a null marker, denoted by unk $\in \operatorname{dom}(A)$. The intention of unk is to mean "value unknown at present".

For attribute sets $X$ and $Y$ we may write $X Y$ for $X \cup Y$. If $X=$ $\left\{A_{1}, \ldots, A_{m}\right\}$, then we may write $A_{1} \cdots A_{m}$ for $X$. In particular, we may write simply $A$ to represent the singleton $\{A\}$. A tuple over $R$ (R-tuple or simply tuple, if $R$ is understood) is a function $t: R \rightarrow \bigcup_{A \in R} \operatorname{dom}(A)$ with $t(A) \in \operatorname{dom}(A)$ for all $A \in R$. The null marker occurrence $t(A)=$ unk associated with an attribute $A$ in a tuple $t$ means that the value $t(A)$ is unknown at present. For $X \subseteq R$ let $t(X)$ denote the restriction of the tuple $t$ over $R$ to $X$. A Codd table $r$ over $R$ is a finite multiset of tuples over $r$. Let $t_{1}$ and $t_{2}$ be two tuples over $R$. It is said that $t_{1}$ subsumes $t_{2}$ if for every attribute $A \in R, t_{1}(A)=t_{2}(A)$ or $t_{2}(A)=$ unk holds.

For a tuple $t$ over $R$ and a set $X \subseteq R, t$ is said to be $X$-total if for all $A \in X, t(A) \neq$ unk. Similarly, a Codd table $r$ over $R$ is said to be $X$-total, if every tuple $t$ of $r$ is $X$-total. A Codd table $r$ over $R$ is said to be a total Codd table if it is $R$-total.

Extending ideas by Levene and Loizou [86] the set of all possible worlds 
relative to a Codd table $r$ over $R$, denoted by $\operatorname{Poss}(r)$, is defined by

$\operatorname{Poss}(r):=\left\{r^{\prime} \mid r^{\prime}\right.$ is a total Codd table over $R$ and there is a bijection $b: r \rightarrow r^{\prime}$ such that $\forall t \in r, t$ is subsumed by $\left.b(t)\right\}$.

This definition of possible worlds embodies the closed world assumption (CWA) [72], since only $R$-total tuples from the Codd table $r$ can be present in $\operatorname{Poss}(r)$.

A weak uniqueness constraint (WUC) over a relation schema $R$ is a statement of the form $\diamond \mathcal{u}(X)$, where $X \subseteq R$. A Codd table $r$ over $R$ is said to satisfy the WUC $\diamond \mathfrak{u}(X)$ over $R$, if there is some $r^{\prime} \in \operatorname{Poss}(r)$ such that for all $t_{1}, t_{2} \in r^{\prime}$, if $r_{1} \neq r_{2}$, then $t_{1}(X) \neq t_{2}(X)$. The satisfaction of a WUC in a Codd table reduces to the satisfaction of a key when the Codd table is $R$-total. In this case there is exactly one $r^{\prime} \in \operatorname{Poss}(r)$ and $\forall r^{\prime} \in \operatorname{Poss}(r)$ is equivalent to $\exists r^{\prime} \in \operatorname{Poss}(r)$.

A weak functional dependency (WFD) over a relation schema $R$ is a statement of the form $\diamond(X \rightarrow Y)$, where $X Y \subseteq R$. A Codd table $r$ over $R$ is said to satisfy the WFD $\diamond(X \rightarrow Y)$ over $R$, if there is some $r^{\prime} \in \operatorname{Poss}(r)$ such that for all $t_{1}, t_{2} \in r^{\prime}$, if $t_{1}(X)=t_{2}(X)$, then $t_{1}(Y)=t_{2}(Y)$. We note that the definition of satisfaction of a WFD in a Codd table reduces to the standard definition of the satisfaction of a functional dependency when the Codd table is $R$-total.

The weak approach to satisfaction of a functional dependency by a Codd table allows a higher degree of uncertainty to be represented in the database than the strong approach (where a functional dependency must be satisfied in all possible worlds) [86]. The disadvantage of the weak over the strong approach is that strongly satisfied functional dependencies are easier to maintain [86]. Hence, both approaches complement one another. It is future work to combine strong and weak functional dependencies over Codd tables, in particular in combination with NOT NULL constraints.

Following Atzeni and Morfuni [8] a null-free subschema (NFS) over the relation schema $R$ is an expression $n f s\left(R_{s}\right)$ where $R_{s} \subseteq T$. The NFS $n f s\left(R_{s}\right)$ 
over $R$ is satisfied by a Codd table $r$ over $R$, denoted by $\models_{r} n f s\left(R_{s}\right)$, if and only if $r$ is $R_{s}$-total. SQL allows the specification of attributes as NOT NULL. NFSs occur in everyday database practice: the set of attributes declared NOT NULL forms an NFS over the underlying relation schema.

We introduce an extension of the notion of agree sets of distinct rows to the presence of null markers [11,96]. For two tuples $t_{1}, t_{2}$ over relation schema $R$ we define

$$
\begin{aligned}
& a g^{s}\left(t_{1}, t_{2}\right)=\left\{A \in R \mid t_{1}(A)=t_{2}(A) \text { and } t_{1}(A) \neq \text { unk } \neq t_{2}(A)\right\} \\
& a g^{w}\left(t_{1}, t_{2}\right)=\left\{A \in R \mid t_{1}(A)=\text { unk or } t_{2}(A)=\text { unk }\right\} \\
& a g\left(t_{1}, t_{2}\right)=a g^{s}\left(t_{1}, t_{2}\right) \cup a g^{w}\left(t_{1}, t_{2}\right)
\end{aligned}
$$

Intuitively, this definition makes perfect sense: i) two tuples strongly agree on an attribute if they agree in all possible worlds, and ii) two tuples weakly agree on an attribute if there is a possible world on which they agree on the attribute. Moreover, for a Codd table $r$ over relation schema $R$ we define

$$
\begin{aligned}
& a g^{s}(r)=\left\{a g^{s}\left(t_{1}, t_{2}\right) \mid t_{1}, t_{2} \in r \text { and } t_{1} \neq t_{2}\right\}, \\
& a g^{w}(r)=\left\{a g^{w}\left(t_{1}, t_{2}\right) \mid t_{1}, t_{2} \in r \text { and } t_{1} \neq t_{2}\right\}, \\
& a g(r)=\left\{a g\left(t_{1}, t_{2}\right) \mid t_{1}, t_{2} \in r \text { and } t_{1} \neq t_{2}\right\}
\end{aligned}
$$

That is, the agree sets of a Codd table consist of the agree sets of the pairs of distinct tuples they contain. Next we establish a syntactic characterization of the satisfaction of WUCs and WFDs.

Proposition 3.1. Let $X Y \subseteq R$ and $r$ be a Codd table over relation schema $R$. Then

1. $r$ satisfies $\diamond \mathcal{u}(X)$ if and only if for all $t_{1}, t_{2} \in r$, if $X \subseteq a g^{s}\left(t_{1}, t_{2}\right)$, then $t_{1}=t_{2}$.

2. $r$ satisfies $\diamond(X \rightarrow Y)$ if and only if for all $t_{1}, t_{2} \in r$, if $X \subseteq a g^{s}\left(t_{1}, t_{2}\right)$, then $Y \subseteq a g\left(t_{1}, t_{2}\right)$. 
Proof. We show first that $r$ satisfies $\diamond \mathcal{u}(X)$ if and only if for all $t_{1}, t_{2} \in r$, if $t_{1} \neq t_{2}$, then $X \nsubseteq a g^{s}\left(t_{1}, t_{2}\right)$.

If there are some $t_{1}, t_{2} \in r$ such that $t_{1} \neq t_{2}$ and $X \subseteq a g^{s}\left(t_{1}, t_{2}\right)$, then every possible world of $r$ violates the key $\operatorname{key}(X)$. Vice versa, if for every different $t_{1}, t_{2} \in r$ there is some $A \in X$ such that i) $t_{1}(A) \neq t_{2}(A)$ or ii) $t_{1}(A)=$ unk or iii) $t_{2}(A)=$ unk holds, then there is a possible world of $r$ that satisfies the key $k e y(X)$. Such a possible world can be obtained from $r$ by replacing the occurrences of unk by unique domain values.

It remains to show that $r$ satisfies $\diamond(X \rightarrow Y)$ if and only if for all $t_{1}, t_{2} \in$ $r$, if $X \subseteq a g^{s}\left(t_{1}, t_{2}\right)$, then $Y \subseteq a g\left(t_{1}, t_{2}\right)$.

If there are some $t_{1}, t_{2} \in r$ such that $X \subseteq a g^{s}\left(t_{1}, t_{2}\right)$ and $Y \nsubseteq a g\left(t_{1}, t_{2}\right)$, then there is some $A \in Y$ such that unk $\neq t_{1}(A) \neq t_{2}(A) \neq$ unk. Consequently, every possible world of $r$ violates the FD $X \rightarrow Y$. Vice versa, if for every $t_{1}, t_{2} \in r$, strong agreement of $t_{1}$ and $t_{2}$ on $X$ implies agreement of $t_{1}$ and $t_{2}$ on $Y$, then there is a possible world of $r$ that satisfies the FD $X \rightarrow Y$. Such a possible world can be obtained from $r$ by replacing the occurrences of unk by appropriate domain values.

Example 3.1. Consider the relation schema WORK with attributes Emp, Dept and Mgr. Let $r$ denote the following Codd table over WORK:

\begin{tabular}{|c|c|c|}
\hline Emp & Dept & $M g r$ \\
\hline Dilbert & IT & Gates \\
Dilbert & unk & Murdoc \\
\hline
\end{tabular}

The following two tables are possible worlds of $r$ in $\operatorname{Poss}(r)$.

\begin{tabular}{|c|c|c|}
\hline Emp & Dept & $M g r$ \\
\hline Dilbert & IT & Gates \\
Dilbert & IT & Murdoc \\
\hline
\end{tabular}

\begin{tabular}{|c|c|c|}
\hline Emp & Dept & Mgr \\
\hline Dilbert & IT & Gates \\
Dilbert & Security & Murdoc \\
\hline
\end{tabular}

Based on the semantics of WUCs and WFDs or based on Proposition 3.1 it is simple to observe that $r$ satisfies $\diamond \mathcal{u}(M g r), \diamond \mathcal{u}($ Emp, Dept $), \diamond(E m p \rightarrow$ Dept $)$ and $\diamond(D e p t \rightarrow M g r)$. However, $r$ violates $\diamond u(E m p)$ and $\diamond(E m p \rightarrow M g r)$. 
In schema design and maintenance data dependencies are normally specified as semantic constraints on the Codd tables intended to be instances of the schema. During the design process or the lifetime of a database one usually needs to determine further dependencies which are implied by the given ones. Let $R$ be a relation schema, let $n f_{s}\left(R_{s}\right)$ denote an NFS over $R$, and let $\Sigma \cup\{\varphi\}$ be a set of WUCs and WFDs over $R$. We say that $\Sigma$ implies $\varphi$ in the presence of $n f_{s}\left(R_{s}\right)$, denoted by $\Sigma \models_{R_{s}} \varphi$, if every Codd table $r$ over $R$ that satisfies $\Sigma$ and $n f s\left(R_{s}\right)$ also satisfies $\varphi$. If $\Sigma$ does not imply $\varphi$ in the presence of $n f_{s}\left(R_{s}\right)$ we may also write $\Sigma \forall_{R_{s}} \varphi$. Let $\Sigma_{R_{s}}^{*}=\left\{\varphi \mid \Sigma \models_{R_{s}} \varphi\right\}$ be the semantic closure of $\Sigma$ in the presence of $n f s\left(R_{s}\right)$. One can attempt to determine the semantic closure by a syntactic approach, e.g. by applying the inference rules from Table 3.1 below. These inference rules have the form

$$
\frac{\text { premise }}{\text { conclusion }} \text { condition, }
$$

and inference rules without any premises are called axioms. An inference rule is called sound for the implication of WUCs and WFDs in the presence of an NFS, if whenever the elements in the premise of the rule and the NFS are satisfied by some Codd table and the elements and NFS satisfy the conditions of the rule, then the Codd table also satisfies the element in the conclusion of the rule. For a finite set $\Sigma \cup\{\varphi\}$ of WUCs and WFDs and a set $\mathfrak{R}$ of inference rules let $\Sigma \vdash_{\mathfrak{R}} \varphi$ denote the inference of $\varphi$ from $\Sigma$ by $\mathfrak{R}$. That is, there is some sequence $\gamma=\left[\sigma_{1}, \ldots, \sigma_{n}\right]$ of WUCs and WFDs such that $\sigma_{n}=\varphi$ and every $\sigma_{i}$ is an element of $\Sigma$ or results from an application of an inference rule in $\Re$ to some elements in $\left\{\sigma_{1}, \ldots, \sigma_{i-1}\right\}$. For a finite set $\Sigma$ of WUCs and WFDs, let $\Sigma_{\mathfrak{R}}^{+}=\left\{\varphi \mid \Sigma \vdash_{\mathfrak{R}} \varphi\right\}$ be its syntactic closure under inferences by $\mathfrak{R}$. A set $\mathfrak{R}$ of inference rules is said to be sound (complete) for the implication of WUCs and WFDs in the presence of an NFS if for every relation schema $R$, for every NFS $n f s\left(R_{s}\right)$ over $R$ and for every set $\Sigma$ of WUCs and WFDs over $R$ we have $\Sigma_{\mathfrak{R}}^{+} \subseteq \Sigma_{R_{s}}^{*}\left(\Sigma_{R_{s}}^{*} \subseteq \Sigma_{\mathfrak{R}}^{+}\right)$. The (finite) set $\mathfrak{R}$ is said to be a (finite) axiomatization for the implication of WUCs and 
WFDs in the presence of an NFS if $\Re$ is both sound and complete.

As a final remark of this section it is pointed out that we defined Codd tables to be finite database instances. Naturally, this has led us to define the finite implication problem for the classes of constraints under inspection. In theory, one may also permit Codd tables with an infinite number of rows which would lead us to the unrestricted implication problem. However, for the classes of WUCs and WFDs over Codd tables with an NFS, their associated finite and unrestricted implication problems coincide. Of course, if there is no unrestricted Codd table that satisfies $\Sigma$ and $n f_{s}\left(R_{s}\right)$ and violates $\varphi$, then there is in particular no finite Codd table with that property. Vice versa, if there is some finite Codd table that satisfies $\Sigma$ and $n f_{s}\left(R_{s}\right)$ and violates $\varphi$, then there must be two tuples in that table that already offend $\varphi$ while satisfying $\Sigma$ and $n f s\left(R_{s}\right)$.

Further Outline. In the remainder of this chapter we establish a comprehensive analysis of the implication problem for WUCs and WFDs in the presence of an NFS. First, we characterize the implication problem by a finite ground axiomatization. Next, we develop an algorithm that decides the implication problem in time linear in the input. Finally, we establish an equivalence to the implication of goal and definite clauses in Cadoli and Schaerf's well-known family of $\mathcal{S}$-3 logics.

\subsection{Axiomatic Characterization}

We begin our analysis of the implication problem for WUCs and WFDs over Codd tables with an NFS by an axiomatic characterization. This axiomatization forms the basis for our future analysis, extends the wellknown theory of keys and functional dependencies over relations, and the existing work on weak and strong functional dependencies over Codd relations, see Chapter 2

Let $\mathfrak{S}$ denote the set of inference rules in Table 3.1. Reflexivity axiom, decomposition, null transitivity, and union rule are the familiar rules from 


\begin{tabular}{|cc|}
\hline $\begin{array}{c}\diamond(X Y \rightarrow X) \\
(\text { reflexivity) }\end{array}$ & $\frac{\diamond(X \rightarrow Y Z)}{\diamond(X \rightarrow Y)}$ \\
$\frac{\diamond(X \rightarrow Y) \diamond(Y \rightarrow Z)}{\text { (decomposition) }}$ \\
$\begin{array}{c}\diamond(X \rightarrow Z) \\
(\text { null transitivity) }\end{array}$ & $\frac{\diamond(X \rightarrow Y) \diamond(X \rightarrow Z)}{\left(X R_{s}\right.}$ \\
$\frac{\diamond u(X)}{\diamond(X \rightarrow Y)}$ & $\begin{array}{c}\text { (union) } \\
(\text { demotion })\end{array}$ \\
\hline
\end{tabular}

Table 3.1: Axiomatization $\mathfrak{S}$ of Weak Uniqueness Constraints and Weak Functional Dependencies over Codd Tables with a Null-free Subschema $n f s\left(R_{s}\right)$. 
Atzeni and Morfuni's axiomatization of NFDs over SQL relations [8, 70]. The demotion rule says that every Codd table that satisfies a WUC $\diamond \mathcal{u}(X)$ also satisfies any WFD with left-handed side $X$. Furthermore, the null pullback rule captures further non-trivial interactions of WUCs and WFDs over Codd tables with an NFS. We show first that these rules are sound, exploiting Proposition 3.1

Lemma 3.1. The inference rules in $\mathfrak{S}$ are sound for the implication of WUCs and WFDs over Codd tables with NFSs.

Proof. We exhibit the soundness for each of the six rules, exploiting the syntactic characterization of the semantics of WUCs and WFDs from Proposition 3.1 .

For the reflexivity axiom let $r$ be an arbitrary Codd table over $R$, and $\diamond(X Y \rightarrow X)$ a WFD over $R$. Trivially, any two tuples $t_{1}, t_{2} \in r$ that satisfy $X Y \subseteq a g^{s}\left(t_{1}, t_{2}\right)$ also satisfy $X \subseteq a g\left(t_{1}, t_{2}\right)$ since $X \subseteq X Y \subseteq a g^{s}\left(t_{1}, t_{2}\right) \subseteq$ $a g\left(t_{1}, t_{2}\right)$. Hence, by Proposition 3.1. $r$ satisfies $\diamond(X Y \rightarrow X)$.

For the decomposition rule let $r$ be an arbitrary Codd table over $R$ such that $r$ violates the WFD $\diamond(X \rightarrow Y)$ over $R$. Hence, by Proposition 3.1. there are two tuples $t_{1}, t_{2} \in r$ that satisfy $X \subseteq a g^{s}\left(t_{1}, t_{2}\right)$ but $Y \nsubseteq a g\left(t_{1}, t_{2}\right)$. Consequently, $t_{1}, t_{2} \in r$ satisfy $X \subseteq a g^{s}\left(t_{1}, t_{2}\right)$ but also not $Y Z \nsubseteq a g\left(t_{1}, t_{2}\right)$. Thus, by Proposition 3.1. $r$ also violates the WFD $\diamond(X \rightarrow Y Z)$ over $R$.

For the null transitivity rule let $r$ be an arbitrary Codd table over $R$ such that $r$ violates the WFD $\diamond(X \rightarrow Z)$ over $R$, satisfies the WFD $\diamond(X \rightarrow Y)$ over $R$ and satisfies the condition $Y \subseteq X R_{s}$ where $n f s\left(R_{s}\right)$ is an NFS over $R$. Hence, by Proposition 3.1, there are two tuples $t_{1}, t_{2} \in r$ that satisfy $X \subseteq a g^{s}\left(t_{1}, t_{2}\right)$ but $Z \nsubseteq a g\left(t_{1}, t_{2}\right)$. Since $r$ satisfies the WFD $\diamond(X \rightarrow Y)$, Proposition 3.1 tells us that $Y \subseteq a g\left(t_{1}, t_{2}\right)$. However, since $r$ satisfies $Y \subseteq$ $X R_{s}$, it even follows that $Y \subseteq a g^{s}\left(t_{1}, t_{2}\right)$. Since $Z \nsubseteq a g\left(t_{1}, t_{2}\right)$, we conclude by Proposition 3.1 that $r$ violates the WFD $\diamond(Y \rightarrow Z)$.

For the union rule let $r$ be an arbitrary Codd table over $R$ such that $r$ violates the WFD $\diamond(X \rightarrow Y Z)$ over $R$. Hence, by Proposition 3.1, there are two tuples $t_{1}, t_{2} \in r$ that satisfy $X \subseteq a g^{s}\left(t_{1}, t_{2}\right)$ but $Y Z \nsubseteq a g\left(t_{1}, t_{2}\right)$. 
Consequently, $Y \nsubseteq a g\left(t_{1}, t_{2}\right)$ or $Z \nsubseteq a g\left(t_{1}, t_{2}\right)$. Again by Proposition 3.1. $r$ violates the WFD $\diamond(X \rightarrow Y)$ or the WFD $\diamond(X \rightarrow Z)$.

For the demotion rule let $r$ be an arbitrary Codd table over $R$ such that $r$ violates the WFD $\diamond(X \rightarrow Y)$ over $R$. Hence, by Proposition 3.1, there are two tuples $t_{1}, t_{2} \in r$ that satisfy $X \subseteq a g^{s}\left(t_{1}, t_{2}\right)$ but $Y \nsubseteq a g\left(t_{1}, t_{2}\right)$. The latter condition implies that $t_{1} \neq t_{2}$. Again by Proposition 3.1, $r$ violates the WUC $\diamond u(X)$.

For the null pullback rule let $r$ be an arbitrary Codd table over $R$ such that $r$ violates the WFD $\diamond u(X)$ over $R$, satisfies the WFD $\diamond(X \rightarrow Y)$ over $R$ and satisfies the condition $Y \subseteq X R_{s}$ where $n f s\left(R_{s}\right)$ is an NFS over $R$. Hence, by Proposition 3.1, there are two different tuples $t_{1}, t_{2} \in r$ that satisfy $X \subseteq a g^{s}\left(t_{1}, t_{2}\right)$. Since $r$ satisfies the WFD $\diamond(X \rightarrow Y)$, Proposition 3.1 tells us that $Y \subseteq a g\left(t_{1}, t_{2}\right)$. However, since $r$ satisfies $Y \subseteq X R_{s}$, it even follows that $Y \subseteq a g^{s}\left(t_{1}, t_{2}\right)$. We conclude by Proposition 3.1 that $r$ violates the WUC $\diamond u(Y)$.

Let $\mathfrak{F}$ consist of the reflexivity axiom, the decomposition, the null transitivity, and the union rule from Table 3.1. We use a classical proof to establish the completeness of $\mathfrak{F}$ for the implication of WFDs over Codd tables with an NFS.

Theorem 3.1. The set $\mathfrak{F}$ is a finite axiomatization for the implication of WFDs over Codd tables with an NFS.

Proof. Let $R$ be a relation schema, $\Sigma$ a set of WFDs and $n f s\left(R_{s}\right)$ an NFS over $R$. Let $\diamond(X \rightarrow Y) \notin \Sigma_{\mathfrak{F}}^{+}$. We establish the completeness of $\mathfrak{F}$ by showing that $\diamond(X \rightarrow Y) \notin \Sigma_{R_{s}}^{*}$. That is, we construct a Codd table $r$ over $R$ that is $R_{s}$-total, satisfies $\Sigma$ and violates $\diamond(X \rightarrow Y)$. Let $X_{\mathfrak{F}}^{+}=\left\{A \in R \mid \Sigma \vdash_{\mathfrak{F}}\right.$ $\diamond(X \rightarrow A)\}$. Consequently, $Y \nsubseteq X_{\mathfrak{F}}^{+}$and there is some $A_{0} \in Y-X_{\mathfrak{F}}^{+}$. Indeed, if $Y \subseteq X_{\mathfrak{F}}^{+}$, then $\diamond(X \rightarrow Y) \in \Sigma_{\mathfrak{F}}^{+}$by the union rule. Let $r:=\left\{t_{1}, t_{2}\right\}$ 
be the Codd table over $R$ where $t_{1}, t_{2}$ are defined by:

\begin{tabular}{ccc}
\hline$X_{\mathfrak{F}}^{+} \cap X R_{s}$ & $\left(R_{s}-X_{\mathfrak{F}}^{+}\right) A_{0}$ & Otherwise \\
\hline $0 \cdots 0$ & $0 \cdots 0$ & $0 \cdots 0$ \\
$0 \cdots 0$ & $1 \cdots 1$ & unk $\cdots$ unk
\end{tabular}.

Proposition 3.1 shows that $r$ violates the WFD $\diamond(X \rightarrow Y)$ since $A_{0} \in Y$. Furthermore, $r$ is $R_{s}$-total. It remains to show that $r$ satisfies $\Sigma$. Let $\diamond(U \rightarrow$ $V) \in \Sigma$ and $U \subseteq a g^{s}\left(t_{1}, t_{2}\right)$. By construction, $U \subseteq X_{\mathfrak{F}}^{+}$. Hence, $\diamond(X \rightarrow U) \in$ $\Sigma_{\mathfrak{F}}^{+}$holds by the definition of $X_{\mathfrak{F}}^{+}$. Furthermore, by construction, $U \subseteq X R_{s}$. From $\diamond(X \rightarrow U) \in \Sigma_{\mathfrak{F}}^{+}, \diamond(U \rightarrow V) \in \Sigma$, and $U \subseteq X R_{s}$, it follows by the null transitivity rule that $\diamond(X \rightarrow V) \in \Sigma_{\mathfrak{F}}^{+}$. Thus, $V \subseteq X_{\mathfrak{F}}^{+}$. In particular, $A_{0} \notin V$ since otherwise $A_{0} \in X_{\mathfrak{F}}^{+}$by the decomposition rule. This means that $V \subseteq a g\left(t_{1}, t_{2}\right)$. By Proposition 3.1, $r$ satisfies $\diamond(U \rightarrow V) \in \Sigma$.

Next we establish the completeness of $\mathfrak{S}$ for the implication of the combined class of WUCs and WFDs over Codd tables with NFS by a reduction to the completeness of $\mathfrak{F}$. Two preparatory lemmata are required first.

For a set $\Sigma$ of WUCs and WFDs over relation schema $R$ let

$$
\Sigma[\mathrm{WFD}]=\{\diamond(X \rightarrow R) \mid \diamond \mathcal{u}(X) \in \Sigma\} \cup\{\diamond(X \rightarrow Y) \mid \diamond(X \rightarrow Y) \in \Sigma\} .
$$

Lemma 3.2. Let $\Sigma \cup\{\diamond(X \rightarrow Y)\}$ be a set of WUCs and WFDs, and $n f s\left(R_{s}\right)$ an NFS over relation schema $R$. Then $\Sigma \models_{R_{s}} \diamond(X \rightarrow Y)$ if and only if $\Sigma[W F D] \models_{R_{s}}$ $\diamond(X \rightarrow Y)$.

Proof. If $\Sigma[\mathrm{WFD}] \models_{R_{s}} \diamond(X \rightarrow Y)$, then $\Sigma \models_{R_{s}} \diamond(X \rightarrow Y)$ because the demotion rule is sound.

If $\Sigma[\mathrm{WFD}] \not \nvdash_{R_{s}} \diamond(X \rightarrow Y)$, then consider the two-tuple relation $r$ from the completeness proof of Theorem 3.1. In particular, $r$ satisfies $\Sigma[\mathrm{WFD}]$, $r$ is $R_{s}$-total and $r$ violates $\diamond(X \rightarrow Y)$. Moreover, $r$ satisfies every WUC $\diamond \mathcal{u}(Z) \in \Sigma$ : otherwise, the two tuples $t_{1}, t_{2} \in r$ would be duplicates as $\diamond(Z \rightarrow R) \in \Sigma[$ WFD] is satisfied by $r$, and $r$ would also satisfy $\diamond(X \rightarrow Y)$, a contradiction. Consequently, $r$ shows that $\Sigma \forall_{R_{s}} \diamond(X \rightarrow Y)$. 
Lemma 3.3. Let $\Sigma \cup\{\diamond u(X)\}$ be a set of WUCs and WFDs, and $n f s\left(R_{s}\right)$ an NFS over relation schema $R$. Then $\Sigma \models_{R_{s}} \diamond \mathcal{U}(X)$ if and only if $\Sigma[W F D] \models_{R_{s}} \diamond(X \rightarrow$ $R$ ) and there is some $\diamond u(Z) \in \Sigma$ such that $Z \subseteq X R_{s}$.

Proof. Suppose $\Sigma[\mathrm{WFD}] \models_{R_{s}} \diamond(X \rightarrow R)$ and there is some $\diamond u(Z) \in \Sigma$ such that $Z \subseteq X R_{s}$. The soundness of the decomposition rule shows that $\Sigma[\mathrm{WFD}] \models_{R_{s}} \diamond(X \rightarrow Z)$. The soundness of the null pullback rule shows that $\Sigma[\mathrm{WFD}] \models_{R_{s}} \diamond \mathcal{u}(X)$. Finally, the soundness of the demotion rule shows that $\Sigma \models R_{s} \diamond \mathfrak{u}(X)$.

Suppose that $\Sigma \models_{R_{s}} \diamond \mathcal{u}(X)$. The soundness of the demotion rule means that $\Sigma \models_{R_{s}} \diamond(X \rightarrow R)$ holds, too. From Lemma 3.2 we conclude that $\Sigma[\mathrm{WFD}] \models_{R_{s}} \diamond(X \rightarrow R)$ holds. It remains to show that if $\Sigma \models_{R_{s}} \diamond \mathfrak{u}(X)$ holds, then there is some $\diamond u(Z) \in \Sigma$ such that $Z \subseteq X R_{s}$. We show the contraposition: if $Z \nsubseteq X R_{s}$ holds for all $\diamond u(Z) \in \Sigma$, then $\Sigma \forall_{R_{s}} \diamond u(X)$. Indeed, let $r^{\prime}$ be the Codd table consisting of the following two tuples:

\begin{tabular}{cc}
\hline$X R_{s}$ & $R-\left(X R_{s}\right)$ \\
\hline $0 \cdots 0$ & $0 \cdots 0$ \\
$0 \cdots 0$ & unk $\cdots$ unk \\
\hline
\end{tabular}

Trivially, $r^{\prime}$ satisfies every WFD and is $R_{s}$-total. For any $\diamond \mathfrak{u}(Z) \in \Sigma$ we know that $Z \nsubseteq X R_{s}$ holds. Hence, $r^{\prime}$ also satisfies every WUC in $\Sigma$, and thereby, $\Sigma$ itself. Since $r^{\prime}$ violates $\diamond \mathcal{u}(X)$, it follows that $\Sigma \nvdash_{R_{s}} \diamond \mathcal{u}(X)$.

Theorem 3.2. The set $\mathfrak{S}$ is a finite axiomatization for the implication of WUCS and WFDs in the presence of an NFS.

Proof. Let $\Sigma \cup\{\varphi\}$ be a set of WUCs and WFDs, and $n f_{s}\left(R_{s}\right)$ an NFS over relation schema $R$. We exploit Lemmata 3.2 and 3.3 and Theorem 3.1 to show directly that $\Sigma \models_{R_{s}} \varphi$ entails $\Sigma \vdash_{\mathfrak{S}} \varphi$ holds.

Suppose that $\varphi$ denotes the WFD $\diamond(X \rightarrow Y)$. From Lemma 3.2 we conclude that $\Sigma[\mathrm{WFD}] \models_{R_{s}} \diamond(X \rightarrow Y)$ holds. As $\mathfrak{F}$ is complete for the implication of WFDs over Codd tables with NFS, it follows that $\Sigma\left[\right.$ WFD] $\vdash_{\mathfrak{F}}$ $\diamond(X \rightarrow Y)$ holds. Since $\mathfrak{F} \subseteq \mathfrak{S}$ we conclude further that $\Sigma[\mathrm{WFD}] \vdash_{\mathfrak{S}} \diamond(X \rightarrow$ 
$Y)$ holds. Finally, the demotion rule guarantees that $\Sigma \vdash_{\mathfrak{S}} \sigma$ holds for all $\sigma \in \Sigma[\mathrm{WFD}]$. Hence, $\Sigma \vdash_{\mathfrak{S}} \diamond(X \rightarrow Y)$.

Suppose now that $\varphi$ denotes the WUC $\diamond \mathfrak{u}(X)$. From Lemma 3.3 we conclude that $\Sigma[\mathrm{WFD}] \models_{R_{s}} \diamond(X \rightarrow R)$ holds, and that there is some $\diamond \mathcal{u}(Z) \in \Sigma$ where $Z \subseteq X R_{s}$ holds. As in the previous case, it follows that $\Sigma \vdash_{\mathfrak{S}} \diamond(X \rightarrow R)$ holds, and the decomposition rule shows $\Sigma \vdash_{\mathfrak{S}} \diamond(X \rightarrow Z)$. An application of the null pullback rule infers that $\Sigma \vdash_{\mathfrak{S}} \diamond \mathfrak{u}(X)$. This concludes the proof.

We write $\Sigma=_{2, R_{s}} \varphi$ to say that $\varphi$ is implied by $\Sigma$ and $n f s\left(R_{s}\right)$ over twotuple Codd tables. That is, $\Sigma \models_{2, R_{s}} \varphi$ denotes an instance of the implication problem in the world of two-tuple Codd tables: every two-tuple Codd table $r$ over $R$ that is $R_{s}$-total and satisfies every element of $\Sigma$ also satisfies $\varphi$. It is a consequence of our previous arguments that $\Sigma \models_{2, R_{s}} \varphi$ if and only if $\Sigma \models_{R_{s}} \varphi$ holds. This is an interesting model-theoretic property of our class of constraints, which will prove to be a key argument when we establish a logical characterization of their associated implication problem.

Corollary 3.1. For all relation schemata $R$, for all sets $\Sigma \cup\{\varphi\}$ of WUCs and WFDs over $R$ and for all NFSs $n f_{s}\left(R_{s}\right)$ over $R, \Sigma \models_{R_{s}} \varphi$ if and only if $\Sigma \models_{2, R_{s}} \varphi$.

Proof. Obviously, if there is some two-tuple Codd table $r$ over $R$ that is $R_{s}$-total, satisfies every element of $\Sigma$, and violates $\varphi$, then $\Sigma \nvdash_{R_{s}} \varphi$. Vice versa, if $\Sigma \nvdash_{R_{s}} \varphi$, then the proofs of Theorem 3.1 and Lemma 3.3 show how to construct a two-tuple Codd table $r$ over $R$ that is $R_{s}$-total, satisfies every element of $\Sigma$, and violates $\varphi$.

Example 3.2. Consider the relation schema WORK with attributes Emp, Dept and $M g r$, NFS WORK ${ }_{s}=\{$ Dept,$M g r\}$, and

$$
\Sigma=\{\diamond(E m p \rightarrow \text { Dept }), \diamond(D e p t \rightarrow M g r), \diamond u(M g r)\} .
$$

Then $\Sigma \models_{\text {WORK }_{s}} \diamond u(E m p)$. Indeed, one may apply the null transitivity rule to the two WFDs of $\Sigma$ to infer the WFD $\diamond(E m p \rightarrow M g r)$. One may then 
apply the null pullback rule to this WFD and the WUC from $\Sigma$ to infer the WUC $\diamond u(E m p)$.

Example 3.3. Consider the relation schema WORK with attributes Emp, Dept and $M g r$, NFS WORK $_{s}=\{$ Dept, Mgr $\}$, and

$$
\Sigma=\{\diamond(E m p \rightarrow \text { Dept }), \diamond(\text { Dept } \rightarrow \text { Mgr })\}
$$

Then $\Sigma \not \forall_{\text {WORK }_{s}} \diamond \mathcal{u}(E m p)$, as the Codd table

\begin{tabular}{ccc}
\hline Emp & Dept & $M g r$ \\
\hline Dilbert & IT & Gates \\
Dilbert & IT & Gates \\
\hline
\end{tabular}

over WORK with NFS $n f_{s}\left(\mathrm{WORK}_{s}\right)$ shows. Note that this Codd table follows the construction in the proof of Lemma 3.3 .

Example 3.4. Consider the relation schema WORK with attributes Emp, Dept and Mgr, NFS WORK ${ }_{s}=\{$ Dept $\}$, and

$$
\Sigma=\{\diamond(E m p \rightarrow \text { Dept }), \diamond(D e p t \rightarrow M g r), \diamond u(M g r)\}
$$

Then $\left.\Sigma \not\right|_{\text {WORK }_{s}} \diamond \mathcal{u}(E m p)$, as the Codd table

\begin{tabular}{ccc}
\hline Emp & Dept & $M g r$ \\
\hline Dilbert & IT & Gates \\
Dilbert & IT & unk \\
\hline
\end{tabular}

over WORK with NFS $n f_{s}\left(\mathrm{WORK}_{s}\right)$ shows. Note that this Codd table follows the construction in the proof of Theorem 3.1 .

\subsection{Algorithmic Characterization}

Given a relation schema $R$, an NFS $n f s\left(R_{s}\right)$ and a set $\Sigma$ of WUCs and WFDs over $R$, the inference system $\mathfrak{S}$ determines the semantic closure $\Sigma_{R_{s}}^{*}$. This 
usually takes time exponential in the size of the input. In practice, however, knowledge about all elements of the semantic closure is not always required. Instead, it often suffices to know whether some fixed WUC or WFD $\varphi$ is an element of $\Sigma_{R_{s}}^{*}$. In this case one must decide the instance $\Sigma \models_{R_{s}} \varphi$ of the associated implication problem. For that purpose the computation of $\Sigma_{R_{s}}^{*}$ is too expensive. In this section, we develop an algorithm that decides the implication problem efficiently.

Recall the completeness proof of Theorem 3.1 where we defined the attribute closure

$$
X_{\mathfrak{F}}^{+}:=\left\{A \in R \mid \Sigma \vdash_{\mathfrak{F}} \diamond(X \rightarrow A)\right\}
$$

for an attribute set $X$, and a set $\Sigma$ of WFDs over relation schema $R$ with NFS $n f_{\mathcal{S}}\left(R_{s}\right)$. Note that Theorem 3.1 guarantees that $X_{\mathfrak{F}}^{+}=X_{\Sigma, R_{s}}^{*}$ where

$$
X_{\Sigma, R_{s}}^{*}:=\left\{A \in R \mid \Sigma \models_{R_{s}} \diamond(X \rightarrow A)\right\} .
$$

Lemmata 3.2 and 3.3 allow us to reduce the implication problem for the combined class of WUCs and WFDs over Codd tables with NFS to the computation of attribute closures with respect to WFDs and an NFS.

Corollary 3.2. Let $\Sigma$ be a set of WUCs and WFDs over relation schema $R$ with $\operatorname{NFS} n f s\left(R_{s}\right)$. Then the following holds:

1. $\Sigma \models_{R_{s}} \diamond(X \rightarrow Y)$ if and only if $Y \subseteq X_{\Sigma[W F D], R_{s}}^{*}$ and

2. $\Sigma \models_{R_{s}} \diamond \mathcal{u}(X)$ if and only if $X_{\Sigma[W F D], R_{s}}^{*}=R$ and there is some $\diamond \mathcal{u}(Z) \in \Sigma$ such that $Z \subseteq X R_{s}$.

Corollary 3.2 motivates an algorithm that computes the attribute closure $X_{\Sigma[W F D], R_{s}}^{*}$ from a given attribute set $X$ with respect to a given set $\Sigma\left[\right.$ WFD] of WFDs over a given relation schema $R$ with given $\operatorname{NFS} n f s\left(R_{s}\right)$. Such an algorithm is shown as Algorithm 3.1 .

We show first that Algorithm 3.1 works correctly, that is, computes indeed the closure of the given attribute set with respect to the given set 


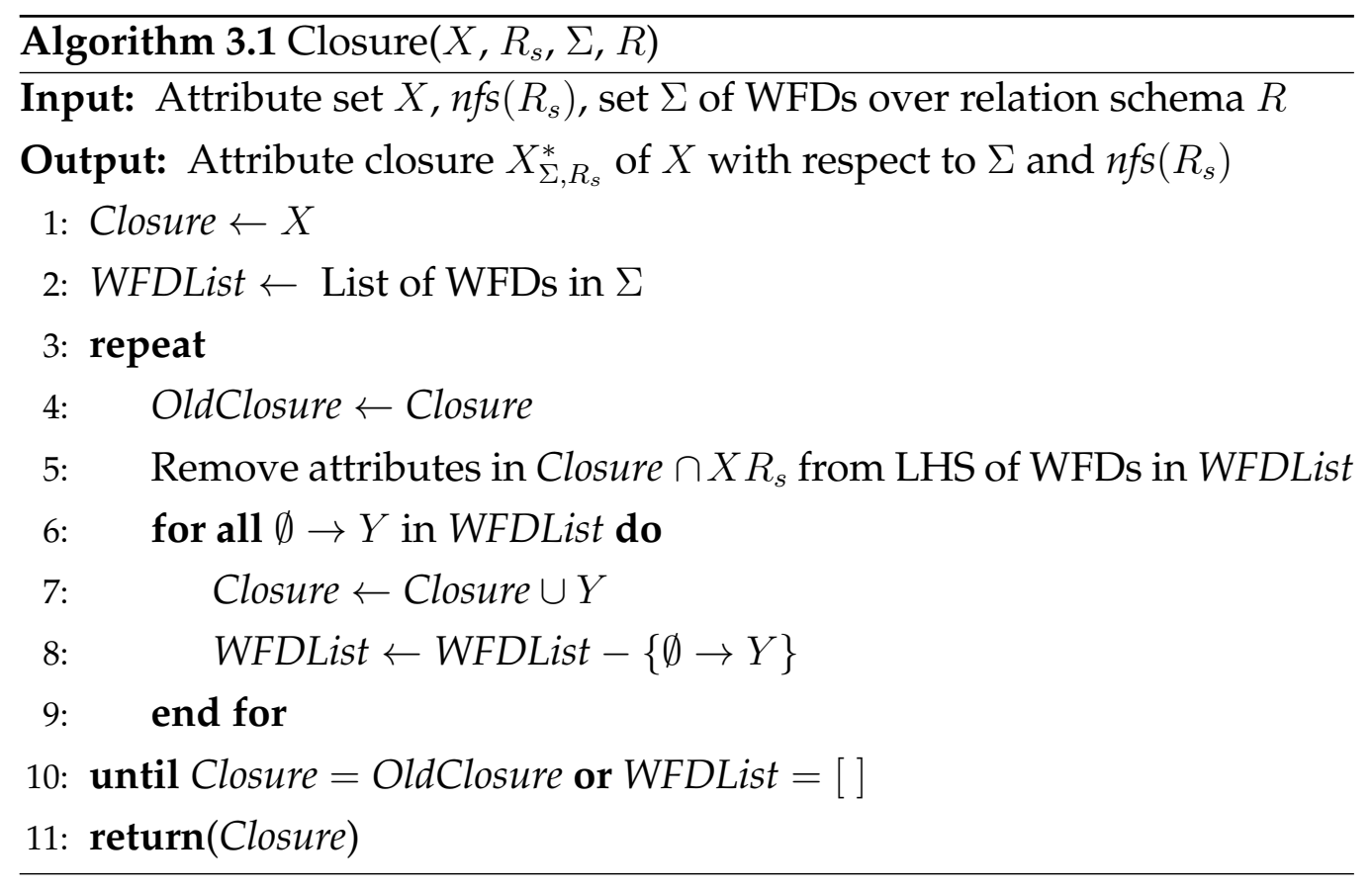

of WFDs and NFS. Note that the proof relies on the completeness of the axiomatization $\mathfrak{F}$.

Theorem 3.3. On input $\left(X, R_{s}, \Sigma, R\right)$, Algorithm 3.1 computes the attribute closure $X_{\Sigma, R_{s}}^{*}$ of $X$ with respect to the WFD set $\Sigma$ over relation schema $R$ with $\operatorname{NFS} n f s\left(R_{s}\right)$.

Proof. Let Closure denote the output of Algorithm 3.1. It needs to be shown that Closure $=X_{\Sigma, R_{s}}^{*}$. Since $X_{\Sigma, R_{s}}^{*}=X_{\mathfrak{F}}^{+}$, this is the same as showing that Closure $=X_{\mathfrak{F}}^{+}$holds. For that purpose we proceed in two stages, first showing that Closure $\subseteq X_{\mathfrak{F}}^{+}$.

We proceed by induction on the number $j$ of runs through the repeat loop between lines 3-10 of Algorithm 3.1. If $j=0$, then line 1 set Closure to $X$ and we have $X \subseteq X_{\mathfrak{F}}^{+}$due to the reflexivity axiom. Let $j>0$. The hypothesis tells us that after $j$ runs we have Closure $\subseteq X_{\mathfrak{F}}^{+}$. Consider now the $j+1$ st run which adds all attributes of $Y$ to Closure whenever there is some WFD $\diamond(Z \rightarrow Y) \in \Sigma$ where $Z \subseteq$ Closure and $Z \subseteq X R_{s}$. From 
$\diamond(X \rightarrow$ Closure $) \in \Sigma_{\mathfrak{F}}^{+}$and $Z \subseteq$ Closure we obtain $\diamond(X \rightarrow Z) \in \Sigma_{\mathfrak{F}}^{+}$by the decomposition rule. From $\diamond(X \rightarrow Z) \in \Sigma_{\mathfrak{F}}^{+}, \diamond(Z \rightarrow Y) \in \Sigma$ and $Z \subseteq X R_{s}$ we conclude $\diamond(X \rightarrow Y) \in \Sigma_{\mathfrak{F}}^{+}$by an application of the null transitivity rule. That is, also after the $j+1$ st run we have Closure $\subseteq X_{\mathfrak{F}}^{+}$.

It remains to show that $X_{\mathfrak{F}}^{+} \subseteq$ Closure holds as well. For this purpose, consider the chain

$$
\Sigma=\Sigma_{0} \subset \Sigma_{1} \subset \ldots \subset \Sigma_{k}=\Sigma_{\mathfrak{F}}^{+}
$$

where $\Sigma_{j}$ results from $\Sigma_{j-1}$ by application of a single inference rule from $\mathfrak{F}$, for $j=1, \ldots, k$. We will use induction on $j$ to show the following

$$
\text { if } \diamond(Z \rightarrow Y) \in \Sigma_{j} \text { and } Z \subseteq \text { Closure } \cap X R_{s} \text {, then } Y \subseteq \text { Closure. }
$$

Then we conclude for $j=k$ that $Y \subseteq$ Closure follows from $\diamond(Z \rightarrow Y) \in \Sigma_{\mathfrak{F}}^{+}$ and $Z \subseteq$ Closure $\cap X R_{s}$. Hence, $X_{\mathfrak{F}}^{+} \subseteq$ Closure follows for $Z=X$ and $Y=X_{\mathfrak{F}}^{+}$.

We proceed by induction on $j$. If $j=0$, then $\diamond(Z \rightarrow Y) \in \Sigma$. If $Z \subseteq$ Closure $\cap X R_{s}$, then $\emptyset \rightarrow Y$ is in WFDList due to line 5, and $Y$ will be added to Closure in line 7. Hence, $Y \subseteq$ Closure. If $j>0$, then the WFD $\diamond(Z \rightarrow Y) \in \Sigma_{j}-\Sigma_{j-1}$ has been inferred by application of one of the four inference rules in $\mathfrak{F}$. If $\diamond(Z \rightarrow Y)$ results from the reflexivity axiom, then $Y \subseteq Z \subseteq$ Closure, where $Z \subseteq$ Closure follows from the induction hypothesis. If $\diamond(Z \rightarrow Y)$ results from applying the decomposition rule to $\diamond(Z \rightarrow Y U) \in \Sigma_{j-1}$, then $Y \subseteq Y U \subseteq$ Closure, where $Y U \subseteq$ Closure follows from the induction hypothesis. If $\diamond(Z \rightarrow Y)$ results from applying the union rule to $\diamond(Z \rightarrow U) \in \Sigma_{j-1}$ and $\diamond(Z \rightarrow V) \in \Sigma_{j-1}$, then $Y=U V \subseteq$ Closure, where $U V \subseteq$ Closure follows from the induction hypothesis. If $\diamond(Z \rightarrow Y)$ results from applying the null transitivity rule to $\diamond(Z \rightarrow U) \in$ $\Sigma_{j-1}$ and $\diamond(U \rightarrow Y) \in \Sigma_{j-1}$, then $U \subseteq Z R_{s}$. In particular, if $Z \subseteq$ Closure $\cap$ $X R_{s}$, then the hypothesis tells us that $U \subseteq$ Closure and $U \subseteq Z R_{s} \subseteq X R_{s}$. Applying the hypothesis to $\diamond(U \rightarrow Y) \in \Sigma_{j-1}$ and $U \subseteq$ Closure $\cap X R_{s}$ results in $Y \subseteq$ Closure. This concludes the proof. 
We now establish the worst-case linear time complexity of deciding the implication problem for WUCs and WFDs over Codd tables with a nullfree subschema. For that purpose, we first define the sizes of the measures we use. The size $|\varphi|$ of a WUC or WFD $\varphi$ is the total number of attributes occurring in $\varphi$, and the size $\|\Sigma\|$ of $\Sigma$ is the sum of $|\sigma|$ over all elements $\sigma \in \Sigma$. Further, let

$$
\Sigma[U]:=\{\diamond(V \rightarrow W) \in \Sigma \mid V \subseteq U\} \cup\{\diamond \mathcal{u}(V) \in \Sigma \mid V \subseteq U\}
$$

Theorem 3.4. The problem whether a WUC $\varphi=\diamond u(X)$ or WFD $\varphi=\diamond(X \rightarrow$ $Y)$ is implied by a set $\Sigma$ of WUCs and WFDs over relation schema $R$ with NFS $n f s\left(R_{s}\right)$ can be decided in $\mathcal{O}\left(\|\Sigma\|+\left\|\Sigma\left[X R_{s}\right] \cup\{\varphi\}\right\|\right)$ time.

Proof. Corollary 3.2 shows how to reduce the implication problem to the computation of the attribute closure with respect to $\Sigma[\mathrm{WFD}]$ and $n f_{s}\left(R_{s}\right)$. Algorithm 3.1 can be sped up by first computing the set $\Sigma\left[X R_{s}\right]$ by a single pass over $\Sigma$, given $\varphi=\diamond \mathfrak{u}(X)$ or $\varphi=\diamond(X \rightarrow Y)$, and $n f_{s}\left(R_{s}\right)$. The test for attributes to be in Closure $\cap X R_{s}$ of line 5 in Algorithm 3.1 then reduces to a test for attributes to be in Closure. Every attribute in $\Sigma\left[X R_{s}\right] \cup\{\varphi\}$ is then used at most once during the repeat loop.

We conclude this section by analyzing the examples from the previous section from an algorithmic point of view.

Example 3.5. Consider the relation schema WORK with attributes Emp, Dept and $M g r$, NFS Work WDept $_{s}=\{g r\}$, and

$$
\Sigma=\{\diamond(E m p \rightarrow D e p t), \diamond(D e p t \rightarrow M g r), \diamond u(M g r)\}
$$

Then $\Sigma \models_{\text {WORK }_{s}} \diamond \mathfrak{u}(E m p)$. Indeed, Algorithm 3.1 computes

$$
\{E m p\}_{\Sigma[W F D], \text { WoRK }_{s}}^{*}=\{E m p, \text { Dept }, M g r\}
$$

and $\diamond u(M g r) \in \Sigma$ where $\{M g r\} \subseteq\{E m p\} \cup$ WORK $_{s}$. Corollary 3.2 tells us therefore that $\Sigma \models$ WORK $_{s} \diamond \mathfrak{u}(E m p)$. 
Example 3.6. Consider the relation schema WORK with attributes Emp, Dept and $M g r$, NFS WORK $_{s}=\{$ Dept, $M g r\}$, and

$$
\Sigma=\{\diamond(E m p \rightarrow D e p t), \diamond(D e p t \rightarrow M g r)\}
$$

Then $\Sigma \not \models_{\text {WORK }_{s}} \diamond \mathcal{u}(E m p)$. Indeed, Algorithm 3.1 computes

$$
\{E m p\}_{\Sigma[W F D], \text { WORK }_{s}}^{*}=\{E m p, \text { Dept }, M g r\}
$$

but there is no WUC in $\Sigma$. Therefore, Corollary 3.2 tells us that $\Sigma \forall_{\text {WORK }_{s}}$ $\diamond u(E m p)$.

Example 3.7. Consider the relation schema WORK with attributes Emp, Dept and $M g r$, NFS WoRK $s=\{$ Dept $\}$, and

$$
\Sigma=\{\diamond(E m p \rightarrow D e p t), \diamond(D e p t \rightarrow M g r), \diamond u(M g r)\}
$$

Then $\Sigma \not \models_{\text {WORK }_{s}} \diamond \mathcal{u}(E m p)$. Indeed, Algorithm 3.1 computes

$$
\{E m p\}_{\Sigma[W F D], \text { WORK }_{s}}^{*}=\{E m p, \text { Dept }, M g r\}
$$

but for $\diamond u(M g r) \in \Sigma$ we have $\{M g r\} \nsubseteq\{E m p\} \cup$ WORK $_{s}$. Therefore, Corollary 3.2 tells us that $\Sigma \forall_{\text {WORK }_{s}} \diamond \mathcal{u}(\operatorname{Emp})$.

\subsection{Logical Characterization}

In this section we establish yet another characterization of the implication problem for the combined class of WUCs and WFDs over Codd tables with NFSs. The characterization is logical in the sense that these dependencies interact in the same way as Horn clauses do in Cadoli and Schaerf's well-known family of approximation logics $\mathcal{S}-3$. The set $\mathfrak{S}$ contains those propositional variables that are interpreted classically, that is, a variable is interpreted true if and only if the negated variable is interpreted false. However, variables outside of $\mathcal{S}$ can be true while their negation is true as well. Indeed, propositional variables in $\mathcal{S}$ correspond to the attributes that 
belong to the null-free subschema. If the null-free subschema contains all the attributes of the underlying relation schema, then we recover Fagin's equivalence between the implication problem of functional dependencies over relations and that of Horn clauses under classical Boolean implication. Our characterization refines this equivalence further: indeed, functional dependencies correspond to definite clauses and keys correspond to goal clauses.

\subsection{1 $\mathcal{S}$-3 Logics}

Schaerf and Cadoli [111] introduced $\mathcal{S}$-3 logics as "a semantically wellfounded logical framework for sound approximate reasoning, which is justifiable from the intuitive point of view, and to provide fast algorithms for dealing with it even when using expressive languages".

For a finite set $L$ of propositional variables let $L^{\ell}$ denote the set of all literals over $L$, i.e., $L^{\ell}=L \cup\left\{\neg A^{\prime} \mid A^{\prime} \in L\right\} \subseteq L^{*}$ where $L^{*}$ denotes the propositional language over $L$. Let $\mathcal{S} \subseteq L$. An $\mathcal{S}$-3 interpretation of $L$ is a total function $\omega: L^{\ell} \rightarrow\{\mathbb{F}, \mathbb{T}\}$ that maps every variable $A^{\prime} \in \mathcal{S}$ and its negation $\neg A^{\prime}$ into opposite values $\left(\omega\left(A^{\prime}\right)=\mathbb{T}\right.$ if and only if $\left.\omega\left(\neg A^{\prime}\right)=\mathbb{F}\right)$, and that does not map both a variable $A^{\prime} \in L-\mathcal{S}$ and its negation $\neg A^{\prime}$ into $\mathbb{F}$ (we must not have $\omega\left(A^{\prime}\right)=\mathbb{F}=\omega\left(\neg A^{\prime}\right.$ ) for any $A^{\prime} \in L-\mathcal{S}$ ). An $\mathcal{S}$-3 interpretation $\omega: L^{\ell} \rightarrow\{\mathbb{F}, \mathbb{T}\}$ of $L$ can be lifted to a total function $\Omega$ : $L^{*} \rightarrow\{\mathbb{F}, \mathbb{T}\}$ by means of simple rules [111]. Since we are only interested in Horn clauses here we require the following two rules for assigning truth values to a Horn clause:

1. $\Omega\left(\varphi^{\prime}\right)=\omega\left(\varphi^{\prime}\right)$, if $\varphi^{\prime} \in \mathcal{L}^{\ell}$, and

2. $\Omega\left(\varphi^{\prime} \vee \psi^{\prime}\right)=\mathbb{T}$ if and only if $\Omega\left(\varphi^{\prime}\right)=\mathbb{T}$ or $\Omega\left(\psi^{\prime}\right)=\mathbb{T}$.

An $\mathcal{S}-3$ interpretation $\omega$ is a model of a set $\Sigma^{\prime}$ of $L$-formulae if and only if $\Omega\left(\sigma^{\prime}\right)=\mathbb{T}$ holds for every $\sigma^{\prime} \in \Sigma^{\prime}$. We say that $\Sigma^{\prime} \mathcal{S}$-3 implies an $L$-formula $\varphi^{\prime}$, denoted by $\Sigma^{\prime} \models_{\mathcal{S}}^{3} \varphi^{\prime}$, if and only if every $\mathcal{S}$-3 interpretation that is a model of $\Sigma^{\prime}$ is also a model of $\varphi^{\prime}$. 
Example 3.8. Let $L=\left\{A^{\prime}, B^{\prime}, C^{\prime}\right\}, \mathcal{S}=\left\{A^{\prime}, C^{\prime}\right\}, \Sigma^{\prime}=\left\{\neg A^{\prime} \vee B^{\prime}, \neg B^{\prime} \vee C^{\prime}\right\}$, and $\varphi^{\prime}=\neg A^{\prime} \vee C^{\prime}$. The mappings that assign $\mathbb{T}$ to $B^{\prime}$ and $\neg B^{\prime}$, and $\mathbb{F}$ to $\neg A^{\prime}$ and $C^{\prime}$ define $\mathcal{S}-3$ interpretations that are models of $\Sigma^{\prime}$, but no models of $\varphi^{\prime}$. Therefore, $\Sigma^{\prime} \not \not_{\mathcal{S}}^{3} \varphi^{\prime}$.

However, if $\mathcal{S}=\left\{B^{\prime}\right\}$, then $\Sigma^{\prime} \models_{\mathcal{S}}^{3} \varphi^{\prime}$. Indeed, if an $\mathcal{S}$-3 interpretation violates $\varphi^{\prime}$, then it must map $\neg A^{\prime}$ and $C^{\prime}$ to $\mathbb{F}$. Since $B^{\prime} \in \mathcal{S}$, exactly one of $B^{\prime}$ and $\neg B^{\prime}$ must be mapped to $\mathbb{F}$, also violating $\Sigma^{\prime}$.

\subsubsection{Equivalence of Implication Problems}

In a first step, we define the fragment of $L$-formulae that corresponds to WUCs and WFDs in the presence of an NFS $n f_{s}\left(R_{s}\right)$ over a relation schema $R$. Let $\phi: R \rightarrow L$ denote a bijection between $R$ and the set $L=\left\{A^{\prime} \mid A \in R\right\}$ of propositional variables that corresponds to $R$. For an NFS $n f S\left(R_{s}\right)$ over $R$ let $\mathcal{S}=\phi\left(R_{s}\right)$ be the set of propositional variables in $L$ that corresponds to $R_{s}$. Hence, the variables in $\mathcal{S}$ are the images of those attributes of $R$ declared NOT NULL.

We now extend $\phi$ to a mapping $\Phi$ from the set of WUCs and WFDs over $R$. For a WUC $\diamond \mathfrak{u}\left(A_{1}, \ldots, A_{n}\right)$ over $R$, let $\Phi\left(\diamond u\left(A_{1}, \ldots, A_{n}\right)\right)$ denote the goal clause $\neg A_{1}^{\prime} \vee \cdots \vee \neg A_{n}^{\prime}$. For a WFD $\diamond\left(A_{1}, \ldots, A_{n} \rightarrow A\right)$ over $R$, let $\Phi\left(\diamond\left(A_{1}, \ldots, A_{n} \rightarrow A\right)\right)$ denote the definite clause $\neg A_{1}^{\prime} \vee \cdots \vee \neg A_{n}^{\prime} \vee A^{\prime}$. For the sake of presentation, but without loss of generality, we assume that WFDs have only a single attribute on their right-hand side. As usual, disjunctions over zero disjuncts are interpreted as $\mathbb{F}$. In what follows, we may simply denote $\Phi(\varphi)=\varphi^{\prime}$ and $\Phi(\Sigma)=\left\{\sigma^{\prime} \mid \sigma \in \Sigma\right\}=\Sigma^{\prime}$.

Our aim is to show that for every relation schema $R$, for every set $\Sigma \cup$ $\{\varphi\}$ of WUCs and WFDs and for every NFS $n f_{s}\left(R_{s}\right)$ over $R$, there is some $R_{s}$-total Codd table $r$ that satisfies $\Sigma$ and violates $\varphi$ if and only if there is an $\mathcal{S}$-3 model $\omega_{r}$ of $\Sigma^{\prime}$ that is not an $\mathcal{S}-3$ model of $\varphi^{\prime}$. For arbitrary Codd tables $r$ it is not obvious how to define the $\mathcal{S}-3$ interpretation $\omega_{r}$.

However, for deciding the implication problem $\Sigma \models_{R_{s}} \varphi$ it suffices to 
examine two-tuple Codd tables (instead of arbitrary Codd tables).

For two-tuple Codd tables $\left\{t_{1}, t_{2}\right\}$ we define the special-3-interpretation of $L$ by

$$
\begin{aligned}
& \omega_{\left\{t_{1}, t_{2}\right\}}\left(A^{\prime}\right)=\left\{\begin{array}{l}
\mathbb{F}, \text { if unk } \neq t_{1}(A) \neq t_{2}(A) \neq \text { unk } \\
\mathbb{T}, \text { otherwise }
\end{array}\right. \text { and } \\
& \omega_{\left\{t_{1}, t_{2}\right\}}\left(\neg A^{\prime}\right)=\left\{\begin{array}{l}
\mathbb{F}, \text { if unk } \neq t_{1}(A)=t_{2}(A) \neq \text { unk } \\
\mathbb{T}, \text { otherwise }
\end{array}\right.
\end{aligned}
$$

for all $A^{\prime} \in L$. In particular, if $\left\{t_{1}, t_{2}\right\}$ is $R_{s}$-total, then $\omega_{\left\{t_{1}, t_{2}\right\}}$ is an $\mathcal{S}-3$ interpretation, as the following proposition shows. The reverse direction is true, as well.

Proposition 3.2. For all relation schemata $R$, for all two-tuple Codd tables $r=$ $\left\{t_{1}, t_{2}\right\}$ over $R$, and for all NFSs $n f_{S}\left(R_{s}\right)$ over $R, r$ satisfies $n f_{s}\left(R_{s}\right)$ if and only if $\omega_{r}$ is an $\mathcal{S}$-3 interpretation of $L$.

Proof. If $r$ satisfies $n f_{S}\left(R_{s}\right)$, then $r$ is $R_{s}$-total. According to the definition of the special-3-interpretation $\omega_{r}$ it holds that for all variables $A^{\prime} \in \mathcal{S}$ we have $\omega_{r}\left(A^{\prime}\right)=\mathbb{T}$ if and only if $\omega_{r}\left(\neg A^{\prime}\right)=\mathbb{F}$, and for all variables in $L-\mathcal{S}$ we cannot have $\omega_{r}\left(A^{\prime}\right)=\mathbb{F}$ and $\omega_{r}\left(\neg A^{\prime}\right)=\mathbb{F}$.

If $\omega_{r}$ is an $\mathcal{S}-3$ interpretation of $L$, then for each variable $A^{\prime} \in \mathcal{S}$, either $\omega_{r}\left(A^{\prime}\right)=\mathbb{T}$ or $\omega_{r}\left(\neg A^{\prime}\right)=\mathbb{T}$ (but not both). According to the definition of $\omega_{r}$, however, $r$ must be $A$-total.

The definition of the special-3-interpretation is justified semantically by the following lemma.

Lemma 3.4. For all relation schemata $R$, for all two-tuple Codd tables $r=$ $\left\{t_{1}, t_{2}\right\}$ over $R$, and for all WUCs and all WFDs $\varphi$ over $R, r$ satisfies $\varphi$ and $n f s\left(R_{s}\right)$ if and only if $\omega_{r}$ is an $\mathcal{S}-3$ model of $\varphi^{\prime}$.

Proof. We show first that if $r$ satisfies $\varphi$ and $n f_{S}\left(R_{s}\right)$, then $\omega_{r}$ is an $\mathcal{S}$-3 model of $\varphi^{\prime}$. Since $r$ satisfies $n f s\left(R_{s}\right)$, Proposition 3.2 tells us that $\omega_{r}$ is an $\mathcal{S}-3$ interpretation. If $\varphi$ denotes the WUC $\diamond \mathcal{u}\left(A_{1}, \ldots, A_{n}\right)$, then $\varphi^{\prime}=\neg A_{1}^{\prime} \vee \cdots \vee$ 
$\neg A_{n}^{\prime}$. Since $r$ satisfies $\diamond u\left(A_{1}, \ldots, A_{n}\right)$ it cannot be that for all $i=1, \ldots, n$, unk $\neq t_{1}\left(A_{i}\right)=t_{2}\left(A_{i}\right) \neq$ unk hold. Therefore, it cannot be that for all $i=1, \ldots, n, \omega_{r}\left(\neg A_{i}^{\prime}\right)=\mathbb{F}$ holds. Hence, $\omega_{r}$ is an $\mathcal{S}$-3 model of $\varphi^{\prime}$. If $\varphi$ denotes the WFD $\diamond\left(A_{1} \cdots A_{n} \rightarrow A\right)$, then $\varphi^{\prime}=\neg A_{1}^{\prime} \vee \cdots \vee \neg A_{n}^{\prime} \vee A$. Since $r$ satisfies $\diamond\left(A_{1} \cdots A_{n} \rightarrow A\right)$ it cannot be that for all $i=1, \ldots, n$, unk $\neq$ $t_{1}\left(A_{i}\right)=t_{2}\left(A_{i}\right) \neq$ unk hold and unk $\neq t_{1}(A) \neq t_{2}(A) \neq$ unk holds, too. Therefore, it cannot be that for all $i=1, \ldots, n, \omega_{r}\left(\neg A_{i}^{\prime}\right)=\mathbb{F}$ hold and $\omega_{r}\left(\neg A^{\prime}\right)=\mathbb{F}$ holds, too. Hence, $\omega_{r}$ is an $\mathcal{S}$-3 model of $\varphi^{\prime}$.

We show next that if $\omega_{r}$ is an $\mathcal{S}$-3 model of $\varphi^{\prime}$, then $r$ satisfies $\varphi$ and $n f \mathcal{S}\left(R_{s}\right)$. Since $\omega_{r}$ is an $\mathcal{S}-3$ interpretation, Proposition 3.2 tells us that $r$ satisfies $n f s\left(R_{s}\right)$. If $\varphi^{\prime}$ denotes the goal clause $\neg A_{1}^{\prime} \vee \cdots \vee \neg A_{n}^{\prime}$, then $\varphi$ denotes the WUC $\diamond \mathcal{u}\left(A_{1}, \ldots, A_{n}\right)$. Since $\omega_{r}$ is an $\mathcal{S}$-3 model of $\neg A_{1}^{\prime} \vee \cdots \vee$ $\neg A_{n}^{\prime}$ it cannot be that for all $i=1, \ldots, n, \omega_{r}\left(\neg A_{i}^{\prime}\right)=\mathbb{F}$ holds. Consequently, it cannot be that for all $i=1, \ldots, n$, unk $\neq t_{1}\left(A_{i}\right)=t_{2}\left(A_{i}\right) \neq$ unk hold. Hence, $r$ satisfies the WUC $\diamond \mathcal{u}\left(A_{1}, \ldots, A_{n}\right)$. If $\varphi^{\prime}$ denotes the definite clause $\neg A_{1}^{\prime} \vee \cdots \vee \neg A_{n}^{\prime} \vee A^{\prime}$, then $\varphi$ denotes the WFD $\diamond\left(A_{1}, \ldots, A_{n} \rightarrow A\right)$. Since $\omega_{r}$ is an $\mathcal{S}$-3 model of $\neg A_{1}^{\prime} \vee \cdots \vee \neg A_{n}^{\prime} \vee A^{\prime}$ it cannot be that for all $i=$ $1, \ldots, n, \omega_{r}\left(\neg A_{i}^{\prime}\right)=\mathbb{F}$ hold and $\omega_{r}\left(A^{\prime}\right)=\mathbb{F}$ holds, too. Consequently, it cannot be that for all $i=1, \ldots, n$, unk $\neq t_{1}\left(A_{i}\right)=t_{2}\left(A_{i}\right) \neq$ unk hold and unk $\neq t_{1}(A) \neq t_{2}(A) \neq$ unk holds, too. Hence, $r$ satisfies the WFD $\diamond\left(A_{1}, \ldots, A_{n} \rightarrow A\right)$. This concludes the proof.

We are now in a position to establish the anticipated equivalence between the implication problem of WUCs and WFDs over Codd tables with an NFS and the implication problem of goal and definite clauses in Cadoli and Schaerf's $\mathcal{S}-3$ logic. The main results we rely on in the following proof are Corollary 3.1 and Lemma 3.4 .

Theorem 3.5. Let $\Sigma \cup\{\varphi\}$ be a set of WUCs and WFDs over the relation schema $R$, and let $n f S\left(R_{s}\right)$ denote an NFS over $R$. Let $L$ denote the set of propositional variables that corresponds to $R, \mathcal{S}$ the set of variables that corresponds to $R_{s}$, and $\Sigma^{\prime} \cup\left\{\varphi^{\prime}\right\}$ the set of goal and definite clauses over $L$ that corresponds to $\Sigma \cup\{\varphi\}$. Then $\Sigma \models_{R_{s}} \varphi$ if and only if $\Sigma^{\prime} \models_{\mathcal{S}}^{3} \varphi^{\prime}$. 
Proof. According to Corollary 3.1 it suffices to show that $\Sigma \models_{2, R_{s}} \varphi$ if and only if $\Sigma^{\prime}=_{\mathcal{S}}^{3} \varphi^{\prime}$.

We show first that if $\Sigma^{\prime} \models_{\mathcal{S}}^{3} \varphi^{\prime}$ holds, then $\Sigma \models_{2, R_{s}} \varphi$ holds as well. For this purpose suppose that $\Sigma \models_{2, R_{s}} \varphi$ does not hold. Consequently, there is some two-tuple Codd table $r$ over $R$ that satisfies $\Sigma$ and $n f s\left(R_{s}\right)$, but violates $\varphi$. Following Lemma 3.4. $\omega_{r}$ is an $\mathcal{S}-3$ interpretation that satisfies $\Sigma^{\prime}$ and violates $\varphi^{\prime}$. Hence, $\Sigma^{\prime} \models_{\mathcal{S}}^{3} \varphi^{\prime}$ does also not hold.

It now remains to show that if $\Sigma \models_{2, R_{s}} \varphi$ holds, then $\Sigma^{\prime} \models_{\mathcal{S}}^{3} \varphi^{\prime}$ holds as well. For this purpose, suppose that $\Sigma^{\prime} \models_{\mathcal{S}}^{3} \varphi^{\prime}$ does not hold. Consequently, there is some $\mathcal{S}-3$ interpretation $\omega$ that is a model of $\Sigma^{\prime}$ but not a model of $\varphi^{\prime}$. Let $r=\left\{t_{1}, t_{2}\right\}$ be defined as follows: for all $A \in R$ let $t_{1}(A) \in$ $\operatorname{dom}(A)-\{$ unk $\}$, and let $t_{2}(A)=t_{1}(A)$, if $\omega\left(A^{\prime}\right)=\mathbb{T}$ and $\omega\left(\neg A^{\prime}\right)=\mathbb{F}$, let $t_{2}(A)=$ unk, if $\omega\left(A^{\prime}\right)=\mathbb{T}=\omega\left(\neg A^{\prime}\right)$, and let $t_{2}(A) \in \operatorname{dom}(A)-\left\{t_{1}(A)\right.$, unk $\}$, if $\omega\left(A^{\prime}\right)=\mathbb{F}$ and $\omega\left(\neg A^{\prime}\right)=\mathbb{T}$. It follows that $\omega_{r}=\omega$. Since $\omega_{r}=\omega$, Lemma 3.4 guarantees that $r$ satisfies $\Sigma$ and $n f s\left(R_{s}\right)$, and $r$ violates $\varphi$. We conclude that $\Sigma \not \models_{R_{s}} \varphi$.

We conclude this section by analyzing the running examples from this chapter from a logical point of view.

Example 3.9. Consider the relation schema WORK with attributes Emp, Dept and $M g r$, NFS WORK $s=\{$ Dept, Mgr $\}$, and

$$
\Sigma=\{\diamond(E m p \rightarrow D e p t), \diamond(D e p t \rightarrow M g r), \diamond u(M g r)\}
$$

As we have demonstrated already, $\Sigma \models_{\text {WORK }_{s}} \diamond \mathcal{u}(E m p)$. In terms of $\mathcal{S}-3$ logics, the example translates as follows:

- $L=\left\{E m p^{\prime}\right.$, Dept $\left.^{\prime}, M g r^{\prime}\right\}$

- $\mathcal{S}=\left\{D e p t^{\prime}, M g r^{\prime}\right\}$

- $\Sigma^{\prime}=\left\{\neg E m p^{\prime} \vee D e p t^{\prime}, \neg D_{e p t^{\prime}} \vee M g r^{\prime}, \neg M g r^{\prime}\right\}$

- $\varphi^{\prime}=\neg E m p^{\prime}$. 
It is relatively straightforward to see that $\Sigma^{\prime} \models_{\mathcal{S}}^{3} \varphi^{\prime}$ holds, too. Suppose that for some $\mathcal{S}$-3 interpretation $\omega$ of $L, \omega\left(\neg E m p^{\prime}\right)=\mathbb{F}$ holds. If we want $\omega$ to be an $\mathcal{S}-3$ model of $\Sigma^{\prime}$ we must necessarily have $\omega\left(\right.$ Dept $\left.^{\prime}\right)=\mathbb{T}$. Since Dept $^{\prime} \in \mathcal{S}$, this means that $\omega\left(\neg D e p t^{\prime}\right)=\mathbb{F}$, which means that $\omega\left(M g r^{\prime}\right)=\mathbb{T}$. However, $M g r^{\prime} \in \mathcal{S}$, and to satisfy the goal clause $\neg M g r^{\prime}$ we must have $\omega\left(\neg M g r^{\prime}\right)=\mathbb{T}$. These requirements are contradictory. Consequently, there is no $\mathcal{S}$-3 interpretation of $L$ that is a model of $\Sigma^{\prime}$ and not a model of $\varphi^{\prime}$.

Example 3.10. Consider the relation schema WORK with attributes Emp, Dept and $M g r$, NFS WORK $s=\{$ Dept, Mgr $\}$, and

$$
\Sigma=\{\diamond(E m p \rightarrow \text { Dept }), \diamond(\text { Dept } \rightarrow M g r)\} .
$$

As we have already seen beforehand, $\Sigma \nvdash_{\text {WORK }_{s}} \diamond \mathcal{u}(E m p)$. In terms of $\mathcal{S}-3$ logics, the example translates as follows:

- $L=\left\{E m p^{\prime}\right.$, Dept $\left.^{\prime}, M g r^{\prime}\right\}$

- $\mathcal{S}=\left\{D e p t^{\prime}, M g r^{\prime}\right\}$

- $\Sigma^{\prime}=\left\{\neg E m p^{\prime} \vee D e p t^{\prime}, \neg D e p t^{\prime} \vee M g r^{\prime}\right\}$

- $\varphi^{\prime}=\neg E m p^{\prime}$.

Consider the $\mathcal{S}-3$ interpretation $\omega$ of $L$ that assigns $\mathbb{T}$ to all variables, and $\mathbb{F}$ to their negations. It is straightforward to observe that $\omega$ is a model of $\Sigma^{\prime}$, but not a model of $\varphi^{\prime}$. Hence, $\Sigma^{\prime} \not_{\mathcal{S}}^{3} \varphi^{\prime}$. Note that the $\mathcal{S}$-3 interpretation $\omega$ is the special-3-interpretation $\omega_{r}$ for the two-tuple Codd table $r$ from Example 3.3 that satisfies $\Sigma, n f_{S}\left(\mathrm{WORK}_{s}\right)$ and violates $\varphi$.

Example 3.11. Consider the relation schema WORK with attributes Emp, Dept and $M g r$, NFS WORK $_{s}=\{$ Dept $\}$, and

$$
\Sigma=\{\diamond(E m p \rightarrow \text { Dept }), \diamond(D e p t \rightarrow M g r), \diamond \mathcal{u}(M g r)\}
$$

As we have already seen beforehand, $\Sigma \not_{\text {WORK }_{s}} \diamond \mathcal{u}(E m p)$. In terms of $\mathcal{S}$-3 logics, the example translates as follows: 
- $L=\left\{E m p^{\prime}\right.$, Dept $\left.^{\prime}, M g r^{\prime}\right\}$

- $\mathcal{S}=\left\{\right.$ Dept $\left.^{\prime}\right\}$

- $\Sigma^{\prime}=\left\{\neg E m p^{\prime} \vee D e p t^{\prime}, \neg D e p t^{\prime} \vee M g r^{\prime}, \neg M g r^{\prime}\right\}$

- $\varphi^{\prime}=\neg E m p^{\prime}$.

Consider the $\mathcal{S}-3$ interpretation $\omega$ of $L$ that assigns $\mathbb{T}$ to all variables, $\mathbb{F}$ to $\neg E m p^{\prime}$ and $\neg D e p t^{\prime}$, and $\mathbb{T}$ to $\neg M g r^{\prime}$. Again, it is straightforward to observe that $\omega$ is a model of $\Sigma^{\prime}$, but not a model of $\varphi^{\prime}$. Hence, $\Sigma^{\prime} \forall_{\mathcal{S}}^{3} \varphi^{\prime}$. Note that the $\mathcal{S}$-3 interpretation $\omega$ is the special-3-interpretation $\omega_{r}$ for the two-tuple Codd table $r$ from Example 3.4 that satisfies $\Sigma, n f_{\mathcal{S}}\left(\mathrm{WORK}_{s}\right)$ and violates $\varphi$.

\subsection{Further Comments}

In this chapter, axiomatic, algorithmic, and logical characterizations of the implication problem for the combined class of weak uniqueness and NOT NULL constraints, and weak functional dependencies over Codd tables have been established. Using the techniques from this chapter, similar characterizations of the implication problem for the combined class of uniqueness and NOT NULL constraints, and functional dependencies over SQL tables can be established. It is stressed here again that SQL tables are based on occurrences of the ni null marker, while Codd tables are based on occurrences of the unk null marker using a possible world semantics. Due to these differences in semantics it is not at all obvious that similar characterizations hold.

While the contribution of this chapter focuses on Codd tables, recent related work on constraints over SQL tables should be mentioned here again. Indeed, axiomatic, algorithmic, and logical characterizations of the implication problem for the combined class of functional and multivalued dependencies over subsumption-free SQL relations with NOT NULL constraints were established in [70]. These were extended to the combined 
class of uniqueness and NOT NULL constraints, and functional and multivalued dependencies over SQL tables [55, 92]. Multivalued dependencies, however, are beyond the scope of this thesis.

\subsection{Summary}

In this chapter we have started to address the first objective of this thesis, as stated in Chapter 2. We investigated the implication problem for the combined class of weak uniqueness constraints, weak functional dependencies and NOT NULL constraints over Codd tables. We established axiomatic, efficient algorithmic, and logical characterizations of the implication problem. These results were announced in [56] and complement findings in the research literature, in particular recent findings on the implication problem for the combined class of uniqueness and NOT NULL constraints, and functional and multivalued dependencies over SQL tables [55, 70, 92].

In the following chapter we will make extensive use of the axiomatic and algorithmic characterizations in order to describe structural and computational properties of Armstrong tables for the combined class of weak uniqueness constraints, weak functional dependencies and NOT NULL constraints over Codd tables. In later chapters, we will describe an implementation of these algorithms and those from the recent literature in a graphical user interface. 


\section{Chapter 4}

\section{Foundations - Part II: Armstrong Tables}

In this chapter structural and computational properties of Armstrong tables are investigated for the combined class $\mathcal{C}$ of weak uniqueness constraints and weak functional dependencies over Codd tables with NOT NULL constraints. As it turns out, this class does indeed enjoy Armstrong tables, in contrast to the combined class of uniqueness constraints and functional dependencies over SQL tables with NOT NULL constraints [64]. Indeed, the latter class had to be restricted to standard uniqueness constraints and standard functional dependencies to enjoy Armstrong tables. In Section 4.2 a structural characterization of Armstrong tables is established. That is, conditions are given that are sufficient and necessary for a given Codd table to be Armstrong with respect to a given set of constraints in $\mathcal{C}$. These conditions will be stipulated in terms of agree sets, maximal sets, and duplicate sets, similar to the notions used in the classical case of relations and the case of SQL tables. The structural characterization is then exploited in Section 4.3 to establish an algorithm that, given an arbitrary relation schema, an arbitrary null-free subschema and an arbitrary set of weak uniqueness constraints and weak functional dependencies over the schema, computes a Codd table that is Armstrong with respect to this in- 
put. Several properties are established that provide insight into the complexity of this algorithm. First of all, the time complexity of finding an Armstrong table is precisely exponential in the size of the input, as it already is in the idealized special case of Armstrong relations for functional dependencies. However, the algorithm does produce an Armstrong table whose size is at most quadratic in the number of rows in a minimum-sized Armstrong table. While there are cases where the size of a minimum-sized Armstrong table is exponential in the size of the constraints, there are also cases where the size of an Armstrong table is logarithmic in the size of the optimal cover of the input. The results of this chapter were announced in [56].

\subsection{Two Motivating Examples}

The chapter begins with a re-examination of the example preceding Theorem 2.9. which showed that there are sets of functional dependencies over SQL tables for which no Armstrong table exists. In the context of Codd tables, however, the same constraint set does admit Armstrong tables. In fact, it will turn out in this chapter that the entire class of WUCs, WFDs, and NFSs does enjoy Armstrong tables.

Example 4.1. Let WORK denote our running example of a relation schema with attributes Emp, Dept, and $M g r$, and let $\mathrm{WORK}_{s}=\{$ Dept, $M g r\}$ define the null-free subschema. Consider the set $\Sigma$ consisting of the WFDs $\diamond(\emptyset \rightarrow$ $E m p)$ and $\diamond(E m p \rightarrow D e p t)$. The Codd table

\begin{tabular}{ccc}
\hline Emp & Dept & Mgr \\
\hline Dilbert & IT & Gates \\
unk & Security & Gates \\
Dilbert & IT & Murdoc \\
Dilbert & IT & Gates \\
\hline
\end{tabular}


is an Armstrong table for $\Sigma$ and $n f_{s}\left(\right.$ WORK $\left._{s}\right)$ with respect to the combined class of WUCs and WFDs. Note, in particular, that $\diamond(\emptyset \rightarrow E m p)$ is satisfied, since the null marker occurrence unk can simply be replaced by Dilbert. Similarly, $\diamond(E m p \rightarrow$ Dept $)$ is satisfied, since the null marker occurrence unk can simply be replaced by a domain value different from Dilbert.

As a second motivating example we re-examine another one of our running examples from the point of view of WUCs and WFDs.

Example 4.2. Let WORK $=\{E m p$, Dept, $M g r\}$ and let $\mathrm{WORK}_{s}=\{E m p, M g r\}$ define the null-free subschema. Consider the set $\Sigma$ consisting of the WFDs $\diamond(E m p \rightarrow$ Dept $)$ and $\diamond($ Dept $\rightarrow M g r)$. The Codd table

\begin{tabular}{ccc}
\hline Emp & Dept & Mgr \\
\hline Dilbert & IT & Gates \\
Alice & IT & Gates \\
Dogbert & Security & Gates \\
Dilbert & unk & Murdoc \\
Dilbert & IT & Gates \\
\hline
\end{tabular}

is an Armstrong table for $\Sigma$ and $n f_{s}\left(\right.$ WORK $\left._{s}\right)$ with respect to the combined class of WUCs and WFDs. In contrast to the SQL table from Table 2.14 which is Armstrong for the analogous set of constraints of SQL tables, the Codd table above is smaller in size. As it will turn out in this chapter, the general construction proposed for Codd tables that are Armstrong will produce tables of approximately half the size of Armstrong tables for the analogous set of constraints over SQL tables.

\subsection{Structural Properties}

First we would like to establish sufficient and necessary conditions when a given Codd table is an Armstrong table with respect to a given set $\Sigma$ of WUCs and WFDs and an NFS $n f_{s}\left(R_{s}\right)$. This would generalize Theorem 2.4. a well-known result by Mannila, Räihä, Beeri, Dowd, Fagin and 
Statman for functional dependencies over total database relations [11, 96]. Especially useful in this regard is Mannila and Räihä's notion of maximal sets [96] which we generalize here from total relations to Codd tables.

Definition 4.1. Let $\Sigma$ be a set of WUCs and WFDs and let $n f f_{s}\left(R_{s}\right)$ be an NFS over relation schema $R$. For an attribute $A \in R$ we define the maximal sets $\max _{\Sigma, R_{s}}(A)$ of $A$ with respect to $\Sigma$ and $n f s\left(R_{s}\right)$ as follows:

$$
\begin{aligned}
& \max _{\Sigma, R_{s}}(A):=\left\{X \subseteq R \mid \Sigma \not \ell_{R_{s}} \diamond(X \rightarrow A) \wedge\right. \\
&\left.\forall B \in R-X\left(\Sigma \models_{R_{s}} \diamond(X B \rightarrow A)\right)\right\} .
\end{aligned}
$$

The maximal sets of $R$ with respect to $\Sigma$ and $n f_{S}\left(R_{s}\right)$ are defined as $\max _{\Sigma, R_{s}}(R)=$ $\bigcup_{A \in R} \max _{\Sigma, R_{s}}(A)$. If $\Sigma$ and $n f_{s}\left(R_{s}\right)$ are clear from the context we may simply write $\max (A)$ and $\max (R)$, respectively.

Thus, the maximal sets of an attribute $A$ with respect to $\Sigma$ and $n f_{s}\left(R_{s}\right)$ are the maximal attribute subsets of $R$ that do not functionally determine $A$. Note that Definition 4.1 permits the empty set to occur as a maximal set.

Example 4.3. Let WORK $=\{E m p$, Dept, Mgr $\}$ and let $\mathrm{WORK}_{s}=\{E m p, M g r\}$ define the null-free subschema. Consider the set $\Sigma$ consisting of the WFDs $\diamond(E m p \rightarrow D e p t)$ and $\diamond(D e p t \rightarrow M g r)$. Then the maximal sets for the attributes are:

- $\max _{\Sigma, R_{s}}(E m p)=\{\{$ Dept, Mgr $\}\}$,

- $\max _{\Sigma, R_{s}}($ Dept $)=\{\{M g r\}\}$,

- $\max _{\Sigma, R_{s}}(M g r)=\{\{E m p\}\}$.

Example 4.4. Consider the relation schema CONTACT with attributes Address, City and ZIP, and an NFS defined by $R_{s}=\mathrm{CONTACT}_{s}=\{Z I P\}$, and

$$
\Sigma=\{\diamond u(\text { Address, City }), \diamond(Z I P \rightarrow \text { City })\} .
$$

Then the maximal sets for the attributes are: 
- $\max _{\Sigma, R_{s}}($ Address $)=\{\{$ City, ZIP $\}\}$,

- $\max _{\Sigma, R_{s}}($ City $)=\{\{$ Address $\}$,

- $\max _{\Sigma, R_{s}}(Z I P)=\{\{$ Address $\},\{$ City $\}$.

For a Codd table to be Armstrong it is necessary that for each maximal set there must be distinct tuples in the table whose strong agree set is the maximal set. This is to guarantee that all the WFDs not implied by the set of WUCs and WFDs in the presence of an NFS are violated. Over tables, however, it is still possible that there are WUCs $\diamond \mathcal{u}(X)$ not implied by $\Sigma$ in the presence of $n f_{s}\left(R_{s}\right)$ over $R$, even if the WFD $\diamond(X \rightarrow R)$ is implied. For this reason we also require of Armstrong tables that for all attribute sets $X$ that are maximal with this property there must be distinct rows in the table whose strong agree set is $X$. This motivates the following definition.

Definition 4.2. Let $\Sigma$ be a set of WUCs and WFDs and let $n f s\left(R_{s}\right)$ be an NFS over relation schema $R$. We define the duplicate sets $\operatorname{dup}_{\Sigma, R_{s}}(R)$ of $R$ with respect to $\Sigma$ and $n f s\left(R_{s}\right)$ as follows:

$$
\begin{gathered}
\operatorname{dup}_{\Sigma, R_{s}}(R):=\left\{X \subseteq R\left|\Sigma \models_{R_{s}} \diamond(X \rightarrow R) \wedge \Sigma\right| f_{R_{s}} \diamond \mathcal{u}(X) \wedge\right. \\
\left.\forall B \in T-X\left(\Sigma \models_{R_{s}} \diamond \mathfrak{u}(X B)\right)\right\} .
\end{gathered}
$$

If $\Sigma$ and $n f s\left(R_{s}\right)$ are clear from the context we may simply write dup $(R)$.

Example 4.5. Let WORK $=\{E m p$, Dept, $M g r\}$ and let $\mathrm{WORK}_{s}=\{E m p, M g r\}$ define the null-free subschema. Consider the set $\Sigma$ consisting of the WFDs $\diamond(E m p \rightarrow$ Dept $)$ and $\diamond(D e p t \rightarrow M g r)$. Then we have $d u p_{\Sigma, R_{s}}($ WORK $)=$ $\{\{$ Emp, Dept, Mgr $\}$.

Example 4.6. Consider the relation schema CONTACT with attributes $A d-$ dress, City and ZIP, NFS defined by $R_{s}=$ CONTACT $_{s}=\{Z I P\}$, and

$$
\Sigma=\{\diamond u(\text { Address, City }), \diamond(Z I P \rightarrow \text { City })\}
$$

Then we have $d u p_{\Sigma, R_{s}}($ CONTACT $)=\{\{$ Address, ZIP $\}$. 
For our anticipated characterization of Armstrong tables the notion of a (strong) agree set plays an important role. While strong and weak agree sets coincide over total relations, the distinction between the two is crucial for Codd tables. Indeed, we require an additional notion that helps us to ensure that i) for each maximal set of an attribute there are tuples that strongly agree on the maximal set but disagree on the attribute, and ii) each strong agree set includes all attributes functionally determined by it.

Definition 4.3. Let $R$ be a relation schema, and $r$ a Codd table over $R$. For $X \in a g^{s}(r)$ let $w(X)=\bigcap\left\{Y \mid \exists t, t^{\prime} \in r\left(X=a g^{s}\left(t, t^{\prime}\right) \wedge Y=a g\left(t, t^{\prime}\right)\right)\right\}$.

Example 4.7. Let $r$ denote the following Codd table

\begin{tabular}{ccc}
\hline Address & City & ZIP \\
\hline Pont Neuf & Paris & 75001 \\
Le Louvre & Paris & 75001 \\
Pont Neuf & Toulouse & 31000 \\
unk & Paris & 75007 \\
Pont Neuf & unk & 75001 \\
\hline
\end{tabular}

Here we obtain

- $a g^{s}(r)=\{\{$ City, ZIP $\},\{$ Address $\},\{$ City $\},\{$ Address, ZIP $\}\}$,

- $a g^{w}(r)=\{\{$ Address $\},\{$ City $\}\}$, and

- $w($ Address $)=\{$ Address $\}, w($ City $)=\{$ City $\}, w($ City, ZIP $)=\{$ City, ZIP $\}$, and $w($ Address, ZIP $)=\{$ Address, City, ZIP $\}$.

These notions allow us to obtain the following characterization of Armstrong tables for a given Codd table. Conditions 1 . and 3. ensure that the Codd table violates all WFDs and WUCs not implied by $\Sigma$ and $n f_{s}\left(R_{s}\right)$, while conditions 2 . and 4 . ensure that the Codd table satisfies all WFDs and WUCs in $\Sigma$. Finally, condition 5. ensures that the Codd table is total on every attribute of the null-free subschema, and not total on any attribute not in the null-free subschema. 
Theorem 4.1. Let $R$ be a relation schema, $\Sigma$ a set of WUCs and WFDs, and $n f s\left(R_{s}\right)$ an NFS over $R$. For all Codd tables $r$ over $R$ it holds that $r$ is an Armstrong table for $\Sigma$ and $n f s\left(R_{s}\right)$ if and only if all of the following conditions are satisfied:

1. $\forall A \in R \forall X \in \max _{\Sigma, R_{s}}(A)\left(X \in a g^{s}(r) \wedge A \notin w(X)\right)$,

2. $\forall X \in a g^{s}(r)\left(X_{\Sigma, R_{s}}^{*} \subseteq w(X)\right)$,

3. $\forall X \in \operatorname{dup}_{\Sigma, R_{s}}(R)\left(X \in a g^{s}(r)\right)$,

4. $\forall X \in a g^{s}(r) \forall \diamond u(Z) \in \Sigma(Z \nsubseteq X)$,

5. $\operatorname{total}(r)=\{A \in R \mid \forall t \in r(t(A) \neq$ unk $)\}=R_{s}$.

Proof. Sufficiency. Let $r$ be some Codd table over $R$ that satisfies conditions 1., 2., 3., 4. and 5. We show that $r$ is an Armstrong table for $\Sigma$ and $n f s\left(R_{s}\right)$.

Let $\diamond(X \rightarrow A) \in \Sigma$. Assume that there are distinct $t, t^{\prime} \in r$ such that $t(X)=t^{\prime}(X)$ and $t$ is $X$-total. That is, $X \subseteq X^{\prime}=a g^{s}\left(t, t^{\prime}\right)$. Note that $A \in\left(X^{\prime}\right)_{\Sigma, R_{s}}^{*}$. Hence, condition 2. implies that $A \in w\left(X^{\prime}\right)$. In particular, $A \in \operatorname{ag}\left(t, t^{\prime}\right)$. According to Proposition 3.1, $r$ satisfies every WFD in $\Sigma$.

Let $\diamond u(X) \in \Sigma$, and let $t, t^{\prime} \in r$ be distinct tuples of $r$. Condition 4 . ensures that $X \nsubseteq a g^{s}\left(t, t^{\prime}\right)$ holds. Proposition 3.1 tells us that $r$ satisfies $\diamond \mathcal{u}(X)$. We conclude that $r$ satisfies $\Sigma$.

Let $\diamond(X \rightarrow A) \notin \Sigma_{R_{s}}^{*}$. It follows that there is some $X^{\prime} \in \max _{\Sigma, R_{s}}(A)$ such that $X \subseteq X^{\prime}$ and $A \notin\left(X^{\prime}\right)_{\Sigma, R_{s}}^{*}$. Condition 1. implies that $X^{\prime} \in a g^{s}(r)$ and $A \notin w\left(X^{\prime}\right)$. Hence, there is some $Y \in a g(r)$ such that $A \notin Y$. This shows that there are two distinct $t, t^{\prime} \in r$ such that $X \subseteq a g^{s}\left(t, t^{\prime}\right)$ and $A \notin a g\left(t, t^{\prime}\right)$. Proposition 3.1 tells us that $r$ violates the WFD $\diamond(X \rightarrow A)$.

Let $\diamond u(X) \notin \Sigma_{R_{s}}^{*}$. If $\diamond(X \rightarrow R) \notin \Sigma_{R_{s}}^{*}$, then we are in the previous case, which shows, in particular, that there are two distinct $t, t^{\prime} \in r$ such that $X \subseteq a g^{s}\left(t, t^{\prime}\right)$. Proposition 3.1 tells us that $r$ violates the WUC $\diamond u(X)$. Suppose now that $\diamond(X \rightarrow R) \in \Sigma_{R_{s}}^{*}$. Then there is some $X^{\prime} \in d u p_{\Sigma, R_{s}}(R)$ 
such that $X \subseteq X^{\prime}$. Condition 3. ensures that there are some $t, t^{\prime} \in r$ such that $X \subseteq X^{\prime} \subseteq a g^{s}\left(t, t^{\prime}\right)$. Again, Proposition 3.1 tells us that $r$ violates the WUC $\diamond \mathcal{u}(X)$. We have shown that $r$ violates every WUC and WFD that is not in $\Sigma_{R_{s}}^{*}$.

Finally, Condition 5. ensures that $r$ satisfies every NFS implied by $n f_{s}\left(R_{s}\right)$, and violates every NFS not implied by $n f_{s}\left(R_{s}\right)$. Consequently, $r$ is an Armstrong table for $\Sigma$ and $n f_{s}\left(R_{s}\right)$.

Necessity. Let $r$ be some Codd table over $R$ that is Armstrong for $\Sigma$ and $n f_{S}\left(R_{s}\right)$. We show that $r$ satisfies conditions 1., 2., 3., 4., and 5 .

Let $A \in R$, and let $X \in \max _{\Sigma, R_{s}}(A)$. That is, $\Sigma \nvdash_{R_{s}} \diamond(X \rightarrow A)$ and for all $B \in R-X$ it is true that $\Sigma \models_{R_{s}} \diamond(X B \rightarrow A)$. Since $r$ is an Armstrong table for $\Sigma$ and $n f_{S}\left(R_{s}\right)$ it follows that $r$ violates $\diamond(X \rightarrow A)$ and for all $B \in R-X$ that $r$ satisfies the WFD $\diamond(X B \rightarrow A)$. The violation of $\diamond(X \rightarrow A)$ implies that there are distinct $t, t^{\prime} \in r$ such that $X \subseteq a g^{s}\left(t, t^{\prime}\right)$ and $A \notin$ $a g\left(t, t^{\prime}\right)$, according to Proposition 3.1. If there was some attribute $C$ of $R$ in $a g^{s}\left(t, t^{\prime}\right)-X$, then $r$ would violate the WFD $\diamond(X C \rightarrow A)$. Consequently, $X=a g^{s}\left(t, t^{\prime}\right)$. We have just shown that for every $A \in R$ and for every $X \in \max _{\Sigma, R_{s}}(A)$ it is true that $X \in a g^{s}(r)$ and $A \notin w(X)$, i.e., condition 1 . holds.

Next we show that $r$ satisfies condition 2. Therefore, let $X \in a g^{s}(r)$. We need to show that $X_{\Sigma, R_{s}}^{*} \subseteq w(X)$. Let $A$ be some attribute of $R$ such that $A \notin w(X)$. Consequently, there are some distinct $t, t^{\prime} \in r$ such that $X=a g^{s}\left(t, t^{\prime}\right)$ and $A \notin a g\left(t, t^{\prime}\right)$. Again, Proposition 3.1 tells us that $r$ violates the WFD $\diamond(X \rightarrow A)$. Since $r$ is an Armstrong table for $\Sigma$ and $n f s\left(R_{s}\right)$ it follows that $A \notin X_{\Sigma, R_{s}}^{*}$. We have just shown that $X_{\Sigma, R_{s}}^{*} \subseteq w(X)$.

We show that $r$ satisfies condition 3. Therefore, let $X \in d u p_{\Sigma, R_{s}}(R)$. By definition, $\diamond \mathfrak{u}(X) \notin \Sigma_{R_{s}}^{*}$. As $r$ is an Armstrong table for $\Sigma$ and $n f_{s}\left(R_{s}\right)$, it follows that $r$ violates $\diamond \mathcal{u}(X)$. According to Proposition 3.1 there must be distinct $t, t^{\prime} \in r$ with $X \subseteq a g^{s}\left(t, t^{\prime}\right)$. Moreover, by definition of $X \in$ $\operatorname{dup}_{\Sigma, R_{s}}(R)$, it follows that for all $B \in R-X, \diamond \mathcal{u}(X B) \in \Sigma_{R_{s}}^{*}$. As $r$ is an Armstrong table for $\Sigma$ and $n f_{s}\left(R_{s}\right)$, it follows that $r$ satisfies $\diamond \mathcal{u}(X B)$ for 
all $B \in R-X$. Consequently, $X B \nsubseteq a g^{s}\left(t, t^{\prime}\right)$ for all $B \in R-X$. Hence, $X \in a g^{s}(r)$.

It is straightforward to see that $r$ satisfies condition 4 . Indeed, as $r$ is an Armstrong table for $\Sigma$ and $n f s\left(R_{s}\right), r$ satisfies every $\diamond \mathcal{u}(Z) \in \Sigma$. According to Proposition 3.1 there cannot be any $X \in a g^{s}(r)$ such that $Z \subseteq X$.

Since $r$ is an Armstrong table for $\Sigma$ and $n f s\left(R_{s}\right)$ it follows that total $(r)=$ $R_{s}$.

Note that Condition 1. of Theorem 4.1, i.e.,

$$
\forall A \in R \forall X \in \max _{\Sigma, R_{s}}(A)\left(X \in a g^{s}(r) \wedge A \notin w(X)\right)
$$

can be replaced by

$$
\forall A \in R \forall X \in \max _{\Sigma[\mathrm{WFD}], R_{s}}(A)\left(X \in a g^{s}(r) \wedge A \notin w(X)\right),
$$

following Lemma 3.2 .

Example 4.8. Consider again the relation schema CONTACT with headers Address, City and ZIP, NFS $n f s\left(R_{s}\right)$ defined by $R_{s}=\mathrm{CONTACT}_{s}=\{Z I P\}$, and

$$
\Sigma=\{\diamond u(\text { Address, City }), \diamond(Z I P \rightarrow \text { City })\}
$$

Examples 4.4, 4.6 and 4.7 allow us to verify all the conditions of Theorem 4.1 for the following Codd table $t$ :

\begin{tabular}{ccc}
\hline Address & City & ZIP \\
\hline Pont Neuf & Paris & 75001 \\
Le Louvre & Paris & 75001 \\
Pont Neuf & Toulouse & 31000 \\
unk & Paris & 75007 \\
Pont Neuf & unk & 75001 \\
\hline
\end{tabular}

Hence, Theorem 4.1 shows that $r$ is indeed an Armstrong table for $\Sigma$ and $n f s\left(R_{s}\right)$. 


\subsection{Computational Properties}

In this section we establish an algorithm that computes for any given relation schema $R$, any given set $\Sigma$ of WUCs and WFDs, and any given NFS $n f s\left(R_{s}\right)$ over $R$ a Codd table that is Armstrong for $\Sigma$ and $n f_{s}\left(R_{s}\right)$. The algorithm is based on the structural characterization of Armstrong tables in Theorem 4.1. For that purpose, we will compute the families of maximal sets, and the duplicate sets first, before we exploit these sets to construct tuples with appropriate agree sets that match the conditions in Theorem 4.1 .

\subsubsection{Computation of Maximal Sets}

Following Theorem 4.1 we aim to compute the maximal set families $\max _{\Sigma[\mathrm{WFD}], R_{s}}(A)$

for all $A \in R$, as well as the duplicate sets $\operatorname{dup}_{\Sigma, R_{s}}(R)$.

Lemma 4.1. Let $R$ be a relation schema, $n f_{S}\left(R_{s}\right)$ an NFS over $R$, and $\Sigma$ a set of $W U C s$ and WFDs over $R$. For $W C \subseteq R$, it takes $\mathcal{O}(|R| \times \| \Sigma||)$ time to test whether $W \in \max _{\Sigma[W F D], R_{S}}(C)$.

Proof. Using Algorithm 3.1. $C \notin W_{\Sigma[W F D], R_{s}}^{*}$ can be checked in time $\mathcal{O}(\|\Sigma\|)$, and $C \in(W B)_{\Sigma[\mathrm{WFD}], R_{s}}^{*}$ for all $B \in R-W$ can be checked in time $\mathcal{O}(|R| \times$ $\|\Sigma\|)$.

We use $\operatorname{mtest}\left(W, C, R, R_{s}, \Sigma\right)$ to denote the test if $W \in \max _{\Sigma[\mathrm{WFD}], R_{s}}(C)$ from Lemma 4.1. The maximal sets for $R$ with respect to $\Sigma[$ WFD] and $n f s\left(R_{s}\right)$ can be computed by testing all subsets of $R$. This, however, will hardly be efficient. The following result establishes an iterative approach for computing the maximal sets for $R$ with respect to $\Sigma[\mathrm{WFD}]$ and $n f_{s}\left(R_{s}\right)$. The algorithm starts with the maximal sets for $R$ with respect to an empty WFD set in the presence of $n f s\left(R_{s}\right)$, and then adds the WFDs of $\Sigma$ [WFD] 
one by one while monitoring the resulting changes to the family of maximal sets.

Theorem 4.2. Let $R$ be a relation schema, $n f s\left(R_{s}\right)$ an NFS over $R$, and $\Sigma^{\prime}=$ $\Sigma \cup\{\diamond(X \rightarrow A)\}$ a set of WFDs over $R$. For $C \in R$ let $V \in \max _{\Sigma, R_{s}}(C)$. Then $V \in \max _{\Sigma^{\prime}, R_{s}}(C)$ or $\left(C=A\right.$ or $\left.A \in R_{s}\right)$ holds and there is some $B \in X-V$ such that

i) $V B \in \max _{\Sigma^{\prime}, R_{s}}(C)$, if $X \nsubseteq R_{s}$, or

ii) $V=W \cap Z$ for some $W \in \max _{\Sigma^{\prime}, R_{s}}(C)$ and some $Z \in \max _{\Sigma^{\prime}, R_{s}}(B)$.

The proof of Theorem 4.2 uses the following simple observation:

Remark 4.1. Let $\Sigma=\Sigma^{\prime} \cup\{\diamond(X \rightarrow A)\}$ and $U \subseteq R$. When $A \in U_{\Sigma, R_{s}}^{*}$ we have

$$
U_{\Sigma, R_{s}}^{*}= \begin{cases}(U A)_{\Sigma, R_{s}}^{*}=(U A)_{\Sigma^{\prime}, R_{s}}^{*} & , \text { if } A \in R_{s} \\ U_{\Sigma^{\prime}, R_{s}}^{*} A & , \text { otherwise }\end{cases}
$$

while $U_{\Sigma, R_{s}}^{*}=U_{\Sigma^{\prime}, R_{s}}^{*}$ holds when $A \notin U_{\Sigma, R_{s}}^{*}$. Furthermore, the following statements are equivalent: a) $U_{\Sigma^{\prime}, R_{s}}^{*} \subset U_{\Sigma, R_{s}}^{*}$ b) $A \in U_{\Sigma, R_{s}}^{*}-U_{\Sigma^{\prime}, R_{s^{\prime}}}^{*}$ and c) $A \notin U_{\Sigma^{\prime}, R_{s}}^{*}$ and $X \subseteq U R_{s} \cap U_{\Sigma^{\prime}, R_{s}}^{*}, c f$. Algorithm 3.1

Proof. From $V \in \max _{\Sigma, R_{s}}(C)$ and $V \subseteq V_{\Sigma^{\prime}, R_{s}}^{*} \subseteq V_{\Sigma, R_{s}}^{*}$ we get $C \notin V_{\Sigma, R_{s}}^{*}$ and $C \notin V_{\Sigma^{\prime}, R_{s}}^{*}$. If $V \in \max _{\Sigma^{\prime}, R_{s}}(C)$ we are done. Otherwise, there is some $W \in$ $\max _{\Sigma^{\prime}, R_{s}}(C)$ with $V \subset W$. By $V \in \max _{\Sigma, R_{s}}(C)$ we obtain $C \in W_{\Sigma, R_{s}}^{*}-W_{\Sigma^{\prime}, R_{s^{\prime}}}^{*}$ so that $W_{\Sigma^{\prime}, R_{s}}^{*} \subset W_{\Sigma, R_{s}}^{*}$. When applying Remark 4.1 to the set $W$, we note $A \in W_{\Sigma, R_{s}}^{*}-W_{\Sigma^{\prime}, R_{s}}^{*}$ and, therefore, $\left(C=A\right.$ or $\left.A \in R_{s}\right)$.

Remark 4.1 for $W$ further yields $A \notin W_{\Sigma^{\prime}, R_{s}}^{*}$ and $X \subseteq W R_{s} \cap W_{\Sigma^{\prime}, R_{s}}^{*}$. Hence, $A \notin V_{\Sigma^{\prime}, R_{s}}^{*}$ as $V \subset W$. Assume $A \in V_{\Sigma, R_{s}}^{*}-V_{\Sigma^{\prime}, R_{s}}^{*}$. Then, $C \neq A$ as $C \notin V_{\Sigma, R_{s}}^{*}$, and thus $A \in R_{s}$. When applying Remark 4.1 to the set $V$, we observe $V_{\Sigma, R_{s}}^{*}=(V A)_{\Sigma, R_{s}}^{*}=(V A)_{\Sigma^{\prime}, R_{s}}^{*}$. Hence, $C \notin(V A)_{\Sigma, R_{s}}^{*}$. By $V \in \max _{\Sigma, R_{s}}(C)$ we obtain $A \in V$. Thus $A \in V \subseteq W \subseteq W_{\Sigma^{\prime}, R_{s}}^{*}$ which contradicts $A \in W_{\Sigma, R_{s}}^{*}-W_{\Sigma^{\prime}, R_{s}}^{*}$. Hence, our assumption is false, and $A \notin$ $V_{\Sigma, R_{s}}^{*}$ holds. 
From $A \notin V_{\Sigma, R_{s}}^{*}$ and Remark 4.1 for $V$ we conclude $V_{\Sigma, R_{s}}^{*}=V_{\Sigma^{\prime}, R_{s}}^{*}$ and $X \nsubseteq V R_{s} \cap V_{\Sigma^{\prime}, R_{s}}^{*}$, that is, $\left(X \nsubseteq V R_{s}\right.$ or $\left.X \nsubseteq V_{\Sigma^{\prime}, R_{s}}^{*}\right)$. By $X \subseteq W R_{s}$ we obtain $\left(\left(X-R_{s}\right) \cap(W-V) \neq \emptyset\right.$ or $\left.X \nsubseteq V_{\Sigma^{\prime}, R_{s}}^{*}\right)$. Therefore, $V$ is a subset of a member $U$ of

$$
\begin{aligned}
\mathcal{V}:= & \left\{W-B \mid B \in X-R_{s}\right\} \cup \\
& \left\{W \cap Z \mid W \nsubseteq Z \in \cup_{B \in X} \max _{\Sigma^{\prime}, R_{s}}(B)\right\} .
\end{aligned}
$$

From $U \subset W$ and $W \in \max _{\Sigma^{\prime}, R_{s}}(C)$ we get $C \notin U_{\Sigma^{\prime}, R_{s}}^{*}$. By definition of $\mathcal{V}$, we observe $X \nsubseteq U R_{s} \cap U_{\Sigma^{\prime}, R_{s}}^{*}$ which yields $U_{\Sigma, R_{s}}^{*}=U_{\Sigma^{\prime}, R_{s}}^{*}$ by Remark 4.1. Thus, we obtain $C \notin U_{\Sigma, R_{s}}^{*}$. From $V \in \max _{\Sigma, R_{s}}(C)$ we derive $V=U$, that is, $V$ itself is a member of $\mathcal{V}$. This concludes the proof of Theorem 4.2

Theorem 4.2 shows that Algorithm 4.1 computes the family of maximal sets.

Theorem 4.3. On input $\left(R, \Sigma, R_{s}\right)$, Algorithm 4.1 computes the sets $\max _{\Sigma, R_{s}}(C)$ for all $C \in R$.

We illustrate the iterative approach towards computing the maximal set families on our running examples.

Example 4.9. Let WORK $=\{E m p$, Dept, Mgr $\}$ and let $R_{s}=\mathrm{WORK}_{s}=$ $\{E m p, M g r\}$ define the null-free subschema. Consider the set $\Sigma$ consisting of the WFDs $\diamond(E m p \rightarrow$ Dept $)$ and $\diamond(D e p t \rightarrow M g r)$. The computation

\begin{tabular}{cccc}
\hline$A$ & $\max _{\emptyset, R_{s}}(A)$ & $\max _{\{\diamond(E m p \rightarrow \text { Dept })\}, R_{s}}(A)$ & $\max _{\Sigma, R_{s}}(A)$ \\
\hline Emp & $\{\{$ Dept,$M g r\}\}$ & $\{\{$ Dept,$M g r\}\}$ & $\{\{$ Dept,$M g r\}\}$ \\
Dept & $\{\{E m p, M g r\}\}$ & $\{\{M g r\}\}$ & $\{\{M g r\}\}$ \\
$M g r$ & $\{\{E m p$, Dept $\}\}$ & $\{\{$ Emp,Dept $\}\}$ & $\{\{E m p\}\}$ \\
\hline
\end{tabular}

shows how Algorithm 4.1 may compute the maximal set families.

Example 4.10. Consider the relation schema CONTACT with attributes Address, City and ZIP, and an NFS defined by $R_{s}=$ CONTACT $_{s}=\{Z I P\}$, and

$$
\Sigma=\{\diamond u(\text { Address, City }), \diamond(Z I P \rightarrow \text { City })\}
$$




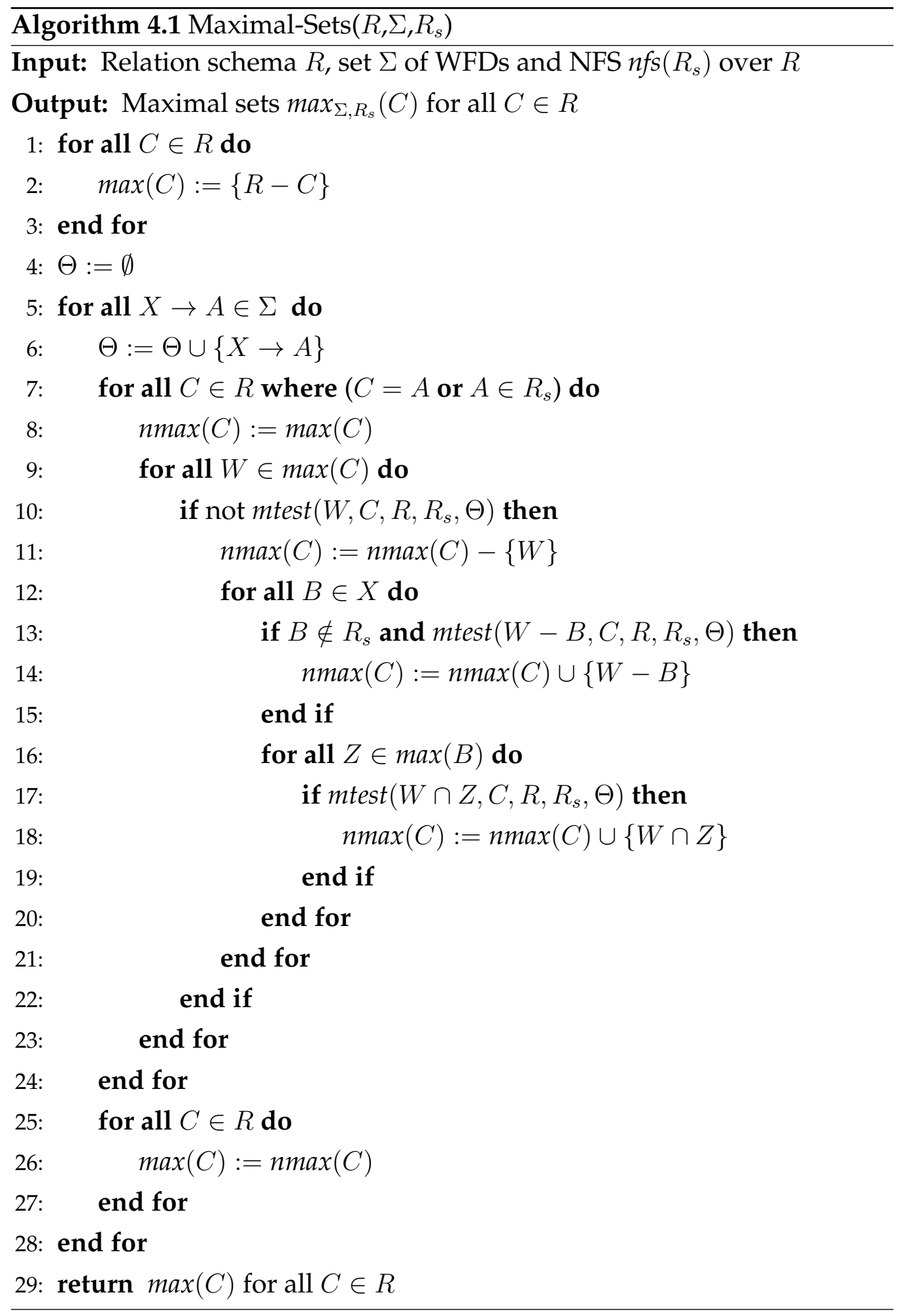


Let $\sigma$ denote the WFD $\diamond($ Address, City $\rightarrow$ ZIP $)$. The computation

\begin{tabular}{cccc}
\hline$A$ & $\max _{\emptyset, R_{s}}(A)$ & $\max _{\{\sigma\}, R_{s}}(A)$ & $\max _{\Sigma, R_{s}}(A)$ \\
\hline Address & $\{\{$ City, ZIP $\}$ & $\{\{$ City,$Z I P\}\}$ & $\{\{$ City,$Z I P\}\}$ \\
City & $\{\{$ Address,$Z I P\}\}$ & $\{\{$ Address,$Z I P\}\}$ & $\{\{$ Address $\}\}$ \\
ZIP & $\{\{$ Address, City $\}$ & $\{\{$ Address $\},\{$ City $\}$ & $\{\{$ Address $\},\{$ City $\}$ \\
\hline
\end{tabular}

shows how Algorithm 4.1 may compute the maximal set families.

\subsubsection{Computation of Duplicate Sets}

The next goal is to compute the set of duplicate sets, given a relation schema $R$, an NFS $n f s\left(R_{s}\right)$ and a set $\Sigma$ of WUCs and WFDs over $R$. For this purpose we exploit hyper-graphs and minimal transversals of hypergraphs.

Recall that a hypergraph $\mathcal{H}=(V, E)$ consists of a set $V$ of vertices and a set $E$ of subsets of $V$. The elements of $E$ are called hyper-edges. A transversal of $\mathcal{H}$ is a subset $\mathcal{T} \subseteq V$ such that $\mathcal{T} \cap H \neq \emptyset$ holds for every $H \in$ $E$. A transversal $\mathcal{T}$ of $\mathcal{H}$ is said to be minimal if and only if no proper subset of $\mathcal{T}$ is also a transversal of $\mathcal{H}$. Let $\operatorname{Tr}(\mathcal{H})$ denote the set of all minimal transversals of the hyper-graph $\mathcal{H}$. Research on the computation of $\operatorname{Tr}(\mathcal{H})$ is advanced [59].

We will now identify the relationship between duplicate sets and minimal transversals of a suitable hyper-graph obtained from the given set of WUCs and WFDs, and the given NFS.

Lemma 4.2. Let $R$ be some relation schema, $n f s\left(R_{s}\right)$ an NFS over $R$, and $\Sigma$ a set of WUCs and WFDs over $R$. For every $X \subseteq R$ where $\Sigma \models_{R_{s}} \diamond(X \rightarrow R)$ the following holds: $\Sigma \models_{R_{s}} \diamond \mathcal{u}(X)$ if and only if there is some $\diamond \mathcal{u}(K) \in \Sigma$ such that $K-R_{s} \subseteq X$.

Proof. If there is some $\diamond \mathfrak{u}(K) \in \Sigma$ such that $K-R_{s} \subseteq X$, then $\Sigma \models_{R_{s}} \diamond \mathfrak{u}(X)$ is a consequence due to the soundness of the null pullback rule. 
Vice versa, if for all $\diamond u(K) \in \Sigma, K \nsubseteq X R_{s}$, then the Codd table

\begin{tabular}{cc}
\hline$X R_{s}$ & $R-X R_{s}$ \\
\hline $0 \cdots 0$ & $0 \cdots 0$ \\
$0 \cdots 0$ & unk $\cdots$ unk
\end{tabular}

shows that $\Sigma \not \nvdash_{R_{s}} \diamond \mathcal{U}(X)$.

Given relation schema $R$, the NFS $n f s\left(R_{s}\right)$, and the set $\Sigma$ of WUCs and WFDs over $R$, we generate the hyper-graph $\mathcal{H}=(V, E)$ with vertex set $V=R$ and the set

$$
E=\left\{K-R_{s} \mid \diamond u(K) \in \Sigma\right\}
$$

of hyper-edges. The following result shows how the set of duplicate sets can be computed from the set of minimal transversals of the associated hyper-graph.

Theorem 4.4. Let $R$ be some relation schema, $n f_{s}\left(R_{s}\right)$ an NFS over $R$, and $\Sigma$ a set of WUCs and WFDs over $R$. Then $\operatorname{dup}_{\Sigma, R_{\mathrm{s}}}(R)=\{R-X \mid X \in \operatorname{Tr}(\mathcal{H}) \wedge \forall M \in$ $\left.\max _{\Sigma[W F D], R_{s}}(R)(R-X \nsubseteq M)\right\}$.

Proof. We show that $X \in \operatorname{dup}_{\Sigma, R_{s}}(R)$ if and only if $R-X \in \operatorname{Tr}(\mathcal{H})$ and $X \nsubseteq M$ for all $M \in \max _{\Sigma[\mathrm{WFD}], R_{s}}(R)$.

By definition, $X \in \operatorname{dup}_{\Sigma, R_{s}}(R)$ if and only if $\Sigma \models_{R_{s}} \diamond(X \rightarrow R)$ and $\Sigma \not R_{R_{s}} \diamond \mathcal{u}(X)$ and for all $A \in R-X, \Sigma \mid=_{R_{s}} \diamond \mathcal{u}(X A)$.

It is straightforward to observe that $\Sigma \models_{R_{s}} \diamond(X \rightarrow R)$ if and only if for all $M \in \max _{\Sigma[\mathrm{WFD}], R_{s}}(R), X \nsubseteq M$ holds.

Therefore, $X \in d u p_{\Sigma, R_{s}}(R)$ if and only if for all $M \in \max _{\Sigma, R_{s}}(R), X \nsubseteq$ $M$, and $\Sigma \not \models_{R_{s}} \diamond \mathcal{u}(X)$ and for all $A \in R-X, \Sigma \models_{R_{s}} \diamond \mathcal{u}(X A)$.

However, Lemma 4.2 shows that $\Sigma \forall_{R_{s}} \diamond \mathcal{u}(X)$ if and only if for all $\diamond \mathcal{u}(K) \in \Sigma,\left(K-R_{s}\right) \cap(R-X) \neq \emptyset$ holds.

Furthermore, Lemma 4.2 shows that for all $A \in R-X, \Sigma=_{R_{s}} \diamond u(X A)$ if and only if for all $A \in R-X,\left(K-R_{s}\right) \cap((R-X)-A)=\emptyset$ holds. 
Therefore, $\Sigma \not \models_{R_{s}} \diamond \mathfrak{u}(X)$ and for all $A \in R-X, \Sigma \models_{R_{s}} \diamond \mathfrak{u}(X A)$ hold if and only if $R-X$ is a minimal transversal of the hyper-graph $\mathcal{H}=(V, E)$ with vertex set $T=R$ and hyper-edge set $E=\left\{K-R_{s} \mid \diamond \mathcal{u}(K) \in \Sigma\right\}$. This concludes the proof.

Theorem 4.4 shows that the following Algorithm computes the set of all duplicate sets. The computation of the minimal transversals in line 3 can be done with any algorithm known from the research literature to this well-studied problem [59]. The computation of the maximal set families can be accomplished by Algorithm 4.1 .

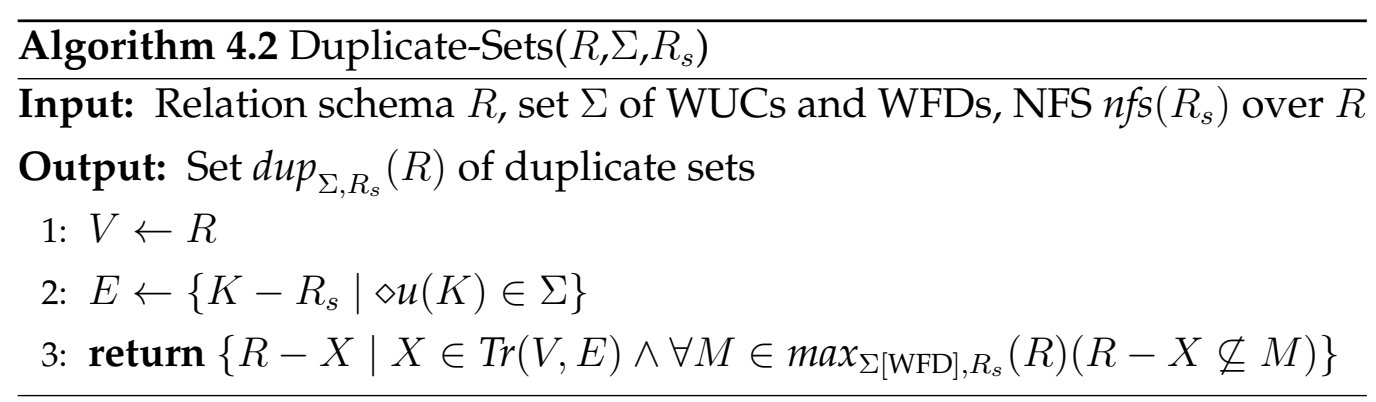

The correctness of Algorithm 4.2 follows immediately from Theorem 4.4.

Corollary 4.1. On input $\left(R, \Sigma, R_{s}\right)$, Algorithm 4.2 computes the set dup ${\overline{\Sigma, R_{s}}}_{(}(R)$ of duplicate sets.

We illustrate the computation of duplicate sets on one of our running examples.

Example 4.11. Consider again the relation schema CONTACT with headers Address, City and ZIP, NFS $n f_{s}\left(R_{s}\right)$ defined by $R_{s}=\mathrm{CONTACT}_{s}=\{Z I P\}$, and

$$
\Sigma=\{\diamond u(\text { Address, City }), \diamond(Z I P \rightarrow \text { City })\}
$$

Let $\left(\right.$ CONTACT, $\Sigma$, CONTACT $_{s}$ ) be input to Algorithm 4.2. Then we compute: 
- $V=\{$ Address, City, ZIP $\}$

- $E=\{\{$ Address, City $\}$

- $\operatorname{Tr}(V, E)=\{\{$ Address $\},\{$ City $\}\}$

- $\operatorname{dup}_{\Sigma, R_{s}}($ CONTACT $)=\{\{$ Address,ZIP $\}\}$ since $\{$ City, ZIP $\}$ is already (contained in) a maximal set of CONTACT, see Example 4.4 .

This was the same result we had already announced in Example 4.6 .

\subsubsection{Computation of Armstrong Tables}

The following algorithm computes an Armstrong table for an arbitrary set $\Sigma$ of WUCs and WFDs on an arbitrary relation schema $R$ with arbitrary NFS $n f s\left(R_{s}\right)$. If the non-standard WUC $\diamond u(\emptyset)$ is part of the input, Algorithm 4.3 returns in line 3 a Codd table with a single row that is unk on attributes in $R-R_{s}$ and total elsewhere. Otherwise, Algorithm 4.3 starts with a base tuple $t_{0}$ that is total on every attribute (line 7), computes the set of maximal sets for each attribute by Algorithm 4.1 in line 8, computes the duplicate sets by Algorithm 4.2 in line 10, and determines the set Const of constant columns in line 11 by Algorithm 3.1. The latter are determined by the attribute closure of the empty set of attributes. The main construction of the Armstrong table is occurring between lines 13 and 22. Indeed, Algorithm 4.3 adds a new row for each maximal set and duplicate set. For each maximal set $X$, the set $Z$ of attributes is determined in line 14 for which that set is maximal. Lines 19 and 20 then add a row whose strong agree set with a base tuple is just the current maximal set, which disagrees with the base tuple on all the attributes in $Z$ and in $R_{s}-(X \cup$ Const $)$, and which weakly agrees with the base tuple on all remaining attributes. For each duplicate set $X$, this set $Z$ is empty and $R_{s}$ is contained in $X$. That is, for each duplicate set, lines 19 and 20 add a row that strongly agrees with the base tuple on $X$ and weakly agrees with the base tuple everywhere else. Finally, lines 23-29 first determine whether the current Codd 
table features occurrences of unk in all attributes in $R-R_{s}$. If that is not the case, then a new tuple is inserted that ensures such occurrences before the Codd table is returned. Otherwise, the Codd table is returned straightaway. The output of Algorithm 4.3 is always an Armstrong table that matches the conditions of Theorem 4.1 . The correctness of Algorithm 4.3 follows essentially from Theorems $4.1,4.2$ and 4.4 .

Theorem 4.5. On input $\left(R, \Sigma, R_{s}\right)$, Algorithm 4.3 computes a Codd table that is Armstrong for $\Sigma$ and $n f_{s}\left(R_{s}\right)$.

Proof. For the proof, we verify that the output $r$ of Algorithm 4.3 satisfies the conditions in Theorem 4.1.

If $\diamond u(\emptyset) \in \Sigma$, then $r$ returned by line 3 is an Armstrong table, as there are no agree sets, maximal sets or duplicate sets, and for all $A \in R, r$ features null marker occurrences in $A$ if and only if $A \in R_{s}$. For the remainder of the proof we assume that $\diamond \mathfrak{u}(\emptyset) \notin \Sigma$. For condition 1 . let $X \in \max _{\Sigma[\mathrm{WFD}], R_{s}}(A)$. Due to lines 13-18, we have $A \in Z$ and lines 19-20 insert a new tuple in $r$ that agrees strongly with the tuple $t_{0}$ on $X$. Moreover, as $A \in Z$, lines 19-20 further guarantee that $A \notin w(X)$.

For condition 2. let $X \in a g^{s}(r)$, and let $\Sigma \models_{R_{s}} \diamond(X \rightarrow A)$ for some $A \in R-X$. We need to show that $A \in w(X)$. If $X \in a g^{s}(r)$, then i) $X \in \max _{\Sigma[\mathrm{WFD}], R_{s}}(R)$, ii) $X \in \operatorname{dup}_{\Sigma, R_{s}}(R)$, or iii) $X=$ Const $\cap R_{s}$. In case i) we have $A \notin Z$, as otherwise $X \in \max _{\Sigma[\mathrm{WFD}], R_{s}}(A)$ would contradict $\Sigma \models_{R_{s}} \diamond(X \rightarrow A)$. Also in case i), if $A \in R_{s}$, then $\Sigma \models_{R_{s}} \diamond(X \rightarrow A)$ and $X \in \max _{\Sigma[\mathrm{WFD}], R_{s}}(B)$ contradict one another. Indeed, the latter implies $\Sigma \models_{R_{s}} \diamond(X A \rightarrow B)$, but together with the former and $A \in R_{s}$ we also get $\Sigma \models_{R_{s}} \diamond(X \rightarrow B)$, contradicting $X \in \max _{\Sigma[\mathrm{WFD}], R_{s}}(B)$. Hence, $A \notin R_{s}$. Thus, case i) implies that $A \in w(X)$ by the construction in lines 19-20. Case ii) immediately implies $A \in w(X)$ by the construction in line 21 as then $Z=\emptyset$ and $X \subseteq R_{s}$, holds. Case iii) may occur by the construction in lines 25-26. As $X_{\Sigma, R_{s}}^{*}=$ Const we also have $A \in w(X)$ due to the construction in lines 25-26. 


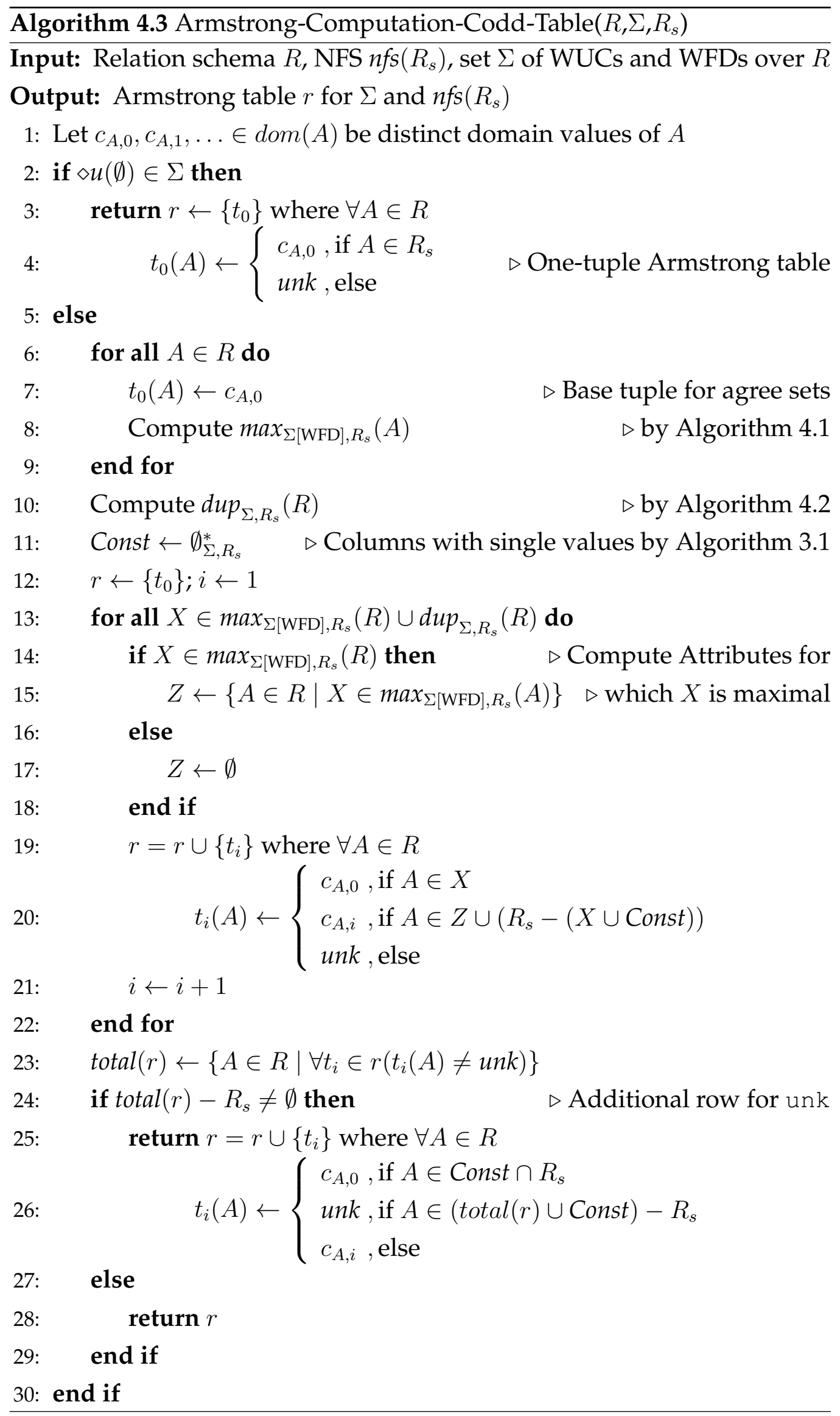


For condition 3. let $X \in \operatorname{dup}_{\Sigma, R_{s}}(R)$. It follows that $X \notin \max _{\Sigma[\mathrm{WFD}], R_{s}}(R)$ and therefore $Z=\emptyset$. Furthermore, if there was some $A \in R_{s}-X$, then $\Sigma \models R_{R_{s}} \diamond \mathcal{u}(X A)$ and $\Sigma \models_{R_{s}} \diamond(X \rightarrow A)$, and the soundness of the pullback rule would imply that $\Sigma \models_{R_{s}} \diamond \mathcal{u}(X)$ held, too. This would be a contradiction to $X \in \operatorname{dup}_{\Sigma, R_{s}}(R)$, and therefore, $R_{s} \subseteq X$. Hence, lines 19-20 insert a new tuple in $r$ that agrees strongly with the tuple $t_{0}$ on $X$.

For condition 4. let $X \in a g^{s}(r)$ and $\diamond \mathcal{u}(Z) \in \Sigma$. By construction, $X \in$ $a g^{s}(r)$ means that i) $X \in \max _{\Sigma[\mathrm{WFD}], R_{s}}(R)$, ii) $X \in \operatorname{dup}_{\Sigma, R_{s}}(R)$, or iii) $X=$ Const $\cap R_{s}$. Since $\diamond \mathcal{u}(Z) \in \Sigma$, we cannot have case i) and we cannot have case ii). Case iii) would imply that $\diamond \mathfrak{u}(\emptyset) \in \Sigma$, which cannot occur in this branch of the if command from line 2 .

Condition 5. is ensured by lines 23-29.

We conclude this section by revisiting our running examples.

Example 4.12. Let WORK denote our running example of a relation schema with attributes Emp, Dept, and $M g r$, and let $R_{s}=\mathrm{WORK}_{s}=\{D e p t, M g r\}$ define the null-free subschema. Consider the set $\Sigma$ consisting of the WFDs $\diamond(\emptyset \rightarrow E m p)$ and $\diamond(E m p \rightarrow D e p t)$. The maximal sets of WORK with respect to $\Sigma$ and $n f_{s}\left(R_{s}\right)$ are:

- $\max _{\Sigma, R_{s}}(E m p)=\emptyset$

- $\max _{\Sigma, R_{s}}($ Dept $)=\{\{M g r\}\}$

- $\max _{\Sigma, R_{s}}(M g r)=\{\{E m p, D e p t\}\}$.

Furthermore, $\{E m p$, Dept, $M g r\}$ is the only duplicate set of WORK with respect to $\Sigma$ and $n f s\left(R_{s}\right)$. Algorithm 4.3 would compute the Armstrong table on the left for $\Sigma$ and $n f_{S}\left(R_{s}\right)$.

\begin{tabular}{ccc}
\hline Emp & Dept & $M g r$ \\
\hline$c_{E, 0}$ & $c_{D, 0}$ & $c_{M, 0}$ \\
unk & $c_{D, 1}$ & $c_{M, 0}$ \\
$c_{E, 0}$ & $c_{D, 0}$ & $c_{M, 1}$ \\
$c_{E, 0}$ & $c_{D, 0}$ & $c_{M, 0}$ \\
\hline
\end{tabular}

\begin{tabular}{ccc}
\hline Emp & Dept & Mgr \\
\hline Dilbert & IT & Gates \\
unk & Security & Gates \\
Dilbert & IT & Murdoc \\
Dilbert & IT & Gates \\
\hline
\end{tabular}


A suitable substitution results in the Armstrong table on the right, already shown in Example 4.1 .

Example 4.13. Consider our relation schema WORK $=\{E m p, D e p t, M g r\}$ and let $\mathrm{WORK}_{s}=\{E m p, M g r\}$ define the null-free subschema. Consider the set $\Sigma$ consisting of the WFDs $\diamond(E m p \rightarrow$ Dept $)$ and $\diamond(D e p t \rightarrow M g r)$. Examples 4.3 and 4.5 show the families of maximal and duplicate sets for $\Sigma$ and $n f s\left(R_{s}\right)$. Algorithm 4.3 would compute the Armstrong table on the left for $\Sigma$ and $n f_{s}\left(R_{s}\right)$.

\begin{tabular}{ccc}
\hline Emp & Dept & Mgr \\
\hline$c_{E, 0}$ & $c_{D, 0}$ & $c_{M, 0}$ \\
$c_{E, 1}$ & $c_{D, 0}$ & $c_{M, 0}$ \\
$c_{E, 2}$ & $c_{D, 2}$ & $c_{M, 0}$ \\
$c_{E, 0}$ & unk & $c_{M, 3}$ \\
$c_{E, 0}$ & $c_{D, 0}$ & $c_{M, 0}$ \\
\hline
\end{tabular}

\begin{tabular}{ccc}
\hline Emp & Dept & Mgr \\
\hline Dilbert & IT & Gates \\
Alice & IT & Gates \\
Dogbert & Security & Gates \\
Dilbert & unk & Murdoc \\
Dilbert & IT & Gates \\
\hline
\end{tabular}

A suitable substitution results in the Armstrong table on the right, already shown in Example 4.2 .

Example 4.14. Consider the relation schema CONTACT with attributes $A d-$ dress, City and ZIP, NFS $n f s\left(R_{s}\right)$ defined by $R_{s}=\mathrm{CONTACT}_{s}=\{Z I P\}$, and

$$
\Sigma=\{\diamond u(\text { Address, City }), \diamond(Z I P \rightarrow \text { City })\} .
$$

Examples 4.4 and 4.6 show the families of maximal and duplicate sets for $\Sigma$ and $n f_{s}\left(R_{s}\right)$. Algorithm 4.3 would compute the Armstrong table on the left for $\Sigma$ and $n f_{S}\left(R_{s}\right)$.

\begin{tabular}{ccc}
\hline Address & City & ZIP \\
\hline$c_{A, 0}$ & $c_{C, 0}$ & $c_{Z, 0}$ \\
$c_{A, 1}$ & $c_{C, 0}$ & $c_{Z, 0}$ \\
$c_{A, 0}$ & $c_{C, 2}$ & $c_{Z, 2}$ \\
unk & $c_{C, 0}$ & $c_{Z, 3}$ \\
$c_{A, 0}$ & unk & $c_{Z, 0}$ \\
\hline
\end{tabular}

\begin{tabular}{ccc}
\hline Address & City & ZIP \\
\hline Pont Neuf & Paris & 75001 \\
Le Louvre & Paris & 75001 \\
Pont Neuf & Toulouse & 31000 \\
unk & Paris & 75007 \\
Pont Neuf & unk & 75001 \\
\hline
\end{tabular}


A suitable substitution results in the Armstrong table on the right, already shown in Example 4.8.

\subsection{Complexity Considerations}

The main objective of this section is to show that the increase in expressivity by going from Armstrong relations to Armstrong tables comes at no increased costs in terms of the efficiency of creating them. For this purpose, it is shown first that the problem of finding an Armstrong table for a given set of WUCs and WFDs and a given NFS remains precisely exponential in the size of the input, just as it was the case for relations [11]. It is further shown that our Algorithm 4.3 for computing Armstrong tables is rather conservative in the sense that the size of the computed Armstrong table is at most quadratic in the minimum size of the output possible, similar to the special case of relations [96]. Subsequently, we examine the most concise way of representing the information inherent in a set of WUCs, WFDs and NFSs. Indeed, already in the case of relations neither the representation in form of an abstract constraint set nor the representation in form of an Armstrong table strictly dominates the other. It is therefore advisable to exploit both representations in practice. In fact, it will turn out in the results of subsequent chapters that abstract constraints are best used to identify constraints that are incorrectly perceived as semantically meaningless, while Armstrong tables are best used to identify constraints that are incorrectly perceived as semantically meaningful.

\subsubsection{Worst-case Complexity to Find Armstrong Tables}

The user-friendly representation of an abstract constraint set in form of an Armstrong table comes, in the worst case, at a high price. In fact, the number of tuples in a minimum-sized Armstrong table can be exponential in the number of attributes. Due to this result we cannot expect to design 
an algorithm for generating Armstrong tables in polynomial time in the worst case. The next result shows that the number of attribute sets maximal for $\Sigma$ and $n f s\left(R_{s}\right)$ added by the number of duplicate sets is a lower bound for the number of agree sets found in any Armstrong table for $\Sigma$ and $n f s\left(R_{s}\right)$. A similar result holds for the special case of relations [11].

Proposition 4.1. Let $\Sigma$ be a set of WUCs and WFDs and $n f s\left(R_{s}\right)$ be some NFS over relation schema $R$, and let $r$ be an Armstrong table for $\Sigma$ and $n f_{s}\left(R_{s}\right)$. Then

$$
\left|\max _{\Sigma, R_{s}}(R) \cup \operatorname{dup}_{\Sigma, R_{s}}(R)\right| \leq|\operatorname{ag}(r)| \leq\left(\begin{array}{c}
|r| \\
2
\end{array}\right) .
$$

Proof. The first and third conditions of Theorem 4.1 implies that

$$
\left|\max _{\Sigma, R_{s}}(R) \cup \operatorname{dup}_{\Sigma, R_{s}}(R)\right| \leq\left|a g^{s}(r)\right|
$$

Moreover, $|\operatorname{ag} s(r)| \leq|\operatorname{ag}(r)|$, and $|\operatorname{ag}(r)| \leq\left(\begin{array}{c}|r| \\ 2\end{array}\right)$ since every distinct pair of distinct tuples in $r$ has precisely one agree set.

We recall what we mean by precisely exponential [11]. Firstly, it means that there is an algorithm for computing an Armstrong table, given a set $\Sigma$ of WUCs and WFDs over relation schema $R$ with an NFS $n f S\left(R_{s}\right)$, where the running time of the algorithm is exponential in the size of $\Sigma$. Secondly, it means that there is a set $\Sigma$ of WUCs and WFDs and an NFS $n f_{s}\left(R_{s}\right)$ in which the number of rows in each minimum-sized Armstrong table for $\Sigma$ and $n f s\left(R_{s}\right)$ is exponential, so it requires an exponential amount of time simply to write down the table.

Proposition 4.2. The complexity of finding an Armstrong table for a given set $\Sigma$ of WUCs and WFDs over a relation schema $R$ with null-free subschema $n f_{s}\left(R_{s}\right)$ is precisely exponential in the size of $\Sigma$.

Proof. The time complexity of Algorithm 4.3 is dominated by that of Algorithms 4.1 and 4.2 which run clearly in time exponential in the number of attributes [11, 59, 96]. 
It remains to show that there is a set $\Sigma$ of WUCs and WFDs and an NFS $n f_{s}\left(R_{s}\right)$ for which the number of tuples in each Armstrong table for $\Sigma$ and $n f_{S}\left(R_{s}\right)$ is exponential in the number of attributes. According to Proposition 4.1 it suffices to find a set $\Sigma$ of WUCs and WFDs such that $\max _{\Sigma, R_{s}}(R)$ is exponential in the number of attributes. Such a set $\Sigma$ is given by

$$
\bigcup_{1 \leq i \leq n}\left\{\diamond\left(\left\{A_{2 i-1}, A_{2 i}\right\} \rightarrow B\right)\right\}
$$

and the NFS defined by $R_{s}=A_{1} \cdots A_{2 n} B$. This is the same set that Beeri, Dowd, Fagin and Statman used to show that the time complexity of finding an Armstrong relation for FDs over relations takes at least exponential time in the number of attributes [11]. This set works here for the same purpose since all WFDs in $\Sigma$ have the same right-hand side.

\subsubsection{Minimum-sized Armstrong Tables}

The previous section has shown that the general worst-case time complexity of Algorithm 4.3 is exponential in the number of attributes. It is demonstrated now that, despite this worst-case exponential time complexity, Algorithm 4.3 is a fairly simple algorithm for generating Armstrong tables that is conservative in its use of time.

Let the size of an Armstrong table be defined as the number of tuples that it contains. In practice, the most appealing Armstrong table for an abstract constraint set $\Sigma$ should be of minimum size. The reason is that a small number of tuples is easier to comprehend for humans. Therefore, it is a practical question to ask how many tuples a minimum-sized Armstrong table requires. An Armstrong table $r$ for $\Sigma$ and $n f s\left(R_{s}\right)$ is said to be minimum-sized if there is no Armstrong table $r^{\prime}$ for $\Sigma$ and $n f_{s}\left(R_{s}\right)$ such that $\left|r^{\prime}\right|<|r|$. That is, for a minimum-sized Armstrong table for $\Sigma$ and $n f_{s}\left(R_{s}\right)$ there is no Armstrong table for $\Sigma$ and $n f_{s}\left(R_{s}\right)$ with a smaller number of tuples. 
Proposition 4.3. Let $\Sigma$ be a set of WUCs and WFDs, and $n f s\left(R_{s}\right)$ be some NFS over relation schema $R$. Let $r$ be a minimum-sized Armstrong table for $\Sigma$ and $n f s\left(R_{s}\right)$. Then

$$
\frac{\sqrt{1+8 \cdot\left|\max _{\Sigma, R_{s}}(R) \cup d u p_{\Sigma, R_{s}}(R)\right|}}{2} \leqslant|r| \leqslant\left|\max _{\Sigma, R_{s}}(R) \cup d u p_{\Sigma, R_{s}}(R)\right|+2 .
$$

Proof. The lower bound follows from Proposition 4.1. Indeed, it follows that

$$
\max _{\Sigma, R_{s}}(R) \cup \operatorname{dup}_{\Sigma, R_{s}}(R) \mid \leq\left(\begin{array}{c}
|r| \\
2
\end{array}\right) .
$$

Consequently, we have that

$$
\frac{\sqrt{1+8 \cdot\left|\max _{\Sigma, R_{s}}(R) \cup d u p_{\Sigma, R_{s}}(R)\right|}}{2} \leq|r| \text {. }
$$

The upper bound $\left|\max _{\Sigma, R_{s}}(R) \cup d u p_{\Sigma, R_{s}}(R)\right|+2$ follows immediately from Theorem 4.5 and Algorithm 4.3 .

We conclude that Algorithm 4.3 always computes an Armstrong table of reasonably small size.

Corollary 4.2. On input $\left(R, \Sigma, R_{s}\right)$, Algorithm 4.3 computes an Armstrong table for $\Sigma$ and $n f s\left(R_{s}\right)$ whose size is at most quadratic in the size of a minimumsized Armstrong tables for $\Sigma$ and $n f_{s}\left(R_{s}\right)$.

\subsubsection{The Size of Representations}

It is shown now that, in general, there is no most concise way of representing the information inherent in a set of weak uniqueness constraints, weak functional dependencies and null-free subschemata. We have already seen a case where the representation using Armstrong tables can be exponentially larger than the best equivalent constraint set, see Proposition 4.2 . 
Corollary 4.3. There is some WFD set $\Sigma$ and an NFS $n f s\left(R_{s}\right)$ such that $\Sigma$ has size $\mathcal{O}(n)$, and the size of a minimum-sized Armstrong table for $\Sigma$ and $n f_{s}\left(R_{s}\right)$ is $\mathcal{O}\left(2^{n / 2}\right)$.

The following theorem shows that in other cases, the representation using Armstrong tables can be exponentially smaller than the best representation using constraint sets. Extending Maier's notion of an optimal cover from relations [94], for a WFD set $\Sigma$ and an NFS $n f_{s}\left(R_{s}\right)$ we call a WFD set $\Sigma^{\prime}$ an optimal cover of $\Sigma$ with respect to $n f s\left(R_{s}\right)$ if

- $\Sigma^{\prime}$ is a cover of $\Sigma$ with respect to $n f_{s}\left(R_{s}\right)$, i.e., for every WFD $\sigma \in \Sigma$ we have $\Sigma^{\prime} \models{ }_{R_{s}} \sigma$; and for every WFD $\sigma^{\prime} \in \Sigma^{\prime}$ we have $\Sigma \models_{R_{s}} \sigma^{\prime}$; and

- there is no cover $\Sigma^{\prime \prime}$ of $\Sigma$ with respect to $n f s\left(R_{s}\right)$ such that $\Sigma^{\prime \prime}$ contains fewer symbol occurrences than $\Sigma^{\prime}$ (repeated symbol occurrences are counted as many times as they occur).

Theorem 4.6. There is some relation schema $R$, some NFS $n f_{s}\left(R_{s}\right)$ and some WFD set $\Sigma$ over $R$ such that there is an Armstrong table for $\Sigma$ and $n f_{s}\left(R_{s}\right)$ where the number of tuples is in $\mathcal{O}(n)$, and the optimal cover of $\Sigma$ with respect to $n f_{s}\left(R_{s}\right)$ has size $\mathcal{O}\left(2^{n}\right)$.

Proof. Let $R=A_{1} B_{1} \cdots A_{n} B_{n} C, R_{s}=R$ and

$$
\Sigma=\left\{\diamond\left(X_{1}, \ldots, X_{n} \rightarrow C\right) \mid \forall i=1, \ldots, n\left(X_{i} \in\left\{A_{i}, B_{i}\right\}\right)\right\} .
$$

We show that $\Sigma$ is the optimal cover of $\Sigma$ with respect to $n f_{S}\left(R_{s}\right)$, but there is an Armstrong table for $\Sigma$ and $n f_{s}\left(R_{s}\right)$ where the number of tuples is in $\mathcal{O}(n)$.

We will show first that $\Sigma$ is non-redundant (no subset of $\Sigma$ implies all WFDs in $\Sigma$ ), and then show that $\Sigma$ is an optimal cover of itself. We note that for every WFD $\sigma \in \Sigma$, where $X=L H S(\sigma)$ denotes the attribute set on the left-hand side of $\sigma$, the closure $X_{\Sigma-\{\sigma\}, R_{s}}^{*}$ of $X$ with respect to $\Sigma-\{\sigma\}$ and $n f s\left(R_{s}\right)$ is $X$ itself, i.e., $X_{\Sigma-\{\sigma\}, R_{s}}^{*}=X$. The reason is that there is no $\sigma^{\prime} \in \Sigma-\{\sigma\}$ such that $L H S\left(\sigma^{\prime}\right) \subseteq X$. Hence, $C \notin X_{\Sigma-\{\sigma\}, R_{s}}^{*}$ and we 
conclude that $\sigma$ is not implied by $\Sigma-\{\sigma\}$ and $n f_{s}\left(R_{s}\right)$. That is, $\Sigma$ is nonredundant.

Next we remark that every optimal cover $\Sigma^{\prime}$ of $\Sigma$ with respect to $n f s\left(R_{s}\right)$ contains only WFDs $\diamond(X \rightarrow Y)$ such that $Y=C$. Suppose, to the contrary, that there is some WFD $\diamond(X \rightarrow Y)$ in $\Sigma^{\prime}$ such that $Y \neq C$. If $Y-X=C$ and $Y \cap X \neq \emptyset$, then $\Sigma^{\prime}$ is not optimal since

$$
\left(\Sigma^{\prime}-\{\diamond(X \rightarrow Y)\}\right) \cup\{\diamond(X \rightarrow Y-X)\}
$$

is equivalent to $\Sigma$ but contains less attributes than $\Sigma^{\prime}$. If $Y-X=\emptyset$, then $\Sigma^{\prime}-\{\diamond(X \rightarrow Y)\}$ is equivalent to $\Sigma$ but contains less symbol occurrences than $\Sigma^{\prime}$. If $Y-X \neq \emptyset$ and $Y-X \neq C$, then $\Sigma \forall_{R_{s}} \diamond(X \rightarrow Y)$ and, therefore, $\Sigma^{\prime}$ is not a cover of $\Sigma$ with respect to $n f s\left(R_{s}\right)$. Moreover, every $\mathrm{WFD} \diamond(X \rightarrow Y)$ in an optimal cover $\Sigma^{\prime}$ of $\Sigma$ with respect to $n f_{s}\left(R_{s}\right)$ satisfies that $C \notin X$. If there was a WFD $\diamond(X \rightarrow C) \in \Sigma^{\prime}$ and $C \in X$, then

$$
\left(\Sigma^{\prime}-\{\diamond(X \rightarrow C)\}\right) \cup\{\diamond(X-C \rightarrow C)\}
$$

is equivalent to $\Sigma$ but contains less attributes than $\Sigma^{\prime}$.

Next we prove that there is no cover $\Sigma^{\prime}$ of $\Sigma$ with respect to $n f s\left(R_{s}\right)$ with a smaller number of attribute occurrences. Suppose there were an optimal cover $\Sigma^{\prime}$ of $\Sigma$ with respect to $n f_{s}\left(R_{s}\right)$ with a fewer number of attribute occurrences than $\Sigma$. Then for all $\sigma^{\prime}$ in $\Sigma^{\prime}$ it is the case that $\Sigma=_{R_{s}} \sigma^{\prime}$. Consequently, there must be some $\sigma \in \Sigma$ such that $L H S(\sigma) \subseteq L H S\left(\sigma^{\prime}\right)$. Suppose every WFD $\sigma \in \Sigma$ has the property that $L H S(\sigma) \subseteq L H S\left(\sigma^{\prime}\right)$ for a different WFD $\sigma^{\prime} \in \Sigma^{\prime}$. Then $\Sigma^{\prime}$ contains at least as many attribute occurrences as $\Sigma$, a contradiction. Otherwise, there is a proper subset $\Sigma^{\prime \prime}$ of $\Sigma$ such that every WFD $\sigma^{\prime} \in \Sigma^{\prime}$ has the property that $L H S(\sigma) \subseteq L H S\left(\sigma^{\prime}\right)$ for some $\sigma \in \Sigma^{\prime \prime}$. Consequently, $\Sigma^{\prime \prime}$ implies every WFD in $\Sigma^{\prime}$ with respect to $n f_{s}\left(R_{s}\right)$ and therefore also every WFD in $\Sigma$. This, however, is impossible since $\Sigma$ is non-redundant.

Thus we have just shown that $\Sigma$ is its own optimal cover with respect to $n f s\left(R_{s}\right)$, and thus exponential in the number of attributes. Now we show 
that there is an Armstrong table for $\Sigma$ and $n f s\left(R_{s}\right)$ where the number of tuples is in $\mathcal{O}(n)$. It suffices to show that the set $\max _{\Sigma, R_{s}}(R)$ contains a number of elements that is linear in the number of attributes. For each $i=1, \ldots, n$ we have $\max _{\Sigma, R_{s}}\left(A_{i}\right)=R-A_{i}$, and $\max _{\Sigma, R_{s}}\left(B_{i}\right)=R-B_{i}$. These are $2 n$ different maximal sets in total. The set $\max _{\Sigma, R_{s}}(C)$ consists of the following $n$ elements: $R-A_{i} B_{i} C, i=1, \ldots, n$. Therefore, $\max _{\Sigma, R_{s}}(R)$ has $3 n$ different elements. Since the set $\Sigma$ contains WFDs only, there is only one duplicate set $R$. Therefore, using Algorithm 4.3 we can easily create an Armstrong table for $\Sigma$ and $n f_{s}\left(R_{s}\right)$ that has $3 n+2$ tuples only. Indeed, as $R_{s}=R$, the null marker occurrences are not required, and, therefore, $3 n+1$ tuples suffice.

We can see that the representation in form of an Armstrong table can offer tremendous space savings over the representation as an abstract constraint set, and vice versa.

\subsection{Further Remarks}

In recent previous work Hartmann et al. investigated the combined class of uniqueness constraints and functional dependencies over SQL tables with null-free subschemata [64], which was reviewed in Chapter2. The semantics of WUCs and WFDs over Codd tables with null-free subschemata is fundamentally different from the semantics of uniqueness constraints and functional dependencies over SQL tables with null-free subschemata. Nevertheless, there are striking similarities between the results derived in this chapter and in previous work [64], respectively. For both classes the notions of weak and strong agree sets, of maximal sets and of duplicate sets are fundamental to characterize the structure of Armstrong tables. In both cases, the structural characterization leads to similar algorithms to compute Armstrong tables and the complexity results for the algorithms are very much similar, too. Finally, some remarks are in order to point 
out very important differences between the two combined classes. In the context of SQL tables, two tuples $t$ and $t^{\prime}$ weakly agree on an attribute $A$ if and only if $t(A)=\mathrm{ni}=t^{\prime}(A)$ [64]. In contrast, in the context of Codd tables, two tuples $t$ and $t^{\prime}$ weakly agree on an attribute $A$ if and only if $t(A)=$ unk or $t^{\prime}(A)=$ unk. In particular, the latter condition is in strong alignment with the weak possible world semantics, as weak agreement means that there is a possible world in which the tuples agree. While sets with non-standard functional dependencies over SQL tables with nullfree subschemata do not necessarily enjoy Armstrong tables [64], sets with arbitrary uniqueness constraints and functional dependencies, including non-standard ones, over Codd tables with null-free subschemata do always enjoy Armstrong tables. In essence, this difference is a result of the different semantics of non-standard WFDs and non-standard NFDs. Non-standard NFDs $\emptyset \rightarrow A$ permit only a constant value to appear in the column $A$ in SQL tables, and can therefore not be satisfied when $A$ must feature $\mathrm{n} i$ and some domain value. Non-standard WFDs can still be satisfied in such situations, due to the different notion of weak agreement in Codd tables. In the general construction of SQL tables that are Armstrong for a given set of standard uniqueness constraints and functional dependencies [64], each maximal and duplicate set results in two new tuples that are added to the Armstrong table. In contrast, in the general construction of Codd tables that are Armstrong for a given set of weak uniqueness constraints and functional dependencies, each maximal and duplicate set results in a single new tuple that is added to the Armstrong table. Therefore, Armstrong tables generated for some sets of weak uniqueness constraints and functional dependencies have roughly half the number of rows as Armstrong tables generated for the corresponding sets of SQL uniqueness constraints and functional dependencies. Note, however, that this is not true for every set.

Finally, Armstrong tables were also investigated for the sole class of uniqueness constraints over SQL tables with null-free subschemata 83 . 
84]. Here, the role of maximal and duplicate sets is replaced by the notion of an anti-key. As one would expect intuitively, the focus on a less expressive class of constraints results in smaller sizes of Armstrong tables for them. Therefore, if designers decide to not address functional dependencies or the schemata are in Boyce-Codd normal form, then they can utilize smaller Armstrong tables to communicate and validate their understanding of the semantics of the application domain.

\subsection{Summary}

In this chapter structural and computational properties of Armstrong tables were established for the combined class of weak uniqueness constraints and weak functional dependencies over Codd tables with null-free subschemata. It was shown that the entire class does enjoy Armstrong tables. Sufficient and necessary conditions were established for an arbitrarily given Codd table to be Armstrong for an arbitrarily given set of constraints in the combined class. Based on this structural characterization an algorithm for computing an Armstrong table for an arbitrarily given set of constraints in the combined class was established. The problem of finding such an Armstrong table remains precisely exponential in the number of the underlying relation schema, as for the special case of Armstrong relations. The algorithm always produces Armstrong tables whose number of rows is guaranteed to be at most quadratic in the number of rows in a minimum-sized Armstrong table. Finally, there are cases where the number of rows in a minimum-sized Armstrong table is exponential in the size of the given constraints, but there are also cases where the number of rows in an Armstrong table is logarithmic in the size of the optimal cover of a given constraint set. The results of this chapter were announced in [56], and similar results have been obtained for the sole class of uniqueness constraints over SQL tables with null-free subschemata [83, 84]. 


\section{Chapter 5}

\section{Implementation: SQL-Sampler}

This chapter addresses the second objective of this thesis, stated at the end of Chapter 2. The second main contribution of this thesis is the first prototype of a toolbox, called SQL-Sampler, for computing Armstrong tables with respect to different classes of constraints and different interpretations of null marker occurrences. This chapter is organized as follows. In Section 5.1 an introduction to the functionality of SQL-Sampler is given, together with some screenshots of its graphical user interface. A brief outline of the system requirements for SQL-Sampler is given in Section 5.2. The modules that constitute the overall design for the user interface of SQLSampler are presented in Section 5.3. Subsequently, Section 5.4 describes in detail which of the algorithms from Chapter 3 and Chapter 4 are linked in which way to which of the modules in SQL-Sampler. The use of SQLSampler is illustrated by several screenshots of our running example in Section 5.5. Finally, Section 5.6 contains a brief summary of this Chapter. $S Q L-S a m p l e r$ was introduced and demonstrated to the research community based on a conference paper [82]. 


\subsection{Introduction to SQL-Sampler}

SQL-Sampler is the first toolbox to implement new as well as recently developed algorithms to compute Armstrong tables for different classes of constraints under different interpretations of null marker occurrences. Its primary purpose is to assist data engineers in their task of identifying constraints that are semantically meaningful to a given application domain. The engineers can apply the tool to generate concise data samples that perfectly visualize their current understanding of the semantics of the application domain. They can analyze the data samples to consolidate their understanding, either by themselves, or as a basis for discussing semantic requirements with domain experts. The Armstrong tables can also be exploited to transform a given relational approximation of the target database into a real SQL design. Leading database design tools, such as ERWin, promote the creation of test data to validate, communicate and consolidate the data models they produce [22].

SQL-Sampler is available as a desktop version and as a Web-based version. An installation package of the desktop version can be downloaded at

http://armstrongtable.sim.vuw.ac.nz/ArmstrongData.zip

Figure 5.1 shows the main user interface of SQL-Sampler in the desktop version under the Windows operating system.

The Web-based version of SQL-Sampler is available at

https://armstrongtable.sim.vuw.ac.nz.

Figure 5.2 shows a screenshot of its user interface. Both versions have slightly different designs, but offer the same functionality. 


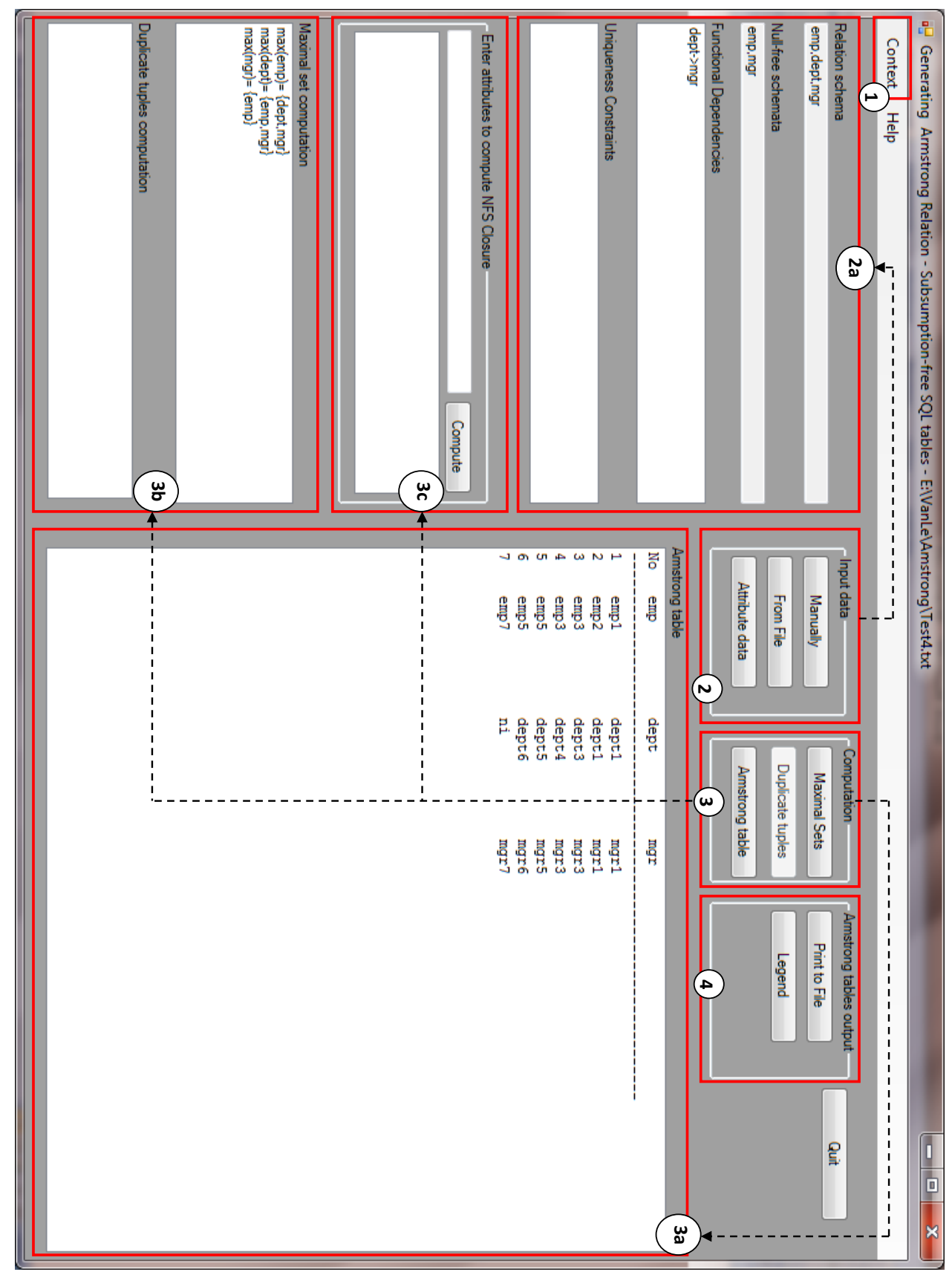

Figure 5.1: Main interface of SQL-Sampler's desktop version. Users operate the application through components of: -1) Context menu -2) Input data buttons - 3) Computation button - 4) Armstrong tables output button. Display: 2a) Area to input data - 3a), 3b), 3c) Areas to output Armstrong table and the underlying features 


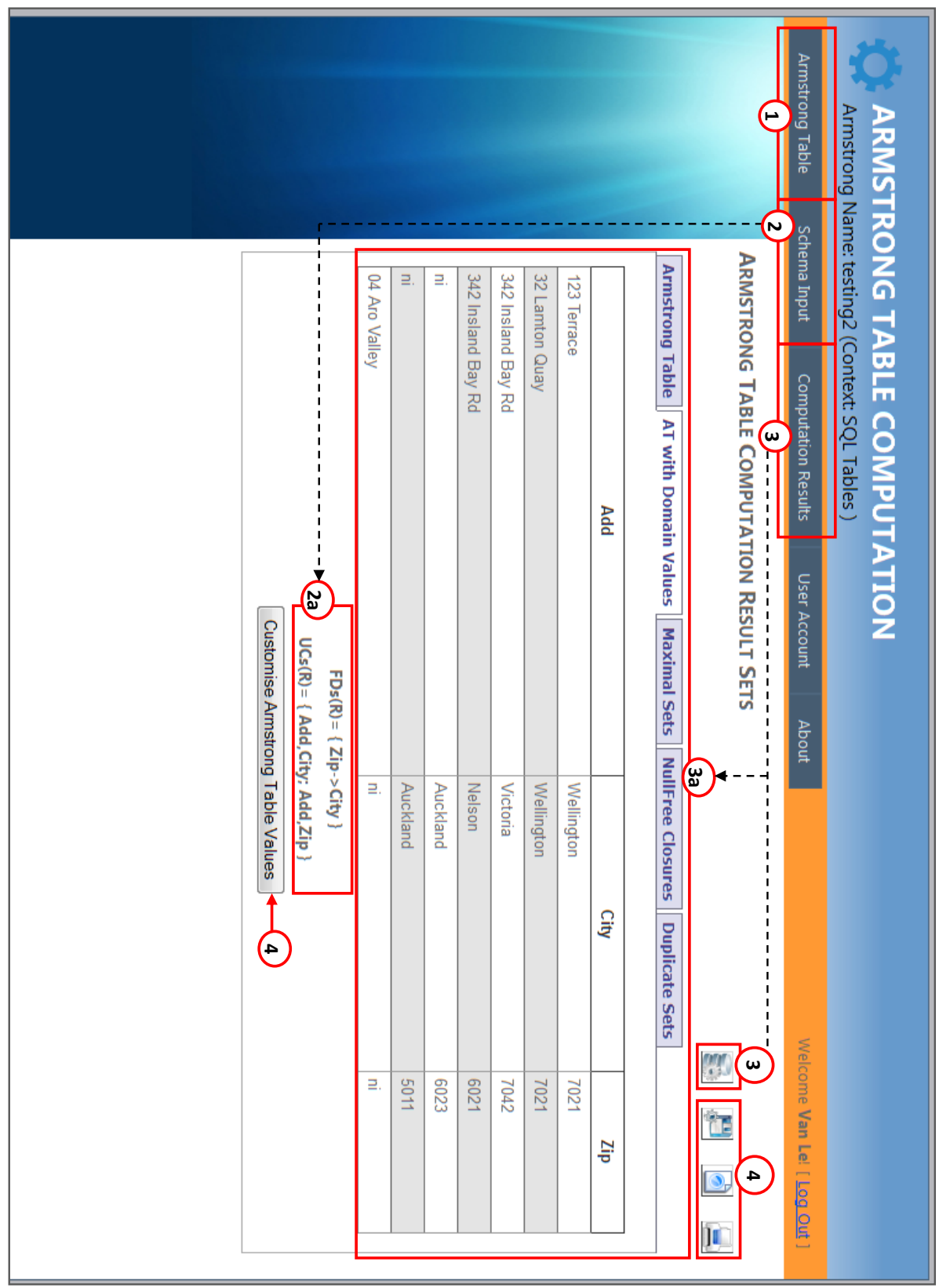

Figure 5.2: Main interface of SQL-Sampler's Web-based version. Users operate the application through components of: -1) Context menu - 2) Schema Input menu - 3) Computation Results menu or Compute Armstrong table button-4) Output data buttons. Display: 2a) Area to input constraints - 3a) Area to output Armstrong table and underlying features 


\subsection{System Requirements}

The desktop version of SQL-Sampler operates on a 32-bit Windows system and the .NET framework 3.5 [44].

The Web-based application of SQL-Sampler requires a common Internet browser such as Firefox, Internet Explorer, or Google Chrome on the client side. At the server-side, SQL-Sampler requires Internet Information Services (IIS) [110] and ASP.NET Framework 4.0 [43], as well as SQL server $2005 / 2008$ [57].

\subsection{Design}

The basic design of SQL-Sampler is based on four different modules that are built on top of a database. The design and workflow of the modules is illustrated in Figure 5.3. The modules consist of

- the Context Definition,

- the Input Definition,

- the Computation, and

- the Output.

In what follows we briefly describe the functionality of each module.

\subsubsection{DEFINE CONTEXT module}

This module provides users with the ability to define the context in which Armstrong tables and related features are computed subsequently. Users define a context by selecting i) an interpretation of null marker occurrences, that is, either the "no information" interpretation ni or the "value 


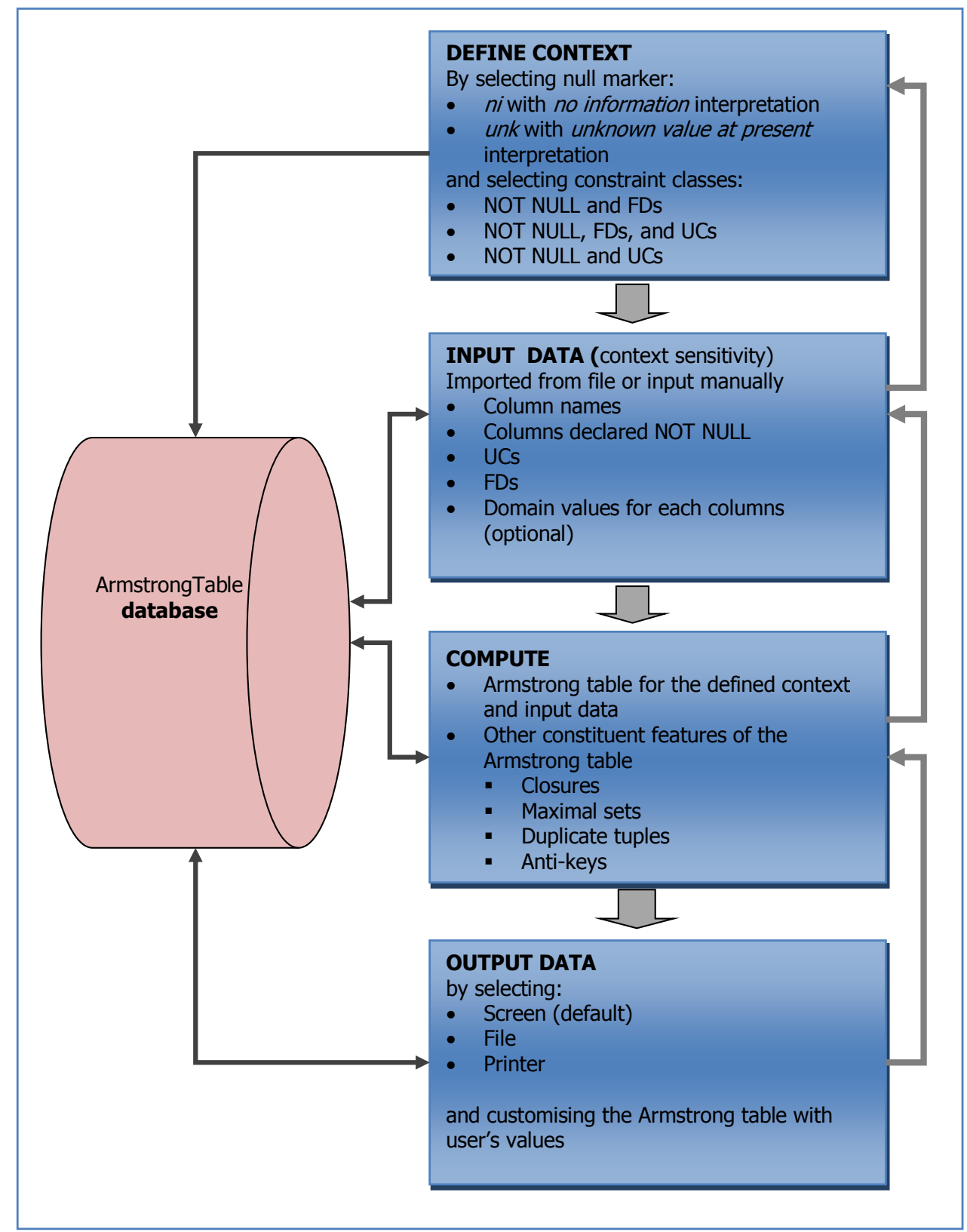

Figure 5.3: Design of SQL-Sampler with four modules and a database 
at present unknown" interpretation unk, and ii) a class $\mathcal{C}$ for which $\mathcal{C}$ Armstrong tables will be computed. Under the "no information" interpretation, users can select one of the three following classes: a) uniqueness constraints with nulls, b) functional dependencies with nulls, or c) uniqueness constraints with nulls and functional dependencies with nulls. Under the "value at present unknown" interpretation, users can compute Armstrong tables with respect to the class of d) weak uniqueness constraints and weak functional dependencies. All classes of constraints include a null-free subschema, that is, the ability to specify attributes as NOT NULL.

In the user interface, users define the context by choosing one of the following options, as illustrated in Figure 5.4 .

a) SQL table for keys: This option computes SQL tables that are Armstrong with respect to the class of uniqueness constraints with nulls (NUCs) and NOT NULL constraints. The implementation of the algorithms is based on those published in [83, 84].

b) Subsumption-free SQL table: This option computes subsumptionfree SQL tables that are Armstrong with respect to the class of functional dependencies with nulls (NFDs) and NOT NULL constraints. Recall that in the absence of subsumed tuples, the class of uniqueness constraints with nulls can be expressed by the class of functional dependencies with nulls. The implementation of the algorithms is based on those published in [64, 91].

c) SQL table: This option computes SQL tables that are Armstrong with respect to the class of uniqueness constraints with nulls (NUCs), functional dependencies with nulls (NFDs) and NOT NULL constraints. Recall that in the presence of subsumed tuples, the class of uniqueness constraints with nulls cannot be expressed by the class of functional dependencies with nulls. The implementation of the algorithms is based on those published in [64, 91]. 
d) Codd table: This option computes Codd tables that are Armstrong with respect to the class of weak uniqueness constraints (WUCs), weak functional dependencies (WFDs) and NOT NULL constraints. The implementation of the algorithms is based on the algorithms from Chapter 3 and Chapter 4 , also announced in [56].

\subsubsection{INPUT DATA module}

This module allows users of SQL-Sampler to input the data that can be used to compute an Armstrong table with respect to the context they have previously defined. This input data includes: i) the attributes of the underlying relation schema, ii) the attributes declared NOT NULL to define the null-free subschema, iii) the set of uniqueness constraints, iv) the set of functional dependencies, and v) domain values of attributes that can be used to populate the Armstrong table that is computed. Figure 5.2 shows an example of an Armstrong table that is populated by domain values previously entered by users during the Input data stage. If the user does not provide domain values, the Armstrong tables are populated with generic values, as shown in Figure 5.1 .

The data that is used by the algorithms depends on the context that has been selected by the users. Figure 5.5 shows which data can be used in which context. For example, in the context of Subsumption-free SQL tables no uniqueness constraints with nulls can be declared. Similarly, in the context of SQL tables for keys no functional dependencies with nulls can be declared.

\subsubsection{COMPUTE module}

This module contains the algorithms that compute a $\mathcal{C}$-Armstrong table for the set $\Sigma$ of constraints over relation schema $R$ with null-free subschema 


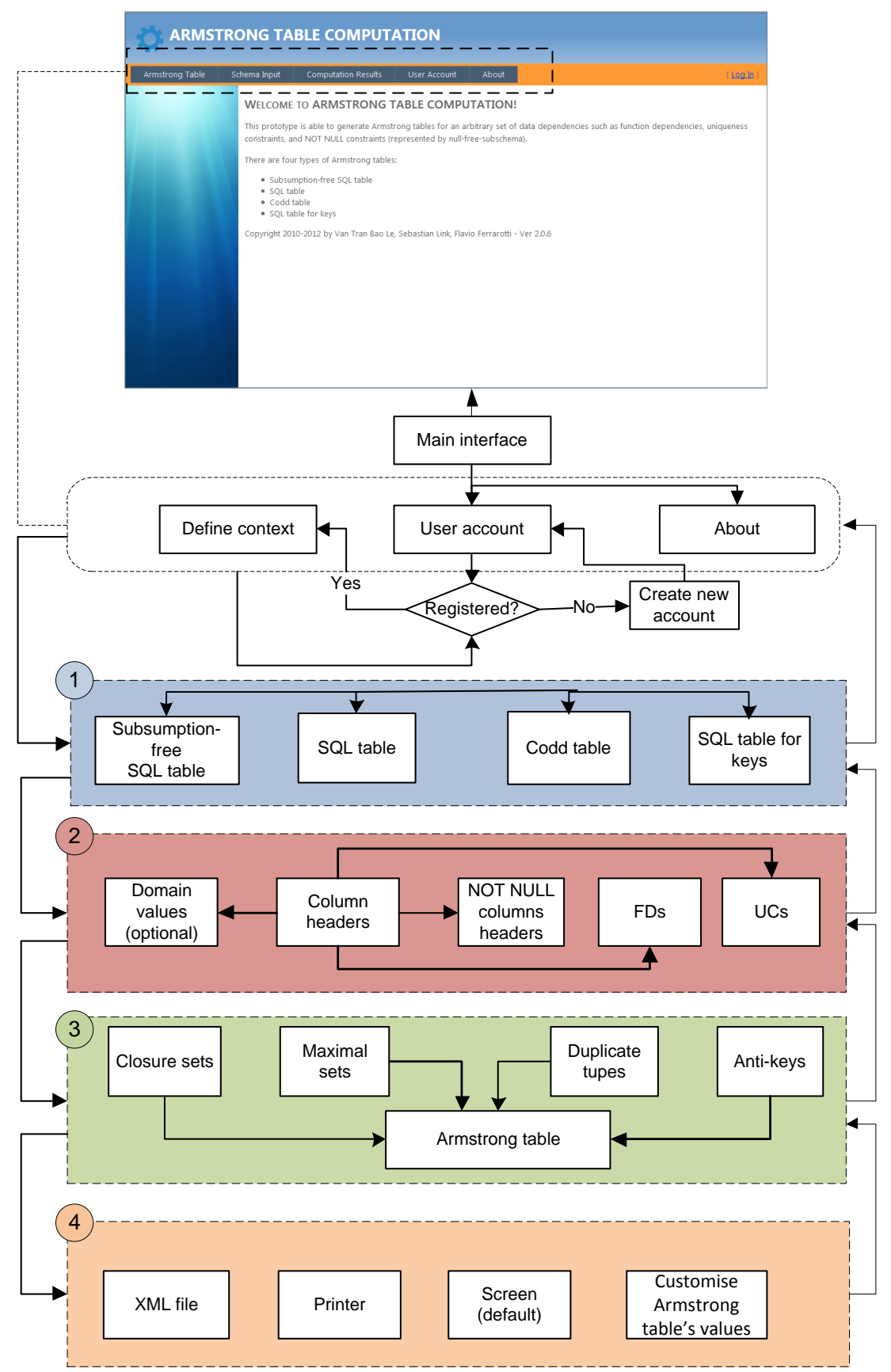

Figure 5.4: Work flow of SQL-Sampler for four modules (in numbered blocks) and objectives of each module (text in rectangle box). 1) Context Defining module- 2) Data Input module- 3) Computation module- 4) Data Output module 


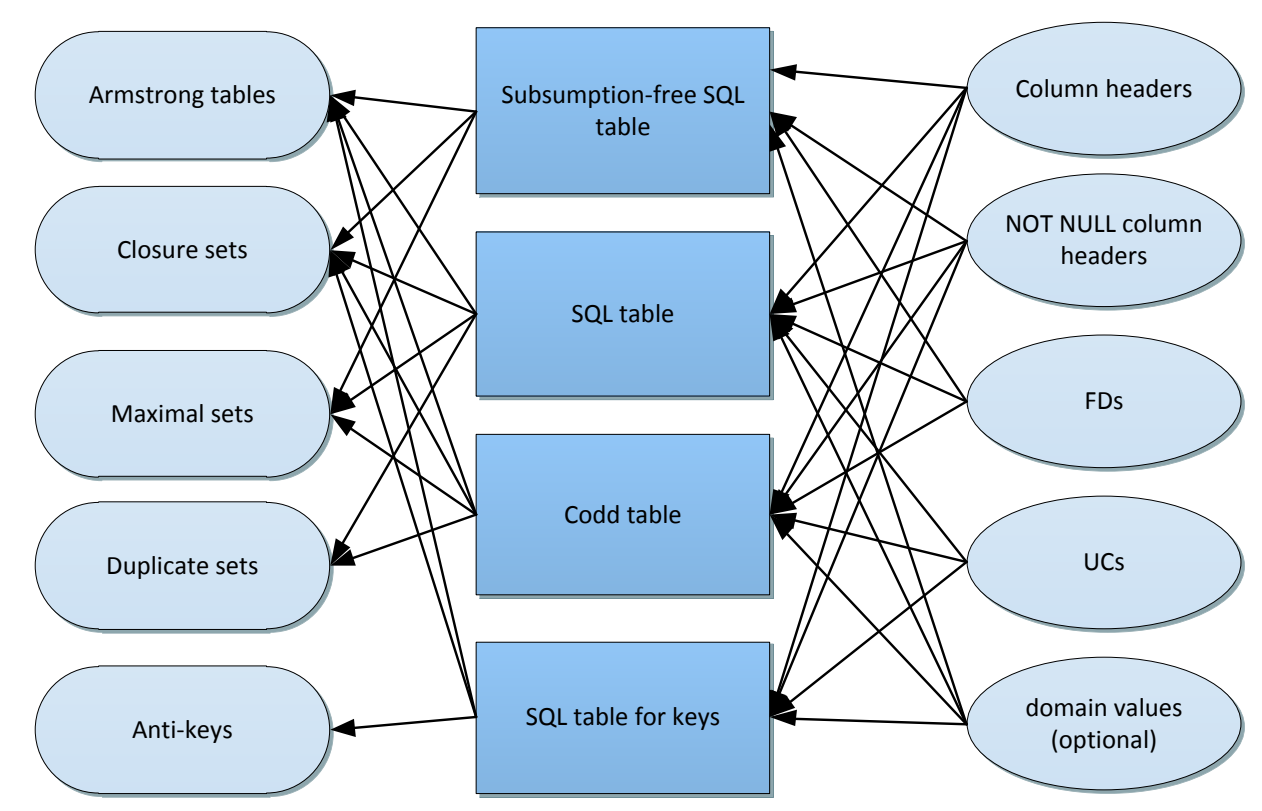

Figure 5.5: Data input (text in ellipses) and output (text in rounded squares) for each context (text in rectangle)

$n f_{s}\left(R_{s}\right)$, provided by the user in the data input, with respect to the context $\mathcal{C}$, that the user selected during the context definition. The algorithms differ from context to context.

The module also contains algorithms that show several features of the produced table. These include attribute set closures, maximal sets, duplicate sets, and anti-keys, for example. Figure 5.5 shows which features are available in which context. In the context Subsumption-free SQL table, for example, duplicate sets are not required to be computed. Their computation, however, is necessary in the contexts of SQL tables and Codd tables. The main purpose of the features is educational: they enable students or designers to better comprehend the construction of the tables produced. 


\subsubsection{OUTPUT DATA module}

SQL-Sampler provides three options to display the output it produces: i) screen, ii) printer, or iii) file. Apart from displaying the output on screen as default, SQL-Sampler can also export the output to an XML file or to a printer for subsequent use. The XML format is supported because it can be processed easily by several common applications (for example, Microsoft Office, OpenOffice, Apple's iWork, etc.) and allows the output data to be exchanged easily.

SQL-Sampler gives its users control over the data values that populate an Armstrong table. The user may choose to input domain values for attributes during the Input data stage, which are then used to populate subsequent Armstrong tables, cf. Figure 5.2. If the set of supplied domain values is insufficient, then the algorithms create additional generic data values on the fly. For example, the Armstrong table in Figure 5.1 has been entirely populated by generic data values. Users may also choose to substitute any generic data values by any values that they prefer to use. This, however, is done in such a way that the resulting table remains Armstrong. Figure 5.2 shows the option to Customise Armstrong Table Values.

\subsubsection{Armstrongtable database}

The Web-based version of SQL-Sampler maintains a database, called ARMSTRONGTABLE, to manage all the data necessary to produce the output with the help of the prototype. The conceptual diagram of the database is shown in Figure 5.6. Next, we explain the function of each table in the ARMSTRONGTABLE database.

- ArmstrongTables(ArmsID, ArmsName, ContID, CreateUser, CreateDate, LastUpdate, Notes): This table contains the name, ID, and user-related information for each Armstrong table. Most informa- 


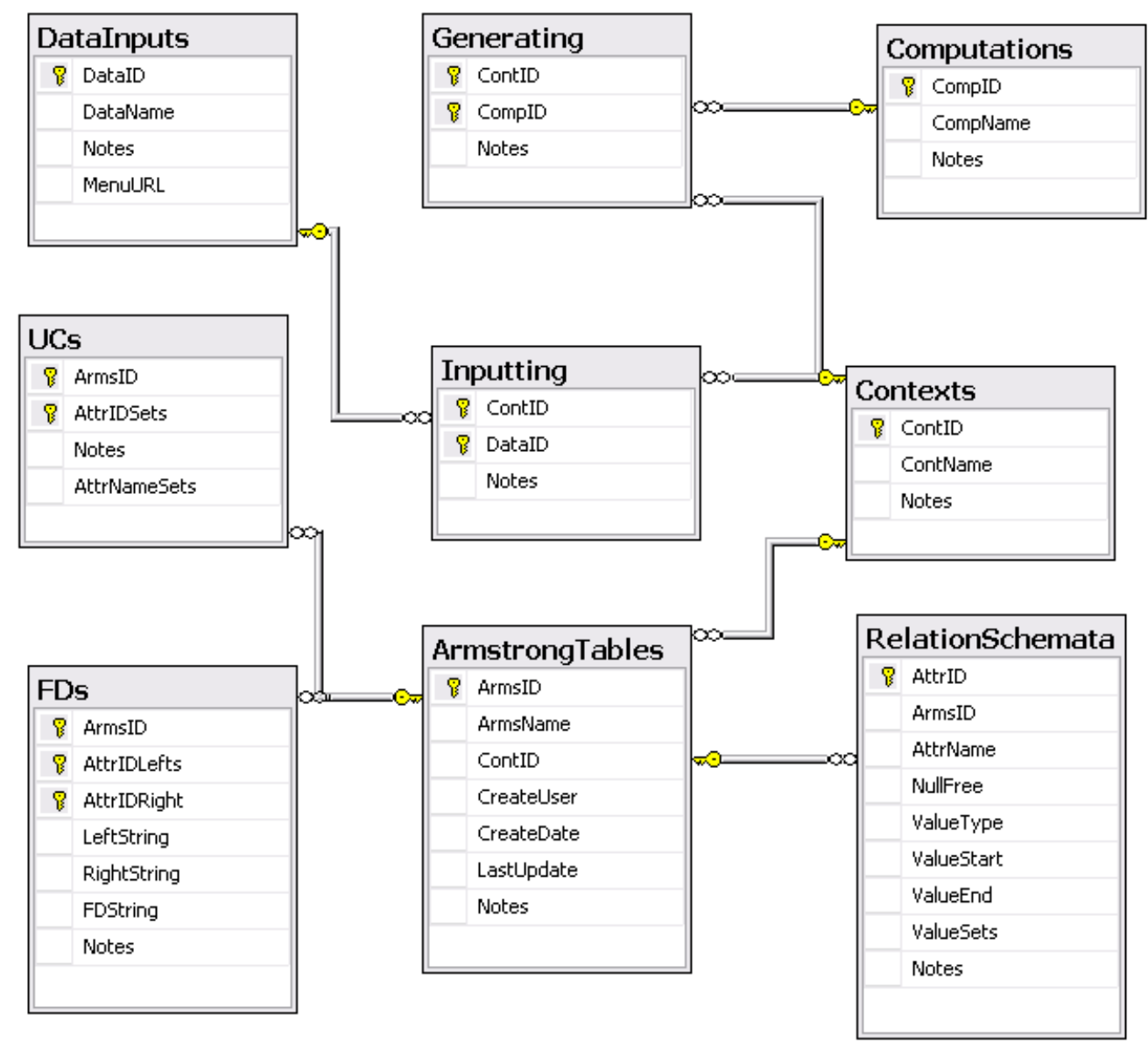

Figure 5.6: Conceptual Diagram of Back-end Database for SQL-Sampler 
tion in this table is automatically updated except for the name of the Armstrong table which is provided by the user.

- RelationSchemata( $\underline{\text { AttrID }}$ ArmsID, AttrName, NullFree, ValueType, ValueStart, ValueEnd, ValueSets, Notes): It stores properties of attributes including their name, domain type, null-free value, start value and end value which define a range of automatic data values to populate an Armstrong table. If users specify their own domain values, the values will be stored in the ValueSets column.

- FDs(ArmsID, AttrIDLefts, AttRight, LeftString, RightString, FDString, Notes): It contains the functional dependencies the user specifies during the input data stage.

- UCs(ArmsID, AttrIDSets, AttrNameSets, Notes): It contains the uniqueness constraints the user specifies during the input data stage.

- DataInputs(DataID, DataName, Notes, MenuURL): It contains the categories of input data which SQL-Sampler assembles to generate each type of Armstrong table. Currently, this table consists of four rows to encode names of attributes, functional dependencies, uniqueness constraints, and domain values as input data categories. Data in this table cannot be updated by users.

- Contexts(ContID, ContName, Notes): It stores the different contexts in which Armstrong tables can be computed by SQL-Sampler. Currently, this table contains four rows to encode subsumption-free SQL tables, SQL tables, Codd tables, and SQL tables for keys, as previously described. Data in this table cannot be updated by users.

- Inputting(ContID, DataID, Notes): This table specifies the inputs for each context in which Armstrong tables can be computed. For example, this table has four rows to specify the names of attributes, nullfree subschema, functional dependencies with nulls, and unique- 
ness constraints with nulls as input for the context $S Q L$ table; and it has three rows to specify the names of attributes, null-free subschema, and functional dependencies with nulls as input for the context Subsumption-free SQL table. More details on the input data for each context is shown in Figure 5.5. Data in this table cannot be updated by users.

- Computations(CompID,CompName,Notes): It contains the possible types of outputs computed by SQL-Sampler. Currently, this table consists of fives rows to encode Armstrong tables, closures of attribute subsets, maximal sets, duplicate sets, and anti-keys as possible output categories for SQL-Sampler. Data in this table cannot be updated by users.

- Generating(ContID, CompID, Notes): This table specifies the types of outputs available in each given context of SQL-Sampler. Figure 5.5 shows the different types available in each context. In the context Codd table, for example, output is available as Armstrong tables, attribute set closures, maximal sets, and duplicate sets. Data in this table cannot be updated by users.

\subsection{Implementation Details}

In this section some implementation details are given on how SQL-Sampler actually computes Armstrong tables for the specified input data with respect to the specified context. In the following we describe the implementation of the four modules Define Context, Data Input, Computation, and Output. 


\subsubsection{Context variable}

During Context Definition a user specifies the context $\mathcal{C}$ in which Armstrong tables will subsequently be computed. In the implementation of SQLSampler a global variable, called contextChosen, is given a value to identify one of the four contexts available. The following table shows which value of contextChosen corresponds to which context.

\begin{tabular}{cc}
\hline ContextChosen value & Context \\
\hline 1 & Subsumption-free SQL table \\
2 & SQL table \\
3 & Codd table \\
4 & SQL table for keys \\
\hline
\end{tabular}

\subsubsection{DATA INPUT functions}

SQL-Sampler provides users with two options to input data: i) entering data manually, or ii) entering data from a file. The following functions carry out the task of retrieving user input data. The functions produce the names of attributes, the names of attributes declared NOT NULL, functional dependencies, and uniqueness constraints, as illustrated in Figure 5.7

- GetAttributeList(out AttList, out NFSList, out DomainList) function: returns the names of attributes, and the names of attributes declared NOT NULL.

- GetFunctionalDependencyList(out FDList) function: returns a list of functional dependencies.

- GetUniquenessConstraintList(ArmsID, out UCList) function: returns a list of uniqueness constraints. 
The use of the functions is described in more detail in the following subsections.

\subsubsection{COMPUTATION functions}

Next we describe how SQL-Sampler implements the module Compute in order to generate Armstrong tables with respect to different contexts.

\section{ArmstrongTableComputation $(C)$ function}

This function controls how SQL-Sampler selects the various algorithms for computing Armstrong tables, based on the selected context. Next, we denote by $C$ the global variable contextChosen.

Basically, SQL-Sampler uses the value of $C$ to select the corresponding algorithms as follows:

- if the value of $C$ is 1 , an SQL table is computed that is Armstrong for the given input data with respect to the context Subsumption-free $S Q L$ table. This is achieved by a call of the function

\section{ComputeArmstrongRelation SubsumptionFreeSQLTable(AttList, NFSList, $\Sigma$ ).}

Input data for this function includes a list AttList of attributes, a list NFSList of attributes declared NOT NULL, and a set $\Sigma$ of functional dependencies with nulls (NFDs). The algorithm that produces the output Armstrong table is given in [64,91].

- if the value of $C$ is 2, an SQL table is computed that is Armstrong for the given input data with respect to the context $S Q L$ table. This is achieved by a call of the function 


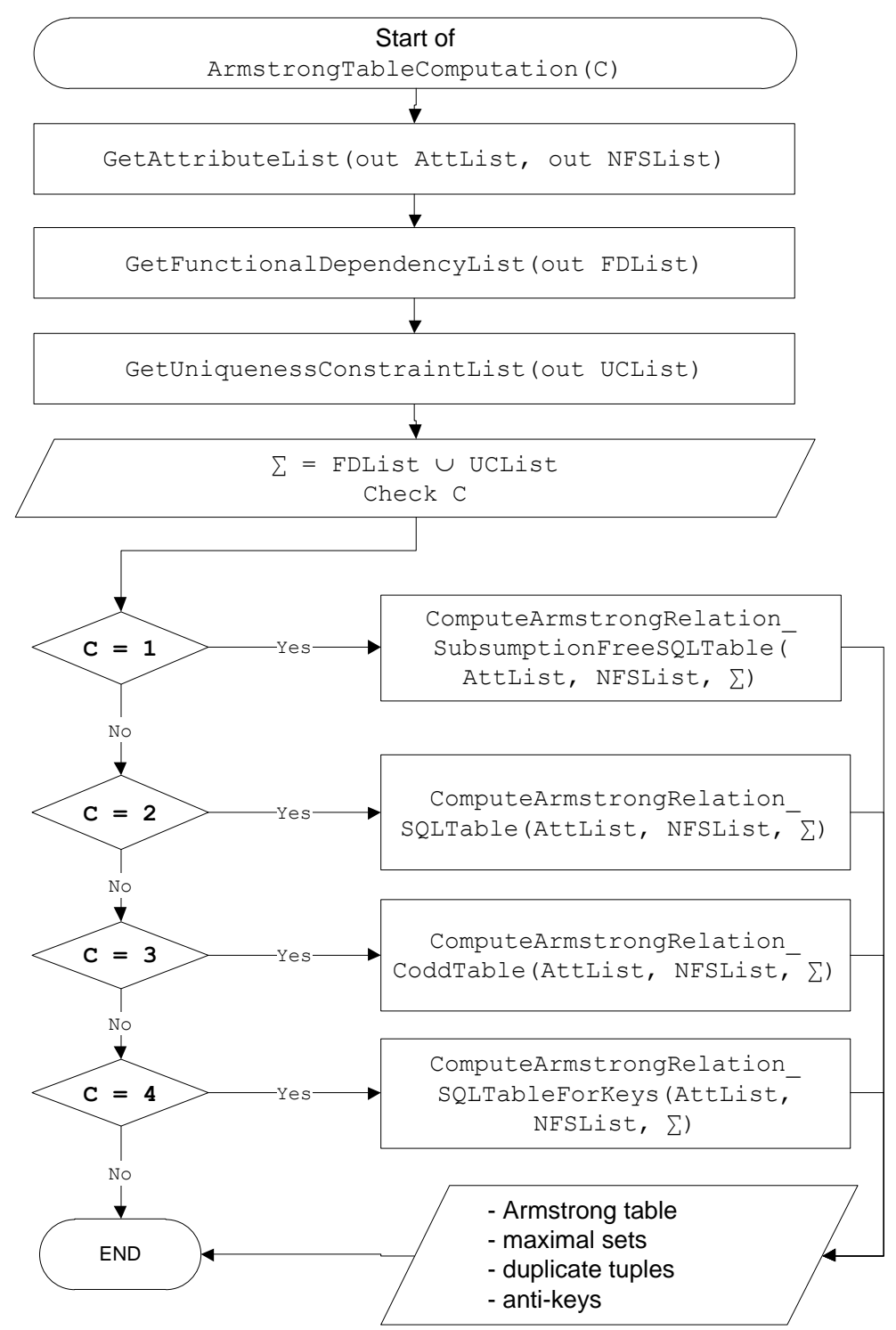

Figure 5.7: The flow of functions (in the rectangular box) implemented to compute Armstrong tables for different classes of constraints and different interpretations of null marker occurrences 
Input data for this function includes a list AttList of attributes, a list NFSList of attributes declared NOT NULL, and a set $\Sigma$ of uniqueness constraints with nulls (NUCs) and functional dependencies with nulls (NFDs). The algorithm that produces the output Armstrong table is given in 64,91$]$.

- if the value of $C$ is 3, a Codd table is computed that is Armstrong for the given input data with respect to the context Codd table. This is achieved by a call of the function

ComputeArmstrongRelation_CoddTable(AttList, NFSList, $\Sigma)$.

Input data for this function includes a list AttList of attributes, a list NFSList of attributes declared NOT NULL, and a set $\Sigma$ of weak uniqueness constraints and weak functional dependencies. The algorithm that produces the output Armstrong table is given in Chapter 4 and was announced in [56].

- if the value of $C$ is 4, an SQL table is computed that is Armstrong for the given input data with respect to the context $S Q L$ table for Keys. This is achieved by a call of the function

ComputeArmstrongRelation_SQLTableForKeys(AttList, NFSList, $\Sigma)$.

Input data for this function includes a list AttList of attributes, a list NFSList of attributes declared NOT NULL, and a set $\Sigma$ of uniqueness constraints with nulls (NUCs). The algorithm that produces the output Armstrong table is given in [83,84].

Next we comment briefly on the implementation of central algorithms for the computation of Armstrong tables. These include the computation of the maximal set families, the computation of duplicate sets, and the computation of anti-keys. 
MaximalSetsComputation(AttList, NFSList, $\Sigma$, out maxSetList) function

This function takes as input a list AttList of attribute names, a list NFSList of attributes declared NOT NULL, and a set $\Sigma$ of functional dependencies. The output of the function is a list of maximal sets. Figure 5.8 shows details related to the computation of maximal sets. Note that in the context of SQL tables, empty attribute sets are discarded from the set of maximal sets, while in the context of Codd tables, empty attribute sets can be maximal. The implementation of this function is based on Algorithm 2.7 and Algorithm 4.1.

For example, with the AttList $=\{$ Date, Emp, Proj, Hrs, Role $\}$, NFSList $=$ $\{$ Date, Emp $\}$ and a set $\Sigma=\{$ Emp, Proj $\rightarrow$ Hrs;Emp, Proj $\rightarrow$ Role; Proj, Role $\rightarrow$ $H r s ; u(D a t e, E m p)\}$, the output resulting from this function is:

- $\operatorname{maxset}($ Date $)=\{$ Emp,Hrs,Proj,Role $\}$

- $\operatorname{maxset}(\mathrm{Emp})=\{$ Date,Hrs,Proj,Role $\}$

- $\operatorname{maxset}(\mathrm{Hrs})=\{\{$ Date, Role $\},\{$ Date,Proj $\},\{$ Emp, Role $\}\}$

- $\operatorname{maxset}($ Proj $)=\{\{$ Emp,Hrs,Role $\},\{$ Date,Hrs,Role $\}\}$

- $\operatorname{maxset}($ Role $)=\{\{$ Date,Hrs,Proj $\},\{$ Emp,Hrs $\}\}$

\section{Function}

\section{DuplicateSetsComputation(AttList, NFSList, $\Sigma$, out dupTupleList)}

As shown in Figure 5.8 the computation of duplicate sets is important for the computation of Armstrong tables in the contexts of Codd tables and SQL tables, respectively. The function

DuplicateTuplesComputation(AttList, NFSList, $\Sigma$ ) 


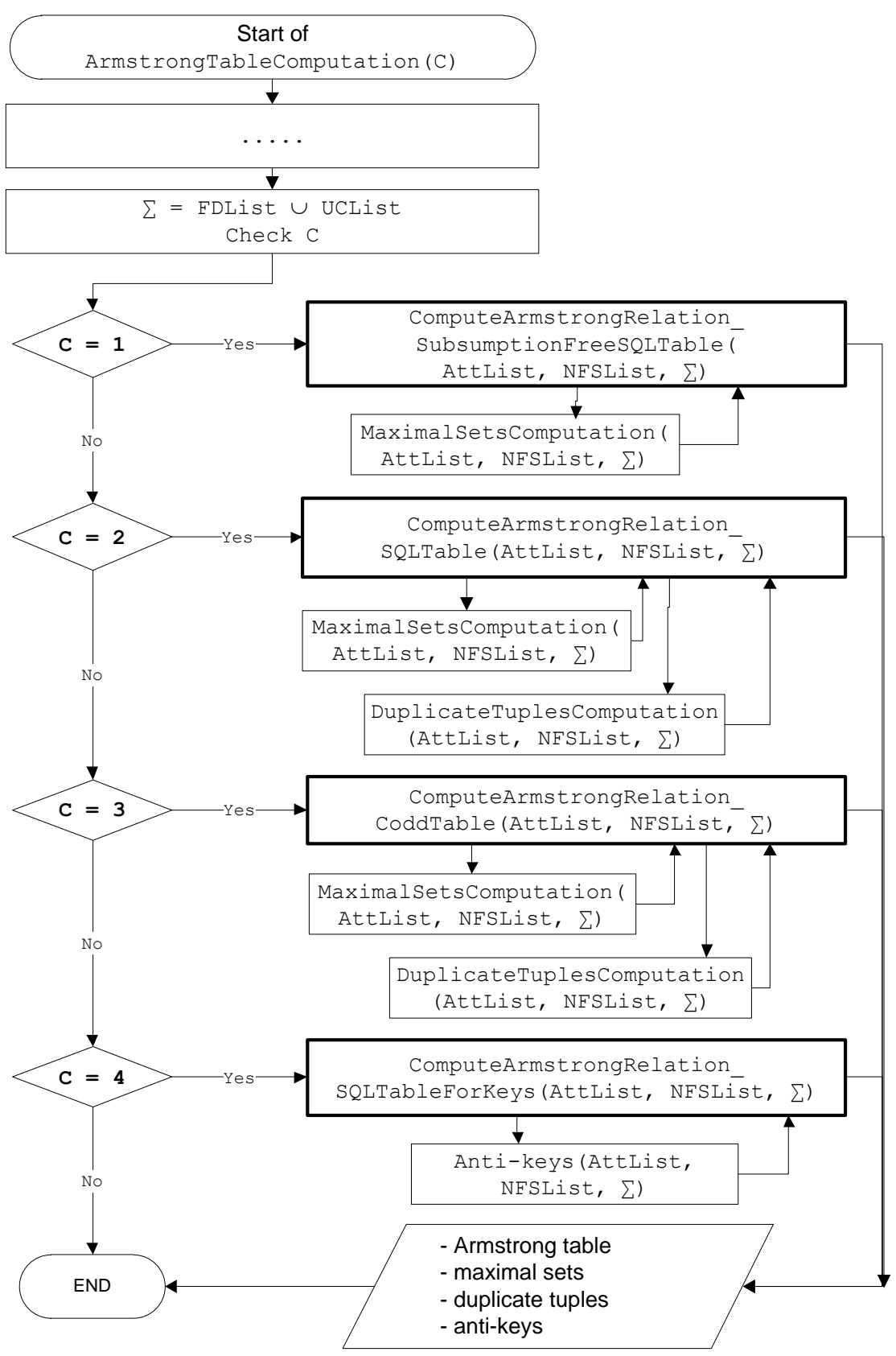

Figure 5.8: Work flow for the computations of maximal sets, duplicate sets, and anti-keys (in the rectangular box) in each context for producing Armstrong table 
requires a list AttList of attribute names, a list NFSList of names for attributes declared NOT NULL, and a set $\Sigma$ of uniqueness constraints and functional dependencies. The function returns the list of duplicate sets for $\Sigma$ and NFSList. For example, with AttList $=\{$ Date, Emp, Proj, Hrs, Role $\},$ NFSList $=\{$ Date, Emp $\}$ and a set $\Sigma=\{$ Emp, Proj $\rightarrow$ Hrs; Emp, Proj $\rightarrow$ Role; Proj, Role $\rightarrow \mathrm{Hrs} ; \mathrm{u}($ Date,Emp) $\}$, the output resulting from this function is the list dupSetList that contains the empty attribute set only.

\section{AntikeysComputation(AttList, NFSList, $\Sigma$, out AntiKeyList) function}

As shown in Figure 5.8 the computation of anti-keys is important for the computation of Armstrong tables in the context of SQL tables with Keys. The function

\section{AntikeysComputation(AttList, NFSList, $\Sigma$ )}

requires a list AttList of attribute names, a list NFSList of names for attributes declared NOT NULL, and a set $\Sigma$ of uniqueness constraints with nulls. The function returns the list of anti-keys for $\Sigma$ and NFSList. For example, with AttList $=\{$ Date, Emp, Proj, Hrs, Role $\}$, NFSList $=\{$ Date, Emp $\}$ and a set $\Sigma=\{u($ Date,Emp $)\}$, the output resulting from this function is the list AntiKeysList that contains the anti-keys $\{\mathrm{Hrs}$, Proj,Role,Date $\}$ and $\{$ Hrs,Proj,Role,Emp $\}$.

\subsubsection{DATA OUTPUT functions}

As indicated before, SQL-Sampler provides three different ways to display an Armstrong table and its features. The three options to output this data include the screen by default, a file, and a printer. Each of these options has been implemented by its own function.

\section{Screen output}

The function 


\section{PrintArmstrongTable(ArmsTable, AttList, NFSList, DomainList)}

displays the computed Armstrong table on screen. The data of this table are already stored in the array ArmsTable. Attribute names of the table are stored in AttList, the attributes declared NOT NULL are recorded in NFSList, and the domain values for each attribute are stored in DomainList.

The function

\section{PrintOut(List)}

displays the list List on screen. The list may contain duplicate sets, maximal sets, or anti-keys. This function, therefore, displays the features of Armstrong tables on screen.

\section{File output}

The function

\section{PrintToFile()}

exports the computed Armstrong table and its features, which are already displayed on screen, to a file. SQL-Sampler generates a .txt file in the desktop version, and a .xml file in the web-based version.

\section{Printer output}

The function

\section{PrintGrids()}

exports the computed Armstrong table and its features, which are already displayed on screen, to a printer. This function was developed as a JavaScript, which is more time efficient than a server side script. All computed results are already in the browser and JavaScript is the most effective language to extend functionality of a website on the client side. 


\subsection{Use Case Example}

In this section the use of SQL-Sampler is illustrated on one of our running examples. First we describe the input to the use case, followed by screen shots that show the use of SQL-Sampler in computing an Armstrong table for the input.

\subsubsection{The Use Case}

As use case we select the relation schema CONTACT which consists of the attributes Address, City, and ZIP. The null-free subschema $n f_{s}\left(\mathrm{CONTACT}_{s}\right)$ is defined by $\mathrm{CONTACT}_{s}=\{Z I P\}$, and as the input set of constraints we select the set

$$
\Sigma=\{\diamond \mathfrak{u}(\text { Address }, \text { City }), \diamond(Z I P \rightarrow \text { City })\}
$$

that consists of a weak uniqueness constraint and a weak functional dependency. We illustrate how SQL-Sampler can be used to compute an Armstrong table for $\Sigma$ and $n f s\left(\mathrm{CONTACT}_{s}\right)$ with respect to weak uniqueness constraints, weak functional dependencies and NOT NULL constraints.

\subsubsection{Context}

The use case description above tells us which context needs to be defined: We select the context Codd Table, which means that the interpretation of all null marker occurrences unk in the Codd table are fixed to "value unknown at present". The Armstrong table is computed with respect to the combined class of WUCs, WFDs with NOT NULL constraints. Figure 5.9 shows how the context can easily be selected in SQL-Sampler.

\subsubsection{Input Data}

Figure 5.10 shows a screenshot of the Input Data module of SQL-Sampler after the data from the use case was filled in. 


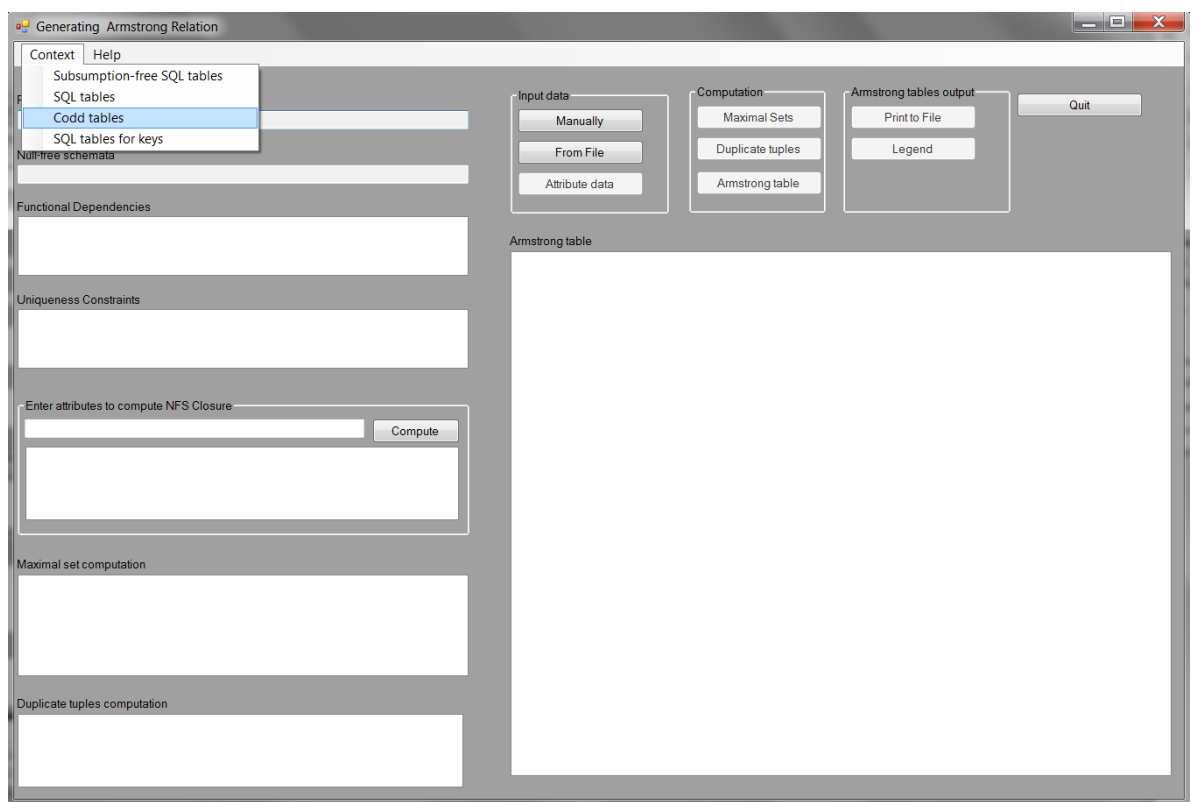

Figure 5.9: Screenshot of Selecting the Context in the Use Case Example

\subsubsection{Computing Armstrong Table}

Figure 5.11 contains a screenshot of the Armstrong table computed by SQL-Sampler on the basis of the input data. Since no domain values had been supplied by the user, the Armstrong table was populated with generic data values. The screenshot also shows the maximal and duplicate sets computed by SQL-Sampler.

\subsubsection{Output}

Figure 5.12 shows a screenshot of an Armstrong table for the input data after the generic domain values have been replaced by real data values. This was done after the "Legend" button in the "Armstrong table output" menu was activated by the user, and the generic data values were manually replaced by the real data values. 


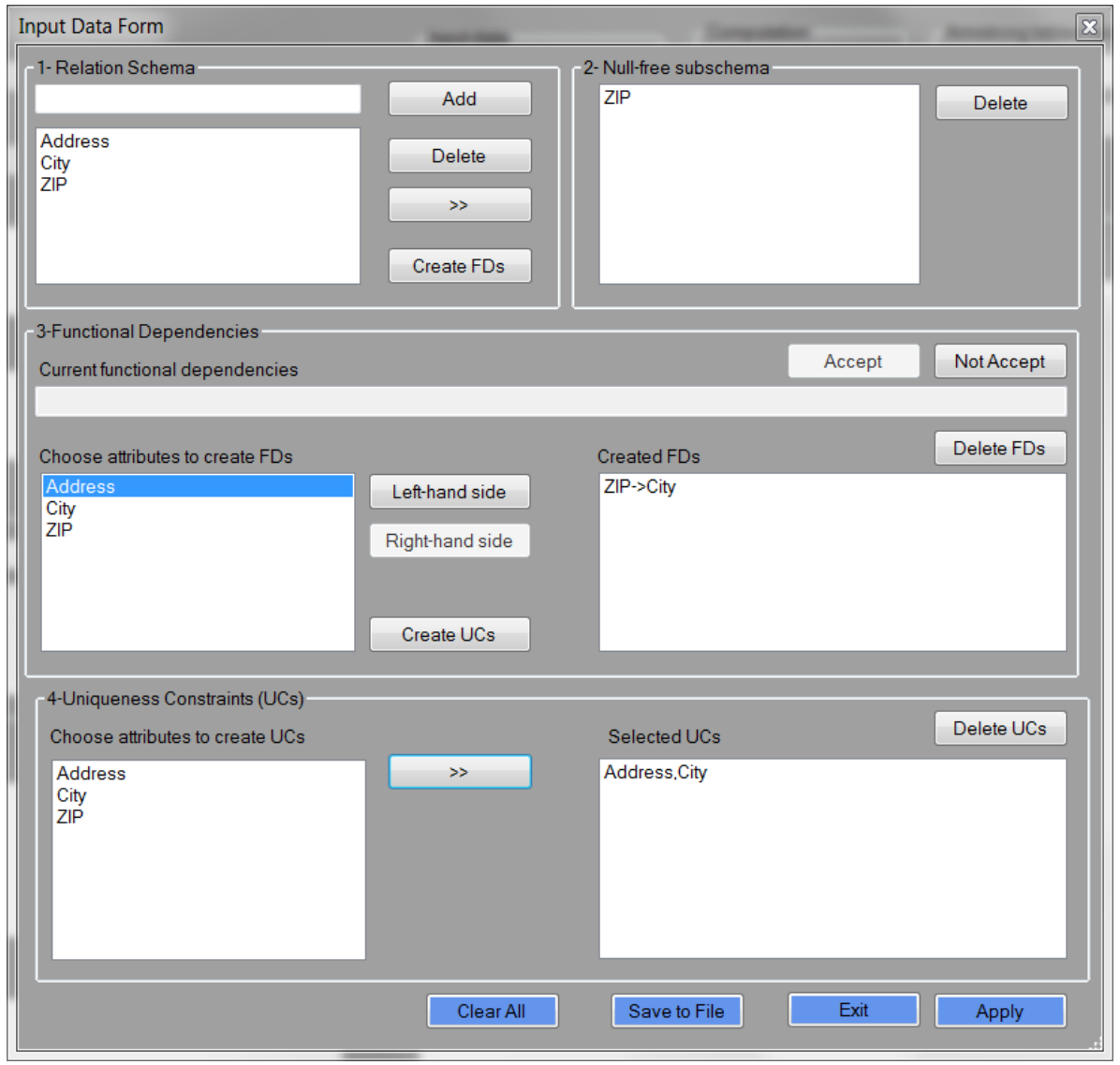

Figure 5.10: Screenshot of Putting in Data in the Use Case Example 


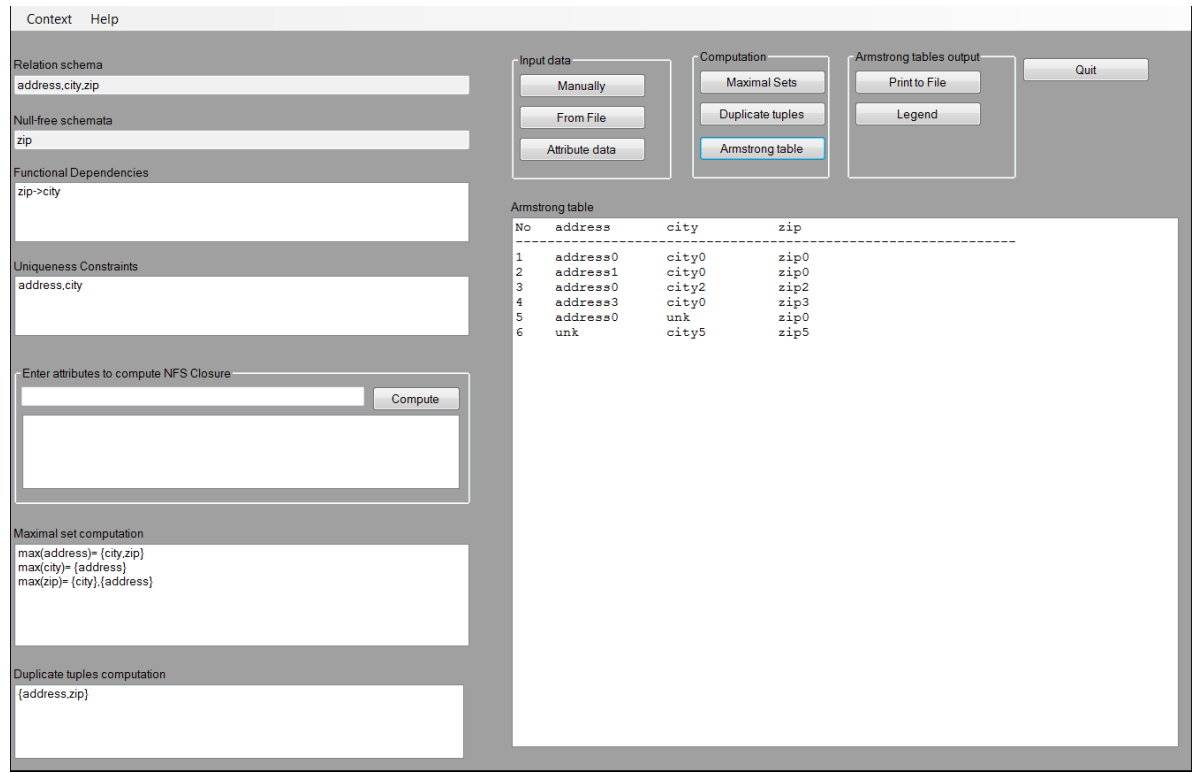

Figure 5.11: Screenshot of Armstrong Table in the Use Case Example

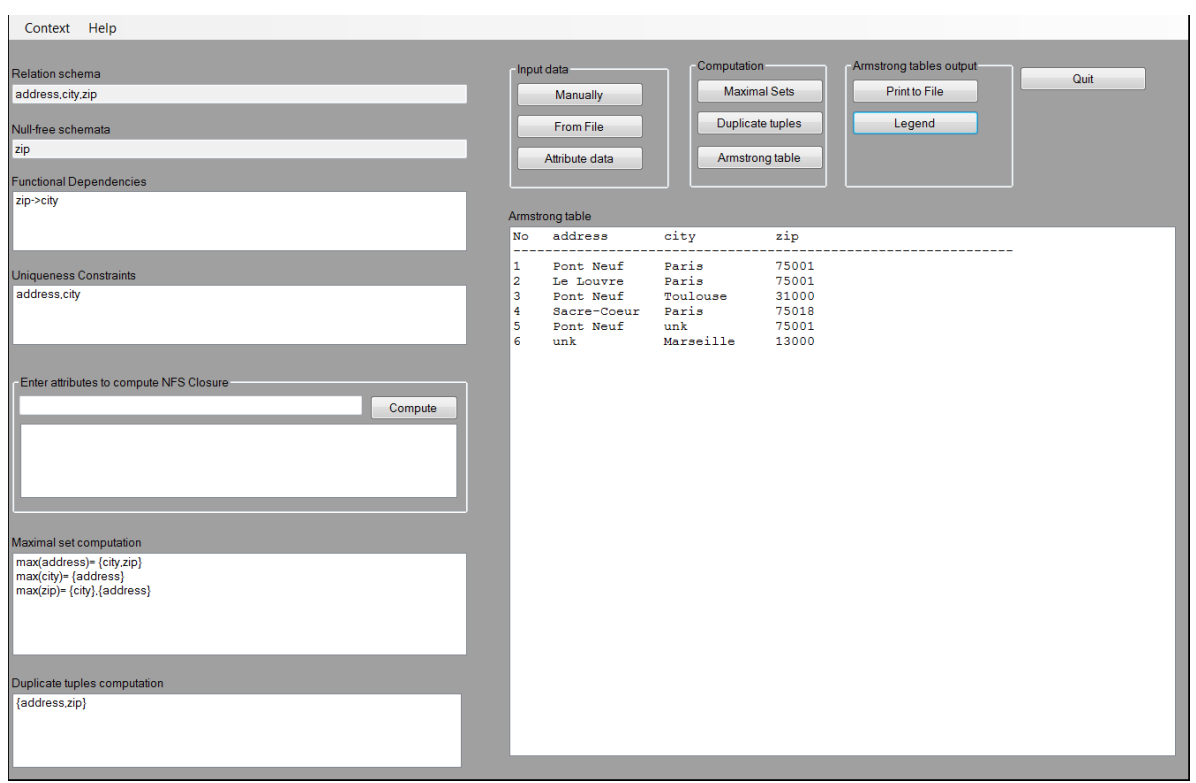

Figure 5.12: Screenshot of Output Data in the Use Case Example 


\subsection{Summary}

This chapter described how the second objective of this thesis has been addressed. In fact, the implementation of a toolbox, called SQL-Sampler, was detailed that computes Armstrong tables with respect to different classes of constraints and different interpretations of null markers. The graphical user interface and functionality of SQL-Sampler was described for its desktop version and its Web-based version. The design was divided into four modules that are driven by a back-end database in case of the Webbased version. The functions of each module were explained. Finally, a use case scenario was illustrated by a series of screen shots. The functionality of SQL-Sampler forms the basis for the empirical evaluation of the usefulness of Armstrong tables for the acquisition of semantically meaningful constraints, as detailed in the next couple of chapters. 


\section{Chapter 6}

\section{Evaluation - Part I: Experiment Design}

In this and the next chapter the third and final goal of this thesis is addressed, as stated at the end of chapter 2. The goal is to investigate empirically whether Armstrong tables, as produced by SQL-Sampler, really are useful for the acquisition of semantically meaningful constraints. The goal means that measures will first need to be defined that provide us with a precise definition of the term useful. Subsequently, experiments with design teams can be conducted that apply the measures to observe the extent by which an inspection of Armstrong tables is indeed useful for the identification of semantically meaningful constraints. The focus of this chapter is on the combined class of uniqueness constraints and functional dependencies with nulls over SQL tables with a null-free subschema.

The measures introduced in this chapter were announced in the conference paper [81].

This chapter is organized as follows: we describe the overall process of the experiment in Section 6.1 and the characteristics of participants in the experiment in Section 6.2. We introduce the application domain and the target set of constraints used in the experiment in Section 6.3. In Section

6.4 we introduce three different measures, namely soundness, completeness, 
and proximity, to evaluate the quality of the constraints. Section 6.5 illustrates the measures on a running example taken from the data that was collected during the experiments. Finally, in Section 6.6, we discuss limitations of our experiments. The following chapter includes the quantitative and qualitative analysis of the data from our experiments.

\subsection{The overall process of the experiment}

It has long been speculated that the inspection of Armstrong tables helps database designers with the discovery of semantically meaningful constraints [77]. We are describing an experiment that is suitable to gather empirical evidence for this hypothesis with respect to the SQL constraints under investigation.

The experiment utilizes the pre-test post-test model [32, 85] to measure the extent by which the inspection of Armstrong tables improves the discovery of SQL constraints. In the pre-test, participants indicate which SQL constraints they perceive as semantically meaningful without having available any sample data. In the post-test, participants revise their perceptions based on Armstrong tables produced by SQL-Sampler for the set of SQL constraints they currently perceive meaningful. Intuitively, Armstrong tables are considered to be useful if the quality of the constraint sets which are perceived as meaningful by the participants improves from the pre-test to the post-test phase. Several measures are proposed to evaluate the quality of the constraint sets qualitatively and quantitatively.

Figure 6.1 illustrates the pre-test and post-test phases of the experiment. In the first phase, the pre-test phase, the participants are asked to write down a cover for the set of NUCs and NFDs that they perceive as semantically meaningful for a given application domain. They are also encouraged to ask questions regarding the application domain to the person acting as domain experts. The person acting as domain expert is only allowed to answer questions from the participants in plain English. At 
Figure 6.1: Overview of the experiment

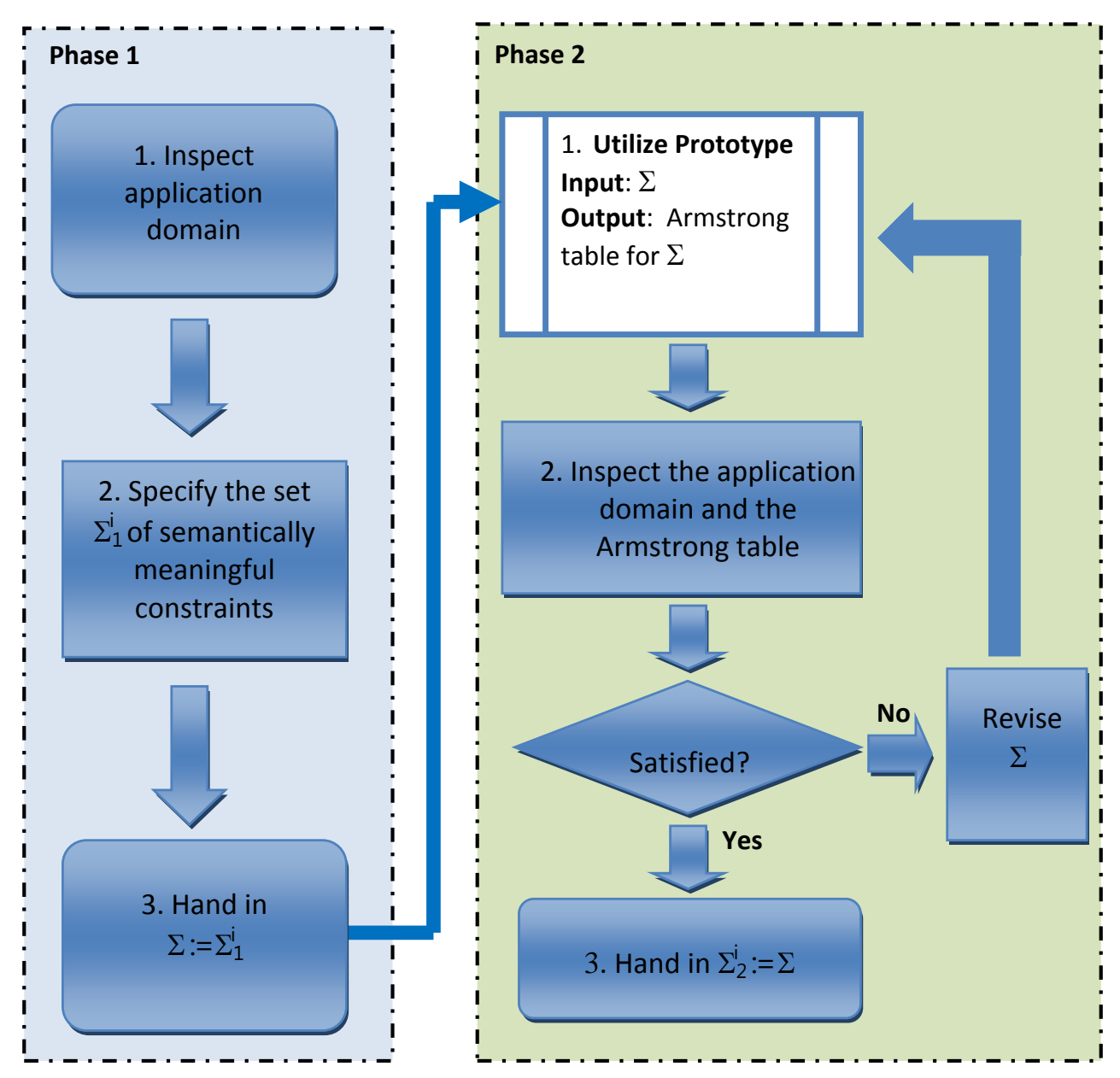

the end of the first phase each participating design team $i$ hands in their constraint set $\Sigma_{1}^{i}$.

At the beginning of the second phase, the post-test phase, each design team $i$ is provided with an Armstrong table for their corresponding con- 
straint set $\Sigma_{1}^{i}$. Each design team then investigates the provided sample table and, if necessary, revises their constraint set. In particular, they can ask the domain experts further questions with regard to the application domain. The questions may include direct references to the Armstrong table. During the second phase, each design team can produce several revised versions of its constraint set and can access the corresponding Armstrong tables. This process continues until the design team is satisfied with the result. Eventually, each team hands in a final constraint set $\Sigma_{2}^{i}$.

\subsection{Participants}

The experiment was conducted in three different universities: The University of Auckland (AU), Victoria University of Wellington (VUW), and Lotus University of Vietnam (LU). A total of 50 teams participated in the experiments. Each team consisted of two to three students. Each team was assigned a unique number $i$ between 1 and 50 .

The students that took part in the experiment were familiar with fundamental database concepts such as uniqueness constraints, functional dependencies, and implied constraints. The students were not taught the formal concept of Armstrong tables before the experiment. They were informed that the tables they are given constitute perfect representations of the constraint set $\Sigma$ they have just specified, in the sense that the table satisfies an NUC or NFD if it is implied by $\Sigma$, and violated otherwise.

Data from AU was collected from fourth-year post-graduate computer science students divided into 12 teams. The students from VUW were from the School of Engineering and Computer Science (CS) or from the School of Information Management (IS). They were undergraduate students taking a third-year database course. They formed 22 teams. The students from LU were enrolled in a third-year undergraduate database course offered by the School of Computer Science. The mix of students with different background added to the robustness of the experiment. 
The author of this research and her supervisors acted as domain experts for the application domain, as described in detail below. There was a concerted attempt to mimic a real-world situation as closely as possible. For example, the domain experts answered questions by the design teams regarding the application domain, but the design teams only received answers to non-technical questions in plain English. Questions of the kind "Does this functional dependency make sense?" were not answered, as domain experts cannot be expected to be familiar with the database concept of a functional dependency.

\subsection{Application domain and the target set}

A WORK relation schema with five attributes Proj, Emp, Date, Role, Hrs was used in the experiment. The schema contains information related to projects (using attribute Proj), employees (identified by attribute Emp), date, roles, and hours (using attribute by Hrs). It records the number of hours (e.g., 5) that an employee (e.g., Dilbert) works on a project (e.g., Blue) in some role (e.g., Programmer) on some day (e.g., Oct 5). The application domain defines $\mathrm{WORK}_{s}=\{$ Date,Emp $\}$ as null-free subschema, which means that the null marker $n i$ cannot occur in the Emp and Date columns.

The following NUCs and NFDs over WORK constitute the target set $\Sigma^{t}$ which is a cover of the semantically meaningful NUCs and NFDs over WORK:

- $u($ Date, Emp)

- Emp,Proj $\rightarrow$ Role, Hrs

- Proj,Role $\rightarrow$ Hrs

Note that the NFD Emp,Proj $\rightarrow$ Hrs is not implied by the NUC and the NFDs Emp,Proj $\rightarrow$ Role and Proj,Role $\rightarrow$ Hrs, under the given null-free subschema. 


\subsection{Quality measures}

This section introduces several quality measures that allow us to evaluate the quality of a set $\Sigma$ of NUCs and NFDs with respect to the target set $\Sigma^{t}$ and the NFS $n f_{S}\left(R_{s}\right)$ over relation schema $R$. We give some preliminary notation.

\subsubsection{Preliminary notation}

For a set $\Sigma$ of NUCs and NFDs, and an NFS $n f_{s}\left(R_{s}\right)$ over relation schema $R$ we define $s(\Sigma)$ as the set of NUCs implied by $\Sigma$ :

$$
s(\Sigma)=\left\{u(X) \mid \emptyset \neq X \subseteq R \wedge \Sigma \models_{R_{s}} u(X)\right\} .
$$

Take the target set $\Sigma^{t}$ from our application domain as an example. Then we have:

$$
\begin{aligned}
s\left(\Sigma^{t}\right):= & \{u(\text { Date,Emp }), u(\text { Date,Emp,Hrs }), u(\text { Date,Emp,Hrs,Proj }), \\
& u(\text { Date,Emp,Hrs,Role }), u(\text { Date,Emp,Proj }), u(\text { Date,Emp,Role }), \\
& u(\text { Date,Emp,Proj,Role }), u(\text { Date,Emp,Hrs,Proj,Role })\} .
\end{aligned}
$$

Recall that the attribute set closure $X_{\Sigma, R_{s}}^{*}$ for an attribute set $X \subseteq R$ and NFS $n f s\left(R_{s}\right)$ is defined as follows:

$$
X_{\Sigma, R_{s}}^{*}=\left\{A \in R \mid \Sigma \models_{R_{s}} X \rightarrow A\right\} .
$$

Table 6.1 shows the attribute set closure for each non-empty attribute set over WORK with respect to our target set $\Sigma^{t}$ and the NFS $n f_{s}\left(R_{s}\right)$. The table also indicates for each non-empty attribute set $X$ whether the NUC $u(X)$ is an element of $s\left(\Sigma^{t}\right)$.

As both uniqueness constraints and functional dependencies are considered the measures of soundness, completeness, and proximity are separated into measures for i) uniqueness constraints and ii) functional dependencies. Both measures are determined by both uniqueness constraints 


\begin{tabular}{|c|c|c|}
\hline$X \subseteq$ WORK & $X_{\Sigma^{t}, R_{s}}^{*}$ & $\Sigma \models R_{R_{s}} u(X) ?$ \\
\hline Date & Date & no \\
\hline Date,Emp & Date,Emp,Hrs,Proj,Role & yes \\
\hline Date,Emp,Hrs & Date,Emp,Hrs,Proj,Role & yes \\
\hline Date,Emp,Hrs,Proj & Date,Emp,Hrs,Proj,Role & yes \\
\hline Date,Emp,Hrs,Proj,Role & Date,Emp,Proj,Role,Hrs & yes \\
\hline Date,Emp,Hrs,Role & Date,Emp,Hrs,Role,Proj & yes \\
\hline Date,Emp,Proj & Date,Emp,Proj,Hrs,Role & yes \\
\hline Date,Emp,Proj,Role & Date,Emp,Proj,Role,Hrs & yes \\
\hline Date,Emp,Role & Date,Emp,Role,Hrs,Proj & yes \\
\hline Date,Hrs & Date,Hrs & no \\
\hline Date,Hrs,Proj & Date,Hrs,Proj & no \\
\hline Date,Hrs,Proj,Role & Date,Hrs,Proj,Role & no \\
\hline Date,Hrs,Role & Date,Hrs, Role & no \\
\hline Date,Proj & Date,Proj & no \\
\hline Date,Proj,Role & Date,Proj,Role,Hrs & no \\
\hline Date,Role & Date,Role & no \\
\hline Emp & Emp & no \\
\hline Emp,Hrs & Emp,Hrs & no \\
\hline Emp,Hrs,Proj & Emp,Hrs,Proj,Role & no \\
\hline Emp,Hrs,Proj,Role & Emp,Hrs,Proj,Role & no \\
\hline Emp,Hrs,Role & Emp,Hrs,Role & no \\
\hline Emp,Proj & Emp,Hrs,Proj,Role & no \\
\hline Emp,Proj,Role & Emp,Hrs,Proj,Role & no \\
\hline Emp,Role & Emp,Role & no \\
\hline Hrs & Hrs & no \\
\hline Hrs,Proj & Hrs,Proj & no \\
\hline Hrs,Proj,Role & Hrs,Proj,Role & no \\
\hline Hrs,Role & Hrs,Role & no \\
\hline Proj & Proj & no \\
\hline Proj,Role & Hrs,Proj,Role & no \\
\hline Role & Role & no \\
\hline
\end{tabular}

Table 6.1: Closures and implied uniqueness constraints under the target set $\Sigma^{t}$ and the NFS $n f_{S}\left(\right.$ WORK $\left._{s}\right)$ 
and functional dependencies, because of their interaction. This interaction is completely captured by the axiomatization in Theorem 3.1. In particular, this means uniqueness constraints imply functional dependencies, and functional dependencies together with some uniqueness constraints may imply other uniqueness constraints. Non-standard uniqueness constraints and functional dependencies are not considered since they do not enjoy Armstrong tables [64].

\subsubsection{Soundness}

Soundness measures which of the constraints perceived meaningful by a design team are actually meaningful. Here, actually meaningful are the constraints implied by the target set $\Sigma^{t}$. The soundness for uniqueness constraints of a set $\Sigma$ with respect to a target set $\Sigma^{t}$ is defined as the ratio between the as meaningful perceived uniqueness constraints implied by $\Sigma^{t}$ and all the as meaningful perceived uniqueness constraints, that is:

$$
\operatorname{sound}_{\Sigma^{t}}^{u}(\Sigma)=\left\{\begin{array}{ll}
1 & \text { if } s(\Sigma)=\emptyset \\
\frac{\left|s(\Sigma) \cap s\left(\Sigma^{t}\right)\right|}{|s(\Sigma)|}, & \text { otherwise }
\end{array} .\right.
$$

Note that, if $\operatorname{sound}_{\Sigma^{t}}^{u}(\Sigma)=1$, then every uniqueness constraint that is perceived as meaningful is actually meaningful. By contrast, if $\operatorname{sound} d_{\Sigma^{t}}^{u}=$ 0 , then none of the uniqueness constraints perceived as meaningful are actually meaningful.

Recall that $\mathcal{P}_{0}(R)=\{X \subseteq R \mid X \neq \emptyset \wedge X \neq R\}$ denotes the set of nonempty attribute subsets of $R$. The soundness for functional dependencies of a set $\Sigma$ with respect to the target set $\Sigma^{t}$ is the ratio between the attribute subsets in $\mathcal{P}_{0}(R)$ whose closure under $\Sigma$ is contained in the closure under $\Sigma^{t}$, and $\mathcal{P}_{0}(R)$, that is:

$$
\operatorname{sound}_{\Sigma^{t}}^{f}(\Sigma)=\frac{\left|\left\{X \in \mathcal{P}_{0}(R) \mid X_{\Sigma}^{*} \subseteq X_{\Sigma^{t}}^{*}\right\}\right|}{\left|\mathcal{P}_{0}(R)\right|} .
$$

If $\operatorname{sound}_{\Sigma^{t}}^{f}(\Sigma)=1$, then all the functional dependencies perceived as meaningful are actually meaningful. By contrast, if $\operatorname{sound}_{\Sigma^{t}}^{f}=0$, then none 
of the functional dependencies perceived as meaningful is actually meaningful.

\subsubsection{Completeness}

Completeness measures which of the actually meaningful constraints are also perceived as meaningful by a team. Completeness for uniqueness constraints is thus the ratio between the actually meaningful uniqueness constraints that are also perceived as meaningful, and the actually meaningful uniqueness constraints, that is:

$$
\text { complete }_{\Sigma^{t}}^{u}(\Sigma)=\left\{\begin{array}{ll}
1 & , \text { if } s\left(\Sigma^{t}\right)=\emptyset \\
\frac{\left|s(\Sigma) \cap s\left(\Sigma^{t}\right)\right|}{\left|s\left(\Sigma^{t}\right)\right|} & , \text { otherwise }
\end{array} .\right.
$$

If complete $\Sigma_{\Sigma^{t}}^{u}(\Sigma)=1$, then every actually meaningful uniqueness constraint is also perceived as meaningful. By contrast, if $\operatorname{complete}_{\Sigma^{t}}^{u}(\Sigma)=0$, then none of the actually meaningful uniqueness constraints is perceived as meaningful.

Completeness for functional dependencies is the ratio between the attribute sets in $\mathcal{P}_{0}(R)$ whose closure under $\Sigma^{t}$ is contained in the closure under $\Sigma$, and $\mathcal{P}_{0}(R)$, that is:

$$
\operatorname{complete}_{\Sigma^{t}}^{f}(\Sigma)=\frac{\left|\left\{X \in \mathcal{P}_{0}(R) \mid X_{\Sigma^{t}}^{*} \subseteq X_{\Sigma}^{*}\right\}\right|}{\left|\mathcal{P}_{0}(R)\right|} .
$$

If complete $\Sigma_{\Sigma^{t}}^{f}(\Sigma)=1$, then every actually meaningful functional dependency is also perceived as meaningful. By contrast, if complete $\Sigma_{\Sigma^{t}}^{f}(\Sigma)=0$, then none of the actually meaningful functional dependencies is perceived as meaningful.

\subsubsection{Proximity}

Proximity measures how close two sets of constraints are. For uniqueness constraints it is the ratio between the as meaningful perceived uniqueness 
constraints that are actually meaningful, and all the actually meaningful and all the as meaningful perceived uniqueness constraints, that is:

$$
\operatorname{prox}^{u}\left(\Sigma, \Sigma^{t}\right)=\left\{\begin{array}{ll}
1 & , \text { if } s(\Sigma) \cup s\left(\Sigma^{t}\right)=\emptyset \\
\frac{\left|s(\Sigma) \cap s\left(\Sigma^{t}\right)\right|}{\left|s(\Sigma) \cup s\left(\Sigma^{t}\right)\right|}, & \text { otherwise }
\end{array} .\right.
$$

Note that if $\operatorname{prox}_{\Sigma^{t}}^{u}(\Sigma)=1$, there is no distance between the actually meaningful uniqueness constraints and those perceived as meaningful. In contrast, if $\operatorname{prox}_{\Sigma^{t}}^{u}(\Sigma)=0$, then uniqueness constraints perceived as meaningful and uniqueness constraints that are actually meaningful form disjoint sets.

For

$$
\operatorname{dist}^{u}\left(\Sigma, \Sigma^{t}\right)=\left(s(\Sigma)-s\left(\Sigma^{t}\right)\right) \cup\left(s\left(\Sigma^{t}\right)-s(\Sigma)\right),
$$

$\left|\operatorname{dist}^{u}\left(\Sigma, \Sigma^{t}\right)\right|$ defines a metric on equivalent sets of uniqueness constraints. Indeed, dist $t^{u}\left(\Sigma, \Sigma^{t}\right)$ is the symmetric difference of $s(\Sigma)$ and $s\left(\Sigma^{t}\right)$.

For functional dependencies, completeness is the ratio between the attribute sets in $\mathcal{P}_{0}(R)$ whose closure under $\Sigma^{t}$ is the same as the closure under $\Sigma$, and $\mathcal{P}_{0}(R)$ :

$$
\operatorname{prox}^{f}\left(\Sigma, \Sigma_{t}\right)=\frac{\left|\left\{X \in \mathcal{P}_{0}(R) \mid X_{\Sigma_{t}}^{*}=X_{\Sigma}^{*}\right\}\right|}{\left|\mathcal{P}_{0}(R)\right|} .
$$

Similar to uniqueness constraints, for

$$
\operatorname{dist}^{f}\left(\Sigma, \Sigma^{t}\right)=\left\{X \in \mathcal{P}_{0}(R) \mid X_{\Sigma^{t}}^{*} \neq X_{\Sigma}^{*}\right\},
$$

$\left|\operatorname{dist}^{f}\left(\Sigma, \Sigma^{t}\right)\right|$ defines a metric on equivalent sets of functional dependencies.

\subsubsection{Gain}

For each quality measure that was just introduced, we calculate for each team how much they gained in terms of this measure by inspecting Armstrong tables, that is, by going from Phase 1 to Phase 2. Gain is then defined as the arithmetic mean. 
Recall that $i=1, \ldots, 50$ identifies each of our 50 design teams, $\Sigma_{1}^{i}$ and $\Sigma_{2}^{i}$ denote the sets of uniqueness constraints and functional dependencies perceived semantically meaningful by design team $i$ after Phase 1 and 2, respectively, and $\Sigma^{t}$ denotes the target set of uniqueness constraints and functional dependencies. For $a=u$ or $a=f$, let

- gain-in-sound ${ }_{i}^{a}=\operatorname{sound}_{\Sigma^{t}}^{a}\left(\sum_{2}^{i}\right)-\operatorname{sound}_{\Sigma^{t}}^{a}\left(\Sigma_{1}^{i}\right)$,

- gain-in-complete $_{i}^{a}=\operatorname{complete}_{\Sigma^{t}}^{a}\left(\Sigma_{2}^{i}\right)-\operatorname{complete}_{\Sigma^{t}}^{a}\left(\Sigma_{1}^{i}\right)$,

- gain-in-prox $i_{i}^{a}=\operatorname{prox}^{a}\left(\Sigma^{t}, \Sigma_{2}^{i}\right)-\operatorname{prox}^{a}\left(\Sigma^{t}, \Sigma_{1}^{i}\right)$.

The gain for every measure in sound, complete and prox, respectively, from Phase 1 to Phase 2 of the experiment, is defined as the arithmetic mean over the measures taken for each team:

$$
\text { gain-in-measure }^{a}=\frac{\sum_{i=1}^{n} \text { gain-in-measure }_{i}^{a}}{n}
$$

where $n$ is the total number of teams involved in the experiment, that is, 50 in our case.

For every measure in $\{$ sound,complete,prox $\}$ we say that the use of Armstrong tables for the discovery of uniqueness constraints, functional dependencies respectively, achieved a gain of gain-in-measure ${ }^{a} \times 100 \%$. We consider that Armstrong tables are useful for the discovery of uniqueness constraints, functional dependencies respectively, in terms of these measures if their respective values for the gain gain-in-measure ${ }^{a}$ is positive.

\subsubsection{Actual and possible gain}

In this section, some relativized versions of the measures for gains are introduced. These illustrate the usefulness better than the previous measures as they show what has actually been gained relative to what could have possibly been gained. 
Intuitively speaking, the possible gain of a measure is the maximal gain possible to achieve for a team in Phase 2 of the experiment. More formally, one can define for $a \in\{u, f\}$ :

- possible-gain-in-sound $^{a}=100 \% \cdot\left(1-\operatorname{sound}_{\Sigma^{t}}^{a}\left(\Sigma_{1}^{i}\right)\right)$,

- possible-gain-in-complete ${ }^{a}=100 \% \cdot\left(1-\operatorname{complete}_{\Sigma^{t}}^{a}\left(\Sigma_{1}^{i}\right)\right)$, and

- possible-gain-in-prox ${ }^{a}=100 \% \cdot\left(1-\operatorname{prox}^{a}\left(\Sigma_{1}^{i}, \Sigma^{t}\right)\right)$

Possible gains should be contrasted with actual gains. For every measure in sound, complete, prox and $a \in\{u, f\}$ we have:

actual-gain-in-measure ${ }_{i}^{a}=$ gain-in-measure $_{i}^{a}$.

\subsection{Example}

The measures illustrated in the previous section are now illustrated on a running example taken from design team 2 in the actual experiments.

\subsubsection{Data gathering}

At the beginning of the experiment all teams were provided with an Information Sheet describing the steps of the experiment and a worksheet describing the application domain. Recall that the application domain relates to the relation schema WORK $=\{$ Emp, Date, Proj, Role, Hrs $\}$ and an NFS $W_{\text {ORK }}=\{$ Date, Emp $\}$. Teams were allowed to check their assumptions regarding the application domain with the domain experts present. After 30 minutes each team was asked to hand in the set of uniqueness constraints and functional dependencies that they perceived as meaningful for the described application domain. For instance, Team 2 handed in the following set.

$$
\Sigma_{1}^{2}=\{u(\text { Date,Emp,Proj }) ; \text { Proj,Role } \rightarrow \text { Hrs }\}
$$


We then produced an Armstrong table for each set $\Sigma_{1}^{i}$ of constraints handed in by the teams. In the next phase of the experiment, each team $i$ was given their corresponding Armstrong table for $\Sigma_{1}^{i}$, and allowed to revise their constraint set. Table 6.2 shows the actual Armstrong table returned to Team 2. A new Armstrong table was produced for each new set of constraints handed in by a team. This refinement process continued until teams were happy with their result or the time of 1 hour, allowed for the task, expired. The following set of constraints is the final set produced by Team 2 .

$$
\Sigma_{2}^{2}=\{u(\text { Date,Emp }) ; \text { Proj,Role } \rightarrow \text { Hrs; Emp,Proj } \rightarrow \text { Role }\}
$$

\subsubsection{Data analysis}

In what follows we describe how the measures that have been introduced in this chapter apply to the data gathered from team 2.

The quality of constraint sets obtained from each team $i$ in Phase 1 and 2, respectively, is measured on the basis of attribute set closures and implied uniqueness constraints. For team 2, for example, Table 6.3 and Table 6.4 show the closures and implied uniqueness constraints for Phase 1 and Phase 2 , respectively.

\section{Phase 1}

We show next the soundness, completeness, and proximity measures corresponding to the constraints specified by Team 2 in Phase 1.

Team 2 achieved the following soundness for uniqueness constraints in the first phase of the experiment:

$$
\operatorname{sound}_{\Sigma^{t}}^{u}\left(\Sigma_{1}^{2}\right)=\frac{4}{4}=1
$$




\begin{tabular}{lllll}
\hline Date & Emp & Hrs & Proj & Role \\
\hline 01 Jan & Mickey & 2.5 & Red & Analyst \\
02 Jan & Mickey & 2.5 & Red & Analyst \\
03 Jan & Donald & 3 & Blue & Consultant \\
03 Jan & Pluto & 3 & Blue & Consultant \\
04 Jan & Goofy & 4 & $\mathrm{ni}$ & Programmer \\
04 Jan & Goofy & 5.5 & $\mathrm{ni}$ & Programmer \\
05 Feb & Dale & 6 & Black & ni \\
12 Feb & Dale & 7 & Black & ni \\
15 Mar & Batman & 8 & White & ni \\
15 Mar & Dilbert & 9 & White & ni \\
16 Apr & Spiderman & 4.5 & Organge & Security \\
16 Apr & Spiderman & 4.5 & Magenta & Security \\
17 May & Catwoman & 5 & Purple & Planner \\
18 Jun & Catwoman & 5 & Purple & Doctor \\
20 Jun & Storm & 2 & Silver & Manager \\
20 Jun & Hulk & 2 & Silver & Developer \\
21 Jul & Tinkerbell & 6.5 & ni & Mathematician \\
$21 \mathrm{Jul}$ & Tinkerbell & 6.5 & ni & Statistician \\
22 Jul & Oliver & $n i$ & Gold & Physicist \\
\hline
\end{tabular}

Table 6.2: Armstrong table for the constraint set $\{u$ (Date,Emp,Proj); Proj,Role $\rightarrow$ Hrs $\}$ over WORK and NFS WORK W $_{s}=\{$ Date, Emp $\}$

Indeed, all of the four uniqueness constraints implied by $\Sigma_{1}^{2}$ are actually meaningful. The only other functional dependency Proj,Role $\rightarrow$ Hrs, perceived meaningful by team 2 , is indeed meaningful. Therefore,

$$
\operatorname{sound}_{\Sigma^{t}}^{f}\left(\Sigma_{1}^{2}\right)=1 \text {. }
$$

Regarding the completeness of uniqueness constraints, out of the 8 actually meaningful uniqueness constraints, only 4 are implied by $\Sigma_{1}^{2}$, that 


\begin{tabular}{|c|c|c|}
\hline$X \subseteq$ WORK & Closure $X_{\Sigma_{1}^{2}, \text { WORK }_{s}}^{*}$ & $\Sigma_{1}^{2} \models$ WORK $_{s} u(X) ?$ \\
\hline Date & Date & no \\
\hline Date,Emp & Date,Emp & no \\
\hline Date,Emp,Hrs & Date,Emp,Hrs & no \\
\hline Date,Emp,Hrs,Proj & Date,Emp,Hrs,Proj,Role & yes \\
\hline Date,Emp,Hrs,Proj,Role & Date,Emp,Hrs,Proj,Role & yes \\
\hline Date,Emp,Hrs,Role & Date,Emp,Hrs,Role & no \\
\hline Date,Emp,Proj & Date,Emp,Proj,Hrs,Role & yes \\
\hline Date,Emp,Proj,Role & Date,Emp,Proj,Role,Hrs & yes \\
\hline Date,Emp,Role & Date,Emp,Role & no \\
\hline Date,Hrs & Date,Hrs & no \\
\hline Date,Hrs,Proj & Date,Hrs,Proj & no \\
\hline Date,Hrs,Proj,Role & Date,Hrs,Proj,Role & no \\
\hline Date,Hrs,Role & Date,Hrs,Role & no \\
\hline Date,Proj & Date,Proj & no \\
\hline Date,Proj,Role & Date,Proj,Role,Hrs & no \\
\hline Date,Role & Date,Role & no \\
\hline Emp & Emp & no \\
\hline Emp,Hrs & Emp,Hrs & no \\
\hline Emp,Hrs,Proj & Emp,Hrs,Proj & no \\
\hline Emp,Hrs,Proj,Role & Emp,Hrs,Proj,Role & no \\
\hline Emp,Hrs,Role & Emp,Hrs,Role & no \\
\hline Emp,Proj & Emp,Proj & no \\
\hline Emp,Proj,Role & Emp,Proj,Role,Hrs & no \\
\hline Emp,Role & Emp,Role & no \\
\hline Hrs & Hrs & no \\
\hline Hrs,Proj & Hrs,Proj & no \\
\hline Hrs,Proj,Role & Hrs,Proj,Role & no \\
\hline Hrs, Role & Hrs,Role & no \\
\hline Proj & Proj & no \\
\hline Proj,Role & Proj,Role,Hrs & no \\
\hline Role & Role & no \\
\hline
\end{tabular}

Table 6.3: Closures and implied uniqueness constraints for $\Sigma_{1}^{2}$ 


\begin{tabular}{|c|c|c|}
\hline$X \subseteq$ WORK & Closures $X_{\Sigma_{2}^{2}, \text { WORK }_{s}}^{*}$ & $\Sigma_{2}^{2}=_{R_{s}} u(X) ?$ \\
\hline Date & Date & no \\
\hline Date,Emp & Date,Emp,Hrs,Proj,Role & yes \\
\hline Date,Emp,Hrs & Date,Emp,Hrs,Proj,Role & yes \\
\hline Date,Emp,Hrs,Proj & Date,Emp,Hrs,Proj,Role & yes \\
\hline Date,Emp,Hrs,Role & Date,Emp,Hrs,Role,Proj & yes \\
\hline Date,Emp,Proj & Date,Emp,Proj,Role,Hrs & yes \\
\hline Date,Emp,Proj,Role & Date,Emp,Proj,Role,Hrs & yes \\
\hline Date,Emp,Proj,Role,Hrs & Date,Emp,Proj,Role,Hrs & yes \\
\hline Date,Emp,Role & Date,Emp,Role,Hrs,Proj & yes \\
\hline Date,Hrs & Date,Hrs & no \\
\hline Date,Hrs,Proj & Date,Hrs,Proj & no \\
\hline Date,Hrs,Proj,Role & Date,Hrs,Proj,Role & no \\
\hline Date,Hrs,Role & Date,Hrs,Role & no \\
\hline Date,Proj & Date,Proj & no \\
\hline Date,Proj,Role & Date,Proj,Role,Hrs & no \\
\hline Date,Role & Date,Role & no \\
\hline Emp & Emp & no \\
\hline Emp,Hrs & Emp,Hrs & no \\
\hline Emp,Hrs,Proj & Emp,Hrs,Proj,Role & no \\
\hline Emp,Hrs,Proj,Role & Emp,Hrs,Proj,Role & no \\
\hline Emp,Hrs,Role & Emp,Hrs,Role & no \\
\hline Emp,Proj & Emp,Proj,Role & no \\
\hline Emp,Proj,Role & Emp,Proj,Role,Hrs & no \\
\hline Emp,Role & Emp,Role & no \\
\hline Hrs & $H r s$ & no \\
\hline Hrs,Proj & Hrs,Proj & no \\
\hline Hrs,Proj,Role & Hrs,Proj,Role & no \\
\hline Hrs, Role & Hrs, Role & no \\
\hline Proj & Proj & no \\
\hline Proj,Role & Proj,Role,Hrs & no \\
\hline Role & Role & no \\
\hline
\end{tabular}

Table 6.4: Closures and implied uniqueness constraint for $\Sigma_{2}^{2}$ 
is:

$$
\operatorname{complete}_{\Sigma^{t}}^{u}\left(\Sigma_{1}^{2}\right)=\frac{4}{8}=0.5 .
$$

Several actually meaningful functional dependencies were not perceived meaningful by team 2 in Phase 1, in fact:

$$
\operatorname{complete}_{\Sigma^{t}}^{f}\left(\Sigma_{1}^{2}\right)=\frac{25}{31} \simeq 0.81
$$

Indeed, the six violating sets are:

$$
\begin{aligned}
\left\{X \in \mathcal{P}_{0}(R) \mid X_{\Sigma^{t}}^{*} \subseteq X_{\Sigma}^{*}\right\}= & \mathcal{P}_{0}(R)-\{\{\text { Date,Emp }\},\{\text { Date,Emp,Hrs }\} \\
& \{\text { Date,Emp,Hrs,Role }\},\{\text { Date,Emp,Role }\} \\
& \{\text { Emp,Hrs,Proj }\},\{\text { Emp,Proj }\}\}
\end{aligned}
$$

Since the soundness for uniqueness constraints and functional dependencies is both 1 , completeness and proximity coincide, that is:

- $\operatorname{prox}^{u}\left(\Sigma_{1}^{2}, \Sigma^{t}\right)=\frac{4}{8}=0.5$,

- $\operatorname{prox}^{f}\left(\Sigma_{1}^{2}, \Sigma^{t}\right)=\frac{25}{31} \simeq 0.81$.

In conclusion, in phase 1 team 2 handed in a fully sound set $\Sigma_{1}^{2}$ of constraints that is half complete in terms of uniqueness constraints and about 80 percent in terms of functional dependencies.

\section{Phase 2}

After Phase 2, team 2 had replaced their NUC $u$ (Date, Emp, Proj) with the uniqueness constraint $u$ (Date, Emp), and added the functional dependency Emp, Proj $\rightarrow$ Role. Both the new uniqueness constraint and the new functional dependency are actually meaningful and, therefore, the soundness for uniqueness constraints calculates as

$$
\operatorname{sound}_{\Sigma^{t}}^{u}\left(\Sigma_{2}^{2}\right)=\frac{8}{8}=1
$$


and that for functional dependencies as

$$
\operatorname{sound}_{\Sigma^{t}}^{f}\left(\Sigma_{2}^{2}\right)=\frac{31}{31}=1 .
$$

For the completeness of uniqueness constraints we can easily observe that all meaningful uniqueness constraints have now been covered, that is,

$$
\text { complete }_{\Sigma^{t}}^{u}\left(\Sigma_{2}^{2}\right)=\frac{8}{8}=1
$$

and for functional dependencies there is just one attribute set closure that was not captured:

$$
\text { complete }_{\Sigma^{t}}^{f}\left(\Sigma_{2}^{2}\right)=\frac{30}{31} \simeq 0.97 .
$$

Again, as the soundness is 1, completeness and proximity measures coincide:

- $\operatorname{prox}^{u}\left(\Sigma_{2}^{2}, \Sigma^{t}\right)=\frac{8}{8}=1$,

- $\operatorname{prox}_{\Sigma^{t}}^{f}\left(\Sigma_{2}^{2}\right)=\frac{30}{31} \simeq 0.97$.

\section{Gain}

We show next the gain measures corresponding to the constraints specified by team 2 .

Indeed, team 2 achieved the following gains for uniqueness constraints:

- gain-in-sound ${ }_{2}^{u}=1-1=0$,

- gain-in-complete $e_{2}^{u}=1-0.5=0.5$, and

- gain-in-prox ${ }_{2}^{u}=1-0.5=0.5$,

and for functional dependencies as follows:

- gain-in-sound ${ }_{2}^{f}=$ gain-in-sound-for-FDs $=1-1=0$, 
- gain-in-complete ${ }_{2}^{f}=$ gain-in-complete-for-FDs $=0.97-0.81=0.16$,

- gain-in-prox $2_{2}^{f}=$ gain-in-prox-for-FDs $=0.97-0.81=0.16$.

This means that team 2 achieved a gain in soundness for NUCs by 0 percent, a gain in completeness for NUCs by 50 percent, a gain in proximity for NUCs by 50. Meanwhile, team 2 obtained a gain in soundness for NFDs by 0 percent, a gain in completeness for NFDs by 16 percent, a gain in proximity for NFDs by 16.

\section{Actual and possible gain}

The actual gains for team 2 can be summarized as follows:

- actual-gain-in-sound ${ }_{2}^{u}=$ gain-in-sound ${ }_{2}^{u}=0$,

- actual-gain-in-sound ${ }_{2}^{f}=$ gain-in-sound ${ }_{2}^{f}=0$,

- actual-gain-in-complete $_{2}^{u}=$ gain-in-complete ${ }_{2}^{u}=0.5$,

- actual-gain-in-complete $e_{2}^{f}=$ gain-in-complete $_{2}^{f}=0.16$,

- actual-gain-in-prox $x_{2}^{u}=$ gain-in-complete $e_{2}^{u}=0.5$,

- actual-gain-in-prox ${ }_{2}^{f}=$ gain-in-complete ${ }_{2}^{f}=0.16$.

These are best viewed in contrast to the possible gains for team 2:

- possible-gain-in-sound ${ }_{2}^{u}=1-1=0$,

- possible-gain-in-sound ${ }_{2}^{f}=1-1=0$,

- possible-gain-in-complete $_{2}^{u}=1-0.5=0.5$,

- possible-gain-in-complete $_{2}^{f}=1-0.8=0.2$,

- possible-gain-in-prox $x_{2}^{u}=1-0.5=0.5$,

- possible-gain-in-prox ${ }_{2}^{f}=1-0.8=0.2$. 
Remarkably, team 2 almost actually achieved what they could have possibly achieved in terms of soundness, completeness, and proximity. In terms of soundness, no improvement was possible but no decrease occurred neither.

\subsection{Further considerations and future research}

Some factors that may influence the results of the experiments include:

- students acting as database designers,

- familiarity of students with application domain,

- fixation of the null-free subschema,

- number and size of the application domain,

- time constraints,

- assumption that domain experts are present, and

- assumption that there is consensus among domain experts.

The argument that students are not database designers is certainly a valid one, in particular with respect to experience and their communication skills with the domain experts. However, it is by no means obvious that a database designer is more skilled or motivated to identify semantically meaningful constraints. For example, the experience of a designer could suggest that it is only worth to identify the primary key [94]. Therefore, experienced designers may not have an advantage after all in the task of identifying a cover of all semantically meaningful NUCs and NFDs. The lack of experience in students might contribute to be more open-minded or motivated to complete the given task. Also, if Armstrong tables are already helpful for unexperienced students, then they are certainly helpful 
to experienced database designers. For these reasons we found that students with a solid education in database concepts represent a reasonable choice for the design teams. Moreover, database designers may not be skilled at all with the interaction of constraints over partial tables.

A concern might be raised about the database designers' level of familiarity with the application domain. In practice, however, it may also be the case that designers are chosen that have some knowledge about the domain of interest. Of course, this is not always possible. It will be pointed out to the students that they cannot make assumptions about the domain, and that it is safer to consult with the domain experts. Let us suppose that the degree of familiarity was higher than in a "real-world" situation. Consequently, with a normal degree of familiarity the design teams would have specified a larger number of semantically meaningless constraints and a smaller number of semantically meaningful constraints prior to inspecting an Armstrong table. Consequently, the impact of Armstrong tables would be even bigger. Intuitively, this makes sense: the less one knows about the application domain, the more assistance a good sample table can provide.

In practice, design teams will also face the challenge to identify the null-free subschema of the application domain. In the experiment, the NFS was provided to the design teams. The reason is our intended focus on the uniqueness constraints and functional dependencies. Surely, if Armstrong tables are found useful for their discovery, then they will be at least as useful in case that the null-free subschema must be discovered as well.

Another concern is that we only consider one application domain, and this domain has at most five attributes. In future work, experiments should be conducted with different application domains. Our simple experiment will hopefully be illustrative enough to show how our measures can be applied to collect more evidence. The specific results and percentages that we report for our experiment are likely to vary for different domains. However, we strongly believe that the conclusion and overall insight will 
remain similar. Regarding the size of our application, we believe that the impact of Armstrong tables will be even more positive for schemas with more attributes. The reason is that the number of constraints to consider grows exponentially with the number of attributes. Therefore, it becomes more difficult to discover semantically meaningful constraints and to eliminate meaningless ones. We suspect that the usefulness of Armstrong tables will decline when their size becomes too large.

The experiment was conducted under stricter time constraints than potentially available in practice. However, the complexity of the underlying application domain gave the design teams sufficient time for consulting the domain experts, discuss their understandings and formulate their solutions.

In practice, it happens quite often that domain experts are unavailable or non-existent. We believe that in these cases Armstrong tables might be even more useful. Intuitively, Armstrong tables can pinpoint the decision whether a constraint is meaningful or not. When in doubt it is likely that the designers will not specify a constraint in order to guarantee that meaningful tables can still become database instances. That is, Armstrong tables may prevent the database designer to make assumptions about the application domain.

Furthermore, it is likely that there is no complete consensus among the domain experts, in particular not about the semantic meaningfulness of some constraint. Here we believe that Armstrong tables can help pinpoint the inconsistencies between the domain experts' opinions. A more complex experiment could leave the meaningfulness of some of the possible constraints open. In that case, several target FD sets would co-exist, and the measures would have to be generalized. One possibility would be to measure the quality of a constraint set relative to all possible target FD sets, and then to take an optimum. This issue, however, is beyond the scope of this study. 


\section{Chapter 7}

\section{Evaluation - Part II: Data Analysis}

This chapter presents the data analysis of the experiments on the usefulness of Armstrong tables for the acquisition of meaningful SQL constraints. In the quantitative analysis of Section 7.1 the measures, introduced in Chapter 6, are applied to the results collected from the 50 design teams. In Section 7.2 the measures of soundness, completeness and proximity are applied to the data from a qualitative point of view, revealing which meaningless and which meaningful constraints were discovered and discarded with the use of Armstrong tables. A summary of the findings for this chapter is presented in Section 7.3. Results on the quantitative findings of this chapter were announced in the conference paper [81].

\subsection{Quantitative data analysis}

Recall the different measures of soundness, completeness, and proximity that were proposed in Chapter 6 to assess the quality of a constraint set $\Sigma$ with respect to a target set $\Sigma^{t}$. As $\Sigma$ can consist of both uniqueness constraints and functional dependencies, each of the three measures above comes in two flavors: one for each type of constraint. Note, however, that all mea- 
sures are determined by both classes of constraints since uniqueness constraints imply functional dependencies, and uniqueness constraints and functional dependencies together determine other uniqueness constraints. This section contains a quantitative analysis of the data collected in the experiments for both flavors of each of the three measures.

\subsubsection{Quantitative analysis for soundness}

Recall that soundness measures which of the constraints perceived meaningful by a design team are actually meaningful.

\section{Soundness for UCs}

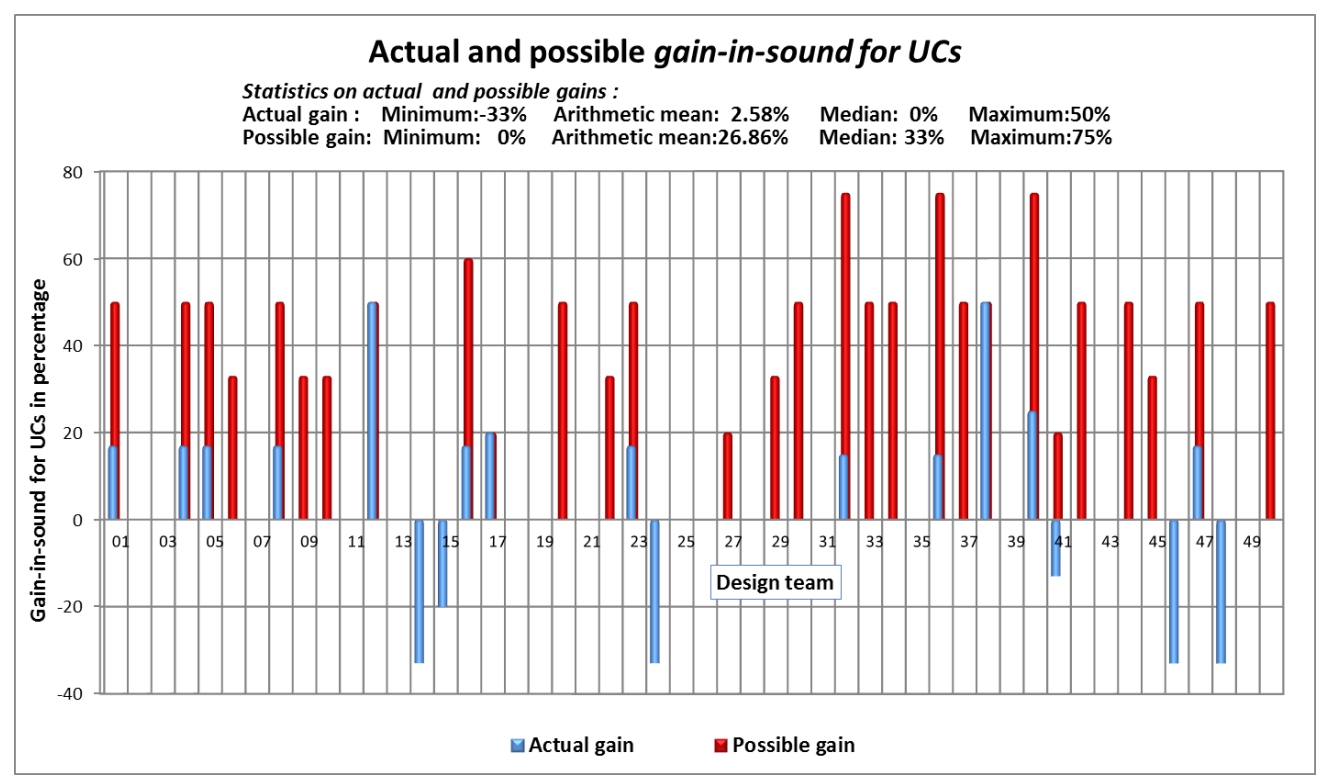

Figure 7.1: Possible and actual gain-in-sound for UCs

Table 7.1 shows the results for the soundness measures as they apply to uniqueness constraints for each of the 50 design teams that participated 


\begin{tabular}{|c|c|c|c|c|}
\hline Team $i$ & $\operatorname{sound}_{\Sigma^{t}}^{u}\left(\Sigma_{1}^{i}\right)$ & $\operatorname{sound}_{\Sigma^{t}}^{u}\left(\Sigma_{2}^{i}\right)$ & gain-in-sound $_{i}^{u}$ & possible-gain-in-sound ${ }_{i}^{u}$ \\
\hline 01 & 50 & 67 & 17 & 50 \\
\hline 02 & 100 & 100 & 0 & 0 \\
\hline 03 & 100 & 100 & 0 & 0 \\
\hline 04 & 50 & 67 & 17 & 50 \\
\hline 05 & 50 & 67 & 17 & 50 \\
\hline 06 & 67 & 67 & 0 & 33 \\
\hline 07 & 100 & 100 & 0 & 0 \\
\hline 08 & 50 & 67 & 17 & 50 \\
\hline 09 & 67 & 67 & 0 & 33 \\
\hline 10 & 67 & 67 & 0 & 33 \\
\hline 11 & 100 & 100 & 0 & 0 \\
\hline 12 & 50 & 100 & 50 & 50 \\
\hline 13 & 100 & 100 & 0 & 0 \\
\hline 14 & 100 & 67 & -33 & 0 \\
\hline 15 & 100 & 80 & -20 & 0 \\
\hline 16 & 40 & 57 & 17 & 60 \\
\hline 17 & 80 & 100 & 20 & 20 \\
\hline 18 & 100 & 100 & 0 & 0 \\
\hline 19 & 100 & 100 & 0 & 0 \\
\hline 20 & 50 & 50 & 0 & 50 \\
\hline 21 & 100 & 100 & 0 & 0 \\
\hline 22 & 67 & 67 & 0 & 33 \\
\hline 23 & 50 & 67 & 17 & 50 \\
\hline 24 & 100 & 67 & -33 & 0 \\
\hline 25 & 100 & 100 & 0 & 0 \\
\hline 26 & 100 & 100 & 0 & 0 \\
\hline 27 & 80 & 80 & 0 & 20 \\
\hline 28 & 100 & 100 & 0 & 0 \\
\hline 29 & 67 & 67 & 0 & 33 \\
\hline 30 & 50 & 50 & 0 & 50 \\
\hline 31 & 100 & 100 & 0 & 0 \\
\hline 32 & 25 & 40 & 15 & 75 \\
\hline 33 & 50 & 50 & 0 & 50 \\
\hline 34 & 50 & 50 & 0 & 50 \\
\hline 35 & 100 & 100 & 0 & 0 \\
\hline 36 & 25 & 40 & 15 & 75 \\
\hline 37 & 50 & 50 & 0 & 50 \\
\hline 38 & 50 & 100 & 50 & 50 \\
\hline 39 & 100 & 100 & 0 & 0 \\
\hline 40 & 25 & 50 & 25 & 75 \\
\hline 41 & 80 & 67 & -13 & 20 \\
\hline 42 & 50 & 50 & 0 & 50 \\
\hline 43 & 100 & 100 & 0 & 0 \\
\hline 44 & 50 & 50 & 0 & 50 \\
\hline 45 & 67 & 67 & 0 & 33 \\
\hline 46 & 100 & 67 & -33 & 0 \\
\hline 47 & 50 & 67 & 17 & 50 \\
\hline 48 & 100 & 67 & -33 & 0 \\
\hline 49 & 100 & 100 & 0 & 0 \\
\hline 50 & 50 & 50 & 0 & 50 \\
\hline Average & 73.1 & 75.7 & 2.6 & 26.9 \\
\hline
\end{tabular}

Table 7.1: Soundness measures for uniqueness constraints in percent 
in the experiments. The first two columns show the soundness that each team scored in phase 1 (that is, before inspecting Armstrong tables) and phase 2 (that is, after inspecting Armstrong tables) with respect to the target set. The next column displays the (actual) gains in soundness achieved by the teams. The last column shows the possible gain as possible improvement each team could have potentially achieved with the help of Armstrong tables. Along with Table 7.1. Figure 7.1 visualizes differences between possible and actual gains in soundness for uniqueness constraints for each of the teams, and shows some basic statistics.

The main observation from Table 7.1 is that Armstrong tables should not be considered useful in terms of soundness for uniqueness constraints. That is, it is unlikely that, by the inspection of Armstrong tables, teams will recognize actually meaningless uniqueness constraints that they perceived meaningful prior to the inspection of Armstrong tables. Indeed, our quantitative data analysis shows a mere $2.6 \%$ average gain (i.e. arithmetic mean) in soundness. After inspecting Armstrong tables, around one fourth of the teams achieved an actual improvement in soundness (that is, teams $1,4,5,8,12,16,17,23,32,36,38,40$, and 47). Worse than that, six teams (that is, teams $14,15,24,41,46$, and 48 ) had some losses (that is, a negative gain in soundness). The highest loss was $33 \%$ by four teams 14 , 24,46 , and 48 . The greatest gain at $50 \%$ was achieved by both teams 12 and 38. Only three teams (that is, teams 12,17 , and 38) achieved a significant increase in soundness for uniqueness constraints and attained the highest possible score of $100 \%$.

Figure 7.1 illustrates the minor impact of Armstrong tables on the soundness of uniqueness constraints even better. It is easy to observe the significant difference between the actual and possible gains in soundness for uniqueness constraints. While possible gains average around $26.9 \%$, the actual gains only average around $2.6 \%$. Around one third of the 50 teams meet their possible gains with the help of Armstrong tables (that is, teams $2,3,7,11,12,13,17,18,19,21,25,26,28,31,35,38,39,43$, and 49), but 


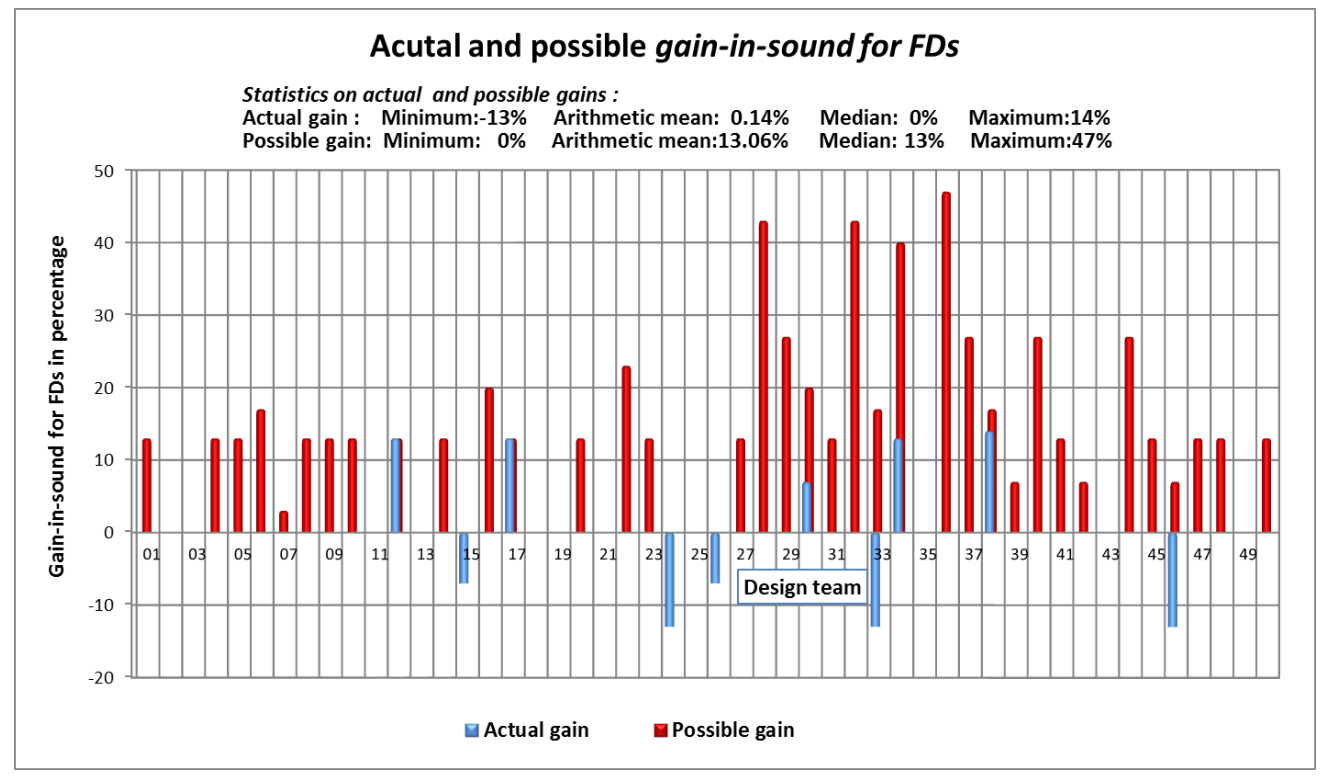

Figure 7.2: Possible and actual gain-in-sound for FDs

for 16 out of those 19 teams (namely, teams 2, 3, 7, 11, 13, 18, 19, 21, 25, 26, $28,31,35,39,43$, and 49 ) the possible gain was $0 \%$. The greatest difference between two sets of gains is $60 \%$, achieved by both teams 32 and 36 .

In summary, by inspecting Armstrong tables database designers are unlikely to recognize actually meaningless uniqueness constraints that they incorrectly perceive as meaningful prior to the inspection. Indeed, the satisfaction of meaningless uniqueness constraints is nearly impossible to be observed.

\section{Soundness for FDs}

Table 7.2 shows the results for the soundness measures as they apply to functional dependencies for each of the 50 design teams that participated in the experiments. The first two columns show the soundness that each team scored in phase 1 (that is, before inspecting Armstrong tables) 


\begin{tabular}{|c|c|c|c|c|}
\hline Team $i$ & $\operatorname{sound}_{\Sigma^{t}}^{f}\left(\Sigma_{1}^{i}\right)$ & $\operatorname{sound}_{\Sigma^{t}}^{f}\left(\Sigma_{2}^{i}\right)$ & gain-in-sound $i_{i}^{f}$ & possible-gain-in-sound ${ }_{i}^{f}$ \\
\hline 01 & 87 & 87 & 0 & 13 \\
\hline 02 & 100 & 100 & 0 & 0 \\
\hline 03 & 100 & 100 & 0 & 0 \\
\hline 04 & 87 & 87 & 0 & 13 \\
\hline 05 & 87 & 87 & 0 & 13 \\
\hline 06 & 83 & 83 & 0 & 17 \\
\hline 07 & 97 & 97 & 0 & 3 \\
\hline 08 & 87 & 87 & 0 & 13 \\
\hline 09 & 87 & 87 & 0 & 13 \\
\hline 10 & 87 & 87 & 0 & 13 \\
\hline 11 & 100 & 100 & 0 & 0 \\
\hline 12 & 87 & 100 & 13 & 13 \\
\hline 13 & 100 & 100 & 0 & 0 \\
\hline 14 & 87 & 87 & 0 & 13 \\
\hline 15 & 100 & 93 & -7 & 0 \\
\hline 16 & 80 & 80 & 0 & 20 \\
\hline 17 & 87 & 100 & 13 & 13 \\
\hline 18 & 100 & 100 & 0 & 0 \\
\hline 19 & 100 & 100 & 0 & 0 \\
\hline 20 & 87 & 87 & 0 & 13 \\
\hline 21 & 100 & 100 & 0 & 0 \\
\hline 22 & 77 & 77 & 0 & 23 \\
\hline 23 & 87 & 87 & 0 & 13 \\
\hline 24 & 100 & 87 & -13 & 0 \\
\hline 25 & 100 & 100 & 0 & 0 \\
\hline 26 & 100 & 93 & -7 & 0 \\
\hline 27 & 87 & 87 & 0 & 13 \\
\hline 28 & 57 & 57 & 0 & 43 \\
\hline 29 & 73 & 73 & 0 & 27 \\
\hline 30 & 80 & 87 & 7 & 20 \\
\hline 31 & 87 & 87 & 0 & 13 \\
\hline 32 & 57 & 57 & 0 & 43 \\
\hline 33 & 83 & 70 & -13 & 17 \\
\hline 34 & 60 & 73 & 13 & 40 \\
\hline 35 & 100 & 100 & 0 & 0 \\
\hline 36 & 53 & 53 & 0 & 47 \\
\hline 37 & 73 & 73 & 0 & 27 \\
\hline 38 & 83 & 97 & 14 & 17 \\
\hline 39 & 93 & 93 & 0 & 7 \\
\hline 40 & 73 & 73 & 0 & 27 \\
\hline 41 & 87 & 87 & 0 & 13 \\
\hline 42 & 93 & 93 & 0 & 7 \\
\hline 43 & 100 & 100 & 0 & 0 \\
\hline 44 & 73 & 73 & 0 & 27 \\
\hline 45 & 87 & 87 & 0 & 13 \\
\hline 46 & 93 & 80 & -13 & 7 \\
\hline 47 & 87 & 87 & 0 & 13 \\
\hline 48 & 87 & 87 & 0 & 13 \\
\hline 49 & 100 & 100 & 0 & 0 \\
\hline 50 & 87 & 87 & 0 & 13 \\
\hline Average & 86.94 & 87.08 & 0.1 & 13.1 \\
\hline
\end{tabular}

Table 7.2: Soundness measures for FDs in percent 
and phase 2 (that is, after inspecting Armstrong tables) with respect to the target set. The next column displays the (actual) gains in soundness achieved by the teams. The last column shows the possible gain as possible improvement each team could have potentially achieved with the help of Armstrong tables. Along with Table 7.2, Figure 7.2 visualizes differences between possible and actual gains in soundness for functional dependencies for each of the teams, and shows some basic statistics.

The main observation from Table 7.2 is that Armstrong tables should not be considered useful in terms of soundness for functional dependencies. That is, it is unlikely that, by the inspection of Armstrong tables, teams will recognize actually meaningless functional dependencies that they perceived meaningful prior to the inspection of Armstrong tables. Indeed, our quantitative data analysis shows a mere $0.1 \%$ average gain (i.e. arithmetic mean) in soundness for functional dependencies. In fact, only five out of 50 teams (namely, teams 12, 17, 30,34, and 38) achieve any gains in soundness. Five other teams (namely, teams 15, 24, 26, 33 and 46) even drop in soundness after inspecting Armstrong tables (that is, they have a negative gain in soundness). The rest of the teams keep their soundness measures unchanged, and 11 of them (namely, teams 2, 3, 11, 13, 18, 19, 21, $25,35,43$, and 49) maintain the highest possible score of $100 \%$.

Figure 7.2 illustrates the minor impact of Armstrong tables on the soundness of functional dependencies even better. It is easy to observe the significant difference between the actual and possible gains in soundness for uniqueness constraints. While possible gains average around $13.1 \%$, the actual gains are next to zero, that is $0.1 \%$. Indeed, only two of the 50 teams (namely, teams 12 and 17) were able to actually meet their possible non-zero gains. The widest gap between the actual and possible gains in soundness for functional dependencies is $47 \%$ for team 36 , followed by a gap of $43 \%$ for teams 28 and 32. Gaps for other teams range from 3\% to $30 \%$.

In summary, we can extend our previous observation on the sound- 
ness from uniqueness constraints to functional dependencies. That is, by inspecting Armstrong tables database designers are unlikely to recognize actually meaningless functional dependencies that they incorrectly perceive as meaningful prior to the inspection. Indeed, the satisfaction of meaningless functional dependencies is nearly impossible to be observed.

\subsubsection{Quantitative analysis for completeness}

This subsection continues the quantitative analysis of the data with the completeness measures introduced in Chapter 6. Recall that completeness measures which of the actually meaningful constraints are also perceived as meaningful by a team.

\section{Completeness for UCs}

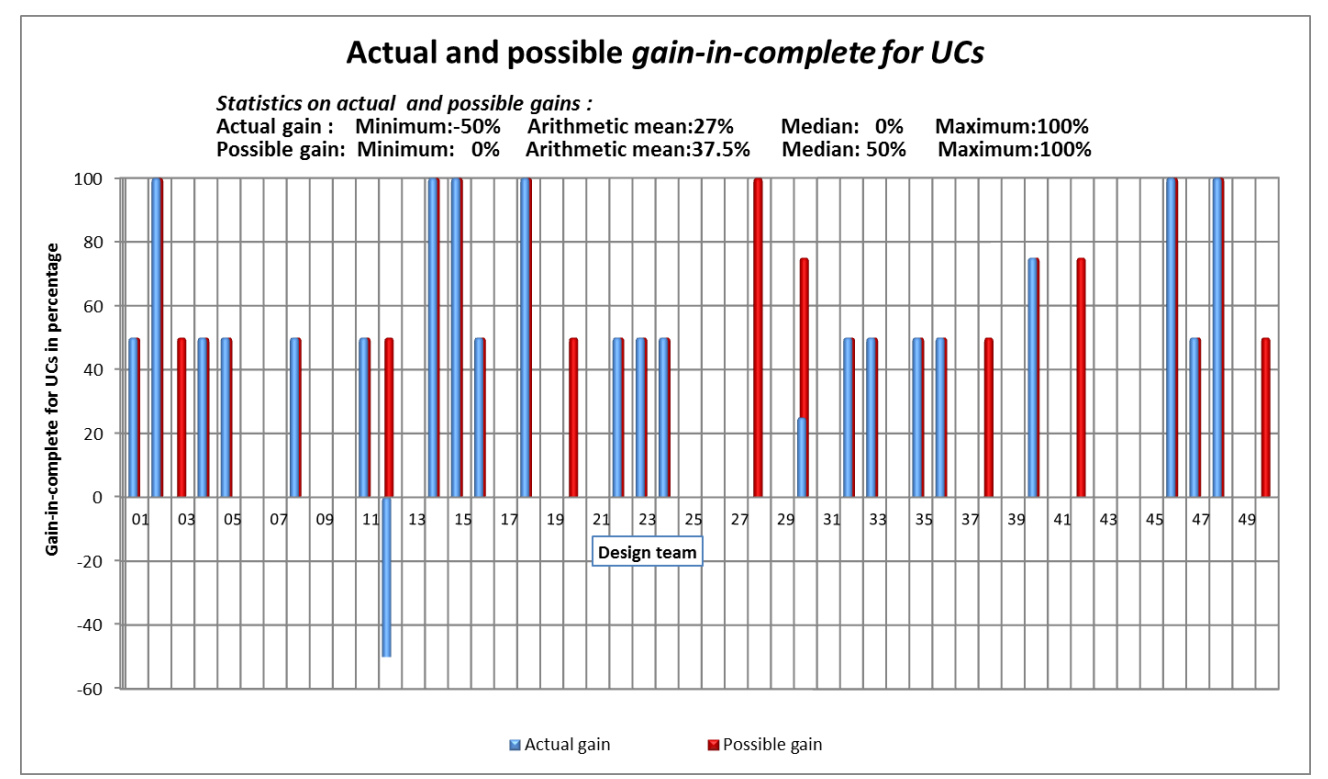

Figure 7.3: Possible and actual gain-in-complete for UCs 


\begin{tabular}{|c|c|c|c|c|}
\hline Team $i$ & $\operatorname{complete}_{\Sigma^{t}}^{u}\left(\Sigma_{1}^{i}\right)$ & $\operatorname{complete}_{\Sigma^{t}}^{u}\left(\Sigma_{2}^{i}\right)$ & gain-in-complete $i_{i}^{u}$ & possible-gain-in-complete $_{i}^{u}$ \\
\hline 01 & 50 & 100 & 50 & 50 \\
\hline 02 & 0 & 100 & 100 & 100 \\
\hline 03 & 50 & 50 & 0 & 50 \\
\hline 04 & 50 & 100 & 50 & 50 \\
\hline 05 & 50 & 100 & 50 & 50 \\
\hline 06 & 100 & 100 & 0 & 0 \\
\hline 07 & 100 & 100 & 0 & 0 \\
\hline 08 & 50 & 100 & 50 & 50 \\
\hline 09 & 100 & 100 & 0 & 0 \\
\hline 10 & 100 & 100 & 0 & 0 \\
\hline 11 & 50 & 100 & 50 & 50 \\
\hline 12 & 50 & 0 & -50 & 50 \\
\hline 13 & 100 & 100 & 0 & 0 \\
\hline 14 & 0 & 100 & 100 & 100 \\
\hline 15 & 0 & 100 & 100 & 100 \\
\hline 16 & 50 & 100 & 50 & 50 \\
\hline 17 & 100 & 100 & 0 & 0 \\
\hline 18 & 0 & 100 & 100 & 100 \\
\hline 19 & 100 & 100 & 0 & 0 \\
\hline 20 & 50 & 50 & 0 & 50 \\
\hline 21 & 100 & 100 & 0 & 0 \\
\hline 22 & 50 & 100 & 50 & 50 \\
\hline 23 & 50 & 100 & 50 & 50 \\
\hline 24 & 50 & 100 & 50 & 50 \\
\hline 25 & 100 & 100 & 0 & 0 \\
\hline 26 & 100 & 100 & 0 & 0 \\
\hline 27 & 100 & 100 & 0 & 0 \\
\hline 28 & 0 & 0 & 0 & 100 \\
\hline 29 & 100 & 100 & 0 & 0 \\
\hline 30 & 25 & 50 & 25 & 75 \\
\hline 31 & 100 & 100 & 0 & 0 \\
\hline 32 & 50 & 100 & 50 & 50 \\
\hline 33 & 50 & 100 & 50 & 50 \\
\hline 34 & 100 & 100 & 0 & 0 \\
\hline 35 & 50 & 100 & 50 & 50 \\
\hline 36 & 50 & 100 & 50 & 50 \\
\hline 37 & 100 & 100 & 0 & 0 \\
\hline 38 & 50 & 50 & 0 & 50 \\
\hline 39 & 100 & 100 & 0 & 0 \\
\hline 40 & 25 & 100 & 75 & 75 \\
\hline 41 & 100 & 100 & 0 & 0 \\
\hline 42 & 25 & 25 & 0 & 75 \\
\hline 43 & 100 & 100 & 0 & 0 \\
\hline 44 & 100 & 100 & 0 & 0 \\
\hline 45 & 100 & 100 & 0 & 0 \\
\hline 46 & 0 & 100 & 100 & 100 \\
\hline 47 & 50 & 100 & 50 & 50 \\
\hline 48 & 0 & 100 & 100 & 100 \\
\hline 49 & 100 & 100 & 0 & 0 \\
\hline 50 & 50 & 50 & 0 & 50 \\
\hline Average & 62.5 & 89.5 & 27 & 37.5 \\
\hline
\end{tabular}

Table 7.3: Completeness measures for UCs in percent 
Table 7.3 shows the results for the completeness measures as they apply to uniqueness constraints for each of the 50 design teams that participated in the experiments. The first two columns show the completeness that each team scored in phase 1 (that is, before inspecting Armstrong tables) and phase 2 (that is, after inspecting Armstrong tables) with respect to the target set. The next column displays the (actual) gains in completeness achieved by the teams. The last column shows the possible gain as possible improvement each team could have potentially achieved with the help of Armstrong tables. Along with Table 7.3, Figure 7.3 visualizes differences between possible and actual gains in completeness for uniqueness constraints for each of the teams, and shows some basic statistics.

The main observation from Table 7.3 is that Armstrong tables should be considered useful in terms of completeness for uniqueness constraints. That is, it is likely that, by the inspection of Armstrong tables, teams will recognize actually meaningful uniqueness constraints that they perceived meaningless prior to the inspection of Armstrong tables. Indeed, our quantitative data analysis shows a remarkable $27 \%$ average gain (i.e. arithmetic mean) in completeness for uniqueness constraints. Indeed, most of the teams achieved the highest possible score of $100 \%$ while among the remaining eight teams (that is, teams $3,12,20,28,30,38,42$, and 50), six still maintained their scores and only team 12 had a negative gain. The inspection of Armstrong tables also assists 21 teams (that is, teams 6, 7, 9, 10, 13, $17,19,21,25,26,27,29,31,34,37,39,41,43,44,45$, and 49) in maintaining the highest completeness score possible.

Figure 7.3 illustrates the big impact of Armstrong tables on the completeness of uniqueness constraints even better. It is easy to observe the minor differences between the actual and possible gains in completeness for uniqueness constraints. While possible gains average around $37.5 \%$, the actual gains average around $27 \%$. Indeed, 42 out of 50 teams actually met the possible gains in completeness for uniqueness constraints while other teams (that is, teams $3,12,20,28,30,38,42$, and 50) have gaps be- 
tween actual and possible gains that range from 50\% to $100 \%$.

In summary, by inspecting Armstrong tables it is likely for database designers to recognize meaningful uniqueness constraints that they incorrectly perceive as meaningless prior to the inspection. Indeed, the violation of meaningful uniqueness constraints is rather certain to be observed in Armstrong tables.

\section{Completeness for FDs}

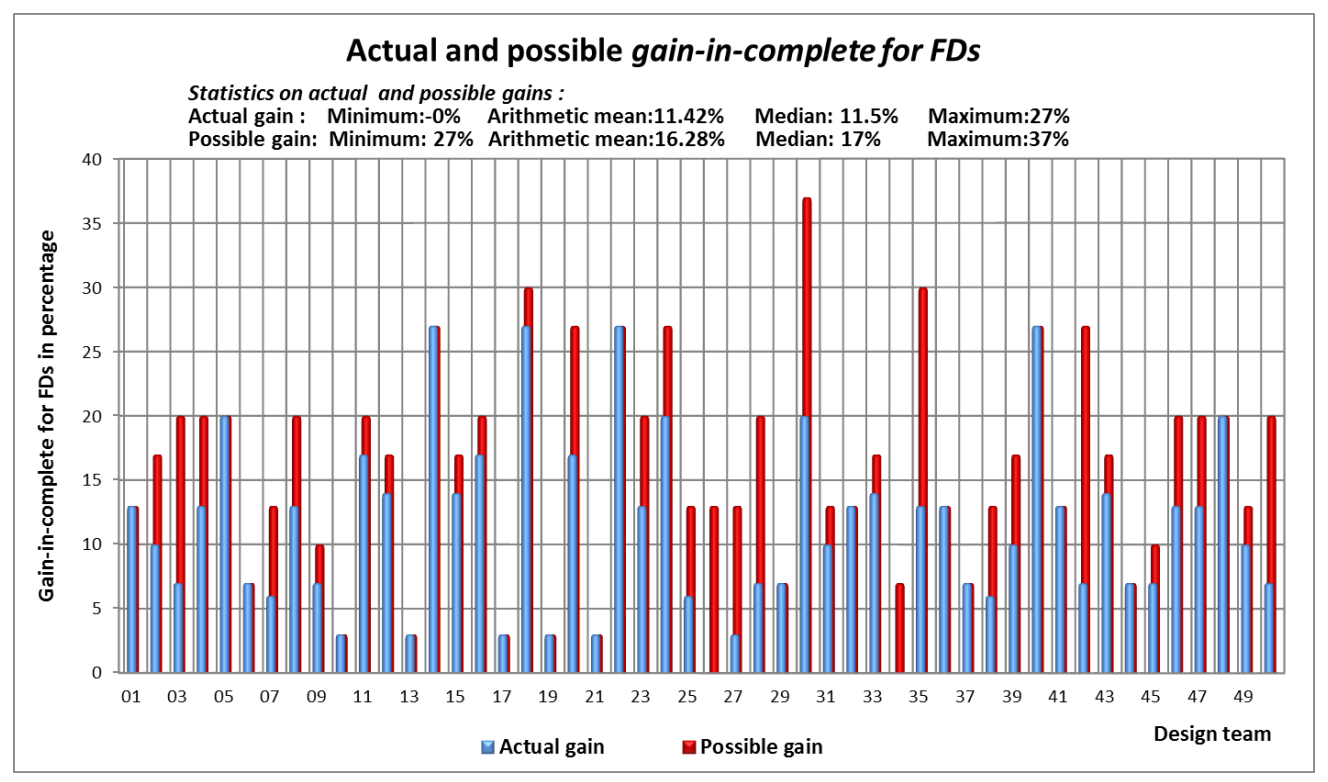

Figure 7.4: Possible and actual gain-in-complete for FDs

Table 7.4 shows the results for the completeness measures as they apply to functional dependencies for each of the 50 design teams that participated in the experiments. The first two columns show the completeness that each team scored in phase 1 (that is, before inspecting Armstrong tables) and phase 2 (that is, after inspecting Armstrong tables) with respect 


\begin{tabular}{|c|c|c|c|c|}
\hline Team $i$ & complete $_{\Sigma^{t}}^{f}\left(\Sigma_{1}^{i}\right)$ & complete $_{\Sigma^{t}}^{f}\left(\Sigma_{2}^{i}\right)$ & gain-in-complete ${ }_{i}^{f}$ & possible-gain-in-complete ${ }_{i}^{f}$ \\
\hline 01 & 87 & 100 & 13 & 13 \\
\hline 02 & 83 & 93 & 10 & 17 \\
\hline 03 & 80 & 87 & 7 & 20 \\
\hline 04 & 80 & 93 & 13 & 20 \\
\hline 05 & 80 & 100 & 20 & 20 \\
\hline 06 & 93 & 100 & 7 & 7 \\
\hline 07 & 87 & 93 & 6 & 13 \\
\hline 08 & 80 & 93 & 13 & 20 \\
\hline 09 & 90 & 97 & 7 & 10 \\
\hline 10 & 97 & 100 & 3 & 3 \\
\hline 11 & 80 & 97 & 17 & 20 \\
\hline 12 & 83 & 97 & 14 & 17 \\
\hline 13 & 97 & 100 & 3 & 3 \\
\hline 14 & 73 & 100 & 27 & 27 \\
\hline 15 & 83 & 97 & 14 & 17 \\
\hline 16 & 80 & 97 & 17 & 20 \\
\hline 17 & 97 & 100 & 3 & 3 \\
\hline 18 & 70 & 97 & 27 & 30 \\
\hline 19 & 97 & 100 & 3 & 3 \\
\hline 20 & 73 & 90 & 17 & 27 \\
\hline 21 & 97 & 100 & 3 & 3 \\
\hline 22 & 73 & 100 & 27 & 27 \\
\hline 23 & 80 & 93 & 13 & 20 \\
\hline 24 & 73 & 93 & 20 & 27 \\
\hline 25 & 87 & 93 & 6 & 13 \\
\hline 26 & 87 & 87 & 0 & 13 \\
\hline 27 & 87 & 90 & 3 & 13 \\
\hline 28 & 80 & 87 & 7 & 20 \\
\hline 29 & 93 & 100 & 7 & 7 \\
\hline 30 & 63 & 83 & 20 & 37 \\
\hline 31 & 87 & 97 & 10 & 13 \\
\hline 32 & 87 & 100 & 13 & 13 \\
\hline 33 & 83 & 97 & 14 & 17 \\
\hline 34 & 93 & 93 & 0 & 7 \\
\hline 35 & 70 & 83 & 13 & 30 \\
\hline 36 & 87 & 100 & 13 & 13 \\
\hline 37 & 93 & 100 & 7 & 7 \\
\hline 38 & 87 & 93 & 6 & 13 \\
\hline 39 & 83 & 93 & 10 & 17 \\
\hline 40 & 73 & 100 & 27 & 27 \\
\hline 41 & 87 & 100 & 13 & 13 \\
\hline 42 & 73 & 80 & 7 & 27 \\
\hline 43 & 83 & 97 & 14 & 17 \\
\hline 44 & 93 & 100 & 7 & 7 \\
\hline 45 & 90 & 97 & 7 & 10 \\
\hline 46 & 80 & 93 & 13 & 20 \\
\hline 47 & 80 & 93 & 13 & 20 \\
\hline 48 & 80 & 100 & 20 & 20 \\
\hline 49 & 87 & 97 & 10 & 13 \\
\hline 50 & 80 & 87 & 7 & 20 \\
\hline Average & 83.7 & 95.1 & 11.4 & 16.3 \\
\hline
\end{tabular}

Table 7.4: Completeness measures for FDs in percent 
to the target set. The next column displays the (actual) gains in completeness achieved by the teams. The last column shows the possible gain as possible improvement each team could have potentially achieved with the help of Armstrong tables. Along with Table 7.4. Figure 7.4 visualizes differences between possible and actual gains in completeness for functional dependencies for each of the teams, and shows some basic statistics.

The main observation from Table 7.4 is that Armstrong tables should be considered useful in terms of completeness for functional dependencies. That is, it is likely that, by the inspection of Armstrong tables, teams will recognize actually meaningful functional dependencies that they perceived meaningless prior to the inspection of Armstrong tables. Indeed, our quantitative data analysis shows a sizeable $11.4 \%$ average gain (i.e. arithmetic mean) in completeness for functional dependencies. Apart from teams 26 and 34, which do not change their completeness scores, all teams achieve gains that range between $3 \%$ and $27 \%$. While no team achieved the highest possible completeness score of $100 \%$ in phase 1,18 teams (that is, teams $1,5,6,10,13,14,17,19,21,22,29,32,36,37,40,41,44$, and 48) achieved the top score in phase 2 .

Figure 7.4 illustrates the big impact of Armstrong tables on the completeness of functional dependencies even better. It is easy to observe the minor differences between the actual and possible gains in completeness for functional dependencies. While possible gains average around $16.3 \%$, the actual gains average around $11.4 \%$. Indeed, 19 out of 50 teams actually met the possible gains in completeness for functional dependencies (that is, teams $1,2,5,6,10,13,14,17,19,21,22,29,32,36,37,40,41,44$, and 47)

Hence, we can extend our previous observation on the completeness from uniqueness constraints to functional dependencies. That is, by inspecting Armstrong tables it is likely for database designers to recognize meaningful functional dependencies that they incorrectly perceive as meaningless prior to the inspection. Indeed, the violation of meaningful functional dependencies is rather certain to be observed in Armstrong tables. 


\subsubsection{Quantitative analysis for proximity}

This subsection continues the quantitative analysis of the data with the proximity measures introduced in Chapter 6. Recall that proximity measures how close the perception of a design team is to the target set of constraints. Proximity combines soundness and completeness. Intuitively, one would therefore expect an average behavior for the combination of the two.

\section{Proximity for UCs}

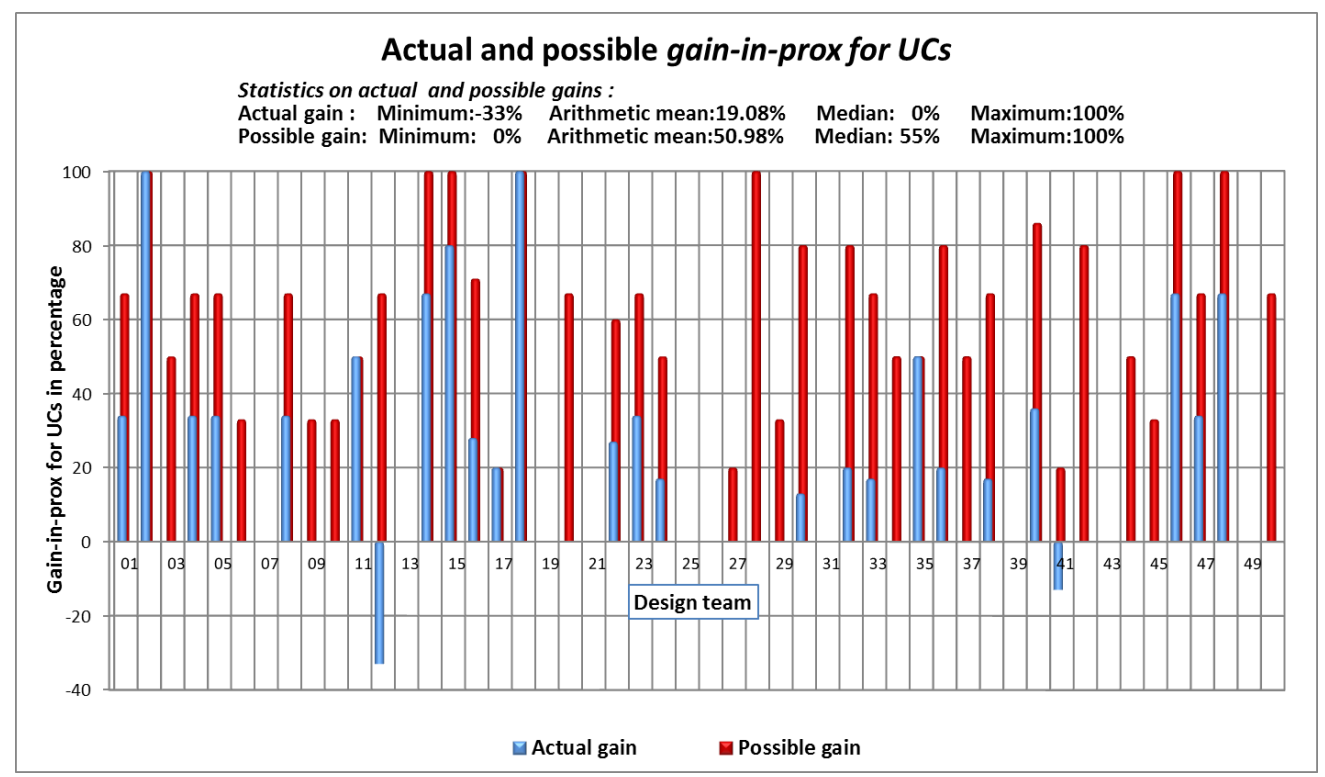

Figure 7.5: Possible and actual gain-in-prox for UCs

Table 7.5 shows the results for the proximity measures as they apply to uniqueness constraints for each of the 50 design teams that participated in the experiments. The first two columns show the proximity that each team scored in phase 1 (that is, before inspecting Armstrong tables) and phase 


\begin{tabular}{|c|c|c|c|c|}
\hline Team $i$ & $\operatorname{prox}_{\Sigma^{t}}^{u}\left(\Sigma_{1}^{i}\right)$ & $\operatorname{prox}_{\Sigma^{t}}^{u}\left(\Sigma_{2}^{i}\right)$ & gain-in-prox ${ }_{i}^{u}$ & possible-gain-in-prox $u$ \\
\hline 01 & 33 & 67 & 34 & 67 \\
\hline 02 & 0 & 100 & 100 & 100 \\
\hline 03 & 50 & 50 & 0 & 50 \\
\hline 04 & 33 & 67 & 34 & 67 \\
\hline 05 & 33 & 67 & 34 & 67 \\
\hline 06 & 67 & 67 & 0 & 33 \\
\hline 07 & 100 & 100 & 0 & 0 \\
\hline 08 & 33 & 67 & 34 & 67 \\
\hline 09 & 67 & 67 & 0 & 33 \\
\hline 10 & 67 & 67 & 0 & 33 \\
\hline 11 & 50 & 100 & 50 & 50 \\
\hline 12 & 33 & 0 & -33 & 67 \\
\hline 13 & 100 & 100 & 0 & 0 \\
\hline 14 & 0 & 67 & 67 & 100 \\
\hline 15 & 0 & 80 & 80 & 100 \\
\hline 16 & 29 & 57 & 28 & 71 \\
\hline 17 & 80 & 100 & 20 & 20 \\
\hline 18 & 0 & 100 & 100 & 100 \\
\hline 19 & 100 & 100 & 0 & 0 \\
\hline 20 & 33 & 33 & 0 & 67 \\
\hline 21 & 100 & 100 & 0 & 0 \\
\hline 22 & 40 & 67 & 27 & 60 \\
\hline 23 & 33 & 67 & 34 & 67 \\
\hline 24 & 50 & 67 & 17 & 50 \\
\hline 25 & 100 & 100 & 0 & 0 \\
\hline 26 & 100 & 100 & 0 & 0 \\
\hline 27 & 80 & 80 & 0 & 20 \\
\hline 28 & 0 & 0 & 0 & 100 \\
\hline 29 & 67 & 67 & 0 & 33 \\
\hline 30 & 20 & 33 & 13 & 80 \\
\hline 31 & 100 & 100 & 0 & 0 \\
\hline 32 & 20 & 40 & 20 & 80 \\
\hline 33 & 33 & 50 & 17 & 67 \\
\hline 34 & 50 & 50 & 0 & 50 \\
\hline 35 & 50 & 100 & 50 & 50 \\
\hline 36 & 20 & 40 & 20 & 80 \\
\hline 37 & 50 & 50 & 0 & 50 \\
\hline 38 & 33 & 50 & 17 & 67 \\
\hline 39 & 100 & 100 & 0 & 0 \\
\hline 40 & 14 & 50 & 36 & 86 \\
\hline 41 & 80 & 67 & -13 & 20 \\
\hline 42 & 20 & 20 & 0 & 80 \\
\hline 43 & 100 & 100 & 0 & 0 \\
\hline 44 & 50 & 50 & 0 & 50 \\
\hline 45 & 67 & 67 & 0 & 33 \\
\hline 46 & 0 & 67 & 67 & 100 \\
\hline 47 & 33 & 67 & 34 & 67 \\
\hline 48 & 0 & 67 & 67 & 100 \\
\hline 49 & 100 & 100 & 0 & 0 \\
\hline 50 & 33 & 33 & 0 & 67 \\
\hline Average & 49 & 68.1 & 19.1 & 51 \\
\hline
\end{tabular}

Table 7.5: Proximity measures for UCs in percent 
2 (that is, after inspecting Armstrong tables) with respect to the target set. The next column displays the (actual) gains in proximity achieved by the teams. The last column shows the possible gain as possible improvement each team could have potentially achieved with the help of Armstrong tables. Along with Table 7.5, Figure 7.5 visualizes differences between possible and actual gains in proximity for uniqueness constraints for each of the teams, and shows basic statistics.

The main observation from Table 7.5 is that Armstrong tables should be considered useful in terms of proximity for uniqueness constraints. That is, it is likely that, by the inspection of Armstrong tables, teams will improve the overall quality of the uniqueness constraints. Indeed, our quantitative data analysis shows a significant $19.1 \%$ average gain (i.e. arithmetic mean) in proximity for uniqueness constraints. The only teams with negative gains in proximity were teams 12 and 41 with a loss of $33 \%$ and $13 \%$, respectively. 24 out of the 50 teams had gains ranging from $13 \%$ up to $100 \%$ (namely, teams $1,2,4,5,8,11,14-18,22-24,30,32,33,35,36,38$, 40 , and $46-48)$. The remaining teams had no change in proximity.

Figure 7.5 illustrates the considerable impact of Armstrong tables on the proximity of uniqueness constraints even better. It is easy to observe that, on average, the actual gains are about two fifths of the possible gains in proximity for uniqueness constraints. In fact, possible gains average around $51 \%$ and actual gains average around $19.1 \%$. This behavior is consistent with that of soundness and completeness: soundness has almost no actual gains while actual and possible gains are nearly the same for completeness. About one third of the teams actually meet their possible gains (namely, teams 2, 7, 11, 13, 17-19, 21, 25, 26, 31, 35, 39, 43, 49).

In summary, by inspecting Armstrong tables it is likely for database designers to improve the overall quality of the uniqueness constraints that they acquire. 


\begin{tabular}{|c|c|c|c|c|}
\hline Team $i$ & $\operatorname{prox}_{\Sigma^{t}}^{f}\left(\Sigma_{1}^{i}\right)$ & $\operatorname{prox}_{\Sigma^{t}}^{f}\left(\Sigma_{2}^{i}\right)$ & gain-in-prox $i_{i}^{f}$ & possible-gain-in-prox $x_{i}^{f}$ \\
\hline 01 & 73 & 87 & 14 & 27 \\
\hline 02 & 83 & 93 & 10 & 17 \\
\hline 03 & 80 & 87 & 7 & 20 \\
\hline 04 & 67 & 80 & 13 & 33 \\
\hline 05 & 67 & 87 & 20 & 33 \\
\hline 06 & 77 & 83 & 6 & 23 \\
\hline 07 & 83 & 90 & 7 & 17 \\
\hline 08 & 67 & 80 & 13 & 33 \\
\hline 09 & 77 & 83 & 6 & 23 \\
\hline 10 & 83 & 87 & 4 & 17 \\
\hline 11 & 80 & 97 & 17 & 20 \\
\hline 12 & 70 & 97 & 27 & 30 \\
\hline 13 & 97 & 100 & 3 & 3 \\
\hline 14 & 67 & 87 & 20 & 33 \\
\hline 15 & 83 & 90 & 7 & 17 \\
\hline 16 & 60 & 77 & 17 & 40 \\
\hline 17 & 83 & 100 & 17 & 17 \\
\hline 18 & 70 & 97 & 27 & 30 \\
\hline 19 & 97 & 100 & 3 & 3 \\
\hline 20 & 60 & 77 & 17 & 40 \\
\hline 21 & 97 & 100 & 3 & 3 \\
\hline 22 & 57 & 77 & 20 & 43 \\
\hline 23 & 67 & 80 & 13 & 33 \\
\hline 24 & 73 & 80 & 7 & 27 \\
\hline 25 & 87 & 93 & 6 & 13 \\
\hline 26 & 87 & 80 & -7 & 13 \\
\hline 27 & 77 & 80 & 3 & 23 \\
\hline 28 & 37 & 43 & 6 & 63 \\
\hline 29 & 67 & 73 & 6 & 33 \\
\hline 30 & 43 & 70 & 27 & 57 \\
\hline 31 & 73 & 83 & 10 & 27 \\
\hline 32 & 43 & 57 & 14 & 57 \\
\hline 33 & 67 & 67 & 0 & 33 \\
\hline 34 & 53 & 67 & 14 & 47 \\
\hline 35 & 70 & 83 & 13 & 30 \\
\hline 36 & 40 & 53 & 13 & 60 \\
\hline 37 & 67 & 73 & 6 & 33 \\
\hline 38 & 70 & 90 & 20 & 30 \\
\hline 39 & 77 & 87 & 10 & 23 \\
\hline 40 & 53 & 73 & 20 & 47 \\
\hline 41 & 77 & 87 & 10 & 23 \\
\hline 42 & 67 & 73 & 6 & 33 \\
\hline 43 & 83 & 97 & 14 & 17 \\
\hline 44 & 67 & 73 & 6 & 33 \\
\hline 45 & 77 & 83 & 6 & 23 \\
\hline 46 & 73 & 73 & 0 & 27 \\
\hline 47 & 67 & 80 & 13 & 33 \\
\hline 48 & 67 & 87 & 20 & 33 \\
\hline 49 & 87 & 97 & 10 & 13 \\
\hline 50 & 67 & 73 & 6 & 33 \\
\hline Average & 71.2 & 82.2 & 11 & 28.8 \\
\hline
\end{tabular}

Table 7.6: Possible and actual gain-in-prox for FDs 


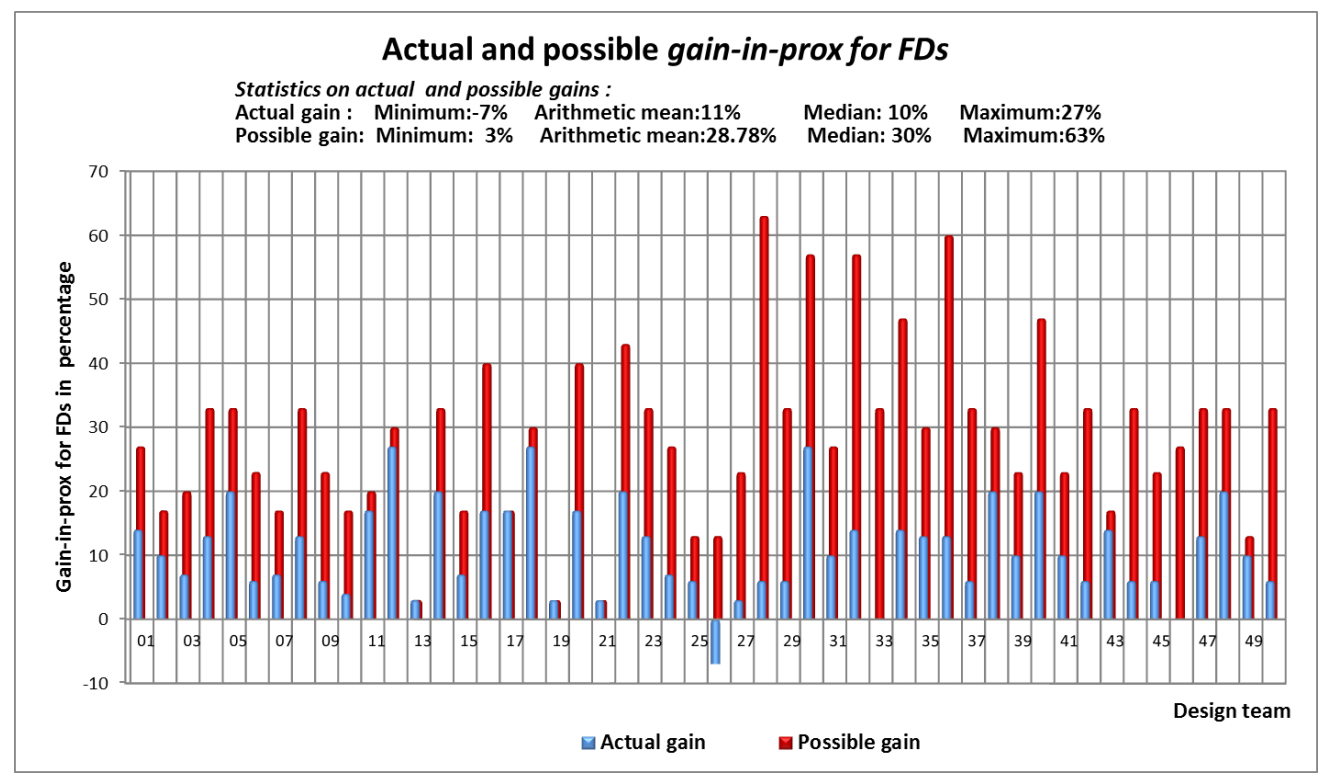

Figure 7.6: Proximity measures for FDs in percent

\section{Proximity for FDs}

Table 7.6 shows the results for the proximity measures as they apply to functional dependencies for each of the 50 design teams that participated in the experiments. The first two columns show the proximity that each team scored in phase 1 (that is, before inspecting Armstrong tables) and phase 2 (that is, after inspecting Armstrong tables) with respect to the target set. The next column displays the actual gains in proximity achieved by the teams. The last column shows the possible gain as possible improvement each team could have potentially achieved with the help of Armstrong tables. Along with Table 7.6, Figure 7.6 visualizes differences between possible and actual gains in proximity for functional dependencies for each of the teams, and shows basic statistics.

The main observation from Table 7.6 is that Armstrong tables should be considered useful in terms of proximity for functional dependencies. 
That is, it is likely that, by the inspection of Armstrong tables, teams will improve the overall quality of the functional dependencies. Indeed, our quantitative data analysis shows a significant $11 \%$ average gain (i.e. arithmetic mean) in proximity for functional dependencies. We further observe that most of the teams obtain higher proximity measures in phase 2. Only team 26 incurs a loss (7\%), teams 33 and 46 maintain their score, and the rest of the teams achieve a gain ranging from $3 \%$ to $27 \%$. The maximal gain is $27 \%$ achieved by three teams (namely, teams 12,18 and 30 ). The minimal gain of $3 \%$ is recorded for four teams (namely, teams 13, 19, 21, and 27).

Figure 7.6 illustrates the considerable impact of Armstrong tables on the proximity of functional dependencies even better. It is easy to observe that, on average, the actual gains are more than one third of the possible gains in proximity for functional dependencies. In fact, possible gains average around $28.8 \%$ and actual gains average around $11 \%$. This behavior is consistent with that of soundness and completeness: soundness has almost no actual gains while actual and possible gains are nearly the same for completeness.

In summary, we can extend our previous observation on the proximity from uniqueness constraints to functional dependencies. That is, by inspecting Armstrong tables it is likely for database designers to improve the overall quality of the functional dependencies that they acquire.

\subsubsection{Quantitative analysis for groups}

The final subsection of the quantitative analysis compares the measures between the four different groups of students that took part in the experiments. These include the computer science students from the University of Auckland (CS AU), the computer science students from the Victoria University of Wellington (CS VUW), the information systems students from the Victoria University of Wellington (IS VUW), and the computer science 


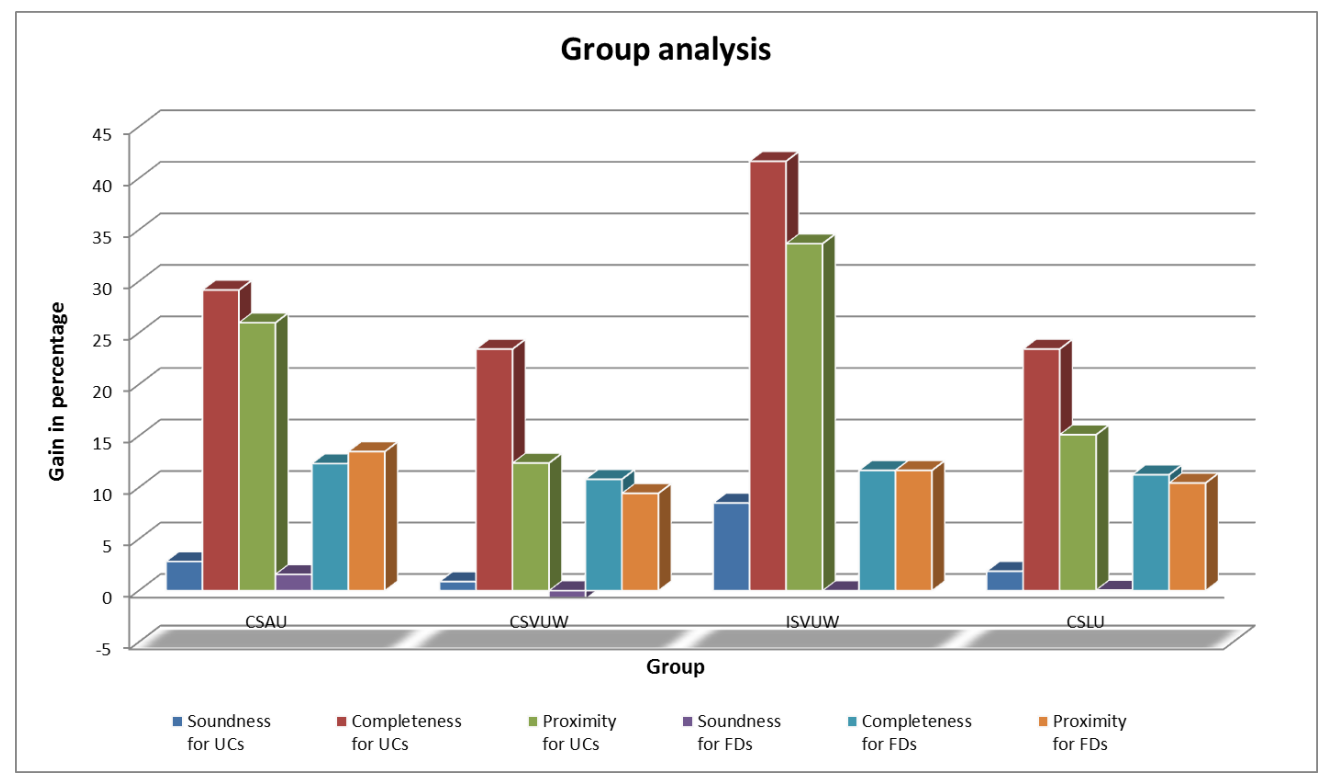

Figure 7.7: Quantitative analysis for the four different groups

students from the Lotus University (CS LU) in Vietnam. These four groups have different backgrounds and cultures, so it is interesting to investigate whether Armstrong tables have different impacts on those groups.

Figure 7.7 summarizes the different measures for all measures considered. Overall, the figure shows that: (i) IS VUW tops the lists for soundness, completeness, and proximity measured for uniqueness constraints; CS AU is the runner up, and the other groups (CS VUW and CS LU) have similar achievements. (ii) All four groups have similar soundness, completeness, and proximity measured for functional dependencies.

The data in Figure 7.7 comes from four tables: Table 7.7, Table 7.8, Table 7.9, and Table 7.10; each of which shows the corresponding gains in soundness, completeness, and proximity for the four groups.

The scores for the IS VUW teams are noticeably higher than all the others for the measures quantified for uniqueness constraints. These scores 


\begin{tabular}{|c|c|c|c|c|c|c|}
\hline \multirow{2}{*}{ Team } & \multicolumn{7}{|c|}{ gain-in- } & $\begin{array}{c}\text { sound } \\
\text { in percent }\end{array}$ & $\begin{array}{c}\text { complete }_{i}^{u} \\
\text { in percent }\end{array}$ & $\begin{array}{c}\text { prox } \\
\text { in percent }\end{array}$ & $\begin{array}{c}\text { sound }_{i}^{f} \\
\text { in percent }\end{array}$ & $\begin{array}{c}\text { complete }_{i}^{f} \\
\text { in percent }\end{array}$ & $\begin{array}{c}\text { prox }_{i}^{f} \\
\text { in percent }^{\prime}\end{array}$ \\
\hline CSAU01 & 0 & 0 & 0 & 0 & 3 & 4 \\
\hline CSAU02 & 0 & 50 & 50 & 0 & 17 & 17 \\
\hline CSAU03 & 50 & -50 & -33 & 13 & 14 & 27 \\
\hline CSAU04 & 0 & 0 & 0 & 0 & 3 & 3 \\
\hline CSAU05 & -33 & 100 & 67 & 0 & 27 & 20 \\
\hline CSAU06 & -20 & 100 & 80 & -7 & 14 & 7 \\
\hline CSAU07 & 17 & 50 & 28 & 0 & 17 & 17 \\
\hline CSAU08 & 20 & 0 & 20 & 13 & 3 & 17 \\
\hline CSAU09 & 0 & 100 & 100 & 0 & 27 & 27 \\
\hline CSAU10 & 0 & 0 & 0 & 0 & 3 & 3 \\
\hline CSAU11 & 0 & 0 & 0 & 0 & 17 & 17 \\
\hline CSAU12 & 0 & 0 & 0 & 0 & 3 & 3 \\
\hline Average & $\mathbf{2 . 8 3}$ & $\mathbf{2 9 . 2}$ & $\mathbf{2 6}$ & $\mathbf{1 . 6}$ & $\mathbf{1 2 . 3}$ & $\mathbf{1 3 . 5}$ \\
\hline
\end{tabular}

Table 7.7: Gains of CS AU teams with respect to the target set $\Sigma^{t}$

\begin{tabular}{|c|c|c|c|c|c|c|}
\hline \multirow{2}{*}{ Team } & \multicolumn{7}{|c|}{ gain-in- } & $\begin{array}{c}\text { sound } \\
\text { in percent }\end{array}$ & $\begin{array}{c}\text { complete }_{i}^{u} \\
\text { in percent }\end{array}$ & $\begin{array}{c}\text { prox }_{i}^{u} \\
\text { in percent }\end{array}$ & $\begin{array}{c}\text { sound }_{i}^{f} \\
\text { in percent }\end{array}$ & $\begin{array}{c}\text { complete }_{i}^{f} \\
\text { in percent }^{f}\end{array}$ & $\begin{array}{c}\text { prox }_{i}^{f} \\
\text { in percent }\end{array}$ \\
\hline CSVUW01 & 0 & 50 & 27 & 0 & 27 & 20 \\
\hline CSVUW02 & 17 & 50 & 34 & 0 & 13 & 13 \\
\hline CSVUW03 & -33 & 50 & 17 & -13 & 20 & 7 \\
\hline CSVUW04 & 0 & 0 & 0 & 0 & 6 & 6 \\
\hline CSVUW05 & 0 & 0 & 0 & -7 & 0 & -7 \\
\hline CSVUW06 & 0 & 0 & 0 & 0 & 3 & 3 \\
\hline CSVUW07 & 0 & 0 & 0 & 0 & 7 & 6 \\
\hline CSVUW08 & 0 & 0 & 0 & 0 & 7 & 6 \\
\hline CSVUW09 & 0 & 25 & 13 & 7 & 20 & 27 \\
\hline CSVUW10 & 0 & 0 & 0 & 0 & 10 & 10 \\
\hline CSVUW11 & 15 & 50 & 20 & 0 & 13 & 14 \\
\hline CSVUW12 & 0 & 50 & 17 & -13 & 14 & 0 \\
\hline CSVUW13 & 0 & 0 & 0 & 13 & 0 & 14 \\
\hline CSVUW14 & 0 & 50 & 50 & 0 & 13 & 13 \\
\hline CSVUW15 & 15 & 50 & 20 & 0 & 13 & 13 \\
\hline CSVUW16 & 0 & 0 & 0 & 0 & 7 & 6 \\
\hline Average & $\mathbf{0 . 9}$ & $\mathbf{2 3 . 4}$ & $\mathbf{1 2 . 4}$ & $\mathbf{- 0 . 8}$ & $\mathbf{1 0 . 8}$ & $\mathbf{9 . 4}$ \\
\hline
\end{tabular}

Table 7.8: Gains of the CS VUW teams with respect to the target set $\Sigma^{t}$ 


\begin{tabular}{|c|c|c|c|c|c|c|}
\hline \multirow{2}{*}{ Team } & \multicolumn{7}{|c|}{ gain-in- } & $\begin{array}{c}\text { sound }_{i}^{u} \\
\text { in percent }\end{array}$ & $\begin{array}{c}\text { complete }_{i}^{u} \\
\text { in percent }\end{array}$ & $\begin{array}{c}\text { prox }_{i}^{u} \\
\text { in percent }\end{array}$ & $\begin{array}{c}\text { sound }_{i}^{f} \\
\text { in percent }\end{array}$ & $\begin{array}{c}\text { complete }_{i}^{f} \\
\text { in percent }\end{array}$ & $\begin{array}{c}\text { prox }_{i}^{f} \\
\text { in percent }\end{array}$ \\
\hline ISVUW01 & 17 & 50 & 34 & 0 & 13 & 14 \\
\hline ISVUW02 & 0 & 100 & 100 & 0 & 10 & 10 \\
\hline ISVUW03 & 0 & 0 & 0 & 0 & 7 & 7 \\
\hline ISVUW04 & 17 & 50 & 34 & 0 & 13 & 13 \\
\hline ISVUW05 & 17 & 50 & 34 & 0 & 20 & 20 \\
\hline ISVUW06 & 0 & 0 & 0 & 0 & 7 & 6 \\
\hline Average & $\mathbf{8 . 5}$ & $\mathbf{4 1 . 7}$ & $\mathbf{3 3 . 7}$ & $\mathbf{0}$ & $\mathbf{1 1 . 7}$ & $\mathbf{1 1 . 7}$ \\
\hline
\end{tabular}

Table 7.9: Gains of the IS VUW teams with respect to the target set $\Sigma^{t}$

\begin{tabular}{|c|c|c|c|c|c|c|}
\hline \multirow[b]{2}{*}{ Team } & \multicolumn{6}{|c|}{ gain-in- } \\
\hline & $\begin{array}{c}\text { sound }_{i}^{u} \\
\text { in percent }\end{array}$ & $\begin{array}{l}\text { complete }_{i}^{u} \\
\text { in percent }\end{array}$ & $\begin{array}{c}\text { prox }_{i}^{u} \\
\text { in percent }\end{array}$ & $\begin{array}{c}\text { sound }_{i}^{f} \\
\text { in percent }\end{array}$ & $\begin{array}{l}\text { complete }_{i}^{f} \\
\text { in percent }\end{array}$ & $\begin{array}{c}\text { prox }_{i}^{f} \\
\text { in percent }\end{array}$ \\
\hline CSLU01 & 0 & 0 & 0 & 0 & 6 & 7 \\
\hline CSLU02 & 17 & 50 & 34 & 0 & 13 & 13 \\
\hline CSLU03 & 0 & 0 & 0 & 0 & 7 & 6 \\
\hline CSLU04 & 50 & 0 & 17 & 14 & 6 & 20 \\
\hline CSLU05 & 0 & 0 & 0 & 0 & 10 & 10 \\
\hline CSLU06 & 25 & 75 & 36 & 0 & 27 & 20 \\
\hline CSLU07 & -13 & 0 & -13 & 0 & 13 & 10 \\
\hline CSLU08 & 0 & 0 & 0 & 0 & 7 & 6 \\
\hline CSLU09 & 0 & 0 & 0 & 0 & 14 & 14 \\
\hline CSLU10 & 0 & 0 & 0 & 0 & 7 & 6 \\
\hline CSLU11 & 0 & 0 & 0 & 0 & 7 & 6 \\
\hline CSLU12 & -33 & 100 & 67 & -13 & 13 & 0 \\
\hline CSLU13 & 17 & 50 & 34 & 0 & 13 & 13 \\
\hline CSLU14 & -33 & 100 & 67 & 0 & 20 & 20 \\
\hline CSLU15 & 0 & 0 & 0 & 0 & 10 & 10 \\
\hline CSLU16 & 0 & 0 & 0 & 0 & 7 & 6 \\
\hline Average & 1.9 & 23.4 & 15.1 & 0.1 & 11.3 & 10.4 \\
\hline
\end{tabular}

Table 7.10: Gains of the CS LU teams with respect to the target set $\Sigma^{t}$ 
also show that the CS AU teams are the runner up, and there are no remarkable differences between the scores for the other groups (namely CS VUW and CS LU). Regarding the soundness, for example, IS VUW show a gain of $8.5 \%$ versus gains of $2.8 \%, 0.9 \%$, and $1.9 \%$, for CS AU, CS VUW, and CS LU, respectively. The difference in scores from IS VUW is more pronounced in completeness. For example, IS VUW has a $41.7 \%$ gain-incomplete, which is significantly higher than $29.2 \%, 23.4 \%$, and $23.4 \%$, respectively. Similarly, IS VUW achieves a $33.7 \%$ gain-in-prox versus gains of $26 \%, 12.4 \%$, and $15.1 \%$.

There are no remarkable differences among the scores measured for functional dependencies in all four groups CS AU, CS VUW, IS VUW, and CS LU. For example, the gain-in-sound of the four groups are $1.6 \%,-0.8 \%$, $0 \%$, and $0.1 \%$, respectively; the gain-in-complete are $12.3 \%, 10.8 \%, 11.7 \%$, and $11.3 \%$, respectively; the gain-in-proximity are $13.5 \%, 9.4 \%, 11.7 \%$, and $10.4 \%$, respectively.

One may speculate why the information management students from Wellington gained a significantly better understanding of uniqueness constraints from inspecting Armstrong tables over the computer science students. Most likely, it is best explained by the fact that they formed the smallest group with just six teams, as opposed to the other groups which had at least twice as many teams each. Other than that, the impact of Armstrong tables appears to be invariant under the different background and cultures of students, based on the data collected.

\subsection{Qualitative data analysis}

This section analyzes the collected data from a qualitative point of view. That is, the focus of the analysis shifts from the different measures of gains towards the analysis which uniqueness constraints and which functional dependencies were removed or added after inspecting Armstrong tables. Soundness will now tell us which meaningless uniqueness constraints and 
functional dependencies were removed or added, while completeness will tell us which meaningful uniqueness constraints and functional dependencies were removed or added.

Before the qualitative analysis, we summarize the most important points of the quantitative analysis, as we aim to collect qualitative evidence for their support.

- By inspecting Armstrong tables it is unlikely for database designers to recognize meaningless uniqueness constraints or functional dependencies that they incorrectly perceive as meaningless prior to the inspection. Indeed, the satisfaction of meaningless uniqueness constraints or functional dependencies is almost impossible to be observed in Armstrong tables.

- By inspecting Armstrong tables it is likely for database designers to recognize meaningful uniqueness constraints and functional dependencies that they incorrectly perceive as meaningless prior to the inspection. Indeed, the violation of meaningful uniqueness constraints and functional dependencies is rather certain to be observed in Armstrong tables.

In favor of an effective presentation we will now utilize the first letters $D, E, T, P, R$ as abbreviations for the attribute names Date, Emp, Time, Proj, Role, respectively, of our application domain.

\subsubsection{Qualitative analysis for soundness}

Recall that soundness measures which of the constraints perceived meaningful by a design team are actually meaningful. Qualitatively speaking, we are therefore interested in which meaningless constraints were added or removed after the inspection of Armstrong tables. Adding meaningless constraints means that soundness will decrease while removing meaningless constraints means that soundness will increase. 


\section{Soundness for UCs}

Our quantitative analysis has shown that the use of Armstrong tables is ineffective for removing meaningless UCs, and unlikely to add further meaningless UCs. The following analysis continues to examine whether this is even true from a qualitative point of view.

Table 7.11 shows those actually meaningless uniqueness constraints that were explicitly removed from or explicitly added to the data of the first phase when our design teams submitted their data to us in the second phase. The first observation is that only 7 out of 50 teams made any explicit changes in terms of meaningless uniqueness constraints. However, the explicit changes from 2 of those 7 teams were not effective: team 6 removed the meaningless UC $u(E P)$ from their set in phase 1 , but it was still implied by their remaining constraints in phase 2 , and team 44 added the same meaningless UC $u(E P)$ to their set in phase 2 , but it was already implied by their constraints from phase 1 . Therefore, only 10 percent of the participating teams made explicit changes to meaningless UCs that were effective. There was only one uniqueness constraint that was discussed amongst several of those teams: two of the teams explicitly added the constraint $u(E P)$, and two other teams explicitly removed $u(E P)$.

Table 7.11 does not show the implicit changes made to meaningless uniqueness constraints in phase 2. For that purpose, we consider Table 7.12 that shows the sets of unsound UCs before (unsound $\Sigma_{\Sigma^{t}}^{u}\left(\Sigma_{1}^{i}\right)$ ) and after $\left(u n s o u n d d_{\Sigma^{t}}^{u}\left(\Sigma_{2}^{i}\right)\right)$ inspecting Armstrong tables for a team $i(i \in[1,50])$. Here,

$$
\operatorname{unsound}_{\Sigma^{t}}^{u}(\Sigma)=s(\Sigma)-s\left(\Sigma^{t}\right)
$$

captures the set of all UCs that are meaningless with respect to the target set $\Sigma^{t}$. That is, all those UC implies by $\Sigma$ that are not implied by $\Sigma^{t}$. Teams $i$, for which unsound $\Sigma_{\Sigma^{t}}^{u}\left(\Sigma_{1}^{i}\right)=\emptyset=\operatorname{unsound}_{\Sigma^{t}}^{u}\left(\Sigma_{2}^{i}\right)$, are not listed in Table 7.12 .

First of all, the table confirms that there are no effective changes in 


\begin{tabular}{lll}
\hline Team & Meaningless UCs added & Meaningless UCs removed \\
\hline 06 & - & $\mathrm{u}(\mathrm{EP})^{*}$ \\
12 & - & $\mathrm{u}(\mathrm{EP})$ \\
15 & $\mathrm{u}(\mathrm{EPR})$ & - \\
30 & $\mathrm{u}(\mathrm{EP}), \mathrm{u}(\mathrm{EPR})$ & - \\
38 & - & $\mathrm{u}(\mathrm{EP})$ \\
41 & $\mathrm{u}(\mathrm{EP})$ & - \\
44 & $\mathrm{u}(\mathrm{EP})^{*}$ & - \\
\hline
\end{tabular}

Table 7.11: Meaningless UCs added and removed after using Armstrong tables. The * superscript indicates that either the added UC was already implied, or the removed UC was still implied.

terms of meaningless UCs for teams 6 and 44, as already indicated above. Secondly, Table 7.12 shows that 37 out of 50 teams did effectively make no changes to meaningless uniqueness constraints. However, 10 teams $(14,15,22,24,30,33,40,41,46$, and 48) effectively added some meaningless uniqueness constraints in phase 2 , and only three teams $(12,17$, and 38) removed some meaningless uniqueness constraints in phase 2. Only one removal (team 17) was done implicitly, while the other two removals (teams 12 and 38) were done explicitly, see Table 7.11. On the other hand, 7 out of the 10 additions of meaningless UCs were implicit (teams 14, 22, 24, $33,40,46$, and 48), and only three explicit (teams 15, 30, and 41). Similar to the explicit changes, most of the implicit changes revolve around the uniqueness constraint $u(E P)$, with exceptions being $u(D P)$ (team 33) and $u(D P R)($ team 17$)$.

Since the removals and additions of $u(E P)$ by teams essentially cancel each other out, it can be reiterated here that the inspection of Armstrong tables does not give much opportunity to identify which of the uniqueness constraints are incorrectly perceived as meaningless. 


\begin{tabular}{|c|c|c|}
\hline Team $i$ & $\operatorname{unsound}_{\Sigma^{t}}^{u}\left(\Sigma_{1}^{i}\right)$ & $\operatorname{unsound}_{\Sigma^{t}}^{u}\left(\Sigma_{2}^{i}\right)$ \\
\hline 01 & DHP,DHPR,DP,DPR & DHP,DHPR,DP,DPR \\
\hline 04 & EHPR,EHR,EPR,ER & EHPR,EHR,EPR,ER \\
\hline 05 & EHPR,EHR,EPR,ER & EHPR,EHR,EPR,ER \\
\hline 06 & EHP,EHPR,EP,EPR & EHP,EHPR,EP,EPR \\
\hline 08 & EHPR,EHR,EPR,ER & EHPR,EHR,EPR,ER \\
\hline 09 & DHP,DHPR,DP,DPR & DHP,DHPR,DP,DPR \\
\hline 10 & DHP,DHPR,DP,DPR & DHP,DHPR,DP,DPR \\
\hline 12 & EHP,EHPR,EP,EPR & $\emptyset$ \\
\hline 14 & $\emptyset$ & EHP,EHPR,EP,EPR \\
\hline 15 & $\emptyset$ & EHPR,EPR \\
\hline 16 & DHPR,DPR,EHPR,EPR,HPR,PR & DHPR,DPR,EHPR,EPR,HPR,PR \\
\hline 17 & DHPR,DPR & $\emptyset$ \\
\hline 20 & EHPR,EHR,EPR,ER & EHPR,EHR,EPR,ER \\
\hline 22 & EHP,EHPR & EHP,EHPR,EP,EPR \\
\hline 23 & EHP,EHPR,EP,EPR & EHP,EHPR,EP,EPR \\
\hline 24 & $\emptyset$ & EHP,EHPR,EP,EPR \\
\hline 27 & DHPR,DPR & DHPR,DPR \\
\hline 29 & EHP,EHPR,EP,EPR & EHP,EHPR,EP,EPR \\
\hline 30 & DHPR,DHR & EHP,EHPR,EP,EPR \\
\hline 32 & $\begin{array}{l}\text { DHP,DHPR,DP,DPR,EHP,EHPR,EP, } \\
\text { EPR,HP,HPR,P,PR }\end{array}$ & $\begin{array}{l}\text { DHP,DHPR,DP,DPR,EHP,EHPR,EP, } \\
\text { EPR,HP,HPR,P,PR }\end{array}$ \\
\hline 33 & EHP,EHPR,EP,EPR & DHP,DHPR,DP,DPR,EHP,EHPR,EP,EPR \\
\hline 34 & E,EH,EHP,EHPR,EHR,EP,EPR,ER & E,EH,EHP,EHPR,EHR,EP,EPR,ER \\
\hline 36 & $\begin{array}{l}\text { DHP,DHPR,DP,DPR,EHP,EHPR,EP, } \\
\text { EPR,HP,HPR,P,PR }\end{array}$ & $\begin{array}{l}\text { DHP,DHPR,DP,DPR,EHP,EHPR,EP, } \\
\text { EPR,HP,HPR,P,PR }\end{array}$ \\
\hline 37 & E,EH,EHP,EHPR,EHR,EP,EPR,ER & E,EH,EHP,EHPR,EHR,EP,EPR,ER \\
\hline 38 & EHP,EHPR,EP,EPR & $\emptyset$ \\
\hline 40 & DHPR,DPR,EHPR,EPR,HPR,PR & DHPR,DPR,EHP,EHPR,EP,EPR,HPR,PR \\
\hline 41 & EHPR,EPR & EHP,EHPR,EP,EPR \\
\hline 42 & EHPR,EPR & EHPR,EPR \\
\hline 44 & E,EH,EHP,EHPR,EHR,EP,EPR,ER & E,EH,EHP,EHPR,EHR,EP,EPR,ER \\
\hline 45 & DHP,DHPR,DP,DPR & DHP,DHPR,DP,DPR \\
\hline 46 & $\emptyset$ & EHP,EHPR,EP,EPR \\
\hline 47 & EHP,EHPR,EP,EPR & EHP,EHPR,EP,EPR \\
\hline 48 & $\emptyset$ & EHP,EHPR,EP,EPR \\
\hline 50 & EHP,EHPR,EP,EPR & EHP,EHPR,EP,EPR \\
\hline
\end{tabular}

Table 7.12: Qualitative analysis of soundness for UCS 


\section{Soundness for FDs}

Our quantitative analysis has shown that the use of Armstrong tables is ineffective for removing meaningless FDs, and unlikely to add further meaningless FDs. The following analysis continues to examine whether this is even true from a qualitative point of view.

Table 7.13 shows those actually meaningless functional dependencies that were removed from or added to the data of the first phase when our design teams submitted their data to us in the second phase. The first observation is that only 9 out of 50 teams made any changes in terms of meaningless functional dependencies. Team 4 added the meaningless FD $E R \rightarrow H$, but it was already implied by their constraint set in phase 1. Therefore, only 16 percent of the participating teams made effective changes to meaningless FDs. The second observation is that, amongst the 10 meaningless FDs that were removed or added, there were 9 different ones. For the only meaningless FD that occurred twice $(E R \rightarrow P)$, one team added it (team 34) and the other team (team 30) removed it.

\begin{tabular}{lll}
\hline Team & Meaningless FDs added & Meaningless FDs removed \\
\hline 04 & $\mathrm{ER} \rightarrow \mathrm{H}^{*}$ & - \\
17 & - & $\mathrm{PR} \rightarrow \mathrm{E}$ \\
24 & $\mathrm{EP} \rightarrow \mathrm{D}$ & - \\
26 & $\mathrm{DP} \rightarrow \mathrm{R}$ & - \\
30 & - & $\mathrm{ER} \rightarrow \mathrm{P}, \mathrm{DH} \rightarrow \mathrm{E}$ \\
33 & $\mathrm{DP} \rightarrow \mathrm{E}$ & - \\
34 & $\mathrm{ER} \rightarrow \mathrm{P}$ & $\mathrm{R} \rightarrow \mathrm{P}$ \\
41 & - & $\mathrm{PR} \rightarrow \mathrm{D}$ \\
46 & $\mathrm{EP} \rightarrow \mathrm{D}$ & - \\
\hline
\end{tabular}

Table 7.13: Meaningless FDs added and removed after using Armstrong tables. The * superscript indicates that either the added FD was already implied, or the removed FD was still implied. 


\begin{tabular}{|c|c|c|}
\hline Team $i$ & unsound $_{\Sigma^{t}}^{f}\left(\Sigma_{1}^{i}\right)$ & $\operatorname{unsound}_{\Sigma^{t}}^{f}\left(\Sigma_{2}^{i}\right)$ \\
\hline 01 & DHP,DHPR,DP,DPR & DHP,DHPR,DP,DPR \\
\hline 04 & EHPR,EHR,EPR,ER & EHPR,EHR,EPR,ER \\
\hline 05 & EHPR,EHR,EPR,ER & EHPR,EHR,EPR,ER \\
\hline 06 & EHP,EHPR,EP,EPR,ER & EHP,EHPR,EP,EPR,ER \\
\hline 07 & ER & ER \\
\hline 08 & EHPR,EHR,EPR,ER & EHPR,EHR,EPR,ER \\
\hline 09 & DHP,DHPR,DP,DPR & DHP,DHPR,DP,DPR \\
\hline 10 & DHP,DHPR,DP,DPR & DHP,DHPR,DP,DPR \\
\hline 12 & EHP,EHPR,EP,EPR & $\emptyset$ \\
\hline 14 & EHP,EHPR,EP,EPR & EHP,EHPR,EP,EPR \\
\hline 15 & $\emptyset$ & EHPR,EPR \\
\hline 16 & DHPR,DPR,EHPR,EPR,HPR,PR & DHPR,DPR,EHPR,EPR,HPR,PR \\
\hline 17 & DHPR,DPR,HPR,PR & $\emptyset$ \\
\hline 20 & EHPR,EHR,EPR,ER & EHPR,EHR,EPR,ER \\
\hline 22 & EH,EHP,EHPR,EHR,EP,EPR,ER & EH,EHP,EHPR,EHR,EP,EPR,ER \\
\hline 23 & EHP,EHPR,EP,EPR & EHP,EHPR,EP,EPR \\
\hline 24 & $\emptyset$ & EHP,EHPR,EP,EPR \\
\hline 26 & $\emptyset$ & DHP,DP \\
\hline 27 & DHPR,DPR,HPR,PR & DHPR,DPR,HPR,PR \\
\hline 28 & $\begin{array}{l}\text { DHP,DHPR,DHR,DP,DPR,DR,ER, } \\
\text { HP,HPR,HR,P,PR,R }\end{array}$ & $\begin{array}{l}\text { DHP,DHPR,DHR,DP,DPR,DR,ER, } \\
\text { HP,HPR,HR,P,PR,R }\end{array}$ \\
\hline 29 & E,EH,EHP,EHPR,EHR,EP,EPR,ER & E,EH,EHP,EHPR,EHR,EP,EPR,ER \\
\hline 30 & DH,DHP,DHPR,DHR,EHR,ER & EHP,EHPR,EP,EPR \\
\hline 31 & E,EH,EHR,ER & E,EH,EHR,ER \\
\hline 32 & $\begin{array}{l}\text { DHP,DHPR,DP,DPR,EH,EHP,EHPR, } \\
\text { EP,EPR,HP,HPR,P,PR }\end{array}$ & $\begin{array}{l}\text { DHP,DHPR,DP,DPR,EH,EHP,EHPR, } \\
\text { EP,EPR,HP,HPR,P,PR }\end{array}$ \\
\hline 33 & DR,EHP,EHPR,EP,EPR & DHP,DHPR,DP,DPR,DR,EHP,EHPR,EP,EPR \\
\hline 34 & $\begin{array}{l}\text { DHR,DR,E,EH,EHP,EHPR,EHR, } \\
\text { EP,EPR,ER,HR,R }\end{array}$ & E,EH,EHP,EHPR,EHR,EP,EPR,ER \\
\hline 36 & $\begin{array}{l}\text { DHP,DHPR,DP,DPR,E,EH,EHP,EHPR, } \\
\text { EP,EPR,HP,HPR,P,PR }\end{array}$ & $\begin{array}{l}\text { DHP,DHPR,DP,DPR,E,EH,EHP,EHPR, } \\
\text { EP,EPR,HP,HPR,P,PR }\end{array}$ \\
\hline 37 & E,EH,EHP,EHPR,EHR,EP,EPR,ER & E,EH,EHP,EHPR,EHR,EP,EPR,ER \\
\hline 38 & EHP,EHPR,EP,EPR,ER & ER \\
\hline 39 & DHP,DP & DHP,DP \\
\hline 40 & DHPR,DPR,EHP,EHPR,EP,EPR,HPR,PR & DHPR,DPR,EHP,EHPR,EP,EPR,HPR,PR \\
\hline 41 & EHPR,EPR,HPR,PR & EHP,EHPR,EP,EPR \\
\hline 42 & EHPR,EPR & EHPR,EPR \\
\hline 44 & E,EH,EHP,EHPR,EHR,EP,EPR,ER & E,EH,EHP,EHPR,EHR,EP,EPR,ER \\
\hline 45 & DHP,DHPR,DP,DPR & DHP,DHPR,DP,DPR \\
\hline 46 & E,EH & E,EH,EHP,EHPR,EP,EPR \\
\hline 47 & EHP,EHPR,EP,EPR & EHP,EHPR,EP,EPR \\
\hline 48 & EHP,EHPR,EP,EPR & EHP,EHPR,EP,EPR \\
\hline 50 & EHP,EHPR,EP,EPR & EHP,EHPR,EP,EPR \\
\hline
\end{tabular}

Table 7.14: Qualitative analysis of soundness for FDs 
Table 7.13 does not show implicit changes to the meaningful functional dependencies made by the teams. For that purpose we look at Table 7.14. Here,

$$
\operatorname{unsound}_{\Sigma^{t}}^{f}(\Sigma)=\left\{X \in \mathcal{P}_{0}(R) \mid X_{\Sigma, R_{s}}^{*} \not X_{\Sigma^{t}, R_{s}}^{*}\right\}
$$

captures the set of all attribute sets that are meaningless with respect to the target set $\Sigma^{t}$. That is, all those attribute sets that functionally determine some attributes with respect to $\Sigma$ that are not functionally determined with respect to $\Sigma^{t}$. Teams $i$, for which unsound $_{\Sigma^{t}}^{f}\left(\Sigma_{1}^{i}\right)=\emptyset=$ unsound $_{\Sigma^{t}}^{f}\left(\Sigma_{2}^{i}\right)$, are not listed.

Table 7.14 shows that only 11 out of 50 teams made implicit changes. Six teams $(12,17,30,34,38,41)$ implicitly removed some meaningless FDs, and six teams $(15,24,26,33,34$, and 46$)$ implicitly added some meaningful FDs. Note that team 34 did both. Each of these implicit changes results either from an explicit change as observed in Table 7.13 (for teams 17, 24, $26,30,33,34,41$, and 46 ) or from the explicit change of a meaningless UC as observed in Table 7.11 (for teams 12, 15, and 38).

We conclude that the inspection of Armstrong tables does not give much opportunity to identify which of the functional dependencies are incorrectly perceived as meaningless.

\subsubsection{Qualitative analysis for completeness}

Our quantitative analysis has shown that the use of Armstrong tables is effective for adding meaningful UCs and FDs. The following analysis continues to examine whether this is even true from a qualitative point of view.

\section{Completeness for UCs}

Table 7.15 shows those actually meaningful functional dependencies that were added to or removed from the data of the first phase when our design 


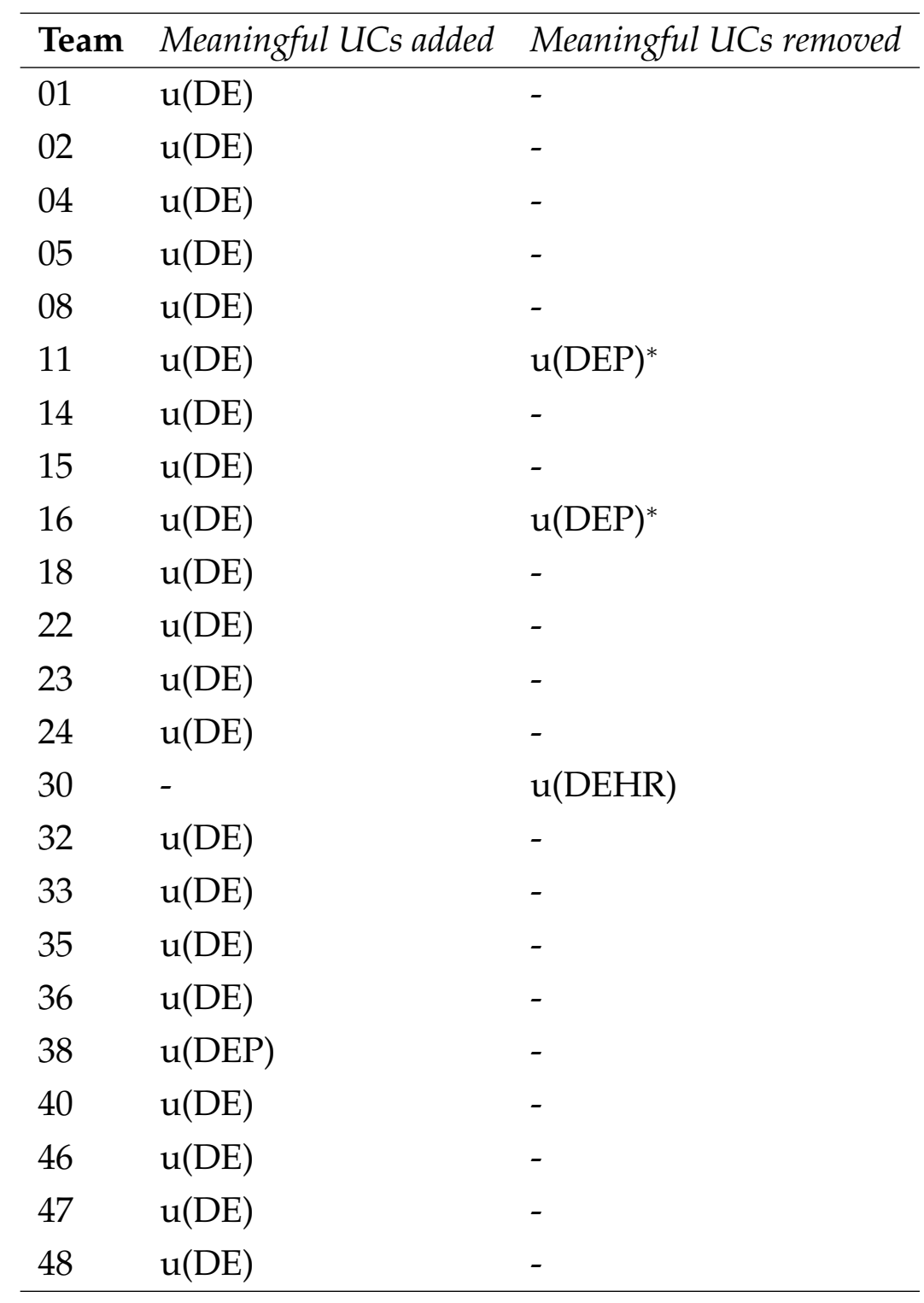

Table 7.15: Meaningful UCs added and removed after using Armstrong tables. The * superscript indicates that either the added UC was already implied, or the removed UC was still implied. 


\begin{tabular}{|c|c|c|}
\hline Team $i$ & incomplete $\Sigma^{u}\left(\Sigma_{1}^{i}\right)$ & incomplete $\Sigma_{\Sigma^{t}}^{u}\left(\Sigma_{2}^{i}\right)$ \\
\hline 01 & DE,DEH,DEHR,DER & $\emptyset$ \\
\hline 02 & DE,DEH,DEHP,DEHR,DEP,DEPR,DER,DEHPR & $\emptyset$ \\
\hline 03 & DE,DEH,DEHR,DER & DE,DEH,DEHR,DER \\
\hline 04 & DE,DEH,DEHP,DEP & $\emptyset$ \\
\hline 05 & DE,DEH,DEHP,DEP & $\emptyset$ \\
\hline 06 & $\emptyset$ & $\emptyset$ \\
\hline 07 & $\emptyset$ & $\emptyset$ \\
\hline 08 & DE,DEH,DEHP,DEP & $\emptyset$ \\
\hline 09 & $\emptyset$ & $\emptyset$ \\
\hline 10 & $\emptyset$ & $\emptyset$ \\
\hline 11 & DE,DEH,DEHR,DER & $\emptyset$ \\
\hline 12 & DE,DEH,DEHR,DER & DE,DEH,DEHP,DEHR,DEP,DEPR,DER,DEHPR \\
\hline 13 & $\emptyset$ & $\emptyset$ \\
\hline 14 & DE,DEH,DEHP,DEHR,DEP,DEPR,DER,DEHPR & $\emptyset$ \\
\hline 15 & DE,DEH,DEHP,DEHR,DEP,DEPR,DER,DEHPR & $\emptyset$ \\
\hline 16 & DE,DEH,DEHR,DER & $\emptyset$ \\
\hline 17 & $\emptyset$ & $\emptyset$ \\
\hline 18 & DE,DEH,DEHP,DEHR,DEP,DEPR,DER,DEHPR & $\emptyset$ \\
\hline 19 & $\emptyset$ & $\emptyset$ \\
\hline 20 & DE,DEH,DEHP,DEP & DE,DEH,DEHP,DEP \\
\hline 21 & $\emptyset$ & $\emptyset$ \\
\hline 22 & DE,DEP,DEPR,DER & $\emptyset$ \\
\hline 23 & DE,DEH,DEHR,DER & $\emptyset$ \\
\hline 24 & DE,DEH,DEHR,DER & $\emptyset$ \\
\hline 25 & $\emptyset$ & $\emptyset$ \\
\hline 26 & $\emptyset$ & $\emptyset$ \\
\hline 27 & $\emptyset$ & $\emptyset$ \\
\hline 28 & DE,DEH,DEHP,DEHR,DEP,DEPR,DER,DEHPR & DE,DEH,DEHP,DEHR,DEP,DEPR,DER,DEHPR \\
\hline 29 & $\emptyset$ & $\emptyset$ \\
\hline 30 & DE,DEH,DEHP,DEP,DEPR,DER & DE,DEH,DEHR,DER \\
\hline 31 & $\emptyset$ & $\emptyset$ \\
\hline 32 & DE,DEH,DEHR,DER & $\emptyset$ \\
\hline 33 & DE,DEH,DEHR,DER & $\emptyset$ \\
\hline 34 & $\emptyset$ & $\emptyset$ \\
\hline 35 & DE,DEH,DEHR,DER & $\emptyset$ \\
\hline 36 & DE,DEH,DEHR,DER & $\emptyset$ \\
\hline 37 & $\emptyset$ & $\emptyset$ \\
\hline 38 & DE,DEH,DEHR,DER & DE,DEH,DEHR,DER \\
\hline 39 & $\emptyset$ & $\emptyset$ \\
\hline 40 & DE,DEH,DEHP,DEHR,DEP,DER & $\emptyset$ \\
\hline 41 & $\emptyset$ & $\emptyset$ \\
\hline 42 & DE,DEH,DEHP,DEHR,DEP,DER & DE,DEH,DEHP,DEHR,DEP,DER \\
\hline 43 & $\emptyset$ & $\emptyset$ \\
\hline 44 & $\emptyset$ & $\emptyset$ \\
\hline 45 & $\emptyset$ & $\emptyset$ \\
\hline 46 & DE,DEH,DEHP,DEHR,DEP,DEPR,DER,DEHPR & $\emptyset$ \\
\hline 47 & DE,DEH,DEHR,DER & $\emptyset$ \\
\hline 48 & DE,DEH,DEHP,DEHR,DEP,DEPR,DER,DEHPR & $\emptyset$ \\
\hline 49 & $\emptyset$ & $\emptyset$ \\
\hline 50 & DE,DEH,DEHR,DER & DE,DEH,DEHR,DER \\
\hline
\end{tabular}

Table 7.16: Qualitative analysis of completeness for UCs 
teams submitted their data to us in the second phase. The most pressing observation is that 21 out of 50 teams $(1,2,4,5,8,11,14-16,18,22-24,32$, $33,35,36,40,46-48)$ explicitly added the meaningful $\mathrm{UC} u(D E)$ in phase 2. 21 other teams $(6,7,9,10,13,17,19,21,25-27,29,31,34,37,39,41,43-45$, and 49 ) had already included this UC in phase 1 . Team 38 added $u(E D P)$, but did not include $u(E D)$. Two teams (11 and 16) replaced the meaningful $\mathrm{UC} u(D E P)$ from phase 1 by the meaningful $\mathrm{UC} u(E D)$. Therefore, only one team (30) explicitly removed a meaningful UC, namely $u(D E H R)$.

Table 7.16 takes this analysis a step further by showing explicitly which meaningful uniqueness constraints are missing after each phase. Here,

$$
\text { incomplete } \Sigma_{\Sigma^{t}}^{u}(\Sigma)=s\left(\Sigma^{t}\right)-s(\Sigma)
$$

captures the set of all meaningful UCs that are not captured by $\Sigma$. That is, all those UC implied by $\Sigma^{t}$ but not implied by $\Sigma$.

Strikingly, 42 out of 50 teams have identified all meaningful UCs after phase 2. For 6 of the remaining 8 teams $(3,20,28,38,42$, and 50) changes occur between phase 1 and phase 2 . Team 12 explicitly removed the meaningless $\mathrm{UC} u(E P)$ in phase 2, and thereby also removed some meaningful UCs implicitly. Team 30 explicitly added the meaningless UC $u(E P)$ in phase 2 , and thereby also added some meaningful UCs implicitly.

The qualitative analysis further details how the quantitative evidence has been derived that the inspection of Armstrong tables helps database designer recognize additional UCs.

\section{Completeness for FDs}

We first analyze Table 7.17 to identify which meaningful FDs are removed and added after inspecting Armstrong tables.

A strong majority of the teams ( 40 out of 50 teams) added at least one meaningful FD in phase 2. 12 out of those 40 teams $(1,3,5,6,7,14,25,30$, $41,43,44$, and 48) added two meaningful FDs, and three teams (12, 20, and 38) added three meaningful FDs. The most popular FD is Proj,Role $\rightarrow$ Hrs 


\begin{tabular}{|c|c|c|}
\hline Team & Meaningful FDs added & Meaningful FDs removed \\
\hline 01 & $\mathrm{EP} \rightarrow \mathrm{H}, \mathrm{EP} \rightarrow \mathrm{R}$ & $\mathrm{DE} \rightarrow \mathrm{P}^{*}, \mathrm{DE} \rightarrow \mathrm{R}^{*}$ \\
\hline 03 & $\mathrm{EP} \rightarrow \mathrm{H}, \mathrm{DE} \rightarrow \mathrm{P}$ & - \\
\hline 04 & $\mathrm{EP} \rightarrow \mathrm{H}$ & - \\
\hline 05 & $\mathrm{EP} \rightarrow \mathrm{R}, \mathrm{PR} \rightarrow \mathrm{H}$ & - \\
\hline 06 & $\mathrm{EP} \rightarrow \mathrm{R}, \mathrm{PR} \rightarrow \mathrm{H}$ & - \\
\hline 07 & $\mathrm{EP} \rightarrow \mathrm{H}, \mathrm{EP} \rightarrow \mathrm{R}$ & $\mathrm{DEP} \rightarrow \mathrm{H}^{*}$ \\
\hline 08 & $\mathrm{EP} \rightarrow \mathrm{H}$ & $\mathrm{DE} \rightarrow \mathrm{P}^{*}$ \\
\hline 09 & $\mathrm{EP} \rightarrow \mathrm{R}$ & - \\
\hline 10 & $\mathrm{PR} \rightarrow \mathrm{H}$ & - \\
\hline 11 & $\mathrm{EP} \rightarrow \mathrm{R}$ & - \\
\hline 12 & $\mathrm{DE} \rightarrow \mathrm{R}, \mathrm{DE} \rightarrow \mathrm{H}, \mathrm{PR} \rightarrow \mathrm{H}$ & $\mathrm{EPR} \rightarrow \mathrm{H}^{*}$ \\
\hline 13 & $\mathrm{EP} \rightarrow \mathrm{H}$ & - \\
\hline 14 & $\mathrm{EP} \rightarrow \mathrm{H}^{*}, \mathrm{EP} \rightarrow \mathrm{R}^{*}$ & - \\
\hline 16 & $\mathrm{EP} \rightarrow \mathrm{R}$ & - \\
\hline 17 & $\mathrm{EP} \rightarrow \mathrm{H}$ & - \\
\hline 18 & $\mathrm{PR} \rightarrow \mathrm{H}$ & $\mathrm{DE} \rightarrow \mathrm{P}^{*}$ \\
\hline 19 & $\mathrm{EP} \rightarrow \mathrm{H}$ & - \\
\hline 20 & $\mathrm{DE} \rightarrow \mathrm{R}^{*}, \mathrm{EP} \rightarrow \mathrm{R}, \mathrm{PR} \rightarrow \mathrm{H}$ & - \\
\hline 21 & $\mathrm{EP} \rightarrow \mathrm{H}$ & - \\
\hline 22 & $\mathrm{PR} \rightarrow \mathrm{H}$ & - \\
\hline 23 & $\mathrm{EP} \rightarrow \mathrm{H}$ & - \\
\hline 25 & $\mathrm{DE} \rightarrow \mathrm{R}^{*}, \mathrm{EP} \rightarrow \mathrm{H}$ & - \\
\hline 27 & $\mathrm{EP} \rightarrow \mathrm{R}$ & - \\
\hline 28 & $\mathrm{EP} \rightarrow \mathrm{R}$ & - \\
\hline 29 & $\mathrm{PR} \rightarrow \mathrm{H}$ & - \\
\hline 30 & $\mathrm{DEH} \rightarrow \mathrm{P}, \mathrm{EP} \rightarrow \mathrm{R}^{*}$ & - \\
\hline 31 & $\mathrm{PR} \rightarrow \mathrm{H}$ & - \\
\hline 32 & $\mathrm{EP} \rightarrow \mathrm{H}^{*}$ & - \\
\hline 37 & $\mathrm{PR} \rightarrow \mathrm{H}$ & - \\
\hline 38 & $\mathrm{DE} \rightarrow \mathrm{R}, \mathrm{EP} \rightarrow \mathrm{R}, \mathrm{PR} \rightarrow \mathrm{H}$ & - \\
\hline 39 & $\mathrm{PR} \rightarrow \mathrm{H}$ & - \\
\hline 40 & $\mathrm{EP} \rightarrow \mathrm{R}$ & - \\
\hline 41 & $\mathrm{PR} \rightarrow \mathrm{H}, \mathrm{DE} \rightarrow \mathrm{R}^{*}$ & - \\
\hline 42 & $\mathrm{DE} \rightarrow \mathrm{P}$ & - \\
\hline 43 & $\mathrm{EP} \rightarrow \mathrm{R}, \mathrm{PR} \rightarrow \mathrm{H}$ & $\mathrm{DE} \rightarrow \mathrm{R}^{*}$ \\
\hline 44 & $\mathrm{EP} \rightarrow \mathrm{R}^{*}, \mathrm{PR} \rightarrow \mathrm{H}$ & $\mathrm{EP} \rightarrow \mathrm{H}^{*}$ \\
\hline 45 & $\mathrm{PR} \rightarrow \mathrm{H}$ & - \\
\hline 48 & $\mathrm{PR} \rightarrow \mathrm{H}, \mathrm{EP} \rightarrow \mathrm{R}^{*}$ & - \\
\hline 49 & $\mathrm{PR} \rightarrow \mathrm{H}$ & - \\
\hline 50 & $\mathrm{PR} \rightarrow \mathrm{H}$ & - \\
\hline
\end{tabular}

Table 7.17: Meaningful FDs added and removed after using Armstrong tables. The * superscript indicates that either the added FD was already implied, or the removed FD was still implied. 


\begin{tabular}{|c|c|c|}
\hline Team $i$ & incomplete $_{\Sigma^{t}}^{f}\left(\Sigma_{1}^{i}\right)$ & incomplete $_{\Sigma^{t}}^{f}\left(\Sigma_{2}^{i}\right)$ \\
\hline 01 & DE,DER,EHP,EP & $\emptyset$ \\
\hline 02 & DE,DEH,DER,DPR,PR & DPR,PR \\
\hline 03 & DE,DEH,DEHR,DER,EHP,EP & DE,DER,EHP,EP \\
\hline 04 & DE,DEH,DEP,DPR,EP,PR & DPR,PR \\
\hline 05 & DE,DEH,DPR,EHP,EP,PR & $\emptyset$ \\
\hline 06 & DPR,PR & $\emptyset$ \\
\hline 07 & DPR,EHP,EP,PR & DPR,PR \\
\hline 08 & DE,DEH,DEP,DPR,EP,PR & DPR,PR \\
\hline 09 & EP,EPR,PR & PR \\
\hline 10 & PR & $\emptyset$ \\
\hline 11 & DE,DEH,DEHR,DER,EHP,EP & EP \\
\hline 12 & DE,DEH,DER,DPR,PR & EP \\
\hline 13 & EP & $\emptyset$ \\
\hline 14 & DE,DEH,DEHP,DEHR,DEP,DER,EHP,EP & $\emptyset$ \\
\hline 15 & DE,DEH,DEP,DER,EP & EP \\
\hline 16 & DE,DEH,DEHR,DER,EHP,EP & EP \\
\hline 17 & EP & $\emptyset$ \\
\hline 18 & DE,DEH,DEP,DEPR,DER,DPR,EP,EPR,PR & EP \\
\hline 19 & EP & $\emptyset$ \\
\hline 20 & DE,DEH,DEHP,DEP,DPR,EHP,EP,PR & DE,DEP,EP \\
\hline 21 & EP & $\emptyset$ \\
\hline 22 & DE,DEP,DEPR,DER,DPR,EP,EPR,PR & $\emptyset$ \\
\hline 23 & DE,DEH,DEHR,DER,DPR,PR & DPR,PR \\
\hline 24 & DE,DEH,DEHR,DER,DPR,EP,EPR,PR & DPR,PR \\
\hline 25 & DPR,EP,EPR,PR & DPR,PR \\
\hline 26 & DPR,EP,EPR,PR & DPR,EP,EPR,PR \\
\hline 27 & EHP,EP,EPR,PR & EP,EPR,PR \\
\hline 28 & DE,DEH,DEHP,DEP,EHP,EP & DE,DEH,DEP,EP \\
\hline 29 & DPR,PR & $\emptyset$ \\
\hline 30 & DE,DEH,DEHP,DEP,DEPR,DER,DPR,EHP,EP,EPR,PR & DE,DEH,DER,DPR,PR \\
\hline 31 & DPR,EP,EPR,PR & $\mathrm{EP}$ \\
\hline 32 & DE,DEH,DEHR,DER & $\emptyset$ \\
\hline 33 & DE,DEH,DEHR,DER,PR & PR \\
\hline 34 & DPR,PR & DPR,PR \\
\hline 35 & DE,DEH,DEHR,DER,DPR,EHP,EP,EPR,PR & DPR,EHP,EP,EPR,PR \\
\hline 36 & DE,DEH,DEHR,DER & $\emptyset$ \\
\hline 37 & DPR,PR & $\emptyset$ \\
\hline 38 & DE,DEH,DPR,PR & $\mathrm{DE}, \mathrm{EP}$ \\
\hline 39 & DPR,EHP,EP,EPR,PR & EHP,EP \\
\hline 40 & DE,DEH,DEHP,DEHR,DEP,DER,EHP,EP & $\emptyset$ \\
\hline 41 & DPR,EHP,EP,PR & $\emptyset$ \\
\hline 42 & DE,DEH,DEHR,DER,DPR,EHP,EP,PR & DE,DER,DPR,EHP,EP,PR \\
\hline 43 & DPR,EHP,EP,EPR,PR & $\mathrm{EP}$ \\
\hline 44 & DPR,PR & $\emptyset$ \\
\hline 45 & EP,EPR,PR & $\mathrm{EP}$ \\
\hline 46 & DE,DEH,DEHR,DER,DPR,PR & DPR,PR \\
\hline 47 & DE,DEH,DEHR,DER,DPR,PR & DPR,PR \\
\hline 48 & DE,DEH,DEHR,DER,DPR,PR & $\emptyset$ \\
\hline 49 & DPR,EP,EPR,PR & EP \\
\hline 50 & DE,DEH,DEHR,DER,DPR,PR & DE,DEH,DEHR,DER \\
\hline
\end{tabular}

Table 7.18: Qualitative analysis of completeness for FDs 
which was added by 19 teams $(5,6,10,12,18,20,22,29,31,37-39,41,43-$ $45,48-50)$ in phase 2. The runner-up is Emp,Proj $\rightarrow$ Role added by 17 teams $(1,5-7,9,11,14,16,20,27,28,30,38,40,43,44$, and 48). Emp,Proj $\rightarrow H r s$ is in third place, added by 13 teams $(1,3,4,7,8,13,14,17,19,21,23,25$, and 32). An important observation is that all of the meaningful FDs that were removed in phase 2 are still implied by the corresponding constraint sets. Hence, there was no effective loss in completeness due to the removal of any meaningful FDs.

In analogy with the completeness analysis for UCs, Table 7.18 shows a remarkable increase in the number of recognized meaningful FDs when moving from phase 1 to 2 . Here,

$$
\text { incomplete }_{\Sigma^{t}}^{f}(\Sigma)=\left\{X \in \mathcal{P}_{0}(R) \mid X_{\Sigma^{t}, R_{s}}^{*} \nsubseteq X_{\Sigma, R_{s}}^{*}\right\}
$$

captures the set of all attribute sets that are meaningful with respect to the target set $\Sigma^{t}$ but not captured by $\Sigma$. That is, all those attribute sets that functionally determine some attributes with respect to $\Sigma^{t}$ that are not functionally determined with respect to $\Sigma$.

While none of the teams recognized all meaningful FDs after phase 1, there are 18 teams that did recognize all meaningful FDs after phase 2 (1, $5,6,10,13,14,17,19,21,22,29,32,36,37,40,41,44$, and 48). As indicated above, there are no remarkable differences between the numbers of times that teams recognized a meaningful FD in phase 2. The same is true for the numbers of times that a meaningful FD was not recognized after phase 2.

We conclude that the inspection of Armstrong tables helps database designer recognize additional FDs.

\subsubsection{Qualitative analysis for proximity}

Recall that the proximity between two sets of UCs and FDs measures their degree of equivalence. Proximity combines soundness and completeness, 


\begin{tabular}{|c|c|c|}
\hline Team $i$ & $\operatorname{dist}^{u}\left(\Sigma_{1}^{i}, \Sigma^{t}\right)$ & $\operatorname{dist}^{u}\left(\Sigma_{2}^{i}, \Sigma^{t}\right)$ \\
\hline 01 & $\begin{array}{l}\text { DHP,DHPR,DP,DPR,DE,DEH,DEHR,DER } \\
\end{array}$ & DHP,DHPR,DP,DPR \\
\hline 02 & DE,DEH,DEHP,DEHR,DEP,DEPR,DER,DEHPR & $\emptyset$ \\
\hline 03 & DE,DEH,DEHR,DER & DE,DEH,DEHR,DER \\
\hline 04 & EHPR,EHR,EPR,ER,DE,DEH,DEHP,DEP & EHPR,EHR,EPR,ER \\
\hline 05 & EHPR,EHR,EPR,ER,DE,DEH,DEHP,DEP & EHPR,EHR,EPR,ER \\
\hline 06 & EHP,EHPR,EP,EPR & EHP,EHPR,EP,EPR \\
\hline 07 & $\emptyset$ & $\emptyset$ \\
\hline 08 & EHPR,EHR,EPR,ER,DE,DEH,DEHP,DEP & EHPR,EHR,EPR,ER \\
\hline 09 & DHP,DHPR,DP,DPR & DHP,DHPR,DP,DPR \\
\hline 10 & DHP,DHPR,DP,DPR & DHP,DHPR,DP,DPR \\
\hline 11 & DE,DEH,DEHR,DER & $\emptyset$ \\
\hline 12 & EHP,EHPR,EP,EPR,DE,DEH,DEHR,DER & DE,DEH,DEHP,DEHR,DEP,DEPR,DER,DEHPR \\
\hline 13 & $\emptyset$ & $\emptyset$ \\
\hline 14 & DE,DEH,DEHP,DEHR,DEP,DEPR,DER,DEHPR & EHP,EHPR,EP,EPR \\
\hline 15 & DE,DEH,DEHP,DEHR,DEP,DEPR,DER,DEHPR & EHPR,EPR \\
\hline 16 & DHPR,DPR,EHPR,EPR,HPR,PR,DE,DEH,DEHR,DER & DHPR,DPR,EHPR,EPR,HPR,PR \\
\hline 17 & DHPR,DPR & $\emptyset$ \\
\hline 18 & DE,DEH,DEHP,DEHR,DEP,DEPR,DER,DEHPR & $\emptyset$ \\
\hline 19 & $\emptyset$ & $\emptyset$ \\
\hline 20 & EHPR,EHR,EPR,ER,DE,DEH,DEHP,DEP & EHPR,EHR,EPR,ER,DE,DEH,DEHP,DEP \\
\hline 21 & $\emptyset$ & $\emptyset$ \\
\hline 22 & EHP,EHPR,DE,DEP,DEPR,DER & EHP,EHPR,EP,EPR \\
\hline 23 & EHP,EHPR,EP,EPR,DE,DEH,DEHR,DER & EHP,EHPR,EP,EPR \\
\hline 24 & DE,DEH,DEHR,DER & EHP,EHPR,EP,EPR \\
\hline 25 & $\emptyset$ & $\emptyset$ \\
\hline 26 & $\emptyset$ & $\emptyset$ \\
\hline 27 & DHPR,DPR & DHPR,DPR \\
\hline 28 & DE,DEH,DEHP,DEHR,DEP,DEPR,DER,DEHPR & DE,DEH,DEHP,DEHR,DEP,DEPR,DER,DEHPR \\
\hline 29 & EHP,EHPR,EP,EPR & EHP,EHPR,EP,EPR \\
\hline 30 & DHPR,DHR,DE,DEH,DEHP,DEP,DEPR,DER & EHP,EHPR,EP,EPR,DE,DEH,DEHR,DER \\
\hline 31 & $\emptyset$ & $\emptyset$ \\
\hline \multirow[t]{2}{*}{32} & DHP,DHPR,DP,DPR,EHP,EHPR,EP,EPR,HP,HPR,P,PR, & DHP,DHPR,DP,DPR,EHP,EHPR,EP,EPR,HP,HPR,P,PR \\
\hline & DE,DEH,DEHR,DER & \\
\hline 33 & EHP,EHPR,EP,EPR,DE,DEH,DEHR,DER & DHP,DHPR,DP,DPR,EHP,EHPR,EP,EPR \\
\hline 34 & E,EH,EHP,EHPR,EHR,EP,EPR,ER & E,EH,EHP,EHPR,EHR,EP,EPR,ER \\
\hline 35 & DE,DEH,DEHR,DER & $\emptyset$ \\
\hline 36 & $\begin{array}{l}\text { DHP,DHPR,DP,DPR,EHP,EHPR,EP,EPR,HP,HPR,P,PR, } \\
\text { DE,DEH,DEHR,DER }\end{array}$ & DHP,DHPR,DP,DPR,EHP,EHPR,EP,EPR,HP,HPR,P,PR \\
\hline 37 & E,EH,EHP,EHPR,EHR,EP,EPR,ER & E,EH,EHP,EHPR,EHR,EP,EPR,ER \\
\hline 38 & EHP,EHPR,EP,EPR,DE,DEH,DEHR,DER & DE,DEH,DEHR,DER \\
\hline 39 & $\emptyset$ & $\emptyset$ \\
\hline \multirow[t]{2}{*}{40} & DHPR,DPR,EHPR,EPR,HPR,PR,DE, & DHPR,DPR,EHP,EHPR,EP,EPR,HPR,PR \\
\hline & DEH,DEHP,DEHR,DEP,DER & \\
\hline 41 & EHPR,EPR & EHP,EHPR,EP,EPR \\
\hline 42 & EHPR,EPR,DE,DEH,DEHP,DEHR,DEP,DER & EHPR,EPR,DE,DEH,DEHP,DEHR,DEP,DER \\
\hline 43 & $\emptyset$ & $\emptyset$ \\
\hline 44 & E,EH,EHP,EHPR,EHR,EP,EPR,ER & E,EH,EHP,EHPR,EHR,EP,EPR,ER \\
\hline 45 & DHP,DHPR,DP,DPR & DHP,DHPR,DP,DPR \\
\hline 46 & DE,DEH,DEHP,DEHR,DEP,DEPR,DER,DEHPR & EHP,EHPR,EP,EPR \\
\hline 47 & EHP,EHPR,EP,EPR,DE,DEH,DEHR,DER & EHP,EHPR,EP,EPR \\
\hline 48 & DE,DEH,DEHP,DEHR,DEP,DEPR,DER,DEHPR & EHP,EHPR,EP,EPR \\
\hline 49 & $\emptyset$ & $\emptyset$ \\
\hline 50 & EHP,EHPR,EP,EPR,DE,DEH,DEHR,DER & $\begin{array}{l}\text { EHP,EHPR,EP,EPR,DE,DEH,DEHR,DER } \\
\end{array}$ \\
\hline
\end{tabular}

Table 7.19: Qualitative analysis of proximity for UCs 


\begin{tabular}{|c|c|c|}
\hline Team $i$ & $\operatorname{dist}^{f}\left(\Sigma_{1}^{i}, \Sigma^{t}\right)$ & $\operatorname{dist}^{f}\left(\Sigma_{2}^{i}, \Sigma^{t}\right)$ \\
\hline 01 & DE,DER,DHP,DHPR,DP,DPR,EHP,EP & DHP,DHPR,DP,DPR \\
\hline 02 & DE,DEH,DER,DPR,PR & DPR,PR \\
\hline 03 & DE,DEH,DEHR,DER,EHP,EP & DE,DER,EHP,EP \\
\hline 04 & DE,DEH,DEP,DPR,EHPR,EHR,EP,EPR,ER,PR & DPR,EHPR,EHR,EPR,ER,PR \\
\hline 05 & DE,DEH,DPR,EHP,EHPR,EHR,EP,EPR,ER,PR & EHPR,EHR,EPR,ER \\
\hline 06 & DPR,EHP,EHPR,EP,EPR,ER,PR & EHP,EHPR,EP,EPR,ER \\
\hline 07 & DPR,EHP,EP,ER,PR & DPR,ER,PR \\
\hline 08 & DE,DEH,DEP,DPR,EHPR,EHR,EP,EPR,ER,PR & DPR,EHPR,EHR,EPR,ER,PR \\
\hline 09 & DHP,DHPR,DP,DPR,EP,EPR,PR & DHP,DHPR,DP,DPR,PR \\
\hline 10 & DHP,DHPR,DP,DPR,PR & DHP,DHPR,DP,DPR \\
\hline 11 & DE,DEH,DEHR,DER,EHP,EP & EP \\
\hline 12 & DE,DEH,DER,DPR,EHP,EHPR,EP,EPR,PR & $E P$ \\
\hline 13 & EP & $\emptyset$ \\
\hline 14 & DE,DEH,DEHP,DEHR,DEP,DER,EHP,EHPR,EP,EPR & EHP,EHPR,EP,EPR \\
\hline 15 & DE,DEH,DEP,DER,EP & EHPR,EP,EPR \\
\hline 16 & DE,DEH,DEHR,DER,DHPR,DPR,EHP,EHPR,EP,EPR,HPR,PR & DHPR,DPR,EHPR,EP,EPR,HPR,PR \\
\hline 17 & DHPR,DPR,EP,HPR,PR & $\emptyset$ \\
\hline 18 & DE,DEH,DEP,DEPR,DER,DPR,EP,EPR,PR & EP \\
\hline 19 & EP & $\emptyset$ \\
\hline 20 & DE,DEH,DEHP,DEP,DPR,EHP,EHPR,EHR,EP,EPR,ER,PR & DE,DEP,EHPR,EHR,EP,EPR,ER \\
\hline 21 & EP & $\emptyset$ \\
\hline 22 & DE,DEP,DEPR,DER,DPR,EH,EHP,EHPR,EHR,EP,EPR,ER,PR & EH,EHP,EHPR,EHR,EP,EPR,ER \\
\hline 23 & DE,DEH,DEHR,DER,DPR,EHP,EHPR,EP,EPR,PR & DPR,EHP,EHPR,EP,EPR,PR \\
\hline 24 & DE,DEH,DEHR,DER,DPR,EP,EPR,PR & DPR,EHP,EHPR,EP,EPR,PR \\
\hline 25 & DPR,EP,EPR,PR & DPR,PR \\
\hline 26 & DPR,EP,EPR,PR & DHP,DP,DPR,EP,EPR,PR \\
\hline 27 & DHPR,DPR,EHP,EP,EPR,HPR,PR & DHPR,DPR,EP,EPR,HPR,PR \\
\hline 28 & $\begin{array}{l}\text { DE,DEH,DEHP,DEP,DHP,DHPR,DHR,DP,DPR, } \\
\text { DR,EHP,EP,ER,HP,HPR,HR,P,PR,R }\end{array}$ & $\begin{array}{l}\text { DE,DEH,DEP,DHP,DHPR,DHR,DP,DPR,DR,EP, } \\
\text { ER,HP,HPR,HR,P,PR,R }\end{array}$ \\
\hline 29 & DPR,E,EH,EHP,EHPR,EHR,EP,EPR,ER,PR & E,EH,EHP,EHPR,EHR,EP,EPR,ER \\
\hline 30 & $\begin{array}{l}\text { DE,DEH,DEHP,DEP,DEPR,DER,DH,DHP,DHPR, } \\
\text { DHR,DPR,EHP,EHR,EP,EPR,ER,PR }\end{array}$ & DE,DEH,DER,DPR,EHP,EHPR,EP,EPR,PR \\
\hline 31 & DPR,E,EH,EHR,EP,EPR,ER,PR & E,EH,EHR,EP,ER \\
\hline 32 & $\begin{array}{l}\text { DE,DEH,DEHR,DER,DHP,DHPR,DP,DPR, } \\
\text { EH,EHP,EHPR,EP,EPR,HP,HPR,P,PR }\end{array}$ & $\begin{array}{l}\text { DHP,DHPR,DP,DPR,EH,EHP,EHPR,EP, } \\
\text { EPR,HP,HPR,P,PR }\end{array}$ \\
\hline 33 & DE,DEH,DEHR,DER,DR,EHP,EHPR,EP,EPR,PR & DHP,DHPR,DP,DPR,DR,EHP,EHPR,EP,EPR,PR \\
\hline 34 & DHR,DPR,DR,E,EH,EHP,EHPR,EHR,EP,EPR,ER,HR,PR,R & DPR,E,EH,EHP,EHPR,EHR,EP,EPR,ER,PR \\
\hline 35 & DE,DEH,DEHR,DER,DPR,EHP,EP,EPR,PR & DPR,EHP,EP,EPR,PR \\
\hline 36 & $\begin{array}{l}\text { DE,DEH,DEHR,DER,DHP,DHPR,DP,DPR,E,EH, } \\
\text { EHP,EHPR,EP,EPR,HP,HPR,P,PR }\end{array}$ & $\begin{array}{l}\text { DHP,DHPR,DP,DPR,E,EH,EHP,EHPR,EP,EPR, } \\
\text { HP,HPR,P,PR }\end{array}$ \\
\hline 37 & DPR,E,EH,EHP,EHPR,EHR,EP,EPR,ER,PR & E,EH,EHP,EHPR,EHR,EP,EPR,ER \\
\hline 38 & DE,DEH,DPR,EHP,EHPR,EP,EPR,ER,PR & DE,EP,ER \\
\hline 39 & DHP,DP,DPR,EHP,EP,EPR,PR & DHP,DP,EHP,EP \\
\hline 40 & $\begin{array}{l}\text { DE,DEH,DEHP,DEHR,DEP,DER,DHPR,DPR, } \\
\text { EHP,EHPR,EP,EPR,HPR,PR }\end{array}$ & DHPR,DPR,EHP,EHPR,EP,EPR,HPR,PR \\
\hline 41 & DPR,EHP,EHPR,EP,EPR,HPR,PR & EHP,EHPR,EP,EPR \\
\hline 42 & DE,DEH,DEHR,DER,DPR,EHP,EHPR,EP,EPR,PR & DE,DER,DPR,EHP,EHPR,EP,EPR,PR \\
\hline 43 & DPR,EHP,EP,EPR,PR & EP \\
\hline 44 & DPR,E,EH,EHP,EHPR,EHR,EP,EPR,ER,PR & E,EH,EHP,EHPR,EHR,EP,EPR,ER \\
\hline 45 & DHP,DHPR,DP,DPR,EP,EPR,PR & DHP,DHPR,DP,DPR,EP \\
\hline 46 & DE,DEH,DEHR,DER,DPR,E,EH,PR & DPR,E,EH,EHP,EHPR,EP,EPR,PR \\
\hline 47 & DE,DEH,DEHR,DER,DPR,EHP,EHPR,EP,EPR,PR & DPR,EHP,EHPR,EP,EPR,PR \\
\hline 48 & DE,DEH,DEHR,DER,DPR,EHP,EHPR,EP,EPR,PR & EHP,EHPR,EP,EPR \\
\hline 49 & DPR,EP,EPR,PR & EP \\
\hline 50 & DE,DEH,DEHR,DER,DPR,EHP,EHPR,EP,EPR,PR & DE,DEH,DEHR,DER,EHP,EHPR,EP,EPR \\
\hline
\end{tabular}

Table 7.20: Qualitative analysis of proximity for FDs 
and is the natural counterpart for the distance of two constraint sets. Indeed, recall from Chapter 6 that both our proximity measures define metrics.

We now list for each team $i$ the distances between their constraint sets $\Sigma_{1}^{i}, \Sigma_{2}^{i}$, and the target set $\Sigma^{t}$, separately for uniqueness constraints and functional dependencies. The analysis follows from the analyses of soundness and completeness, as

$$
\operatorname{dist}^{u}\left(\Sigma, \Sigma^{t}\right)=\operatorname{unsound}_{\Sigma^{t}}^{u}\left(\Sigma, \Sigma^{t}\right) \cup \text { incomplete }_{\Sigma^{t}}^{u}\left(\Sigma, \Sigma^{t}\right)
$$

and

$$
\operatorname{dist}^{f}\left(\Sigma, \Sigma^{t}\right)=\operatorname{unsound}_{\Sigma^{t}}^{f}\left(\Sigma, \Sigma^{t}\right) \cup \text { incomplete }_{\Sigma^{t}}^{f}\left(\Sigma, \Sigma^{t}\right)
$$

hold.

Recall from before that the inspection of Armstrong tables helps with identifying meaningful UCs and FDs but not with identifying meaningless UCs nor FDs. It is in this sense that the inspection of Armstrong tables helps with the approximation of the target set in terms of proximity. As we have seen before, there is no particular preference to which meaningful UCs or FDs are recognized, or to which meaningless UCs or FDs are not recognized.

\subsubsection{Qualitative analysis by undiscovered constraints}

In this section we move away from our formal measures and simply look at those target constraints which were undiscovered after the first phase, and which were undiscovered after the second phase. Table 7.21 lists for each of the 50 participating teams which target FDs and which target UC they did not discover after phase 1 , and which target FDs and which target UCs they did not discover after phase 2. Some of the target constraints are marked with a symbol $*$ next to them. These constraints were not explicitly specified by the teams, but they were still discovered in the sense that they were implied by the constraint sets specified. 


\begin{tabular}{|c|c|c|c|c|}
\hline \multirow[b]{2}{*}{ Team } & \multicolumn{2}{|c|}{ After phase 1} & \multicolumn{2}{|c|}{ After phase 2} \\
\hline & Undiscovered FDs & Undiscovered UCS & Undiscovered FDs & Undiscovered UCS \\
\hline CSAU01 & $\mathrm{PR} \rightarrow \mathrm{H}$ & - & - & - \\
\hline CSAU02 & $\mathrm{EP} \rightarrow \mathrm{H}, \mathrm{EP} \rightarrow \mathrm{R}$ & $\mathrm{u}(\mathrm{DE})$ & $\mathrm{EP} \rightarrow \mathrm{H}$ & - \\
\hline CSAU03 & $\mathrm{EP} \rightarrow \mathrm{H}^{*}, \mathrm{PR} \rightarrow \mathrm{H}$ & $\mathrm{u}(\mathrm{DE})$ & $\mathrm{EP} \rightarrow \mathrm{H}$ & $\mathrm{u}(\mathrm{DE})$ \\
\hline CSAU04 & $\mathrm{EP} \rightarrow \mathrm{H}$ & - & - & - \\
\hline CSAU05 & $\mathrm{EP} \rightarrow \mathrm{H}, \mathrm{EP} \rightarrow \mathrm{R}$ & $\mathrm{u}(\mathrm{DE})$ & - & - \\
\hline CSAU06 & $\mathrm{EP} \rightarrow \mathrm{H}$ & - & $\mathrm{EP} \rightarrow \mathrm{H}$ & - \\
\hline CSAU07 & $\mathrm{EP} \rightarrow \mathrm{H}, \mathrm{EP} \rightarrow \mathrm{R}$ & $\mathrm{u}(\mathrm{DE})$ & $\mathrm{EP} \rightarrow \mathrm{H}$ & - \\
\hline CSAU08 & $\mathrm{EP} \rightarrow \mathrm{H}$ & - & - & - \\
\hline CSAU09 & $\mathrm{EP} \rightarrow \mathrm{H}, \mathrm{PR} \rightarrow \mathrm{H}$ & $\mathrm{u}(\mathrm{DE})$ & $\mathrm{EP} \rightarrow \mathrm{H}$ & - \\
\hline CSAU10 & $\mathrm{EP} \rightarrow \mathrm{H}$ & - & - & - \\
\hline CSAU11 & $\mathrm{EP} \rightarrow \mathrm{H}, \mathrm{EP} \rightarrow \mathrm{R}, \mathrm{PR} \rightarrow \mathrm{H}$ & $\mathrm{u}(\mathrm{DE})$ & $\mathrm{EP} \rightarrow \mathrm{H}$ & $\mathrm{u}(\mathrm{DE})$ \\
\hline CSAU12 & $\mathrm{EP} \rightarrow \mathrm{H}$ & - & - & - \\
\hline CSVUW01 & $\mathrm{EP} \rightarrow \mathrm{H}, \mathrm{EP} \rightarrow \mathrm{R}, \mathrm{PR} \rightarrow \mathrm{H}$ & $\mathrm{u}(\mathrm{DE})$ & $\mathrm{EP} \rightarrow \mathrm{H}^{*}, \mathrm{EP} \rightarrow \mathrm{R}^{*}$ & - \\
\hline CSVUW02 & $\mathrm{EP} \rightarrow \mathrm{H}, \mathrm{PR} \rightarrow \mathrm{H}$ & $\mathrm{u}(\mathrm{DE})$ & $\mathrm{PR} \rightarrow \mathrm{H}$ & - \\
\hline CSVUW03 & $\mathrm{EP} \rightarrow \mathrm{H}, \mathrm{PR} \rightarrow \mathrm{H}$ & $\mathrm{u}(\mathrm{DE})$ & $\mathrm{PR} \rightarrow \mathrm{H}, \mathrm{EP} \rightarrow \mathrm{H}^{*}$ & - \\
\hline CSVUW04 & $\mathrm{EP} \rightarrow \mathrm{H}, \mathrm{PR} \rightarrow \mathrm{H}$ & - & $\mathrm{PR} \rightarrow \mathrm{H}$ & - \\
\hline CSVUW05 & $\mathrm{EP} \rightarrow \mathrm{H}, \mathrm{PR} \rightarrow \mathrm{H}$ & - & $\mathrm{PR} \rightarrow \mathrm{H}, \mathrm{EP} \rightarrow \mathrm{H}$ & - \\
\hline CSVUW06 & $\mathrm{EP} \rightarrow \mathrm{H}, \mathrm{EP} \rightarrow \mathrm{R}, \mathrm{PR} \rightarrow \mathrm{H}$ & - & $\mathrm{EP} \rightarrow \mathrm{H}, \mathrm{PR} \rightarrow \mathrm{H}$ & - \\
\hline CSVUW07 & $\mathrm{EP} \rightarrow \mathrm{H}, \mathrm{EP} \rightarrow \mathrm{R}, \mathrm{PR} \rightarrow \mathrm{H}^{*}$ & $\mathrm{u}(\mathrm{DE})$ & $\mathrm{EP} \rightarrow \mathrm{H}, \mathrm{PR} \rightarrow \mathrm{H}^{*}$ & $\mathrm{u}(\mathrm{DE})$ \\
\hline CSVUW08 & $\mathrm{EP} \rightarrow \mathrm{H}^{*}, \mathrm{EP} \rightarrow \mathrm{R}^{*}, \mathrm{PR} \rightarrow \mathrm{H}$ & - & $\mathrm{EP} \rightarrow \mathrm{H}^{*}, \mathrm{EP} \rightarrow \mathrm{R}^{*}$ & - \\
\hline CSVUW09 & $\mathrm{EP} \rightarrow \mathrm{H}, \mathrm{EP} \rightarrow \mathrm{R}, \mathrm{PR} \rightarrow \mathrm{H}$ & $\mathrm{u}(\mathrm{DE})$ & $\mathrm{EP} \rightarrow \mathrm{H}^{*}, \mathrm{PR} \rightarrow \mathrm{H}$ & $\mathrm{u}(\mathrm{DE})$ \\
\hline CSVUW10 & $\mathrm{EP} \rightarrow \mathrm{H}, \mathrm{PR} \rightarrow \mathrm{H}$ & - & $\mathrm{EP} \rightarrow \mathrm{H}, \mathrm{EP} \rightarrow \mathrm{R}^{*}$ & - \\
\hline CSVUW11 & $\mathrm{EP} \rightarrow \mathrm{H}^{*}, \mathrm{EP} \rightarrow \mathrm{R}^{*}, \mathrm{PR} \rightarrow \mathrm{H}^{*}$ & $\mathrm{u}(\mathrm{DE})$ & $\mathrm{PR} \rightarrow \mathrm{H}^{*}$ & - \\
\hline CSVUW12 & $\mathrm{EP} \rightarrow \mathrm{H}^{*}, \mathrm{EP} \rightarrow \mathrm{R}^{*}, \mathrm{PR} \rightarrow \mathrm{H}$ & $\mathrm{u}(\mathrm{DE})$ & $\mathrm{EP} \rightarrow \mathrm{H}^{*}, \mathrm{PR} \rightarrow \mathrm{H}$ & - \\
\hline CSVUW13 & $\mathrm{EP} \rightarrow \mathrm{H}^{*}, \mathrm{EP} \rightarrow \mathrm{R}^{*}, \mathrm{PR} \rightarrow \mathrm{H}$ & $\mathrm{u}(\mathrm{DE})^{*}$ & $\mathrm{EP} \rightarrow \mathrm{H}^{*}, \mathrm{EP} \rightarrow \mathrm{R}^{*}, \mathrm{PR} \rightarrow \mathrm{H}$ & $\mathrm{u}(\mathrm{DE})^{*}$ \\
\hline CSVUW14 & $\mathrm{EP} \rightarrow \mathrm{H}, \mathrm{EP} \rightarrow \mathrm{R}, \mathrm{PR} \rightarrow \mathrm{H}$ & $\mathrm{u}(\mathrm{DE})$ & $\mathrm{EP} \rightarrow \mathrm{H}, \mathrm{EP} \rightarrow \mathrm{R}, \mathrm{PR} \rightarrow \mathrm{H}$ & - \\
\hline CSVUW15 & $\mathrm{EP} \rightarrow \mathrm{H}^{*}, \mathrm{EP} \rightarrow \mathrm{R}^{*}, \mathrm{PR} \rightarrow \mathrm{H}^{*}$ & $\mathrm{u}(\mathrm{DE})$ & $\mathrm{EP} \rightarrow \mathrm{H}^{*}, \mathrm{PR} \rightarrow \mathrm{H}^{*}$ & - \\
\hline CSVUW16 & $\mathrm{EP} \rightarrow \mathrm{H}^{*}, \mathrm{EP} \rightarrow \mathrm{R}^{*}, \mathrm{PR} \rightarrow \mathrm{H}$ & $\mathrm{u}(\mathrm{DE})^{*}$ & $\mathrm{EP} \rightarrow \mathrm{H}^{*}, \mathrm{EP} \rightarrow \mathrm{R}^{*}$ & $\mathrm{u}(\mathrm{DE})^{*}$ \\
\hline ISVUW01 & $\mathrm{EP} \rightarrow \mathrm{H}, \mathrm{EP} \rightarrow \mathrm{R}$ & $\mathrm{u}(\mathrm{DE})$ & - & - \\
\hline ISVUW02 & $\mathrm{PR} \rightarrow \mathrm{H}$ & $\mathrm{u}(\mathrm{DE})$ & $\mathrm{PR} \rightarrow \mathrm{H}$ & - \\
\hline ISVUW03 & $\mathrm{EP} \rightarrow \mathrm{H}, \mathrm{EP} \rightarrow \mathrm{R}$ & $\mathrm{u}(\mathrm{DE})$ & $\mathrm{EP} \rightarrow \mathrm{R}$ & $\mathrm{u}(\mathrm{DE})$ \\
\hline ISVUW04 & $\mathrm{EP} \rightarrow \mathrm{H}, \mathrm{PR} \rightarrow \mathrm{H}$ & $\mathrm{u}(\mathrm{DE})$ & $\mathrm{PR} \rightarrow \mathrm{H}$ & - \\
\hline ISVUW05 & $\mathrm{EP} \rightarrow \mathrm{R}, \mathrm{PR} \rightarrow \mathrm{H}$ & $\mathrm{u}(\mathrm{DE})$ & - & - \\
\hline ISVUW06 & $\mathrm{EP} \rightarrow \mathrm{H}^{*}, \mathrm{EP} \rightarrow \mathrm{R}^{*}, \mathrm{PR} \rightarrow \mathrm{H}$ & - & $\mathrm{EP} \rightarrow \mathrm{H}^{*}$ & - \\
\hline CSLU01 & $\mathrm{EP} \rightarrow \mathrm{H}, \mathrm{EP} \rightarrow \mathrm{R}, \mathrm{PR} \rightarrow \mathrm{H}$ & - & $\mathrm{PR} \rightarrow \mathrm{H}$ & - \\
\hline CSLU02 & $\mathrm{EP} \rightarrow \mathrm{H}, \mathrm{PR} \rightarrow \mathrm{H}$ & $\mathrm{u}(\mathrm{DE})$ & $\mathrm{PR} \rightarrow \mathrm{H}$ & - \\
\hline CSLU03 & $\mathrm{EP} \rightarrow \mathrm{H}, \mathrm{PR} \rightarrow \mathrm{H}$ & - & $\mathrm{PR} \rightarrow \mathrm{H}$ & - \\
\hline CSLU04 & $\mathrm{EP} \rightarrow \mathrm{H}^{*}, \mathrm{EP} \rightarrow \mathrm{R}^{*}, \mathrm{PR} \rightarrow \mathrm{H}$ & $\mathrm{u}(\mathrm{DE})$ & $\mathrm{EP} \rightarrow \mathrm{H}$ & $\mathrm{u}(\mathrm{DE})$ \\
\hline CSLU05 & $\mathrm{EP} \rightarrow \mathrm{H}, \mathrm{EP} \rightarrow \mathrm{R}, \mathrm{PR} \rightarrow \mathrm{H}$ & - & $\mathrm{EP} \rightarrow \mathrm{H}, \mathrm{EP} \rightarrow \mathrm{R}$ & - \\
\hline CSLU06 & $\mathrm{EP} \rightarrow \mathrm{R}, \mathrm{PR} \rightarrow \mathrm{H}^{*}$ & $\mathrm{u}(\mathrm{DE})$ & $\mathrm{PR} \rightarrow \mathrm{H}^{*}$ & - \\
\hline CSLU07 & $\mathrm{EP} \rightarrow \mathrm{R}, \mathrm{PR} \rightarrow \mathrm{H}$ & - & $\mathrm{EP} \rightarrow \mathrm{R}^{*}$ & - \\
\hline CSLU08 & $\mathrm{EP} \rightarrow \mathrm{R}, \mathrm{PR} \rightarrow \mathrm{H}$ & $\mathrm{u}(\mathrm{DE})$ & $\mathrm{EP} \rightarrow \mathrm{R}, \mathrm{PR} \rightarrow \mathrm{H}$ & $\mathrm{u}(\mathrm{DE})$ \\
\hline CSLU09 & $\mathrm{EP} \rightarrow \mathrm{H}, \mathrm{EP} \rightarrow \mathrm{R}, \mathrm{PR} \rightarrow \mathrm{H}$ & - & $\mathrm{EP} \rightarrow \mathrm{H}$ & - \\
\hline CSLU10 & $\mathrm{EP} \rightarrow \mathrm{H}^{*}, \mathrm{EP} \rightarrow \mathrm{R}^{*}, \mathrm{PR} \rightarrow \mathrm{H}$ & $\mathrm{u}(\mathrm{DE})^{*}$ & $\mathrm{EP} \rightarrow \mathrm{H}^{*}$ & $\mathrm{u}(\mathrm{DE})^{*}$ \\
\hline CSLU11 & $\mathrm{EP} \rightarrow \mathrm{H}, \mathrm{PR} \rightarrow \mathrm{H}$ & - & $\mathrm{EP} \rightarrow \mathrm{H}$ & - \\
\hline CSLU12 & $\mathrm{EP} \rightarrow \mathrm{R}^{*}, \mathrm{PR} \rightarrow \mathrm{H}$ & $\mathrm{u}(\mathrm{DE})$ & $\mathrm{EP} \rightarrow \mathrm{R}^{*}, \mathrm{PR} \rightarrow \mathrm{H}$ & - \\
\hline CSLU13 & $\mathrm{EP} \rightarrow \mathrm{H}^{*}, \mathrm{EP} \rightarrow \mathrm{R}^{*}, \mathrm{PR} \rightarrow \mathrm{H}$ & $\mathrm{u}(\mathrm{DE})$ & $\mathrm{EP} \rightarrow \mathrm{H}^{*}, \mathrm{EP} \rightarrow \mathrm{R}^{*}, \mathrm{PR} \rightarrow \mathrm{H}$ & - \\
\hline CSLU14 & $\mathrm{PR} \rightarrow \mathrm{H}$ & $\mathrm{u}(\mathrm{DE})$ & - & - \\
\hline CSLU15 & $\mathrm{EP} \rightarrow \mathrm{H}, \mathrm{PR} \rightarrow \mathrm{H}$ & - & $\mathrm{EP} \rightarrow \mathrm{H}$ & - \\
\hline CSLU16 & $\mathrm{EP} \rightarrow \mathrm{H}^{*}, \mathrm{EP} \rightarrow \mathrm{R}^{*}, \mathrm{PR} \rightarrow \mathrm{H}$ & $\mathrm{u}(\mathrm{DE})$ & $\mathrm{EP} \rightarrow \mathrm{H}^{*}, \mathrm{EP} \rightarrow \mathrm{R}^{*}$ & $\mathrm{u}(\mathrm{DE})$ \\
\hline
\end{tabular}

Table 7.21: Undiscovered UCs and FDs before and after using Armstrong tables. The symbol $*$ next to a constraint indicates that it was not explicitly mentioned by the team but implied by its constraint set 
The results of Table 7.21 are summarized in Table 7.22, It shows that 56 percent of all target constraints remained undiscovered after phase 1 , while only 22 percent remained undiscovered after inspecting Armstrong tables. Therefore, Armstrong tables enabled the teams to discover 34 percent more of the target constraints. In total, 68 more constraints were discovered with the help of Armstrong tables, leading from 112 undiscovered constraints after phase 1 to 44 undiscovered constraints after phase 2 .

\begin{tabular}{|ccc|}
\hline & After phase 1 & After phase 2 \\
\hline $\mathrm{EP} \rightarrow \mathrm{H}$ & $30 / 50$ & $16 / 50$ \\
$\mathrm{PR} \rightarrow \mathrm{H}$ & $36 / 50$ & $16 / 50$ \\
$\mathrm{EP} \rightarrow \mathrm{R}$ & $18 / 50$ & $4 / 50$ \\
$\mathrm{u}(\mathrm{DE})$ & $28 / 50$ & $8 / 50$ \\
\hline Total & $112 / 200$ & $44 / 200$ \\
In percent & 56 & 22 \\
\hline
\end{tabular}

Table 7.22: Number of teams that did not discover a given target constraint

Armstrong tables provided the most help with the discovery of the FD $P R \rightarrow H$ and the UC $u(D E)$. Indeed, 20 teams discovered these constraints after phase 2, while they had not discovered these constraints after phase 1 . For the FDs $E P \rightarrow H$ and $E P \rightarrow R$ there were 14 teams who discovered these constraints only with the help of Armstrong tables.

\subsubsection{Qualitative analysis per groups}

For the qualitative analysis per groups we simply break down Table 7.22 from the previous subsection into groups. Indeed, Tables 7.23 and 7.24 summarize the results from Table 7.21 per group.

Table 7.23 shows that the group CS AU discovered the most constraints amongst all groups without inspecting an Armstrong table. In fact, they discovered half of all the target constraints possible. They were followed by the group CS LU with 44 percent of possible target constraints discov- 


\begin{tabular}{|c|cccccc|}
\hline & \multicolumn{5}{|c|}{ After phase 1 } \\
\cline { 2 - 7 } Group & $\mathrm{EP} \rightarrow \mathrm{H}$ & $\mathrm{PR} \rightarrow \mathrm{H}$ & $\mathrm{EP} \rightarrow \mathrm{R}$ & $\mathrm{u}(\mathrm{DE})$ & Total & In Percent \\
\hline CS AU & $10 / 12$ & $4 / 12$ & $4 / 12$ & $6 / 12$ & $24 / 48$ & 50 \\
CS VUW & $10 / 16$ & $13 / 16$ & $5 / 16$ & $9 / 16$ & $37 / 64$ & 58 \\
IS VUW & $3 / 6$ & $4 / 6$ & $3 / 6$ & $5 / 6$ & $15 / 24$ & 63 \\
CS LU & $7 / 16$ & $15 / 16$ & $6 / 16$ & $8 / 16$ & $36 / 64$ & 56 \\
\hline
\end{tabular}

Table 7.23: Number of teams per group that did not discover a given target constraint after phase 1

\begin{tabular}{|c|cccccc|}
\hline & \multicolumn{7}{|c|}{ After phase 2 } \\
\cline { 2 - 7 } Group & $\mathrm{EP} \rightarrow \mathrm{H}$ & $\mathrm{PR} \rightarrow \mathrm{H}$ & $\mathrm{EP} \rightarrow \mathrm{R}$ & $\mathrm{u}(\mathrm{DE})$ & Total & In Percent \\
\hline CS AU & $6 / 12$ & $0 / 12$ & $0 / 12$ & $2 / 12$ & $8 / 48$ & 17 \\
CS VUW & $5 / 16$ & $8 / 16$ & $1 / 16$ & $2 / 16$ & $16 / 64$ & 25 \\
IS VUW & $0 / 6$ & $2 / 6$ & $1 / 6$ & $1 / 6$ & $4 / 24$ & 17 \\
CS LU & $5 / 16$ & $6 / 16$ & $2 / 16$ & $3 / 16$ & $16 / 64$ & 25 \\
\hline
\end{tabular}

Table 7.24: Number of teams per group that did not discover a given target constraint after phase 2

\begin{tabular}{|c|c|c|c|c|c|c|c|c|c|c|c|c|}
\hline & \multicolumn{3}{|c|}{$\mathrm{EP} \rightarrow \mathrm{H}$} & \multicolumn{3}{|c|}{$\mathrm{PR} \rightarrow \mathrm{H}$} & \multicolumn{3}{c|}{$\mathrm{EP} \rightarrow \mathrm{R}$} & \multicolumn{3}{c|}{$\mathrm{u}(\mathrm{DE})$} \\
\cline { 2 - 13 } Group & P1 & P2 & P2-P1 & P1 & P2 & P2-P1 & P1 & P2 & P2-P1 & P1 & P2 & P2-P1 \\
\hline CS AU & 17 & 50 & 33 & 67 & 100 & 33 & 67 & 100 & 33 & 50 & 83 & 33 \\
CS VUW & 38 & 69 & 31 & 19 & 50 & 31 & 69 & 94 & 25 & 44 & 88 & 44 \\
IS VUW & 50 & 100 & 50 & 33 & 66 & 33 & 50 & 83 & 33 & 17 & 83 & 66 \\
CS LU & 56 & 69 & 13 & 6 & 63 & 57 & 63 & 88 & 25 & 50 & 81 & 31 \\
\hline
\end{tabular}

Table 7.25: Percentage of teams per group that discovered a given target constraint after phase 1 (P1) and phase 2 (P2) and their difference (P2-P1) 
ered, the group CS VUW with 42 percent, and the group IS VUW with 37 percent discovered. Table 7.24 shows that the groups CS AU and IS VUW discovered the most constraints amongst all groups after inspecting an Armstrong table. In fact, they discovered 83 percent of all the target constraints possible. They were followed by the groups CS LU and CS VUW with 75 percent of possible target constraints discovered.

This means that the inspection of Armstrong tables had the biggest positive effect on the group IS VUW who increased the discovery of target constraints by 46 percent, followed by the groups CS VUW and CS AU who both had an increase of 33 percent, and the group CS LU who had an increase of 31 percent.

Finally, we look at Table 7.25, which shows per group, the percentages of teams that did discover a given target constraint after phase 1 (P1) and phase 2 (P2), respectively, and the difference of these percentages (P2-P1).

For $E P \rightarrow H$, we observe that the group CS LU had the most teams who discovered this FD after phase 1, but only all teams of the group IS VUW discovered the FD after phase 2. The same group also showed the biggest learning effect for this FD from inspecting Armstrong tables.

For $P R \rightarrow H$, we observe that the group CS AU had the most teams who discovered this FD after phase 1, and only all teams of this group discovered the FD after phase 2. The group CS LU showed the biggest learning effect for this FD from inspecting Armstrong tables.

For $E P \rightarrow R$, we observe that the group CS VUW had the most teams who discovered this FD after phase 1, and only all teams of the group CS AU discovered the FD after phase 2. The groups CS AU and IS VUW showed the biggest learning effect for this FD from inspecting Armstrong tables.

For $u(D E)$, the groups CS AU and CS LU had the most teams who discovered the UC after phase 1, and the group CS VUW had the most teams who discovered the UC after phase 2. The group IS VUW showed the biggest learning effect for the UC from inspecting Armstrong tables. 
Amongst all groups, the biggest average learning effect from inspecting Armstrong tables was observed for the $u(D E)$ with 44 percent of more teams discovering the UC, followed by the FD $P R \rightarrow H$ with 39 percent more teams, then the FD $E P \rightarrow H$ with 32 percent, and finally the FD $E P \rightarrow R$ with 29 percent.

\subsection{Summary}

The quantitative and qualitative analysis of the data, collected from 50 teams at different universities, suggests that Armstrong tables are i) effective in terms of completeness, ii) ineffective in terms of soundness, and thereby iii) useful in terms of proximity. Our relative measures allow us to say that the violation of meaningful uniqueness constraints and functional dependencies is rather certain to be observed when inspecting Armstrong tables. Moreover, the satisfaction of meaningless uniqueness constraints and functional dependencies is rather impossible to be observed when inspecting Armstrong tables. Our data confirms that these observations are robust under different meaningless and different meaningful uniqueness constraints and functional dependencies, and different educational and cultural backgrounds. The analysis confirms, in particular, that the tool SQL-Sampler produced Armstrong tables for which these observations hold. In summary, SQL-Sampler produces Armstrong tables that are of great value for the acquisition of meaningful uniqueness constraints and functional dependencies that encode application semantics in the form of SQL constraints. For practice, our conclusions suggest to recommend the use of Armstrong tables as early as possible in the requirements acquisition process. On the one hand, this is to prevent meaningless constraints from being incorrectly perceived as meaningful and not being able to recognize their meaningless later on in the process. On the other hand, Armstrong tables are likely to produce a more complete set of semantically meaningful constraints, probably also with less resources involved. 


\section{Chapter 8}

\section{Conclusion and Future Work}

In this final chapter the contributions of the thesis are summarized and avenues for future possible work are discussed briefly.

\subsection{Conclusion}

Functional dependencies play a central role for the design [24, 54, 105, 107 , 123, 124] and normalization [90, 106] of databases, and have found important applications in a diverse number of areas including query optimization [27, 39, 104], database maintenance [53, 74, 118] and security [14, 132], and data cleaning [51, 52], entry [105], exchange [98] and integration [23,101,131]. Fundamental to all these applications are the assumptions that $i)$ the set of functional dependencies that are semantically meaningful for a given relation schema has been correctly identified, and ii) the underlying data is purely relational.

The research of this thesis establishes new contributions that enable database designers to recognize a more complete set of semantically meaningful functional dependencies where the underlying data may contain duplicate rows and null marker occurrences.

As a first contribution, the thesis has developed new foundations for the combined class of uniqueness constraints, NOT NULL constraints and 
functional dependencies in databases that may contain duplicate rows and null marker occurrences of the type value unknown at present. More precisely, the associated implication problem has been characterized axiomatically by a finite set of inference rules, by an input-linear time algorithm, and in terms of Horn clauses interpreted in the well-known approximation logic $\mathcal{S}$-3. The structure of Armstrong tables for this combined class of constraints has then been characterized in terms of maximal, duplicate and closed sets. The structural characterization was exploited to establish a provably-correct algorithm that computes an Armstrong table for an arbitrarily given finite set of constraints from this class. While the problem of finding an Armstrong table is precisely exponential, the algorithm computes an Armstrong table that is guaranteed to have a number of rows that is at most quadratic in the minimum number of rows required for the input.

As a second contribution, the thesis described the implementation of a toolbox for Armstrong tables, called SQL-Sampler, which comes in two flavors: either a desktop-based or a Web-based graphical user interface. SQL-Sampler allows its users to select different classes of SQL constraints, such as the combined class above under the value unknown at present interpretation of null markers, but also the same combined class under the no information interpretation. The tool allows its user to specify a table schema, a null-free subschema, and a set of uniqueness constraints and/or functional dependencies, and then computes an Armstrong table for this input. The tables are populated with either artificial values or values predefined by the users of the tool. In the first case, users can also replace the artificial values by other values.

As a third contribution, the thesis investigated empirically in which sense Armstrong tables, as produced by SQL-Sampler, are useful. For this purpose, the focus was shifted towards the combined class of uniqueness constraints, NOT NULL constraints and functional dependencies under the no information interpretation. Several measures such as soundness, com- 
pleteness and proximity were introduced to formalize the notion of "usefulness". Detailed experiments with 50 design teams suggested that, by inspecting Armstrong tables, database designers are likely to recognize the actual meaningfulness of most uniqueness constraints and functional dependencies that they perceived as meaningless prior to the inspection. The experiments also suggested that, by inspecting Armstrong tables, database designers are unlikely to recognize the actual meaninglessness of most uniqueness constraints and functional dependencies that they perceived as meaningful prior to the inspection. These empirical observations are consistent with the structural properties of Armstrong tables.

The research extends several well-known findings from the idealized special case of relations to tables, where duplicate rows and null marker occurrences are permitted to occur.

In conclusion, the contributions of the thesis have given detailed answers to the following research question: How can database designers discover real-world database constraints that are meaningful for the given application domain?

\subsection{Future Work}

The research naturally suggests a variety of open problems that are interesting to consider in future work.

On the foundation side, one may consider other popular classes of database constraints. It might be interesting to start with multivalued dependencies, since these naturally extend the class of functional dependencies and since there already is known work in the presence of no information null markers [70, 89]. However, the author is unaware of any work on multivalued dependencies under the value exists but unknown interpretation, and Armstrong databases for multivalued dependencies have not received any attention yet in the context of partial data. Multivalued dependencies also have strong links with classes of conditional 
independencies in the fields of multivariate statistics and probability theory [58,93]. Another important class of data dependencies to consider are fragments of inclusion dependencies. In general, the implication problem for the combined class of functional and inclusion dependencies is different in the context of finite databases from that in the context of unrestricted databases, and both versions are undecidable and not axiomatizable by a finite set of Horn rules [25, 102]. However, efficiently decidable fragments are known such as functional dependencies and unary inclusion dependencies. It is also known that Armstrong databases exist for the combined class of functional and inclusion dependencies over purely relational databases [50]. Inclusion dependencies would be interesting for a number of reasons. One reason is that they constitute an important class of constraints that can enforce the relationship between data across different tables. This is the reason why they also enjoy native support in real database management systems, at least in the form of foreign keys. Another reason is that inclusion dependencies can be given different semantics in the context of partial data. The SQL standard, for example, proposes the use of a simple and a partial semantics for referential integrity constraints. The author is unaware of any work on referential integrity constraints under the no information interpretation. Levene and Loizou have studied inclusion dependencies under the value exists but unknown interpretation, but not in the presence of NOT NULL constraints.

Mainstream research has recently focused on Web data models such as the eXtensible Markup Language (XML) and the Resource Description Framework (RDF). The work on XML constraints is rather advanced [5, 6, 21, 67, 68, 76, 129, 130], but it would still be interesting to investigate which impact the work on integrity constraints in the presence of partial data can have on XML constraints. The work on integrity constraints in RDF is still very much in its infancy [79, 80, 103]. The same can also be said about constraints on probabilistic data [38, 109, 117].

It would also be interesting to consider other approaches to partial 
data, including other interpretations of null markers [29, 60, 62, 95], orrelations [71,73], fuzzy [113] and rough sets [135].

On the application side, the impact of the results established in this thesis could be studied with respect to query optimization, database normalization and security, data cleaning, exchange and integration. While this has been done extensively for functional dependencies in strictly relational databases, only little work has been done in the more realistic context of incomplete data. 
Appendices 



\section{Appendix A}

\section{Human Ethics Approval}

The following documents are our human ethics application for conducting experiments approved by Human Ethics Committee of the School of Information Management at Victoria University of Wellington, New Zealand. 


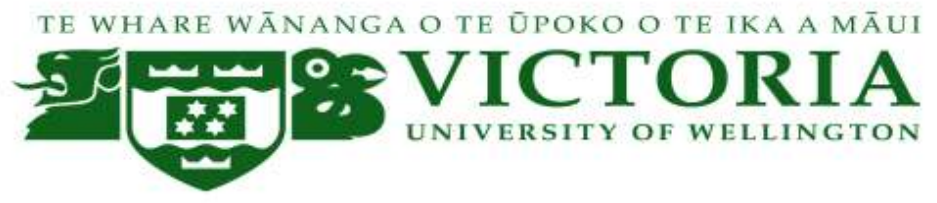

SIM HUMAN ETHICS COMMITTEE

Application for Approval of Research Projects

Please email applications to your supervisor, who will then email it to a SIM HEC member for a preliminary review.

Note: The Human Ethics Committee attempts to have all applications approved within 6 working days, but a longer period may be necessary if applications require substantial revision.

1 NATURE OF PROPOSED RESEARCH:

(a) Student Research $\quad$ Yes

(b) If Student Research Degree: PhD Course Code: INF0690

(c) Project Title: Discovering semantically meaningful constraints from sample data - Foundations, algorithms and evaluation

2 INVESTIGATORS:

(a) Principal Investigator

Name:

Van Tran Bao Le

E-mail address:

van.t.le@vuw.ac.nz

School/Dept/Group:

Information Management

(b) Other Researchers

None

(c) Supervisor (in the case of student research projects)

Dr. David Mason

A.Prof. Sebastian Link and Dr. Flavio Ferrarotti

\section{DURATION OF RESEARCH}

(a) Proposed starting date for data collection- After HEC approval has been granted (Note: that NO part of the research requiring ethical approval may commence prior to approval being given)

15 March 2012

(b) Proposed date of completion of project as a whole

28 February 20014 

CONSIDERATIONS

(a) Sources of funding for the project

Please indicate any ethical issues or conflicts of interest that may arise because of sources of funding

e.g. restrictions on publication of results

N/A

(b) Is any professional code of ethics to be followed

$\mathbf{N}$

(c) Is ethical approval required from any other body

N

If yes, name and indicate when/if approval will be given

DETAILS OF PROJECT

Briefly Outline:

(a) The objectives of the project

Armstrong databases are sample data that can represent precisely database constraints. They are regarded as a useful tool for database designers to acquire the semantics of the target database. Surprisingly, the existing theory of Armstrong databases has ignored SQL data where null marker occurrences and duplicate rows can occur. Therefore, the objective of this research is to develop a toolbox which can generate Armstrong databases for a given set of constraints on SQL data, and to verify the toolbox's effectiveness in the discovery of target database's semantics.

(b) Method of data collection

Data will be collected from Victoria University's IS students and the University of Auckland's CS students. The participants are randomly grouped into different and independent teams, and act as database designers.

Data will be gathered in two phases. In the first phase, the teams will be given a database schema of a fixed application domain (an employee example, see experimental sheet attached). The intended semantics of the database schema is verbally explained to them in English language. Each team is then given the task to write down the set of SQL constraints that they perceive as meaningful. The design teams will hand over their constraint sets to the researcher and her supervisor at the end of the first phase.

At the beginning of the second phase, each design team will be given an Armstrong table, which is produced by the toolbox, with respect to the constraint set they perceived meaningful in the first phase. The design teams will be instructed that this is a sample table that is an exact representation of their constraint set. The design teams will then be asked to revise their constraint sets with the additional help of the sample table they were given. Eventually, each team will hand in their revised constraint sets. 
Overall, for each team we will collect one constraint set after each phase. These sets, for each team individually, will be analysed subsequently. In simple terms, the toolbox is effective, if the teams can identify additional semantically meaningful SQL constraints after inspecting Armstrong tables. Precise measures have been developed for the analysis already.

\section{(c) The benefits and scientific value of the project}

As a first contribution, the existing theory of Armstrong relations will be extended to SQL databases - the industry standard. As a second contribution, the algorithms to compute Armstrong tables have been implemented in a toolbox. This can be used by database designers and data engineers to consolidate, convey, judge, test, validate, and visualize their understanding of the semantics of an application domain. The toolbox can also be used by database educators to provide automated feedback to database students. The measures defined for the evaluation of the toolbox can also be used by educators to provide automated assessment of non-multiple choice question. Therefore, the time saved for marking can be spent on discussion and interaction with students. As a final contribution, actual evidence for the effectiveness of the use of Armstrong tables will be provided. This will motivate its use for practice, and provide further insights.

\section{(d) Characteristics of the participants}

The participants do not need to have any knowledge about Armstrong relations since they will only use it as a tool. Since the participants will act as database designers, we require them to have a solid knowledge about relational databases and, in particular, SQL constraints. Therefore, students of database courses have an appropriate level of knowledge, once they are in the final stages of their course.

\section{(e) Method of recruitment}

We will ask for written consent from the course co-ordinators to use a tutorial slot to conduct our experiments. The students that attend these tutorials will be given the choice to participate, and will be asked for their written consent prior to the beginning of the experiment. It will also be made clear explicitly clear that we assess the toolbox and not the students, and that their (non-) participation in the project does not have any impact on the evaluation of their performance in the database courses. We will also point out that it is a useful exercise for them to do, and they can gain good insights from it. These points have been acknowledged by the supervisor in writing. The average enrolment number for database courses in Victoria and Auckland together is around 200 students. Since we aim for data sets from about 20 teams, and since each team consists of 3 members, there will be a sufficient number of students. 
(g) Other assistance (e.g. meals, transport) that is to be given to participants

(h) Any special hazards and/or inconvenience (including deception) that participants will encounter

None

(i) State whether consent is for: (Please indicate as many as it applies)

(i) the collection of data

(ii) attribution of opinions or information

(iii) release of data to others

(iv) use for a conference report or a publication

(v) use for some particular purpose (specify)

(INF0690- PhD Thesis)

Attach a copy of any questionnaire or interview schedule to the application

(j) How is informed consent to be obtained (see paragraphs 4.31(g), 5.2, 5.5 and 5.61 of the Guidelines)

(i) the research is strictly anonymous, an information sheet is supplied and informed consent is implied by voluntary participation in filling out a questionnaire for example (include a copy of the information sheet)

(ii) the research is not anonymous but is confidential and informed consent will be obtained through a signed consent form (include a copy of the consent form and information sheet)

(iii) the research is neither anonymous nor confidential and informed consent will be obtained through a signed consent form (include a copy of the consent form and information sheet)

(iv) informed consent will be obtained by some other method (please specify and provide details)

With the exception of anonymous research as in (i), if it is proposed that written consent will not be obtained, please explain why

(k) If the research will not be conducted on a strictly anonymous basis state how issues of confidentiality of participants are to be ensured if this is intended. (See paragraph 4.3.1(e) of the Guidelines). (e.g. who will listen to tapes, see questionnaires or have access to data). Please ensure that you distinguish clearly between anonymity and confidentiality. Indicate which of these are applicable.

(i) access to the research data will be restricted to the investigator

(ii) access to the research data will be restricted to the investigator and their supervisor (student research) 
(iii) all opinions and data will be reported in aggregated form in such a way that individual persons or organisations are not identifiable

(iv) Other (please specify)

N/A

(I) Procedure for the storage of, access to and disposal of data, both during and at the conclusion of the research. (see section 7 of the guidelines). Indicate which are applicable:

(i) all written material (questionnaires, interview notes, etc) will be kept in a locked file and access is restricted to the investigator $\quad \mathbf{Y}$

(ii) all electronic information will be kept in a password-protected file and access will be restricted to the investigator $\quad Y$

(iii) all questionnaires, interview notes and similar materials will be destroyed:

(a) at the conclusion of the research

or (b) 2 years after the conclusion of the research

(iv) any audio or video recordings will be returned to participants and/or electronically wiped

(v) other procedures (please specify):

N/A

(m)Feedback procedures (See section 8 of the Guidelines). You should indicate whether feedback will be provided to participants and in what form. If feedback will not be given, indicate the reasons why.

Feedback will be given after the completion of the experiment, indicating the solutions that the researcher and supervisor had in mind. Students and course coordinators will also have the chance to ask for a copy of the PhD thesis, once completed - using the email address of the researcher.

(n)Reporting and publication of results. Please indicate which of the following are appropriate. The proposed form of publications should be indicated on the information sheet and/or consent form.

(i) publication in academic or professional journals

(ii) dissemination at academic or professional conferences

Y

(iii) deposit of the research paper or thesis in the University Library (student research)

(iv) a case study used for teaching purposes $\quad \mathbf{N}$

(v) other (please specify) 
Signature of investigators as listed on page 1 (including supervisors) and Chair of SIM HEC.

NB: All investigators and the Chair of SIM HEC must sign the form, then send it to the SIM HEC administrator for filing once the electronic application has been approved.

Date.

Date.

Date.

Supervisor:

Flavio Ferrarotti

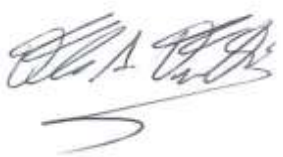

Date: 05/12/2011

Chair of SIM HEC:

Date 


\section{SIM HUMAN ETHICS COMMITTEE \\ Comments on Application for Human Ethics Approval}

Date: 1 December 2011

Principal Researcher: Van Tran Bao Le

Research Project: Discovering semantically meaningful constraints from sample

data - Foundations, algorithms and evaluation

Supervisor: A.Prof. Sebastian Link

Reference No: \#18986

Your HEC application has been reviewed and the Committee's decision is the following:

Approval Given after Amendments made - Application accepted.

Human Ethics Approval valid until: (Date: as in application or no more than 3 years)

Thank you for the amendments you have made to your HEC application. These meet the committee's required changes. On behalf of the HEC Chair I am authorised to inform you that you may now proceed with your research. You may begin your data collection immediately but please note that a hard copy of your application signed by both you and your supervisor (or other researchers involved for staff applications) is required within one month to ensure that we have a complete record of the approval of your application. This should be submitted to me at:

Wendy Chen

School of Information Management

Victoria University of Wellington

Room 518, Rutherford House

23 Lambton Quay

Wellington

Additional Comments (dealing with non-ethical issues only)

Feedback to participants would normally be in the form of a summary of the research outcomes. They would probably find it easier to digest than the entire thesis!

SIM Human Ethics Committee 


\section{Appendix B}

\section{Experiment Information and Consent Forms}

The following documents are information and consent forms for participants to read and consent to participate in our experiments. 
VICTORIA UNIVERSITY OF WELLINGTON

Te Whare Wananga o te Upoko o te Ika a Maui

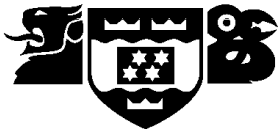

\section{INFORMATION SHEET}

\section{Project Title: Discovering semantically meaningful constraints from sample data -Foundations, algorithms and evaluation}

This research is being conducted by Van Tran Bao Le as part of a PhD thesis, under the supervision of Associate Professor Sebastian Link from the Department of Computer Science at the University of Auckland, and Doctor Flavio Ferrarotti of the School of Information Management, Victoria University, Wellington, New Zealand. The objective of this research is to investigate the usefulness of sample databases, formally called Armstrong databases, to database designers as a means to identify database constraints that are meaningful to the underlying application domain.

If you agree to participate in this research, you will act as a member of a database design team. For a given application domain, the task of the team will be to identify all meaningful functional dependencies and uniqueness constraints, i.e., all those constraints that you expect every future database instance to satisfy. The researcher and her supervisors will act as domain experts in the process of identifying the constraints. That is, they will be happy to answer any questions you might have about the application domain in English language. Since they act in the role of domain experts, they do not have any knowledge about the formal specification of functional dependencies and uniqueness constraints. This is your task. After your team has come up with some agreement about the functional dependencies and uniqueness constraints, please write these down on the sheet allocated to your team, and hand it to the researcher or her supervisors.

The researcher or her supervisors will then use a toolbox to compute a specific sample database (a so-called Armstrong database) that is a concise representation of the constraints you have specified. If you have perceived a functional dependency or uniqueness constraint as meaningful, then the sample database will satisfy it. If you have perceived such a constraint as meaningless, then the sample database will violate it. The sample database will be returned to you. As a team, you have then the opportunity to revise your set of constraints based on the sample database provided. You may observe by looking at the sample that some of the constraints you have specified previously, do not make sense after all, and therefore remove them. 
You may also observe that some of the patterns in the sample do not make sense, because you missed to specify some constraint. In that case, you can add this constraint to your revised set. After you have come to an agreement in your team, please write down your revised constraint set. Time permitting; there might be another round with a new sample database that represents your revised constraint set. At the end, you will be shown the target constraint set that we had in mind for the application domain.

The experiment will be conducted during a tutorial slot for the database course you are currently enrolled in. Your participation in this project is completely voluntary, and you will neither be assessed nor judged according to the input you provide. You may choose to withdraw from your participation at any point during the experiment without having to give reasons. In this case, you may also choose that information provided by you will not be used. Your decision to participate, or refuse to participate in the experiment or to withdraw during the experiment will not, in any way, affect your course results. The only data that is of interest to this research consists of the several sets of functional dependencies and uniqueness constraints that your team decides to specify. The origin of this data will not be recorded. These points have been acknowledged by your course co-ordinator in writing, please see the attached document.

The findings of this research will be reported in a PhD thesis, a conference paper, or a journal article. Raw data will be reported in aggregated form in such a way that individual persons or organisations are not identifiable. All written material will be kept in a locked file and access will be restricted to the investigator. All electronic information will be kept in a password-protected file and access will be restricted to the investigator. All notes and materials will be destroyed 2 years after the conclusion of the research.

Approval for this project has been given by the Human Ethics Committee of Victoria University of Wellington.

Should you have any questions about the study or its progress or would like an electronic copy of the final PhD thesis, please feel free to contact:

Van Tran Bao Le

Email:van.t.le@vuw.ac.nz

\begin{tabular}{|l|l|}
\hline AProf. Sebastian Link & Dr. Flavio Ferrarotti \\
School of Information Management & School of Information Management \\
Victoria University of Wellington & Victoria University of Wellington \\
Room 524, Rutherford House & Room 527, Rutherford House \\
23 Lambton Quay, Pipitea Campus & 23 Lambton Quay, Pipitea Campus \\
Phone: 04 463 6813 & Phone: : 04 463 6857 \\
E-mail: Sebastian.Link@vuw.ac.nz & Email:Flavio.Ferrarotti@vuw.ac.nz \\
\hline
\end{tabular}




\section{Project Title: Discovering semantically meaningful constraints from sample data -Foundations, algorithms and evaluation}

I have been given an Information Sheet about this research and understood the explanations given. I have had the opportunity to ask questions and have them answered to my satisfaction.

I understand that the participation of any student in this project is completely voluntary. I also understand that the students may withdraw from their participation during the experiment without having to give reasons. In this case, they may choose that any information they have provided as part of their participation will be excluded from the data collection and analysis.

I understand that the students will not be assessed nor judged for their contribution to this research project. In particular, the students' decision to participate, or refuse to participate in the experiment or to withdraw during the experiment will not, in any way, affect their course results.

$\square \quad$ I understand that the data collected in this research may be used for research reports of a $\mathrm{PhD}$ thesis, a conference paper, or a journal article. I understand that the raw data will be reported in aggregated form in such a way that individual persons or organisations are not identifiable. All written material will be kept in a locked file and access will be restricted to the investigator. All electronic information will be kept in a password-protected file and access will be restricted to the investigator. All notes and materials will be destroyed two years after the conclusion of the research.

$\square$ I give consent that the researcher and his supervisor can utilize tutorial time of my course to conduct their experiment.

Signed:

Date:

Name:

Co-ordinator of: 
VICTORIA UNIVERSITY OF WELLINGTON

Te Whare Wananga o te Upoko o te Ika a Maui

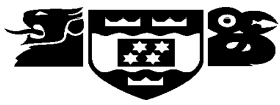

\section{RESEARCH PARTICIPANT CONSENT \& SIGN OFF FORM}

\section{Project Title: Discovering semantically meaningful constraints from sample data -Foundations, algorithms and evaluation}

I have been given an Information Sheet about this research and understood the explanations given. I have had the opportunity to ask questions and have them answered to my satisfaction.

I understand that the researcher and his supervisor have written consent from the course co-ordinator to conduct their experiment during this tutorial, and when requested, I have been shown the sign-off form.

I understand that my participation in this project is completely voluntary. I also understand that I may withdraw from my participation during the experiment without having to give reasons. In this case, I may choose that any information I have provided as part of my participation will be excluded from the data collection and analysis.

I understand that I will not be assessed nor judged for my contribution to this research project. In particular, I understand that my decision to participate, or refuse to participate in the experiment or to withdraw during the experiment will not, in any way, affect my course results.

I understand that this consent form will be kept securely.

I understand that the data collected in this research may be used for research reports of a $\mathrm{PhD}$ thesis, a conference paper, or a journal article. I understand that the raw data will be reported in aggregated form in such a way that individual persons or organisations are not identifiable. All written material will be kept in a locked file and access will be restricted to the investigator. All electronic information will be kept in a password-protected file and access will be restricted to the investigator. All notes and materials will be destroyed two years after the conclusion of the research.

I give my consent to participate in this study.

Signed:

Date: 
238APPENDIX B. EXPERIMENT INFORMATION AND CONSENT FORMS 


\section{Bibliography}

[1] S. Abiteboul, R. Hull, and V. Vianu. Foundations of Databases. Addison-Wesley, 1995.

[2] M. Albrecht, E. Buchholz, A. Düsterhöft, and B. Thalheim. An informal and efficient approach for obtaining semantic constraints using sample data and natural language processing. In Semantics in Databases, volume 1358 of Lecture Notes in Computer Science, pages 1-28. Springer, 1998.

[3] B. Alexe, P.G. Kolaitis, and W.-C. Tan. Characterizing schema mappings via data examples. In Proceedings to the Twenty-Ninth ACM SIGMOD-SIGACT-SIGART Symposium on Principles of Database Systems (PODS), pages 261-271, 2010.

[4] G. Anthes. Happy Birthday, RDBMS! Communications of the ACM, 53(5):16-17, 2010.

[5] M. Arenas and L. Libkin. A normal form for XML documents. ACM Transactions on Database Systems, 29(1):195-232, 2004.

[6] M. Arenas and L. Libkin. An information-theoretic approach to normal forms for relational and XML data. Journal of the ACM, 52(2):246-283, 2005.

[7] W. W. Armstrong. Dependency structures of database relationships. Information Processing, 74:580-583, 1974. 
[8] P. Atzeni and N.M. Morfuni. Functional dependencies and constraints on null values in database relations. Information and Control, 70(1):1-31, 1986.

[9] J. Baixeries and J.L. Balcázar. Characterization and Armstrong relations for degenerate multivalued dependencies using formal concept analysis. In Proceedings of the Third International Conference on Formal Concept Analysis (ICFCA), volume 3403 of Lecture Notes in Computer Science, pages 162-175. Springer, 2005.

[10] C. Beeri and P.A. Bernstein. Computational problems related to the design of normal form relational schemas. ACM Transactions on Database Systems, 4(1):30-59, 1979.

[11] C. Beeri, M. Dowd, R. Fagin, and R. Statman. On the structure of Armstrong relations for functional dependencies. Journal of the ACM, 31(1):30-46, 1984.

[12] P. A. Bernstein. Synthesizing third normal form relations from functional dependencies. ACM Transactions on Database Systems, 1(4):277-298, 1976.

[13] J. Bisbal and J. Grimson. Database sampling with functional dependencies. Information $\mathcal{E}$ Software Technology, 43(10):607-615, 2001.

[14] J. Biskup, D.W. Embley, and J.-H. Lochner. Reducing inference control to access control for normalized database schemas. Information Processing Letters, 106(1):8-12, 2008.

[15] D. Bitton, J.C. Millman, and S. Torgersen. DBE: An expert tool for database design. In Proceedings of the Third International Conference on Advanced Information Systems Engineering (CAiSE), volume 498 of Lecture Notes in Computer Science, pages 240-263. Springer, 1991.

[16] B.W. Boehm. Software Engineering Economics. Prentice Hall, 1981. 
[17] M. Bojanczyk, A. Muscholl, T. Schwentick, and L. Segoufin. Twovariable logic on data trees and XML reasoning. Journal of the ACM, 56(3), 2009.

[18] E. Börger, E. Grädel, and Y. Gurevich. The Classical Decision Problem. Perspectives in Mathematical Logic. Springer, 1997.

[19] A. Brodsky, C. Farkas, and S. Jajodia. Secure databases: Constraints, inference channels, and monitoring disclosures. IEEE Transactions on Knowledge and Data Engineering, 12(6):900-919, 2000.

[20] P. Buneman, S.B. Davidson, W. Fan, C.S. Hara, and W.C. Tan. Reasoning about keys for XML. Information Systems, 28(8):1037-1063, 2003.

[21] P. Buneman, W. Fan, J. Siméon, and S. Weinstein. Constraints for semi-structured data and XML. SIGMOD Record, 30(1):47-54, 2001.

[22] CA Technologies. ERwin Data Modeler - methods guide. https://support.ca.com/cadocs/0/e002961e.pdf, page 86, 2011.

[23] A. Cali, D. Calvanese, G.D. Giacomo, and M. Lenzerini. Data integration under integrity constraints. Information Systems, 29(2):147163, 2004.

[24] D. Calvanese, G.D. Giacomo, and M. Lenzerini. Identification constraints and functional dependencies in description logics. In Proceedings of the Seventeenth International Joint Conference on Artificial Intelligence (IJCAI), pages 155-160. Morgan Kaufmann, 2001.

[25] M.A. Casanova, R. Fagin, and C.H. Papadimitriou. Inclusion dependencies and their interaction with functional dependencies. Journal of Computer and System Sciences, 28(1):29-59, 1984. 
[26] D. Chamberlin and R. Boyce. SEQUEL: A structured english query language. In Proceedings of the ACM-SIGMOD Workshop, volume 1, pages 249-264. ACM, 1974.

[27] Q. Cheng, J. Gryz, F. Koo, C. Leung, L. Liu, X. Qian, and B. Schiefer. Implementation of two semantic query optimization techniques in DB2 universal database. In Proceedings of the Twenty-Fifth International Conference on Very Large Data Bases (VLDB), pages 687-698. Kaufmann,M., 1999.

[28] E. F. Codd. A relational model of data for large shared data banks. Communications of the ACM, 13(6):377-387, 1970.

[29] E. F. Codd. Understanding relations. ACM SIGFIDET FDT Bulletin, 7(3-4):23-28, 1975.

[30] E. F. Codd. Extending the database relational model to capture more meaning. ACM Transactions on Database Systems, 4(4):397-434, 1979.

[31] E. F. Codd. Missing information (applicable and inapplicable) in relational databases. SIGMOD Record, 15(4):53-78, 1986.

[32] J.W. Creswell. Research design: Qualitative, quantitative, and mixed methods approaches. Sage, London, 2003.

[33] C.J. Date. SQL and relational theory. O'Reilly Media, 2 edition, 2011.

[34] C.J. Date and H. Darwen. A Guide to SQL Standard, 4th Edition. Addison-Wesley, 1997.

[35] F. De Marchi, S. Lopes, J.-M. Petit, and F. Toumani. Analysis of existing databases at the logical level: the DBA companion project. SIGMOD Record, 32(1):47-52, 2003.

[36] F. De Marchi and J.-M. Petit. Semantic sampling of existing databases through informative Armstrong databases. Information Systems, 32(3):446-457, 2007. 
[37] J. Demetrovics. On the number of candidate keys. Information Processing Letters, 7(6):266-269, 1978.

[38] J. Demetrovics, G.O.H. Katona, D. Miklós, O. Seleznjev, and B. Thalheim. Asymptotic properties of keys and functional dependencies in random databases. Theoretical Computer Science, 190(2):151-166, 1998.

[39] A. Deutsch, B. Ludäscher, and A. Nash. Rewriting queries using views with access patterns under integrity constraints. Theoretical Computer Science, 371(3):200-226, 2007.

[40] J. Diederich and J. Milton. New methods and fast algorithms for database normalization. ACM Transactions on Database Systems, 13(3):339-365, 1988.

[41] R. Elmasri and S.B. Navathe. Fundamentals of Database Systems. Addison-Wesley, 6 edition, 2010.

[42] A. Enders and H.D. Romback. A Handbook of Software and Systems Engineering; Empirical Overservations, Laws and Theories. AddisonWesley, 2003.

[43] B. Evjen, S. Hanselman, and D. Rader. Professional ASP.NET 4 in C\# and VB. Wrox Press Ltd., Birmingham, UK, UK, 2010.

[44] B. Evjen, S. Hanselman, D. Rader, C. Nagel, J. Glynn, K. Watson, M. Skinner, S. Klein, and T. McCarthy. .NET 3.5 Wrox Box: Professional ASP.NET 3.5, Professional C\# 2008, Professional LINQ, .NET Domain-Driven Design with C\#. Wiley, 2008.

[45] R. Fagin. Functional dependencies in a relational data base and propositional logic. IBM Journal of Research and Development, 21(6):543-544, 1977. 
[46] R. Fagin. Multivalued dependencies and a new normal form for relational databases. ACM Transactions on Database Systems, 2(3):262$278,1977$.

[47] R. Fagin. Armstrong databases. Technical Report RJ3440(40926), IBM Research Laboratory, San Jose, California, USA, 1982.

[48] R. Fagin. Horn clauses and database dependencies. Journal of the ACM, 29(4):952-985, 1982.

[49] R. Fagin and M. Vardi. The theory of data dependencies - a survey. In Mathematics of Information Processing, pages 19-72. American Mathematical Society, 1986.

[50] R. Fagin and M.Y. Vardi. Armstrong databases for functional and inclusion dependencies. Information Processing Letters, 16(1):13-19, 1983.

[51] W. Fan, F. Geerts, and X. Jia. A revival of integrity constraints for data cleaning. Proceedings of the VLDB Environment, 1(2):1522-1523, 2008.

[52] W. Fan, F. Geerts, and X. Jia. Semandaq: a data quality system based on conditional functional dependencies. Proceedings of the VLDB Endowment, 1(2):1460-1463, 2008.

[53] W. Fan, S. Ma, Y. Hu, J. Liu, and Y. Wu. Propagating functional dependencies with conditions. Proceedings of the VLDB Endowment, 1(1):391-407, 2008.

[54] W. Feng. A generalization of boundary value analysis for input parameters with functional dependency. In Proceedings of the Ninth IEEE/ACIS International Conference on Computer and Information Science (ICIS), pages 776-781, 2010. 
[55] F. Ferrarotti, S. Hartmann, H. Köhler, and S. Link. Foundations for a fourth normal form over SQL-like databases. In Conceptual Modelling and Its Theoretical Foundations, volume 7260 of Lecture Notes in Computer Science, pages 85-100. Springer, 2012.

[56] F. Ferrarotti, S. Hartmann, V.T.B. Le, and S. Link. Codd table representations under weak possible world semantics. In Proceedings of the Twenty-Second International Conference on Database and Expert Systems Applications (DEXA), Part I, volume 6860 of Lecture Notes in Computer Science, pages 125-139. Springer, 2011.

[57] R. Garcia. Advanced SQL Server 2005-2008 for the Experienced Professional. Lulu Enterprises Incorporated, 2008.

[58] D. Geiger and J. Pearl. Logical and algorithmic properties of conditional independence and graphical models. The Annals of Statistics, 21(4):2001-2021, 1993.

[59] G. Gottlob, R. Pichler, and F. Wei. Tractable database design through bounded treewidth. Information Systems, 35(3):278-298, 2010.

[60] G. Gottlob and R. Zicari. Closed world databases opened through null values. In Proceedings of the Fourteenth International Conference on Very Large Data Bases (VLDB), pages 50-61. Morgan Kaufmann, 1988.

[61] G. Grahne. Dependency satisfaction in databases with incomplete information. In Proceedings of the Tenth International Conference on Very Large Data Bases (VLDB), pages 37-45. Morgan Kaufmann, 1984.

[62] J. Grant. Null values in a relational data base. Information Processing Letters, 6(5):156-157, 1977.

[63] S. Hartmann. On the implication problem for cardinality constraints and functional dependencies. Annals of Mathematics and Artificial Intelligence, 33(2-4):253-307, 2001. 
[64] S. Hartmann, M. Kirchberg, and S. Link. Design by example for SQL table definitions with functional dependencies. The VLDB Journal, 21(1):121-144, 2012.

[65] S. Hartmann, U. Leck, and S. Link. On Codd families of keys over incomplete relations. The Computer Journal, 54(7):1166-1180, 2011.

[66] S. Hartmann and S. Link. Characterising nested database dependencies by fragments of propositional logic. Annals of Pure and Applied Logic, 152(1):84-106, 2008.

[67] S. Hartmann and S. Link. Efficient reasoning about a robust XML key fragment. ACM Transactions on Database Systems, 34(2), 2009.

[68] S. Hartmann and S. Link. Numerical constraints on XML data. Information and Computation, 208(5):521-544, 2010.

[69] S. Hartmann and S. Link. When data dependencies over SQL tables meet the Logics of Paradox and S-3. In Proceedings to the TwentyNinth ACM SIGMOD-SIGACT-SIGART Symposium on Principles of Database Systems (PODS), pages 317-326, 2010.

[70] S. Hartmann and S. Link. The implication problem of data dependencies over SQL table definitions: Axiomatic, algorithmic and logical characterizations. ACM Transactions on Database Systems, 37(2):13, 2012.

[71] T. Imielinski. Incomplete information in logical databases. IEEE Data Engineering Bulletin, 12(2):29-40, 1989.

[72] T. Imielinski and W. J. Lipski. Incomplete information in relational databases. Journal of the ACM, 31(4):761-791, 1984.

[73] T. Imielinski, V.D. R. Meyden, and K.V. Vadaparty. Complexity tailored design: a new design methodology for databases with incom- 
plete information. Journal of Computer and System Sciences, 51(3):405432, 1995.

[74] A. Klug and R. Price. Determining view dependencies using tableaux. ACM Transactions on Database Systems, 7(3):361-380, 1982.

[75] H. Koehler, U. Leck, and S. Link. Possible and certain SQL keys. Technical Report CDMTCS 452, Centre for Discrete Mathematics and Theoretical Computer Science, The University of Auckland, New Zealand, 2013.

[76] S. Kolahi. Dependency-preserving normalization of relational and XML data. Journal of Computer and System Sciences, 73(4):636-647, 2007.

[77] W.-D. Langeveldt and S. Link. Empirical evidence for the usefulness of Armstrong relations in the acquisition of meaningful functional dependencies. Information Systems, 35(3):352-374, 2010.

[78] S. Lauesen and O. Vinter. Preventing requirement defects: An experiment in process improvement. Requirements Engingeering, 6(1):3750, 2001.

[79] G. Lausen. Relational databases in RDF: Keys and foreign keys. In Proceedings of the VLDB Workshop on Semantic Web, Ontologies and Databases (SWDB-ODBIS), volume 5005 of Lecture Notes in Computer Science, pages 43-56. Springer, 2007.

[80] G. Lausen, M. Meier, and M. Schmidt. SPARQLing constraints for RDF. In Proceedings of the Eleventh International Conference on Extending Database Technology (EDBT), volume 261 of ACM International Conference Proceeding Series, pages 499-509, 2008.

[81] V.T.B. Le, S. Link, and F. Ferrarotti. Effective recognition and visualization of semantic requirements by perfect SQL samples. In Proceed- 
ings of the Thirty-Second International Conference on Conceptual Modeling (ER), volume 8217 of Lecture Notes in Computer Science, pages 227-240. Springer, 2013.

[82] V.T.B. Le, S. Link, and F. Ferrarotti. SQL-sampler: A tool to visualize and consolidate domain semantics by perfect SQL sample data. In Proceedings of the Tenth Asia-Pacific Conference on Conceptual Modelling (APCCM), volume 154 of Conferences in Research and Practice in Information Technology, page 10 pages. Australian Computer Society, 2014.

[83] V.T.B. Le, S. Link, and M. Memari. Discovery of keys from SQL tables. In Proceedings of the Seventeenth International Conference on Database Systems for Advanced Applications (DASFAA), volume 7238 of Lecture Notes in Computer Science, pages 48-62. Springer, 2012.

[84] V.T.B. Le, S. Link, and M. Memari. Schema- and data-driven discovery of SQL keys. Journal of Computing Science and Engineering, 6(3):193-206, 2012.

[85] P.D. Leedy and J.E. Ormrod. Pratical Research - Planning and Desgin. Merill-Prentice Hall, New Jersy, 2001.

[86] M. Levene and G. Loizou. Axiomatisation of functional dependencies in incomplete relations. Theoretical Computer Science, 206(12):283-300, 1998.

[87] M. Levene and G. Loizou. A Guided Tour of Relational Databases and Beyond. Springer, 1999.

[88] L. Libkin and L. Wong. Semantic representations and query languages for Or-sets. Journal of Computer and System Sciences, 52(1):125$142,1996$.

[89] E.Y. Lien. On the equivalence of database models. Journal of the ACM, 29(2):333-362, 1982. 
[90] T.W. Ling. A normal form for entity-relationship diagrams. In Proceedings of the Fourth International Conference on Entity-Relationship Approach, pages 24-35. IEEE Computer Society, 1985.

[91] S. Link. Armstrong databases: Validation, communication and consolidation of conceptual models with perfect test data. In Proceedings of the Eighth Asia-Pacic Conference on Conceptual Modelling (APCCM), volume 130 of Conferences in Research and Practice in Information Technology, pages 3-20. Australian Computer Society, 2012.

[92] S. Link. Reasoning about domain semantics over relations, bags, partial relations and partial bags. In Proceedings of the Fourth International Conference on Advances in Databases, Knowledge, and Data Applications (DBKDA), page 8 pages. Thinkmind, 2012.

[93] S. Link. Sound approximate reasoning about saturated conditional probabilistic independence under controlled uncertainty. Journal of Applied Logic, 11(3):309-327, 2013.

[94] D. Maier. The Theory of Relational Databases. Pitman Publishing Limited, 1983.

[95] A. Makinouchi. A consideration on normal form of not-necessarilynormalised relation in the relational data model. In Proceedings of the Third International Conference on Very Large Data Bases (VLDB), pages 447-453. Morgan Kaufmann, 1977.

[96] H. Mannila and K.-J. Räihä. Design by example: An application of Armstrong relations. Journal of Computer and System Sciences, 33(2):126-141, 1986.

[97] H. Mannila and K.-J. Räihä. Design of Relational Databases. AddisonWesley, 1992. 
[98] B. Marnette, G. Mecca, and P. Papotti. Scalable data exchange with functional dependencies. Proceedings of the VLDB Endowment, 3(1):105-116, 2010.

[99] J. Martin. Information Engineering. Prentice Hall, 1989.

[100] E. Meijer and G. Bierman. A co-relational model of data for large shared data banks. Communications of the ACM, 54(4):49-58, 2011.

[101] R. J. Miller, M. A. Hernandez, L. M. Haas, L.-L. Yan, C. T. H. Ho, R. Fagin, and L. Popa. The Clio project: managing heterogeneity. SIGMOD Record, 30(1):78-83, 2001.

[102] J.C. Mitchell. The implication problem for functional and inclusion dependencies. Information and Control, 56(3):154-173, 1983.

[103] J. Paredaens. What about constraints in RDF? In Conceptual Modelling and Its Theoretical Foundations, volume 7260 of Lecture Notes in Computer Science, pages 7-18. Springer, 2012.

[104] G. N. Paulley. Exploiting functional dependence in query optimization. PhD thesis, University of Waterloo, 2001.

[105] L. Qian, K. LeFevre, and H.V. Jagadish. CRIUS: User-friendly database design. Proceedings of the VLDB Endowment, 4(2):81-92, 2011.

[106] O. Rauh and E. Stickel. Standard transformations for the normalization of ER schemata. In Proceedings of the Seventh International Conference on Advanced Information Systems Engineering (CAiSE), volume 932 of Lecture Notes in Computer Science, pages 313-326. Springer, 1995.

[107] A. Rosenthal and D. Reiner. Tools and transformationsrigorous and otherwisefor practical database design. ACM Transactions on Database Systems, 19(2):167-211, 1994. 
[108] M. Rys. Scalable SQL. ACM Queue, 9(4):8 pages, 2011.

[109] A.D. Sarma, J.D. Ullman, and J. Widom. Schema design for uncertain databases. In Proceedings of the Third Alberto Mendelzon International Workshop on Foundations of Data Management, volume 450 of CEUR Workshop Proceedings, 2009.

[110] K. Schaefer, J. Cochran, S. Forsyth, R. Baugh, M. Everest, and D. Glendenning. Professional IIS 7. Wrox Press Ltd., Birmingham, UK, UK, 2008.

[111] M. Schaerf and M. Cadoli. Tractable reasoning via approximation. Artificial Intelligence, 74:249-310, 1995.

[112] A.M. Silva and M.A. Melkanoff. A method for helping discover the dependencies of a relation. In Advances in Data Base Theory, pages 115-133, 1979.

[113] M.I. Sözat and A. Yazici. A complete axiomatization for fuzzy functional and multivalued dependencies in fuzzy database relations. ACM Fuzzy Sets and Systems, 117(2):161-181, 2001.

[114] Standish Group. The CHAOS report. The Standish Group International, available on-line at http://www.standishgroup.com/sample_research/ chaos_1994_1.php, 1994.

[115] Standish Group. Unfinished voyages. The Standish Group International, available on-line at http://www.standishgroup.com/sample_research/ unfinsihed_voyages_1.php, 1995.

[116] S. Staworko and J. Chomicki. Consistent query answers in the presence of universal constraints. Information Systems, 35(1):1-22, 2010. 
[117] D. Suciu, D. Olteanu, C. Ré, and C. Koch. Probabilistic Databases. Synthesis Lectures on Data Management. Morgan \& Claypool Publishers, 2011.

[118] H.B.K. Tan and Y. Zhao. Automated elicitation of functional dependencies from source codes of database transactions. Information and Software Technology, 46(2):109-117, 2004.

[119] Z. Tari, J. Stokes, and S. Spaccapietra. Object normal forms and dependency constraints for object-oriented schemata. ACM Transactions on Database Systems, 22(4):513-569, 1997.

[120] B. Thalheim. On the number of keys in relational databases. In Proceedings of the Sixth International Conference on Fundamentals of Computation Theory (FCT), volume 278 of Lecture Notes in Computer Science, pages 448-455. Springer, 1987.

[121] B. Thalheim. On semantic issues connected with keys in relational databases permitting null values. Elektronische Informationsverarbeitung und Kybernetik, 25(1/2):11-20, 1989.

[122] B. Thalheim. Dependencies in relational databases. Teubner, 1991.

[123] B. Thalheim. Entity-Relationship modeling: Fundamentals of database technology. Springer, Heidelberg, Germany, 2000.

[124] D. Toman and G. Weddell. On keys and functional dependencies as first-class citizens in description logics. Journal of Automated Reasoning, 40(2):117-132, 2008.

[125] J.D. Ullman. Principles of Database and Knowledge-base System. Computer Science Press, Rockville, 1988.

[126] K.V. Vadaparty and S.A. Naqvi. Using constraints for efficient query processing in nondeterministic databases. IEEE Transactions on Knowledge and Data Engineering, 7(6):850-864, 1995. 
[127] V. Vianu. A web odyssey: from Codd to XML. SIGMOD Record, 32(2):68-77, 2003.

[128] M.W. Vincent. Semantic foundations of $4 \mathrm{NF}$ in relational database design. Acta Informatica, 36(3):173-213, 1999.

[129] M.W. Vincent, J. Liu, and C. Liu. Strong functional dependencies and their application to normal forms in XML. ACM Transactions on Database Systems, 29(3):445-462, 2004.

[130] M.W. Vincent, J. Liu, and M.K Mohania. The implication problem for 'closest node' functional dependencies in complete XML documents. Journal of Computer and System Sciences, 78(4):1045-1098, 2012.

[131] D.Z. Wang, X.L. Dong, A.D. Sarma, M.J. Franklin, and A.Y. Halevy. Functional dependency generation and applications in pay-as-yougo data integration systems. In Proceedings of the Twelfth International Workshop on the Web and Databases (WebDB), 2009.

[132] H. Wang and R. Liu. Privacy-preserving publishing microdata with full functional dependencies. Data \& Knowledge Engineering, 70:249268, 2011.

[133] G. E. Weddell. Reasoning about functional dependencies generalized for semantic data models. ACM Transactions on Database Systems, 17(1):32-64, 1992.

[134] C. Zaniolo. Database relations with null values. Journal of Computer and System Sciences, 28(1):142-166, 1984.

[135] W. Ziarko. The discovery, analysis, and representation of data dependencies in databases. In Knowledge Discovery in Databases, pages 195-212. MIT Press, 1991. 
[136] R.E. Zultner. The deming way: Total quality management for software. In Proceedings of Total Quality Management for Software, pages 134-145, 1992. 